\title{
Development and Evaluation of Novel, Large Area, Radiation Hard Silicon Microstrip Sensors for the ATLAS ITk Experiment at the HL-LHC
}

\author{
by \\ Robert Francis Holub Hunter, B.Sc. \\ A thesis submitted to the \\ Faculty of Graduate and Postdoctoral Affairs \\ in partial fulfillment of the requirements for the degree of
}

Master of Science in Experimental Particle Physics

Ottawa-Carleton Institute for Physics

Department of Physics

Carleton University

Ottawa, Ontario

August, 2017

(C) Copyright

Robert Francis Holub Hunter, 2017 
The undersigned hereby recommends to the

Faculty of Graduate and Postdoctoral Affairs acceptance of the thesis

\title{
Development and Evaluation of Novel, Large Area, Radiation Hard Silicon Microstrip Sensors for the ATLAS ITk Experiment at the HL-LHC
}

\author{
submitted by Robert Francis Holub Hunter, B.Sc. \\ in partial fulfillment of the requirements for the degree of \\ Master of Science in Experimental Particle Physics
}

Professor Thomas Koffas, Thesis Supervisor

Ottawa-Carleton Institute for Physics

Department of Physics

Carleton University

August, 2017 


\section{Abstract}

Development and evaluation of novel, large area, radiation hard silicon microstrip sensors is necessary for the operation of the ATLAS detector at the high luminosity upgrade of the LHC particle accelerator.

This next-generation collider will allow an order of magnitude more premier physics data to be obtained from the facility that produces the world-leading 13 - $14 \mathrm{TeV}$ centre-of-mass frame collisional energy. The physics impact of this coupled nature is fundamental as the sensitivity to any interaction that falls within this massive energy range is naturally increased by the reduction in statistical uncertainties. This poses two prime opportunities for the discovery of new physics; precision measurement of known interactions and sensitive uncovering of the unknown.

The HL-LHC's increases in collision rate and experiment lifetime necessitate complimentary changes to the LHC detectors' layouts, granularities, readouts, and radiation hardness. For the general purpose ATLAS detector the innermost sub-component must be entirely replaced to be able to operate in the HL-LHC environment. The ITk detector is the all-silicon, radiation resistant replacement.

The ITk Strip detector which occupies the outer portion of the ITk volume will be most sensitive to the combination of silicon surface and radiation damage increase. For it to be realized it must use unique large area, radiation hard sensors. The development and evaluation of these are the subject of this manuscript. 
To Carolina, Mom, and Dad. For your unbounded support. 


\section{Acknowledgments}

I would like to thank all of the large number of people who made this work possible. Foremost among these is my supervisor, Dr. Thomas Koffas, whose tireless, insightful guidance was integral to my, and this document's, development.

I would also like to express special thanks to the other members of the Carleton ITk research group; Dr. Dag Gillberg, James Botte, Dr. John Keller, Steven Lee, and Matt Park, for their support and camaraderie. To the Carleton Physics and Electronics Departments' technical staff; Rob Vandeusen, Philippe Gravelle, and Matt Bowcock, for their irreplaceable help with setting up the Carleton University sensor test clean-room. And to the Carleton ATLAS group and Physics Department for the opportunities, experiences, and knowledge that have led me this point in my research career.

I would also like to extend a special thanks to Dr. Bart Hommels and Christoph Klein for the gracious and informative tour of their lab. To Drs. Susanne Keuhn and Andrew Blue from my testbeam experiences for their multi-threaded guidance. And to the AIDA-2020 organization for partial funding thereof.

Finally, my sincerest thanks go to my parents and my partner, Carolina Eulert, who have stood by me with encouraging reassurance through the difficult periods of life and the writing of this sizable manuscript. To Steve and my extended family for they're patience and inspiration. And to Scotch and Harley for their calming influence. 


\section{Table of Contents}

Abstract $\quad$ iii

Acknowledgments $\quad$ v

Table of Contents vi vi v

List of Tables $\quad$ x

List of Figures $\quad$ xii

1 The Inner Tracker Upgrade 1

1.1 HL-LHC . . . . . . . . . . . . . . . . . . . 2

1.1.1 The Standard Model and Its Experimental Handles . . . . . . 3

1.1.2 The LHC and Its Importance . . . . . . . . . . . . . . . . 4

1.1.3 The HL-LHC and Its Importance . . . . . . . . . . . . . . 6

1.1.4 HL-LHC Upgrade Schedules . . . . . . . . . . . . . . . 8

1.2 The ATLAS Detector . . . . . . . . . . . . . . . . . . 10

1.2.1 Overview ......................... 11

1.2.2 ATLAS Upgrade Schedule . . . . . . . . . . . . . . . . 12

1.3 The Inner Detector and Its Replacement . . . . . . . . . . . . . . . . 13

1.3.1 Structure of the Inner Detector . . . . . . . . . . . . . . . . 14

1.3.2 Deficiencies in the Inner Detector . . . . . . . . . . . . . . 15

1.3.3 General Requirements for the ID Replacement . . . . . . . . . 17

1.4 The Inner Tracker . . . . . . . . . . . . . . . . . . . . . . . . . . . 20

1.4.1 ITk Overview . . . . . . . . . . . . . . . . . . 20

1.4 .2 ITk Layout . . . . . . . . . . . . . . . . . . . . . . . 22

1.4.3 Radiation Environment and Hardness in the ITk . . . . . . . . 23

1.5 Expected Performance of the ITk . . . . . . . . . . . . . . . . 28 
1.5.1 Tracking Requirements . . . . . . . . . . . . . . . 28

1.5.2 Tracking Performance . . . . . . . . . . . . . . . . . . 29

1.5.3 Higgs Physics Benchmark . . . . . . . . . . . . . . . . 34

2 The ITk Strip Detector $\quad 39$

2.1 Detector Overview . . . . . . . . . . . . . . . . . 40

2.2 Detector Architecture . . . . . . . . . . . . . . . . . . . . 41

2.3 ITk Strip Tracking Functionality . . . . . . . . . . . . . 45

2.4 The ITk Strip Module . . . . . . . . . . . . . . . . . . . . . . . 48

2.5 Overall Electrical Architecture . . . . . . . . . . . . . . . . 53

2.6 ITk Strip Detector Production Model . . . . . . . . . . . . . . . 55

2.6.1 Canada's Role in ITk Production . . . . . . . . . . . . 56

2.7 Prototype Results . . . . . . . . . . . . . . . . . . . . . . . . . . . . . . 59

2.7.1 ABCStar Signal Processing . . . . . . . . . . . . 60

2.7.2 The TID Bump and the ABCStar . . . . . . . . . . . 61

2.7.3 The 2016 Testbeam Campaign and End-of-Lifetime Performance 63

3 The Physics of Silicon Microstrip Detectors 69

3.1 Semiconductors and the P-N Junction . . . . . . . . . 70

3.2 Microstrip Semiconductor Detector; Principle of Operation . . . . . . 74

3.3 Characteristics of Microstrip Semiconductor Detectors _. . . . . . 77

3.3.1 Depletion Capacitance . . . . . . . . . . . . . . . . . 77

3.3.2 Full Depletion Voltage . . . . . . . . . . . . . . . 78

3.3.3 Charge Collection Efficiency . . . . . . . . . . . . . 80

3.3.4 Leakage Current . . . . . . . . . . . . . . . . . . . . 81

3.3.5 Junction Breakdown . . . . . . . . . . . . . . . . . . 82

3.3.6 Surface Physics . . . . . . . . . . . . . . . . . . 83

3.3 .7 Noise . . . . . . . . . . . . . . . . . . . . . . . . . 92

3.4 Radiation Damage . . . . . . . . . . . . . . . . . . . . . 92

3.4 .1 Bulk Damage . . . . . . . . . . . . . . . . . . . . . . . 93

3.4.2 Surface Damage . . . . . . . . . . . . . . . . . . 96

3.4 .3 Annealing . . . . . . . . . . . . . . . . . . . 97 97

4 Sensors of the ITk Strip Detector 102

4.1 Overview and Specifications . . . . . . . . . . . . . . . 102 
4.1.1 Introduction to the ITk Strip Sensor Design . . . . . . . . . . 103

4.1.2 Sensor Geometries . . . . . . . . . . . . . . . 108

4.2 Development of the ITk Strip Sensor Design . . . . . . . . . . . . . . 111

4.2.1 Historical Overview . . . . . . . . . . . . . . . . . 112

4.2.2 Dawning of Radiation Hard Silicon Detectors . . . . . . . . . 113

4.2.3 Genesis of a Large Area Radiation Hard Sensor Design . . . . 114

4.3 Innovations of the ITk Strip Sensor Design . . . . . . . . . . . . . . . 119

4.3.1 Radiation Hardness in the Bulk Material . . . . . . . . . . . . 120

4.3.2 Optimization of Surface Layout . . . . . . . . . . . . . . . . . 125

4.3.3 End-cap Specific Considerations . . . . . . . . . . . . . 135

5 Sensor Testing at Carleton University 141

5.1 ITk Strip Sensor Testing Procedures . . . . . . . . . . . . . . . . . . 142

5.1.1 Sensor Quality Control . . . . . . . . . . . . . . . 142

5.1 .2 Sensor QA . . . . . . . . . . . . . . 149

5.2 Production Site Development . . . . . . . . . . . . . . . 152

5.3 Mechanical Tests and Considerations . . . . . . . . . . . . . 156

5.3 .1 Visual Inspection . . . . . . . . . . . . . . . . . 156

5.3.2 Metrology . . . . . . . . . . . . . . . . . . . 158

5.3.3 Mechanical Considerations . . . . . . . . . . . . . . . 159

5.4 Electrical Test Setups . . . . . . . . . . . . . . . . . . . . . 161

5.4.1 Probestation . . . . . . . . . . . . . 163

5.4 .2 Custom Jig . . . . . . . . . . . . . . . . . . . 164

5.4.3 Patch Panels . . . . . . . . . . . . . . . . 166

5.4 .4 Instrumentation . . . . . . . . . . . . . . 167

5.4.5 Sensor Test Software . . . . . . . . . . . . . . . . . . 170

5.4.6 Long-Term Testing Considerations . . . . . . . . . . . . . . . 170

5.4.7 Procedure . . . . . . . . . . . . . . . . 171

5.5 Electrical Test Results of ITk Strip Sensors . . . . . . . . . . . . . . . 172

5.5.1 Leakage Current and the IV Curve . . . . . . . . . . . . . . . 172

5.5.2 Full Depletion Voltage and the CV Curve . . . . . . . . . . . 175

5.5.3 Long Term IV Stability and its Humidity Dependency . . . . 182

$\begin{array}{lll}6 & \text { Summary and Conclusions } & 186\end{array}$ 


\section{List of Tables}

1.1 Summary of current and future infrastructure operational parameters important to the ITk upgrade. Trigger rate will be discussed in the text of this Section (1.3.2). Particle fluence, its unit of $1 \mathrm{MeVn}_{\text {eqv }} \mathrm{cm}^{-2}$, and total ionizing dose will be discussed in context to the ITk in Section 1.4.3 and in context to the silicon sensors in Chapters 3, 4, and 5. [1-3] 16

1.2 Maximal fluence and dose expectations at $4500 \mathrm{fb}^{-1}$. [1] . . . . . . 25

1.3 Basic tracking performance targets for the ITk. Track finding efficiencies for the particles with the most significant physics impact. [1] . . .

1.4 Track reconstruction requirements for the two main psuedorapidity regions of the ITk. Requirements shown are for a "reconstructed" track in the simulation. [1] . . . . . . . . . . . . . . . . . 30

2.1 Summary of the number of components to be used in the ITk Strip Detector, broken into barrel and endcap. [1] . . . . . . . . . . .

2.2 Details of the barrel sensor geometry in terms of the four layer structure. Radius is with respect to the beam axis and tilt angle is with respect to the $r \phi$ plane. Each barrel layer is $2.8 \mathrm{~m}$ long centered on $z=0 .[1] \ldots \ldots \ldots \ldots \ldots \ldots \ldots \ldots \ldots \ldots \ldots \ldots \ldots \ldots \ldots$

2.3 Details of the endcap sensor geometries. Parameters are given for each strip segment of the petal. Inner radius are in respect to the beam axis and the listed strip pitch is the length averaged value. [1] . . . . . . . 47

2.4 Summary of the custom ASIC set used on the ITk Strip module. [1] .

2.5 Estimated signal-to-noise ratios for end-of-lifetime performance. All ITk Strip modules are shown. Fluence includes 1.5 safety factor. [1] .

4.1 A summary of the specifications for ITk Strip detector's large area, $n^{+}-i n-p$, AC-coupled microstrip, oxygenated float zone (FZ) silicon detector. [1] . . . . . . . . . . . . . . . . . . . . . . . . 104 
4.2 An overview of the eight ITk Strip sensor geometries and their main characteristics. The minimum and maximum pitch is across all strip rows. [1] . . . . . . . . . . . . . . . . . . . . . . . . . . . . . . . . . 109

4.3 Dopant levels of the strip isolation structures tested in the A05 to A07 sensors. [4] . . . . . . . . . . . . . . . . . . . . . . . 128 


\section{List of Figures}

1.1 Schematic of CERN's LHC starring accelerator complex. Accelerators, beam lines, and (some) detector experiments are shown. The LHC protons are generated from hydrogen in Linac 2. They then pass through the Proton Synchrotron (PS), the booster, and the Super Proton Synchrotron (SPS) before reaching the LHC main ring. Colors denote accelerated particle type. $[5]$. . . . . . . . . . . . . . .

1.2 Importance of statistics (integrated luminosity). Left; Standard Model predictions for the Higgs boson total production cross-section as a function of Higgs mass and interaction energy. Right; Standard Model precictions for the Higgs boson decay channels as a function of Higgs mass; the 'detectable' Higgs boson cross-section (production c.s. times branching ratio). [6] . . . . . . . . . . . . . .

1.3 Baseline program for the LHC including the HL-LHC phase. The green line signifies instantaneous luminosity, while the red signifies the energy. Nominal luminosity is the LHC nominal; $1 \times 10^{34} \mathrm{~cm}^{-2} \mathrm{~s}^{-1}$. LS stands for long shutdown. [1] . . . . . . . . . . . . . .

1.4 Pileup simulations in conditions akin to the LHC and current ID (top) as well as HL-LHC and ITk (bottom). The detector shown in blue is an old layout of the ITk. [7] . . . . . . . . . . . . . . . . . . . . . 10

1.5 Simplified schematic of the LHC's ATLAS detector (its current iteration). The main sub-detector systems are shown. [8] . . . . . . . . . .

1.6 The detector structure of ATLAS' current inner tracking system, the Inner Dector (ID). The three detector sub-systems of silicon based Pixels and SemiConductor Tracker (SCT), and gas based Transition Radiation Tracker (TRT) are shown. The ID is cylindrical like seen in Figure 1.5. [9] . . . . . . . . . . . . . . . . . . . 
1.7 A visualization of the ITk as implemented in its performance evaluation simulation framework. [1] . . . . . . . . . . . . . . . .

1.8 Simplified schematic layout of the ITk detector. Only active components and one quadrant of the symmetric detector is shown. $\mathrm{x}$-axis is along the beam pipe with the origin at the interaction point. y-axis is outwards in radius of the cylindrical ITk. "Inclined" refers to the layout; it is the front-runner of the two remaining designs. [1] . . . . .

1.9 Material reduction summary. Top; radiation length as a function of $\eta$ for the current ATLAS detector (Top Left) and the ITk (Top Right). The contributions from each type of material are stacked. Bottom; fractional radiation length contributions at the ITk Strip module level, for inner barrel (Bottom Left) and outer barrel (Bottom Right) modules (see Section 2.4 for module description). [1] . . . . . . . . . . . .

1.10 Fluence and dose distributions expected in the ITk at $3000 \mathrm{fb}^{-1}$. Top; the $1 \mathrm{MeVn}_{\text {eqv }} \mathrm{cm}^{-2}$ NIEL fluence. Centre; total ionizing dose (TID). Bottom; charged particle fluence per proton-proton collision in the

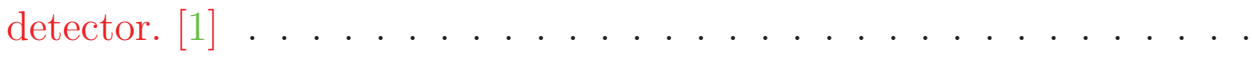

1.11 Charged particle, neutron, and total fluences as a function of radius at $6000 \mathrm{fb}^{-1}$. For the total fluence several beam axis (z) positions are shown (refer Figure 1.8). [10] . . . . . . . . . . . . . .

1.12 Evidence that track reconstruction efficiency demands will be met in the ITk. Simulation results showing predicted hits per track counts as a function of psuedorapidity. Both detector contributions are given. A muon with $10 \mathrm{GeV}$ transverse momentum is simulated, a taxing signature to detect with significant physics impact. [1] . . . . . . . . .

1.13 Occupancy predictions in the ITk and ITk Strip detectors at $3000 \mathrm{fb}^{-1}$ and $\mu=200$. Top; average channel occupancy over the full ITk layout (refer Figure 1.8). Bottom; more detailed views of average channel occupancy for the Strip detector's barrel layers (left) and endcap disks (right). [1] . . . . . . . . . . . . . . . . . . 
1.14 Track reconstruction efficiency projections for the ITk. Left; as a function of psuedorapidity for (mip) muons with 10 or $100 \mathrm{GeV}$ transverse momentum. Run 2 results for the ID are shown in red and orange with the ITk in blue and green. Right; as a function of pileup for the main pseudorapidity regions of the ITk. [1] . . . . . . . . . . . . .

1.15 The "mexican hat" potential (V). The $\phi^{1}$ and $\phi^{2}$ axes correspond to the complex symmetry axes. Since the electroweak symmetry group is $\mathrm{SU}(2)$ this potential defines the minimum energy configuration of the Higgs field. [1] . . . . . . . . . . . . . . . . .

1.16 Example of the reduction in (relative) statistical uncertainty that will be obtained at the LHC. Left; Higgs boson decay widths (refer to Figure 1.2) with the production mechanism contributers shown in brackets. Right; coupling scale factor ratios. A $125 \mathrm{GeV}$ Standard Model Higgs boson is assumed. [12] . . . . . . . . . . . . . . . .

1.17 HL-LHC gains in the $H \rightarrow \mu \mu$ channel. Left; signal (red) overlaid on stacked backgrounds. Right; background subtracted result, a signal significance of seven sigma is obtained. [13] . . . . . . . . . .

2.1 Summary of the major steps in the building of the ITk Strip detector. $[1,14] \ldots \ldots \ldots \ldots \ldots \ldots$

2.2 Summary of the local support structures. Bottom; the barrel stave. Top right; the end-cap petal. Top left; photograph of a thermomechanical petal used for finite analysis verification of thermal models. [1] 44

2.3 Simplified cross-sectional schematic of the stave local support system. The end-cap's petals use the same materials. Not to scale. [1] . . . . .

2.4 Photographs of recent ATLAS ITk prototype modules. Top; a short strip barrel module with ATLAS12 sensor. Bottom; an innermost ring (R0) end-cap prototype module with ATLAS12EC sensor. See Chapter 4 for discussion of sensor types. [1] . . . . . . . . . . . . . .

2.5 A short strip barrel module in exploded view. All major components are shown and are found in both barrel and end-cap modules. [1] . . .

2.6 Technical schematics of the electrical architecture. Top; at the (short strip barrel) module level. Bottom; at the (inner) stave level. Aspects of the schematic are discussed in the accompanying text (Section 2.5). [15] 54 
2.7 Gantt chart representation of the ITk Strip Detector major milestones. Classification in terms of work packages is given. The long-lead time sensors compose the vanguard of the development. [1] . . . . . . . . . 57

2.8 A simplified schematic of the production scheme of the ITk Strip Detector local support structures. Each step is conducted in parallel and detailed aspects of the testing program are not shown. [1] . . . . . . .

2.9 Exemplary hit efficiency vs. binary signal threshold "S-Curve". Data is an ABC130 with an injected calibration charge of $1.51 \mathrm{fC}$. [1] . . .

2.10 "The TID Bump". Digital current of the module versus total ionizing dose for ABC130 front-end chip prototypes. Dose is delivered by Xrays at the rates and temperatures prescribed. [1] . . . . . . . . .

2.11 Signal to noise ratio consequences of TID in the ABC130 and ABC130Star. Left; ABC130 gain as a function of TID. Centre; ABC130 input noise as a function of TID. Right; input noise as a function of TID for the improved radiation hard design of the ABC-

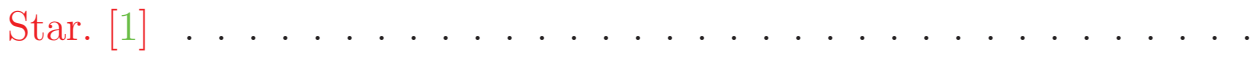

2.12 Photograph of the 2016 testbeam setup at DESY. The six planes of the EUDET telescope are seen on either side of the device under test in its air tight environmental box. The $5 \mathrm{GeV}$ electron beam enters from the right in this photo. . . . . . . . . . . . . . . . . . .

2.13 S-curve results for the irradiated and unirradiated full-size pseudo-long strip modules. Shown is the data for unirradiated LS4 (green) and irradiated LS3 (red) long strip modules. The green curves would show the same tail as the red curve if data had been collected. Results were obtained during the 2016 testbeam campaign. [1] . . . . . . . . . . .

2.14 Proof that signal to noise requirements (dashed lines) will be met, given by early prototypes. Signal efficiency (black data) compared to noise occupancy as a function of readout chip threshold. Noise profiles of the ABC130 (filled red data) and ABCStar (unfilled red data) are given. Top; un-irradiated LS4 prototype long strips at $400 \mathrm{~V}$. Centre row; irradiated LS3 prototype short strips at $500 \mathrm{~V}$ (left) and $600 \mathrm{~V}$ (right). Bottom row; LS3 long strips at 500V (left) and 600 V (right). Test conditions and ATLAS12 prototype sensor quality are below ITk specification (see Section 4.2). [1] . . . . . . . . . . . 
3.1 Band structure variants. Sub-figure (a) shows an insulator or intrinsic semiconductor (dependent upon the size of the band gap). The Fermi energy sits in the band gap. Sub-figures (b) and (c) show a metal with overlapping valence and conduction bands (b) and an unfilled valence band (c). [16] . . . . . . . . . . . . . . . . . . .

3.2 Schematics depicting the band structure, density of states, Fermi-Dirac distribution of energy levels (for a non-zero temperature), and the charge carrier concentrations for (a) intrinsic, (b) n-type, and (c) ptype semiconductors. $[17] \ldots \ldots \ldots \ldots$

3.3 The p-n junction in thermal equilibrium for a junction with an abrupt dopant profile (left) and a gradual dopant profile (right). From top to bottom are the impurity distributions, field distributions, electric potentials, and energy band diagrams across the interface. [17] . . . .

3.4 IV curve of a realistic silicon (dashed line) and ideal (solid line) diode. Current is normalized to the ideal reverse saturation value. Aspects of the reverse bias curve are discussed in the Sections 3.3 and 3.4. [17] .

3.5 Schematic representation of a semiconductor microstrip detector. Shown is a single-sided, $p^{+} i n n$, AC coupled, silicon detector. This design is similar in base operation to the ITk Strip sensor but with the types reversed. [18] . . . . . . . . . . . . . . . . .

3.6 Simulation of electric field strength in a silicon microstrip detector at partial depletion (white line). [19] . . . . . . . . . . . .

3.7 The capacitance-voltage relationship for a microstrip detector biased past the full depletion voltage. The full depletion voltage is easily identifiable from the intersection of the depleting and depleted linear trends. The data shown was collected with the most recent ATLAS ITk Strip prototype (ATLAS12EC) at Carleton University. . . . . .

3.8 The metal-oxide-semiconductor (MOS) structure. At left, a simple schematic of the geometry. At right, the band diagrams at $V=0$ in the cases of $\mathrm{n}$ (top) and $\mathrm{p}$ (bottom). $\chi$ is electron affinity of insulator (i) and semiconductor, $\psi_{B}$ the potential barrier, $\psi_{m}$ the metal work function and $\phi_{B}$ the difference between the Fermi energy and its intrinsic counterpart. [17] . . . . . . . . . . . . . 
3.9 The three regime operation of a MOS under bias. At left, the band diagram representation of the three regimes of accumulation, depletion, and inversion for both $\mathrm{p}$ and $\mathrm{n}$ type semiconductor. At right, the evolution of surface charge, $Q_{S}$ as a function of surface potential, $\psi_{S}$ for a p-type sample with dopant concentration of $4 \times 10^{15} \mathrm{~cm}^{-3} \cdot \psi_{B}$ is the difference between the doped and intrinsic Fermi level. [17] . . . .

3.10 Schematic depiction of the classes of charges and states in an unideal MOS device. Not shown is the charge that accumulates on the outer passivation layer. [17] . . . . . . . . . . . . . . . .

3.11 An example interface between silicon (left) and silicon dioxide (right). The arrows denote "bridge" bonds which are associated with the large nearby vacancy. [20] . . . . . . . . . . . . . . . .

3.12 Measured surface state densities at the silicon - silicon dioxide interface for three silicon crystal orientations. [17] . . . . . . . . . . . . .

3.13 IV characteristic of microdischarge. Sensor leakage current as a function of bias voltage and voltage across the integrated capacitor. (Bias from strip to bias implant.) $[21] \ldots$. . . . . . . . . . .

3.14 An example of the effect geometry plays on field strength at the sensor surface. At left; the onset voltage of microdischarge is noted by its correlated noise increase. Strip widths are varied while the implant width is constant. At right; the explanation for the observed trend. Equipotential lines for the case of congruent edges (a) and with the strip edge stepped back one micron. [21] . . . . . . . . . . . . . . .

3.15 Hysteresis in the SCT. Consecutive IV curves taken during long term bias at 150V. $4 \mathrm{hr}$ intervals in given numerical sequence. [22] . . . . .

3.16 Correlation between the neutron radiation induced deep acceptor states and leakage current increase. The deep acceptor state concentrations are measured directly using DLTS. [23] . . . . . . . . . . . . . .

3.17 Leakage current of recent ATLAS ITk Strip prototype minis (ATLAS12) with and without radiation of different types and fluence. The relative contributions of each type of irradiation to the leakage current increase expected for doses in the HL-LHC. [24] . . . . . . . . . . . . 
3.18 Irradiation effect on charge collection efficiency. Left; collected charge as a function of bias voltage for different neutron fluences. The unit for the numbers next to the curves is $\times 10^{14} n_{\text {eqv }} / \mathrm{cm}^{2}$. Results from two sources (testbeam and beta source) are shown. [25] Right; the evolution of collected charge with fluence for neutron and proton irradiated samples. The NIEL hypothesis is shown. [26] Samples are ITk prototypes (ATLAS07 and ATLAS12) and have been annealed 10min at $80^{\circ}$ C . . . . . . . . . . . . . . . . . .

3.19 The effect of surface damage on interstrip resistance. Results are for recent ATLAS ITk Strip prototypes (ATLAS12). Top, direct measurement of interstrip resistance for different proton fluences. The methodology outlined in Section 5.1.1 is used. Bottom, the interstrip resistance as a function of the total ionizing dose for samples irradiated with protons, neutrons, and gammas. [24] . . . . . . . . . . . .

3.20 The effect or surface damage on interstrip capacitance. Results are from recent ATLAS ITk Strip prototypes (ATLAS12 and ATLAS07). At left, the interstrip capacitance as a function of the bias voltage after various levels of gamma irradiation. Confinement of the induced mirror charge to the thin inversion layer at higher voltages can be seen. At right, interstrip capacitance at very high voltage $(-1 \mathrm{kV})$ as a function of proton fluence on various samples. Saturation to the roughly unirradiated value is evident. [24] . . . . . . . . . . . . .

3.21 Clear advantage of beneficial annealing of $10 \mathrm{~min}$ at $80^{\circ} \mathrm{C}$. Charge collection curves of ITk prototypes (ATLAS07) with and without annealing. Left; reactor neutron irradiation at two fluences. Right; $70 \mathrm{MeV}$ proton irradiation to the fluence of $1.12 \times 10^{15} n_{\text {eqv }} \mathrm{cm}^{-2}$. [26] . . . . .

3.22 Annealing of the irradiation induced space charge concentrations and $\mathrm{CV}$ inferred effective doping concentration. E refers to the $V-P$ defect which removes shallow donor levels. At left, neutron irradiation to a fluence of $5 \times 10^{13} n_{\text {eqv }} / \mathrm{cm}^{-2}$. At right, proton irradiation to $2.3 \times$ $10^{14} n_{\text {eqv }} / \mathrm{cm}^{-2}$. Measured and model predictions are shown, results are for oxygenated float zone substrate. [23] . . . . . . . . . . . . . . . . 101 
3.23 Effect of consecutive annealing periods at $60^{\circ} \mathrm{C}$ of a $23 \mathrm{GeV}$ proton irradiated ATLAS ITk Strip prototype (ATLAS12) to a fluence of $2 \times$ $10^{15} n_{\text {eqv }} / \mathrm{cm}^{-2}$. At left, leakage current. At right, interstrip resistance.

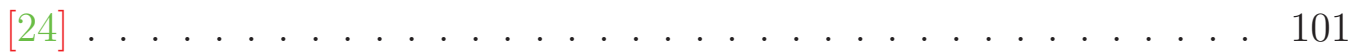

4.1 A photograph of the surface of the ATLAS ITk Strip sensor prototype taken at Carleton University. Most features of the sensor can be seen, refer to the text for the details. . . . . . . . . . . . . . . . . 107

4.2 The ITk Strip endcap's "stereo annulus" geometry. At left; an exaggeration of the geometry depicting the principle. At right; the stereo annulus geometry as applied to the prototype of the innermost sensor, the ATLAS12EC. [1] . . . . . . . . . . . . . . . . . . . . . . . . . 110

4.3 New silicon materials tested for the ITk. Top; the European RD50 Collaboration [27]. Bottom; the Japanese early ATLAS line [4]. . . . 114

4.4 Wafer layouts of the ATLAS ITk Strip prototype sensors. [i] ATLAS05 [28] [ii] ATLAS06 [4], [iii] ATLAS07 [29], [iv] ATLAS12A (axial) [10] [v] ATLAS12M (stereo) [10] [vi] ATLAS12EC [30] [vii] ATLAS17LS [31] 116

4.5 Charge collection of short strip barrel prototypes as a function of irradiation dose and type. Left; nominal operating voltage in the ITk, $500 \mathrm{~V}$ [25]. Right; maximal operating voltage, $700 \mathrm{~V}$ [1]. Beneficial annealing of $10 \mathrm{~min}$ at $80^{\circ} \mathrm{C}$ conducted. Red lines indicate Strip detector's maximum expected fluence. . . . . . . . . . . . . . . . . . . . . 118

4.6 The sensors of the petalet program. Left; schematic showing the petalet structure and sizes. Center; wafer layout for big sensor. Right; wafer layout for top sensors. [32] . . . . . . . . . . . . . . . . .

4.7 Advantage of oxygen enriched silicon in high radiation environments. Full depletion voltage as a function of proton fluence up to $1.1 \times$ $10^{15} \mathrm{pcm}^{-2}$ for oxygen enriched and un-enriched p-type bulk minis. [33]

4.8 The effect of oxygen enrichment on silicon resistivity. Sensor leakage current as a function of fluence for oxygen enriched and un-enriched n-type bulk sensors of size $6.4 \mathrm{~cm}$ square (SCT masks). Samples with different enrichment procedure atmospheres are shown. [34] . . . . . . 
4.9 Radiation hardness superiority of the oxygen enriched $n^{+}-i n-p$ bulk material. Left; at lower proton fluences $\left(3 \times 10^{14} \mathrm{pcm}^{-2}\right)$ the type advantage of p-bulk [35]. Right; at higher proton fluences $\left(1.1 \times 10^{15} \mathrm{pcm}^{-2}\right)$ the advantage of enriched interstitial oxygen content [33]. $\mathrm{y}$-axis units can be considered as arbitrary. . . . . . . . . . . . . . . .

4.10 Charge collection versus bias voltage for p-type mini sensors at three fluences of proton irradiation (as seen). Values are normalized to the pre-irradiation signal size. . . . . . . . . . . . . . . . .

4.11 SCLA effects in early ITk Strip prototype mini sensors. Annealing curves after $23 \mathrm{MeV}$ proton irradiation to thefluences of $1.1 \times 10^{15} \mathrm{pcm}^{-2}$ (left), $3.5 \times 10^{15} \mathrm{pcm}^{-2}$ (left), and $7.5 \times 10^{15} \mathrm{pcm}^{-2}$ (left). The SCLA effect is seen at the higher fluence. [36] . . . . . . . . . . . . . .

4.12 The benefits of space charge limited avalanche. Most probable charge (mean of the charge collection spectrum) as a function of fluence and bias voltage. Left; roughly the maximum fluence expectation for the ITk Strip detector. Right; more typical fluence expectation in the ITk Pixels detector. [37] . . . . . . . . . . . . . . . . . .

4.13 SCLA effects in full size ITk Strip short strip barrel sensor prototypes.Left, the A12 Axial. Right, the sterero strip containing A12 Main. [25] . . . . . . . . . . . . . . . .

4.14 Strip isolation structures of the ATLAS05 (left), ATLAS06 (center), and ATLAS07 (right) prototype sensors. [4] . . . . . . . . . . .

4.15 Electric potential distribution in unirradiated (left) and irradiated (right) TCAD simulated ATLAS07 sensors. Increased field gradient at the surface and reduced depletion depth are observed in the irradi-

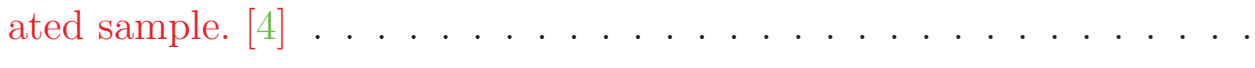

4.16 TCAD simulations for p-stop optimization. Left; electric field strength as a function of distance from the centre of the p-stop. Two interface charge densities are shown with the unit $\mathrm{cm}^{-2}$. Right; electric potential for $6 \mu \mathrm{m}$ p-stop with varying strip pitch in micrometres, interface charge

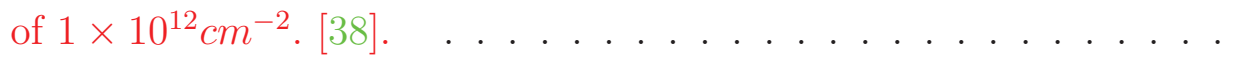


4.17 Development of a radiation hard $n^{+}-i n-p$ strip isolation structure. Left; A05 interstrip currents as a function of bias, structures as given in order of Figure 4.17 top [28]. Centre; interstrip resistance vs. capacitance for the A07 structures (Figure 4.17 bottom) irradiated to $1 \times 10^{13} n_{\text {eqv }} \mathrm{cm}^{-2}$. Right; for the same irradiation interstrip resistance as a function of the isolation doping (sum of spray and stop). [39] . .

4.18 Punch through protection development for the HL-LHC. Top left; the ATLAS07 variants [29]. Bottom left; the small size test sensors PTP variants [40]. Right; the ATLAS12 variants [24]. . . . . . . . . . . .

4.19 TCAD simulated comparison of advancing radiation hardness in PTP design. Left; extended electrode implemented in the ATLAS07 Z4D, unirradiated [29]. Right; the full gate structure implemented in ATLAS12 full size, irradiated to $1 \times 10^{15} n_{\text {eqv }} \mathrm{cm}^{-2}$. Strip implant at $-50 \mathrm{~V}$ for both, refer to Figure 4.18 for structures. [38] . . . . . . . . . . . .

4.20 Radiation hard PTP structures of the ATLAS12M sensor submission. Current between rail and implant as a function of voltage for the six designs seen in Figure 4.18. A proton irradiated sample with a fluence of $1 \times 10^{15} n_{e q v} \mathrm{~cm}^{-2}$ is tested at $-20^{\circ} \mathrm{C}$ and $1000 \mathrm{~V}$. [24] . . . . . .

4.21 An example layout of the edge structure test diodes. The circle corresponds to the focused view offered by Figure 4.22 left. Outer dimension of the diode is $4 m m . \ldots . \ldots . \ldots$

4.22 Guard ring structures tested in the diode test sensors. Left; structure designs denoted by ring multiplicity and feature width [41]. Right; microdischarge onset voltage for the different structures as a function

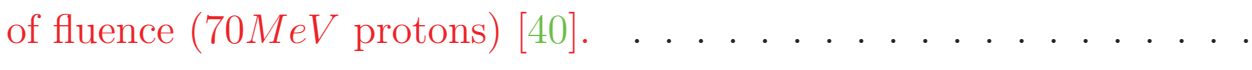

4.23 Results of edge width tests conducted on the diode test sensor batch. Left; microdischarge onset voltage as a function of edge width for $\mathrm{p}$ and $n$ type sensors, the saturation point is $1000 \mathrm{~V}$. Centre; IV curves for variant edge width, from $233 \mu \mathrm{m}$ to $1211 \mu \mathrm{m}$ (legend: wafer- total edge width in micron). One guard ring is preferred from the W3-403 and W3-403-2GR data [41]. Right; onset voltages for microdischarge (MD) as a function of edge width and guard structure for irradiated

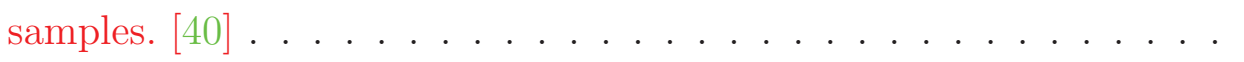


4.24 Slim edge implementation. Photograph of the corner of an ATLAS12 sensor surface with conventional cut. The slim edge dicing street is shown in yellow. [42] . . . . . . . . . . . . . . . . . 136

4.25 Slim edge dicing effect on the IV curve of the ATLAS12M and ATLAS12A sensors. Left; sensors before slim dicing (sensors with conventional edge of $\simeq 910 \mu \mathrm{m})$. Right; sensors after slim edge cut $(\simeq 450 \mu m)$. [42] . . . . . . . . . . . . . . . . . . .

4.26 Examples of ganging schemes implemented in the ATLAS12 $1 \mathrm{~cm} \times$ $1 \mathrm{~cm}$ sensors. Light blue denotes the interstrip connections. Left; DC ganging. Right; AC ganging. [41] . . . . . . . . . . . . . . . 138

4.27 Embedded pitch adapter variants studied in the 2016 testbeam data. [43] 139

4.28 Microdischarge and TCAD assisted large area, stereo strip, sensor mask refinement. Left; hot-electron image of the systematic microdischarge. Centre; the mask feature causing the problem. Right; the mask fixes successfully implemented. [29] . . . . . . . . . . . . . . . .

5.1 Equivalent circuitry for the "full striptest" conducted on a subset of the ITk sensors. . . . . . . . . . . . . . . . . . . . . . . . . . . . 147

5.2 Equivalent circuitry for the interstrip tests conducted on a subset of the ITk sensors. Left; the AC interstrip capacitance measurement. Right; the DC interstrip resistance measurement. . . . . . . . . . . . 148

5.3 Photograph of one of the first ATLAS12EC prototypes ever produced. Taken at Carleton University. . . . . . . . . . . . . . . . . . 153

5.4 Photograph of the ATLAS07 at Carleton University. It is seen wirebonded into the custom jig described in Section 5.4.2 which is vacuum sealed to the chuck of the Karl Suss probestation (Section 5.4.1). . . . 154

5.5 Examples of visual inspection results obtained at Carleton. . . . . . . 157

5.6 The Carleton custom metrology jig featuring an adjustable-tilt two stage design and geometrical accommodation of all sensor geometries. At left; a photo of the jig with an ATLAS12EC in the MuAnalysis LSM. At right; schematic of the geometry accommodation feature. . .

5.7 Metrology results for sixteen ATLAS12EC prototypes. Data collected at MuAnalysis by Carleton practitioners. . . . . . . . . . . . . . . . 160 
5.8 Histogram of the ATLAS12EC flatness results shown in Figure 5.7. With a mean of $54.4 \mu \mathrm{m}$ and standard deviation of $6.5 \mu \mathrm{m}$ very good consistency that is well within the $200 \mu \mathrm{m}$ specification was observed. Data collected at MuAnalysis by Carleton practitioners. . . . . . . . 160

5.9 The Carleton University electrical test setup at an earlier testing stage in the development. A number of components can be seen; the Karl Suss probestation with the Keithley instruments atop the black enclosure. The LCR meter, largest patch panel, and data acquisition laptop are seen at the right. These components are discussed throughout Section 5.4. . . . . . . . . . . . . . . . . . 162

5.10 The Carleton University custom test jig. Designs modified and jig fabricated on-campus. Left; photo of the jig with the ATLAS07 short strip barrel prototype wire-bonded in. Right; schematics of the design developed with the Physics Department design staff. . . . . . . . . . . 166

5.11 The custom designed and fabricated patch panels in use at the Carleton University sensor test facility. . . . . . . . . . . . . . . . . .

5.12 Examples of the patch panel connection schematics produced for the QC tests given in Section 5.1.1. Top; full striptest (corollary to equivalent circuit of Figure 5.1). Centre; interstrip capacitance connections (Figure 5.2 left). Bottom; interstrip resistance (Figure 5.2 right). . . . 168

5.13 Examples of the Arduino based environmental monitoring system. Left; photograph of the mostly breadboard based circuitry used in the six SHT setup. Right; example results of humidity over time in the newly developed LabVIEW code. The green line represents an SHT outside of the enclosure. . . . . . . . . . . . . . . . . .

5.14 Visualization of the effect of $I_{\text {leak }}$ temperature correction using ATLAS12EC data obtained at Carleton University. Top; IV curves obtained at $21-28.5^{\circ} \mathrm{C}$. Bottom; the same data normalized to $20^{\circ} \mathrm{C}$. An attempt to use equivalent linear y-scales has been made. . . . . . . .

5.15 A comparison of temperature normalized IV curve results for the three most recent sensor submissions. Top; ATLAS07 [44]. Centre; ATLAS12 [1,42]. Bottom; ATLAS12EC (data obtained at Carleton). . . 176 
5.16 Histogrammed leakage currents results of the Carleton ATLA12EC data at various voltages during IV curve. Top; 600V ITk operation proxy. Bottom; QA interesting voltages of $200 \mathrm{~V}$ (left) and $1000 \mathrm{~V}$ (right). . . . . . . . . . . . . . . . 177

5.17 The inverse relation observed between humidity and microdischarge onset voltage is seen in this plot of sequential IV curve measurements at decreasing humidities. Carleton data for the ATLAS12EC sensor W048 is shown. . . . . . . . . . . . . . . . . . .

5.18 A comparison of CV curve results for the three most recent sensor submissions. Top plot; ATLAS07 with $V_{f d} \simeq 200-250 \mathrm{~V}$ [45]. Center plot; ATLAS12 with $V_{f d} \simeq 320 V[1,42]$. Bottom plot; ATLAS12EC with $V_{f d} \simeq 300 V$ (data obtained at Carleton). . . . . . . . . . . . 180

5.19 Histogrammed full depletion voltage results of the Carleton ATLAS12EC data. CV curve inference is used to obtain $V_{f d}$. An average full depletion voltage of $302.7 \mathrm{~V}$ and an RMS of $1.8 \mathrm{~V}$ is obtained for the 15 sensor sample. . . . . . . . . . . . . . . . . .

5.20 The effective doping concentration profile as a function of bias voltage for 15 ATLAS12EC sensors. A small batch-to-batch variation is seen in the step-like feature located in the range $60-80 \mathrm{~V}$. Data collected at Carleton University. . . . . . . . . . . . . . . . . . . .

5.21 Combined data results for leakage current long-term stability tests of two ATLAS12EC sensors. Red is leakage current in $\mu A$, green is temperature in ${ }^{\circ} \mathrm{C}$, and blue is humidity in $\% R H$. Top; the humidity sensitive W048 at $25 \pm 5 \% R H$ for $18 \mathrm{hr}$. Centre; the humidity sensitive W048 at $36 \pm 5 \% R H$ for $24 h r$. Bottom; the average (insensitive) W042 sensor at $36 \pm 2 \% R H$ for $12 h r$. Temperature corrections are not applied and a temporal offset exists in the cyclic environmental monitoring of the first plot (trends in reality line up). Data collected at Carleton University. . . . . . . . . . . . . . . . . .

5.22 Hysteresis in an ATLAS12EC sensor (the humidity sensitive W048). OLD refers to April of the same year. Dry storage conditions between tests were variable but recorded. Data collected at Carleton University. 185 


\section{Chapter 1}

\section{The Inner Tracker Upgrade}

What are the most fundamental constituents of matter? How do they acquire mass? Form galaxies, stars, and planets? What are the forces that allow these stars to ignite and lead to the formation of life? The forces that control the exchanges of energy and information that dictate the evolution of the universe (and our email folders)? What is the 'dark' sector that makes up over $90 \%$ of the energy-mass composition in the universe? Is there anything else?

These are all questions that the ATLAS Inner Tracker (ITk) is being built to answer. It will be the new central tracking system installed into the ATLAS detector at CERN's Large Hadron Collider (LHC) as it enters into its next generational phase. This upgrade to the LHC machine, already able to produce the highest energies attainable by humankind, will allow a significant increase in the rate of the collisions as well as an extension of the project lifetime. Resulting in the collection of ten times more premier physics data than was planned for the LHC at its conception. This translates to increased sensitivity to all interactions within the LHC's energy capability; both discovered and, potentially, undiscovered. Beginning in 2026 and with a planned lifetime of at least ten years, the "high luminosity"-LHC (HL-LHC) upgrade (and ITk with it) will be at the forefront of high energy physics understanding for the coming decades.

The topic of this Master's thesis is the silicon microstrip detectors located in the outer portion of the ITk. In this chapter the motivation and basic background is presented. The goals of the ITk are intimately entwined with those of ATLAS and the HL-LHC. Questions such as those posed above are the basis for particle accelerators whose heavy handed nature has been successful in development and evaluation of the particle physics benchmark theory, the Standard Model. The importance of the 
LHC and HL-LHC are put in this context in Section 1.1. A quick look at the ATLAS detector follows in Section 1.2. This is preparation for the ITk specific motivation in Section 1.3 where a brief discussion of the major issues endemic in current detector is given. The chapter comes to a close in Sections 1.4 and 1.5 with an overviewing scrutiny of the Inner Tracker (ITk) and its expected performance (respectively).

With this elementary material covered the following discourse finds good footing as it tracks through the specifics of the Strip portion of the ITk detector (Chapter 2 ), the theory of the silicon microstrip sensors (Chapter 3), their past development (Chapter 4), and their current development (Chapter 5).

\section{$1.1 \quad$ HL-LHC}

The ITk Strip sensors will operate in the astronomically harsh conditions of the HLLHC. The High Luminosity Large Hadron Collider (HL-LHC) is the end product of a number of upgrades on the LHC accelerator complex (Figure 1.1) that will allow an increase in the collision rate by a factor of seven and a half from its original design value. A nearly threefold increase in the lifetime of the project results from the upgrade scheme, the last (projected) decade of which will be entirely full energy, high luminosity (high collision rate) operation. The end goal is the collection of ten times more $O(10 \mathrm{TeV})$ data than originally intended for the LHC. This order of magnitude increase may illuminate some phenomena just beyond our current sensitivity. Undoubtedly, it will allow a finer investigation of known phenomena, a natural example being the newly discovered Higgs boson. Considering the Higgs' singular importance in the question of mass (and correspondingly in the Standard Model as a whole) a more precise knowledge of the Higgs couplings to the other fundamental particles (via reduction of statistical uncertainties) is one of the most promising, known leads to new physics in the high energy field.

In this section the general inward trend of the thesis is begun at the level of the LHC machine. Necessary Standard Model basics are given (Section 1.1.1) followed by discussions on the motivations for the current accelerator (the LHC, Section 1.1.2) and its future high luminosity upgrade (the HL-LHC, Section 1.1.3). The upgrade schedule (Section 1.1.4) wraps up the section and LHC machine focused examination. 


\subsubsection{The Standard Model and Its Experimental Handles}

In particle physics the goal is to determine the most fundamental constituents of matter and the forces that dictate their interaction. The current understanding is formulated in "the Standard Model". Developed in the 1960s and 70s this theoretical model now stands as one of the most stringently tested in existence. It is a perfect example of the necessary interplay that experiment and theory assume. The first half of the 20th century saw the atom replaced by electrons, protons, and neutrons as the most fundamental bits of matter. This modern elementary school understanding was a huge advance especially when it incorporated the quantum mechanical description of the micro-scale that was developing along side it. However, in the 1930s to 1950s a large number of new particles were discovered leading to the development of the concept of a "particle zoo" (a space filled with wild, exotic, poorly understood creatures). A new theoretical understanding was required.

The Standard Model incorporated the existence of quarks to breakdown the wild variety of the zoo into a simple, natural hierarchy. It is built in the mathematical framework of relativistic quantum field theory. The particles are considered as quantized excitations in dynamical fields that pervade all of space-time. The universally invariant laws dictating their evolution are realized naturally via Noether's theorem leading to the gauge character of the Standard Model formulation (ie. the fundamental forces accommodated therein are described by their own gauge field) [11]. The Standard Model utilizes SU(3)xSU(2)xU(1) gauge symmetries which correspond approximately to the three fundamental forces of strong, weak, and electromagnetism. The Standard Model is discussed more in context to the mass-imbuing Higgs mechanism in Section 1.5.3.

The underlying symmetries that defined the new theory and that allowed the logical understanding of the particle zoo provided predictions for new particles. Experimental evidence for these undiscovered particles was found in following experiments or even in existing data. The Higgs boson is one example of the former. Over the decades since its inception 1000s of high precision experimental measurements have corroborated the Standard Model understanding. However, the model is not complete. It does not explain dark matter, why the particles have the largely variant masses they do (the hierarchy problem) or why there is more matter than antimatter. Furthermore, it does not describe the fourth fundamental force, gravity, and is in-

compatible with its tested theoretical understanding, general relativity. New physics 
beyond the standard model exists and its discovery is the main goal for modern experiments such as the LHC and HL-LHC.

To advance understanding requires experimental verification which requires a knowledge of the available handles on the phenomena of interest. Here, the phenomena is that of quantum mechanical scattering interactions. In modern stimulated particle physics two aspects define an experiment's potential for new discovery; the energy at which they can induce an interaction and the amount of interactions at that energy they can attain.

Through the energy-mass equivalence relation $\left(E=m c^{2}\right)$ the first of these defines the particles which can be produced in the interaction. Due to the second law of thermodynamics and the existence of decay mechanisms, the heavier particles are rarer and shorter lived, quickly decaying to the more stable and thus plentiful and well understood relatives (protons, neutrons, electrons, muons...).

The nature of the quantum mechanical interaction is statistical which defines the usefulness of the second parameter, that of the number of interactions. A vast number of interactions must be stimulated to observe a statistically significant number of rare events that may lead to new physics.

The two aspects of energy and statistics are wedded in collider physics where intense beams made of large numbers of massive particles are accelerated to nearly light speed and made to collide with each other or a target. The story of the LHC and HL-LHC can be understood thoroughly in consideration of these two simple factors.

\subsubsection{The LHC and Its Importance}

The LHC's main motivation was interaction energy. The significance of being able to produce the highest energies is easily understood from the above discussion. This limit of human ability is called the "energy frontier", it currently sits at the $13 \mathrm{TeV}$ centre of mass collisional energy that the LHC is currently operating at. Particles and states of matter that have not existed since a fraction of a billionth of a second after the big bang are re-created in the hearts of the four detectors that sit on the $27 \mathrm{~km}$ ring. ${ }^{1}$ This explosive atmosphere is situated serenely under the vineyards and sparse population of the French-Swiss border near Geneva.

\footnotetext{
${ }^{1}$ Energies and particles that have not existed outside the quantum time-scales defined by the uncertainty principle.
} 


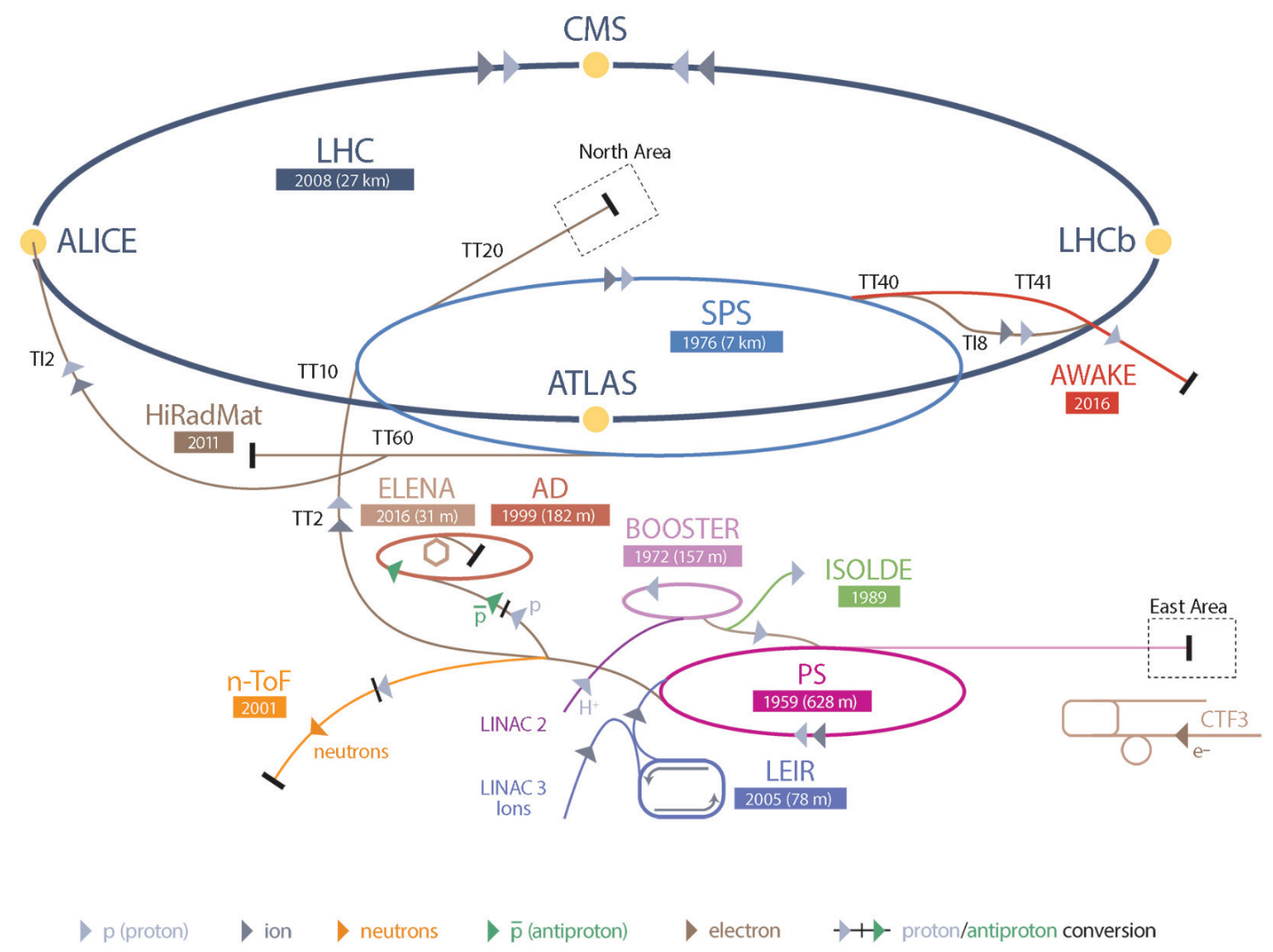

Figure 1.1: Schematic of CERN's LHC starring accelerator complex. Accelerators, beam lines, and (some) detector experiments are shown. The LHC protons are generated from hydrogen in Linac 2. They then pass through the Proton Synchrotron (PS), the booster, and the Super Proton Synchrotron (SPS) before reaching the LHC main ring. Colors denote accelerated particle type. [5]

An astonishing collision rate is coupled to the $14 \mathrm{TeV}$ energy capability. Thousands of bunches of billions of protons are counter-rotated and made to intersect forty million times per second at the four detector points. This produces tens of interactions per 25ns spaced "bunch crossing" and has allowed the LHC to facilitate strides in humankind's fundamental understanding. One linear and three circular accelerators are required to bring the particles up to the speed required by the LHC's thousands of $O(10 T)$ superconducting bending magnets. The CERN accelerator complex is shown in Figure 1.1 [5].

The most notable of the LHC discoveries is that of the $125 \mathrm{GeV}$ Higgs boson in 2012. A discovery whose significance is heralded by the 2013 Nobel Prize being 
awarded to its theoretical proponents. First defined in the 1960s it is one of the keystone pieces of the Standard Model; a predicted consequence of the conventionally accepted method by which particles gain mass (electroweak symmetry breaking, discussed further in Section 1.5.3). [11] It remained undiscovered for half a century mostly (but, as will be seen, not entirely) because the experiments over that period did not have the energy to produce it. Experimental verification of the Higgs boson was one of the more cogent motivations for the LHC since it directly helps answer some of the fundamental questions posed at the start of this discussion.

\subsubsection{The HL-LHC and Its Importance}

The HL-LHC's motivation is number of high energy interactions (statistics). In the quick and dense environment of an LHC bunch crossing the decay products from the short lived, rare particles must be picked out of the background of more common events. Careful design in the detector composition, structure, and the driving algorithms can only mitigate this challenge. Ultimately, collider physics is a field highly driven by statistics. Experiments such as the TeVatron and Large Electron-Positron Collider (with nearly $2 \mathrm{TeV}$ and $209 \mathrm{GeV}$ maximum centre-of-mass collisional energies respectively) preceded the LHC and had the energy capability to produce the Higgs. However, in the end they lacked the statistics necessary to announce a discovery. The TeVatron even detected a signal coincident with the $125 \mathrm{GeV}$ Higgs boson found at the LHC. However, after analysis of the full dataset statistical significance on the signal was placed a good deal shy of the five sigma mark conventional in the field for a discovery.

The collision rate and period over which the experiment can be maintained dictate the total number of interactions produced. In collider physics these are expressed in terms of luminosity; instantaneous luminosity quantifies the collision rate while the (time) integrated luminosity gives the total number of collisions. The dimensions of inverse area (and its accumulation rate) are easily understood when considering that the quantum mechanical production interactions at play in the stimulated environment of a collider fall under the classification of scattering phenomena. In scattering theory (the study of interacting PDE solutions) the probability of an interaction naturally finds units of area in the interaction 'cross section'. At the quantum scale of particle physics phenomena these area are correspondingly minute; a new con-

venience, the barn (b) is used and is equivalent to $10^{-28} \mathrm{~m}^{2}\left(1 \mathrm{~b}=10^{-28} \mathrm{~m}^{2}\right)$. The 

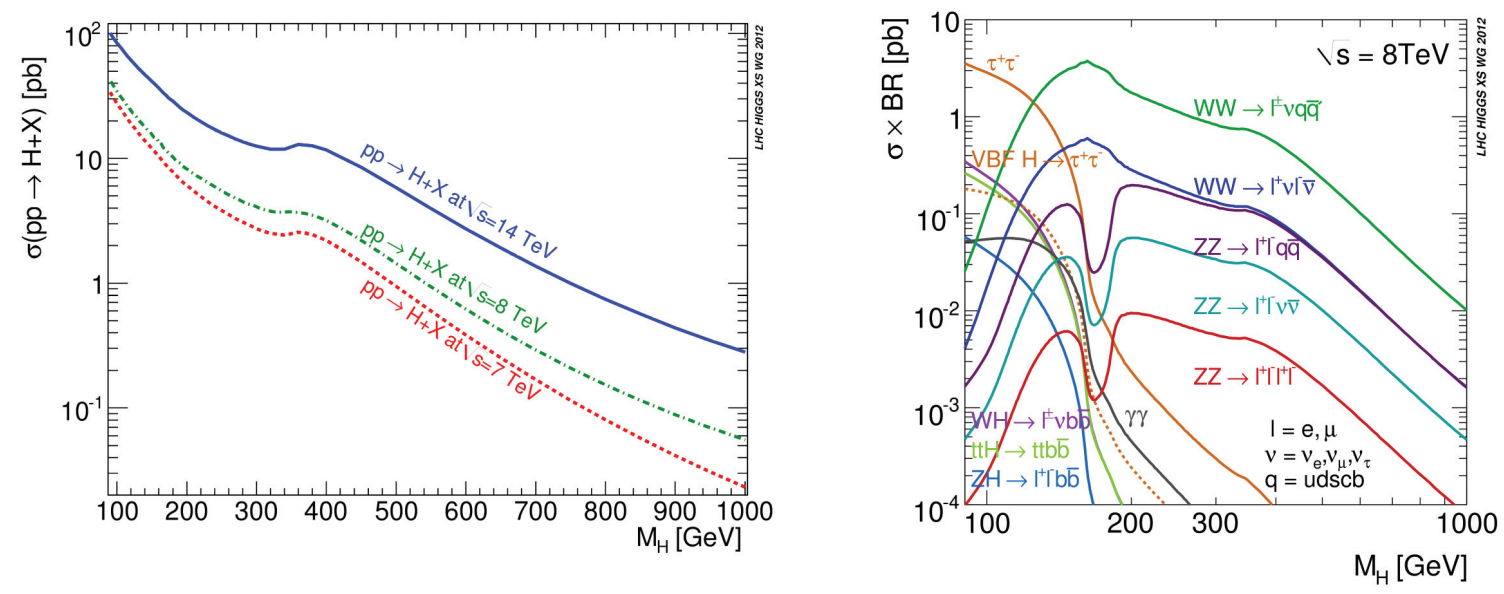

Figure 1.2: Importance of statistics (integrated luminosity). Left; Standard Model predictions for the Higgs boson total production cross-section as a function of Higgs mass and interaction energy. Right; Standard Model precictions for the Higgs boson decay channels as a function of Higgs mass; the 'detectable' Higgs boson cross-section (production c.s. times branching ratio). [6]

colliding beams' cross section provides the corollary area over which the interaction could occur and thus obtains units of inverse barn. This notation allows the number of interactions at a collider to be easily predicted from theory as the product of the detection cross section and the experimentally obtained integrated luminosity.

As Figure 1.2 shows the more interesting interactions are small even by the minute barn measure. For example, the Standard Model predictions for the Higgs boson production are on the scale of picobarns (at LHC energies, see Figure 1.2 left). Subattosecond lifetimes predicted for particles like the Higgs mean that the only practical signal is detectable from the decay products only. For the heavier particles many decay channels are allowed in the Standard Model, this is shown in the right side of Figure 1.2 for the $125 \mathrm{GeV}$ Higgs at $8 \mathrm{TeV}$ centre-of mass interaction energy. As can be seen by comparing the left and right side of Figure 1.2 this consideration causes significant reductions in the detectable cross section (right) from that of the pure production mechanism (left) [6].

So far observations at the LHC have closely matched the theoretical predictions of the Standard Model. This includes the properties of the $125 \mathrm{GeV}$ Higgs boson discovered in 2012. The lesson in history given by the TeVatron and Large Electron Positron Collider (LEP) succinctly summarize the motivation for the HL-LHC upgrade. The $14 \mathrm{TeV}$ energy capability of the LHC is a significant increase from the 


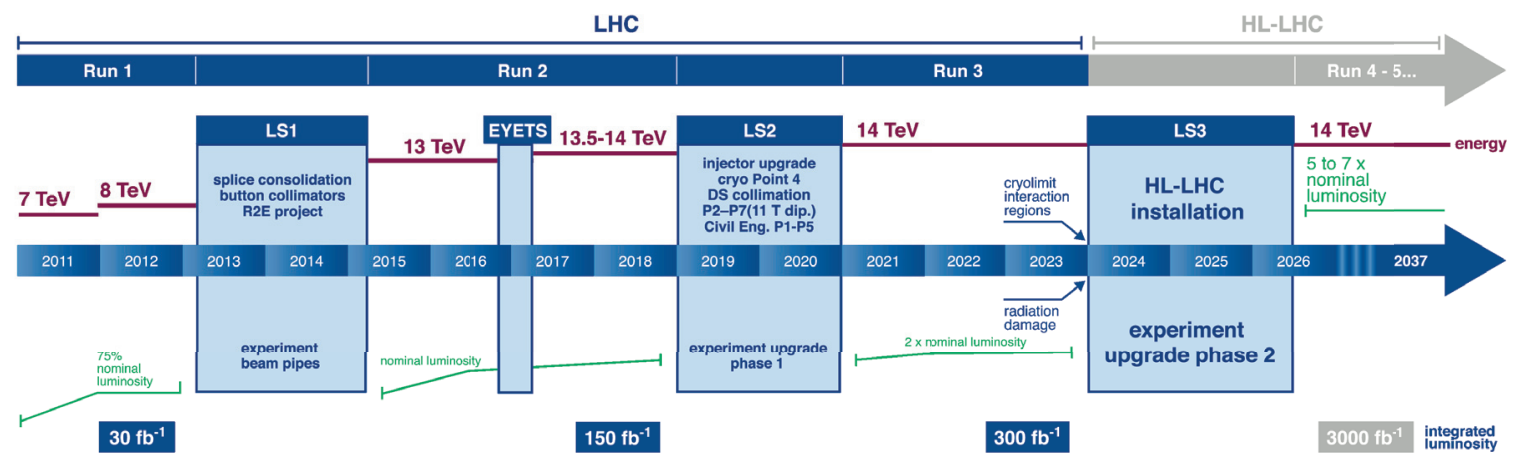

Figure 1.3: Baseline program for the LHC including the HL-LHC phase. The green line signifies instantaneous luminosity, while the red signifies the energy. Nominal luminosity is the LHC nominal; $1 \times 10^{34} \mathrm{~cm}^{-2} \mathrm{~s}^{-1}$. LS stands for long shutdown. [1]

preceding accelerators; the first significant step into the tera-electronvolt scale. With the high luminosity upgrade's promise of an order of magnitude more premier physics data the LHC facility's enormous potential will not be squandered $[1,3,5,7,46]$.

\subsubsection{HL-LHC Upgrade Schedules}

The LHC upgrade scheme is shown in Figure 1.3 [3]. Consisting of three "long shutdown (LS)" periods (each of approximately two years in duration) it will allow the LHC's original planned lifetime of ten years to be extended until at least 2037, an extra 17 annum. The upgrade sees a ramp up in both energy and instantaneous luminosity over this period. The first long shutdown occurred from 2013 to 2015 and saw extensive maintenance of the entire LHC accelerator complex (Figure 1.1) with a focus on the magnet issues that had already cost nearly two years of operational time (2008-2010). Only recently has the LHC passed the point when its energy and luminosity were prescribed to meet their design values of $14 T e V$ and $1 \times 10^{34} \mathrm{~cm}^{-2} \mathrm{~s}^{-1}$ (respectively). Current operational records sit at $13 \mathrm{TeV}$ and $1.58 \times 10^{34} \mathrm{~cm}^{-2} \mathrm{~s}^{-1}$ (with the lower energy chosen for the start of 2017 operation in lieu of the training period required for the energy increase) [5].

The second long shutdown beginning in early 2019 will see an increase in beam collimation ability as well as fundamental upgrades to the LHC injector facilities as seen in Figure 1.1 [7]. A target $2 \times 10^{34} \mathrm{~cm}^{-2} \mathrm{~s}^{-1}$ will result for "Run 3" of data collection (see Figure 1.3) by the end of which $300 \mathrm{fb}^{-1}$ of premier proton-proton 
collisions will be collected.

The final preparations for the HL-LHC operation will commence in 2024. The modifications to the LHC, extensive enough to warrant the HL- prefix, will allow the sizable increase to a nominal target instantaneous luminosity of $5 \times 10^{34} \mathrm{~cm}^{-2} \mathrm{~s}^{-1}$. A ten year operation at this luminosity will produce the targeted increase to $3000 \mathrm{fb}^{-1}$. Since all components associated with the HL-LHC machine will have a minimum design margin of $50 \%$ another performance scheme has also been considered (and is becoming the predominant choice since equipment is often tested beyond this value). The "ultimate" HL-LHC operational parameters feature a $7-7.5 \times 10^{34} \mathrm{~cm}^{-2} \mathrm{~s}^{-1}$ instantaneous luminosity which would allow $4000 \mathrm{fb}^{-1}$ of data to be collected in the ten year HL-LHC target lifetime.

The method by which the high luminosity upgrade is achieved is dominated by three factors; an increase in the number of protons in each of the bunches that circulates the LHC ring, a decrease in the size of the interaction region, and the use of a technique to maintain more constant collision rates ("luminosity leveling"). These advantages are gained from a number of major upgrades to the LHC machine. Some major examples include completing the renovations of the (pre-)accelerator complex that were begun in the previous long shutdown, and on the large ring, a significant upgrade to both the bending and collimating magnet systems as well as their supporting services [5].

These machine upgrades will result in smaller, more populated bunch crossings in the hearts of the four detectors on its ring. A significant result of this is the increase in the number of collisions per bunch-crossing. In LHC terminology this is called "pileup" and is given the character $\mu$. The five to seven and a half increase in instantaneous luminosity will cause the number of proton-proton collisions per $25 \mathrm{~ns}$ bunch crossing to rise from a transitory 40 (average 24) to a sustained $140-200$. A simulation of a single bunch crossing in the ATLAS detector is seen in Figure 1.4. It allows this significant effect to be visualized [7]. It should be noted that pileup refers only to the possibly interesting, hard-scatter collisions, a vast number of soft interactions also accompanies this and is similarly increased in the HL-LHC.

The boost to a more sustained nature in the collision rate at the HL-LHC is due to a technique called "luminosity leveling" [1,47]. The technique involves co-opting the normal exponential-like decaying manner of a proton injection at the LHC by applying a compensatory increase in the collision intensity [47]. This method which targets 


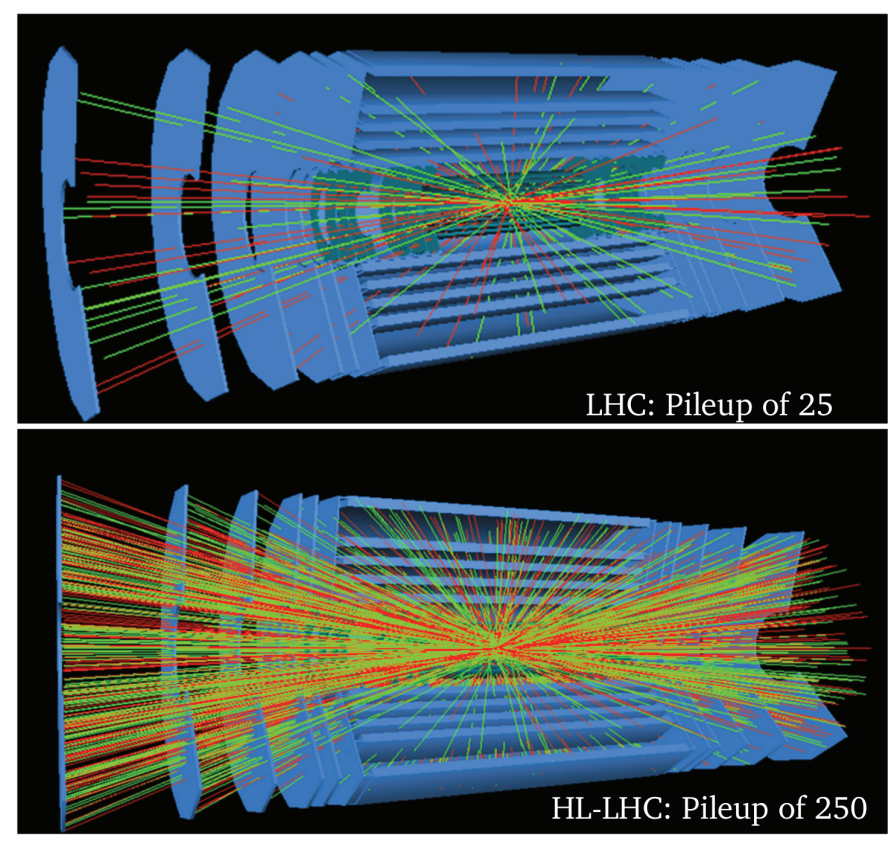

Figure 1.4: Pileup simulations in conditions akin to the LHC and current ID (top) as well as HL-LHC and ITk (bottom). The detector shown in blue is an old layout of the ITk. [7]

integrated luminosity rather than its peak instantaneous counterpart is an integral consideration for the HL-LHC's operational targets. Since the peak luminosity is co-opted this mode also augments the magnitude of the high luminosity upgrade in the context in which it usually discussed.

The HL-LHC will use state-of-the-art technology developed specifically for the fundamentally unique challenges that it entails. A compensatory response will be required from the detectors that sit poised to capture the resultant interactions. The foremost of which (in interest of this thesis) is ATLAS, one of the two general purpose detectors on the LHC's main ring. It is seen the grander scheme of the LHC accelerator machine in Figure 1.1. ATLAS plays as a minor stop on the inward journey towards the ITk Strip sensors, necessary since it is the larger detector system into which the ITk will be installed during HL-LHC operation.

\subsection{The ATLAS Detector}

A Toroidal LHC ApparatuS (ATLAS) is the largest detector in particle physics. At 7000 tonnes and roughly cylindrical with approximate dimensions of $46 \mathrm{~m}$ by $25 \mathrm{~m}$ by 


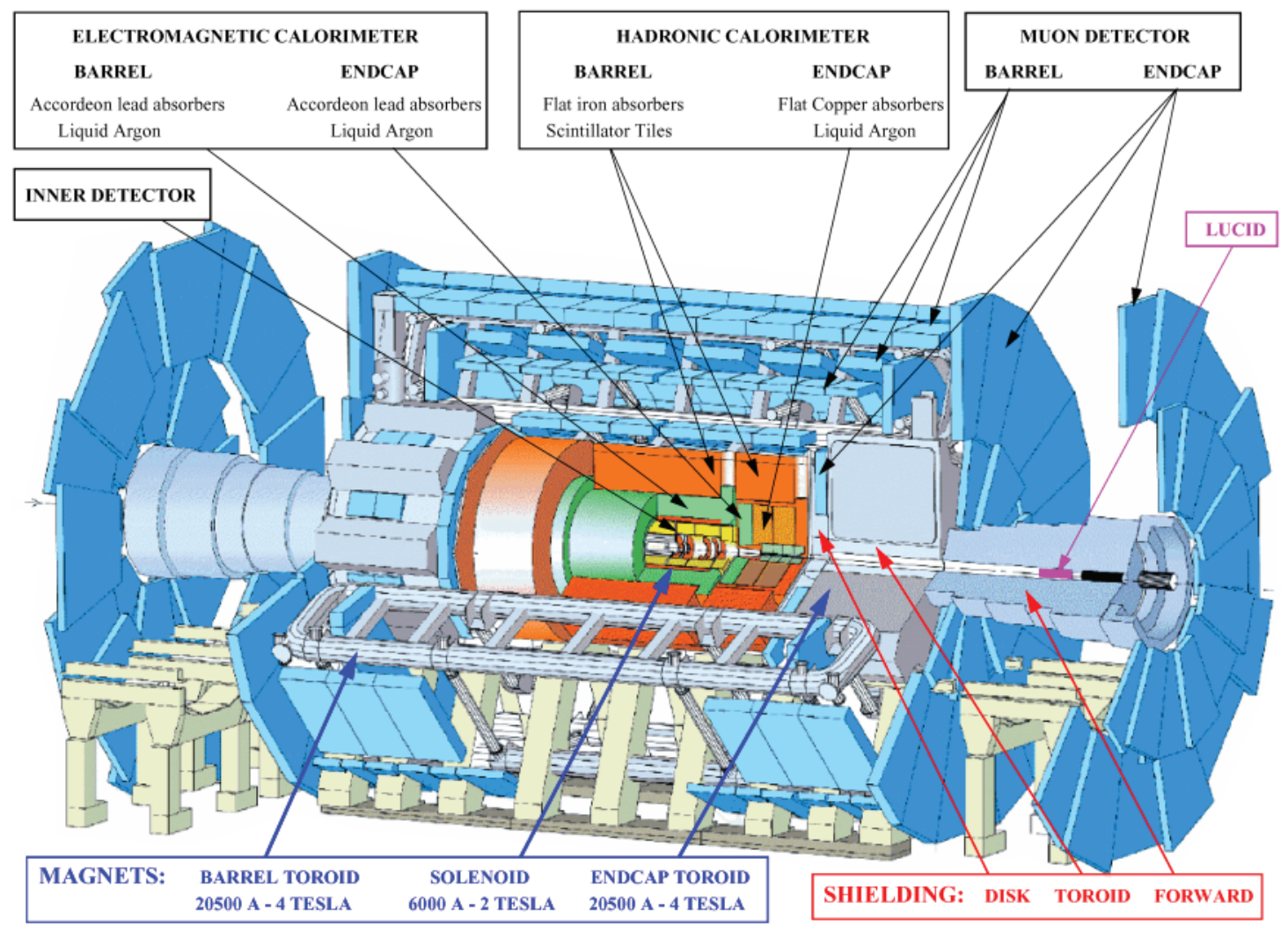

Figure 1.5: Simplified schematic of the LHC's ATLAS detector (its current iteration). The main sub-detector systems are shown. [8]

$25 m$ it is a mammoth achievement in humanity's scientific and engineering ability. It was instrumental in the uncovering of the Higgs boson an honor it shared with the one other general purpose detector on the LHC ring (the Compact Muon Solenoid, CMS).

\subsubsection{Overview}

A simplified17 schematic of the ATLAS detector is shown in Figure 1.5 [8]. It is designed to detect as many different decay products as possible. This conventionally leads to an onion-like geometry that is extended into a cylinder by the collisional nature of the beams and construction feasibility considerations.

Different detector technologies are placed at increasing radii from the interaction point each with complementary functionalities for particle detection. The heart of the ATLAS detector is an ionizing particle tracker, called the Inner Detector (ID), it 
is shown in yellow in Figure 1.5. Ringed by a $2 T$ solenoid magnet (seen in orange) it provides key particle momentum and identification information. ATLAS' innermost detector upgrade is the focus of this Master's work and will be discussed more thoroughly throughout the remainder of this document.

The tracker is shelled by two calorimeters which allow high level energy and particle identification by exploiting the characteristic stopping power coefficients of the collision products. An electromagnetic calorimeter (shown in green) halts the lighter (leptonic and photonic) matter. It is enveloped in the denser hadronic calorimeter (shown in orange) which catches the heavier quark composite particles (hadrons) that are produced via decays, the energy-mass equivalence, and the strong force. ${ }^{2}$

The inner ATLAS sub-detectors (approximately $9 m$ in diameter and $12 m$ in length) are engulfed by the muon tracking system, seen in blue in Figure 1.5. The heavy-electron-like muons offer a singular advantage in collider physics detectors like ATLAS. They are produced almost exclusively in interesting events and they are the only detectable particles to make it past the calorimeters (they act as minimum ionizing particles or "mips"). A very pure signal can be achieved in muon channels due to this feature. The enormous $4 T$ toroidal magnets (shown in grey) that give ATLAS its name provide the bending mechanism for momentum and charge inference in this case.

\subsubsection{ATLAS Upgrade Schedule}

The ATLAS detector will follow an ambitious upgrade plan. For logistical purposes, the upgrade scheme progresses in step with that of the LHC accelerator complex. This tripartite structure was laid out in Figure 1.3. The intense detector development is required to keep ATLAS fully functional throughout the full 27 years and 3000 inverse femto-barn of data. The main concern for the detectors in the next generation LHC is the "end-of-lifetime" performance occurring during the last few years of operation. Combining the ability to handle the increase in collision intensity with the effects accrued from years of operation in a highly radioactive environment is not an easy task. As will be seen this task falls predominantly on the ITk and its novel, large area, radiation hard sensors.

During the first long shutdown, completed in 2015, the main ATLAS focus was

\footnotetext{
${ }^{2}$ Leptons and quarks are the two main groups of matter particle in the Standard Model. This is joined by the force transmitting bosons.
} 
the insertion of a new layer immediately adjacent to the beam line. ${ }^{3}$ With high granularity this well placed, minimally disruptive upgrade, given the title Phase-0 in the ATLAS canon, prepared the detector for its current operation.

The Phase-I upgrade planned for the 2019-2020 break (see Figure 1.3) will see further modifications for higher luminosity environments. Fundamental upgrades to the trigger system (allowing hardware level track finding) and a tailored upgrade to the muon system (offering more significant tracking advantages) are the main operations during this long shutdown.

For the ATLAS detector the most critical part of the high luminosity upgrade is the Inner Tracker which is a full replacement for the current innermost tracking system, the Inner Detector (ID). It is the main operation planned for Phase-II of the ATLAS upgrade scheme (accompanying upgrades to the calorimeter and trigger systems). This final phase of modifications will occur in conjunction with, and in preparation for, the advancement of the LHC into its high luminosity phase. As seen in Figure 1.3 this transformation is planned for the long shutdown period of 20242026. The next section will show that the introduction of the ITk is a necessary exchange, required for the ATLAS to perform under the HL-LHC's next generation targets in instantaneous and integrated luminosity.

\subsection{The Inner Detector and Its Replacement}

The construction of ATLAS and the LHC, roughly a decade and half ago, pushed the boundaries of experimental particle physics. The high luminosity modifications will push the LHC operation into a new realm. At the radii closest to the interaction point the inner tracking system is most sensitive to both the accumulation of radiation damage (integrated luminosity) and the increase in collision intensity (instantaneous luminosity). The ID which was developed in line with the forefront of detector knowledge in the early 2000s will not only have exceeded its design limits at HL-LHC install, it cannot be expected to cope with a fifteen plus year advancement in accelerator technology. As a result its complete replacement is an absolute necessity for the operation of ATLAS during the HL-LHC. The Inner Tracker (ITk) is the answer.

The considerations in design of the ID as well as its shortcomings, and the design of

\footnotetext{
${ }^{3}$ Within the pixel layers of the ID, see Section 1.3.1.
} 
its replacement revolve around the coupled luminosity aspects. Detector technology must be carefully cultivated for radiation hardness with a layout, granularity, and readout that can cope with the intensity and speed of the collision environment for the experiment duration. These four important factors drive this and the following sections' discussion on the ID's replacement (and much of the other detector related analysis).

The main arguments for the Inner Detector replacement are given in Table 1.1. The layout of the existing detector is summarized in Section1.3.1. This is followed by the turning point arguments which contrast the current system's design limits and the HL-LHC projections, Section 1.3.2 and Table 1.1. Section 1.3.3 then summarizes the general requirements for the Inner Tracker. The dialogue continues into Section 1.4 where the Inner Tracker is overviewed in light of the HL-LHC stipulations (Sections 1.4.1 and 1.4.2). The detector design allows more developed deliberations of the radiation and track requirements (Sections 1.4.3 and 1.5.1 respectively). These are shown to be met in regards to the Strip portion of the ITk and its silicon sensors in particular in the following chapters.

\subsubsection{Structure of the Inner Detector}

The detector structure of ATLAS' current inner tracking system, the Inner Detector, is shown in Figure 1.6. Although not pictured the ID has the shelled cylindrical shape described above and seen in the ATLAS and ITk designs in Figures 1.5 and 1.7 respectively. The LHC's design targets of $1 \times 10^{34} \mathrm{~cm}^{-2} \mathrm{~s}^{-1}$ and $300 \mathrm{fb}^{-1}$ allowed the use of two detector technologies in the ID; gas phase and solid state. In the densest environments the use of silicon detectors with minute two dimensional (pixel) readout satisfied the requirements in granularity and radiation hardness, this sub-detector is shown in green in Figure 1.6. Further from the interaction point the tracking needs permit larger detector elements. Shown in blue is the ATLAS SemiConductor Tracker (SCT) a silicon micro-strip tracker which uses what are essentially elongated pixels (strips) to provide a rougher $2 \mathrm{D}$ coordinate. The SCT is the predecessor to the ITk Strip detector, it serves somewhat as a 'working prototype' for the ITk Strip detector upon which this work is based. Finally, occupying roughly half of the ID and with a comparatively rough granularity is the gas phase based Transition Radiation Tracker (TRT). It is shown in yellow in Figure 1.6. 


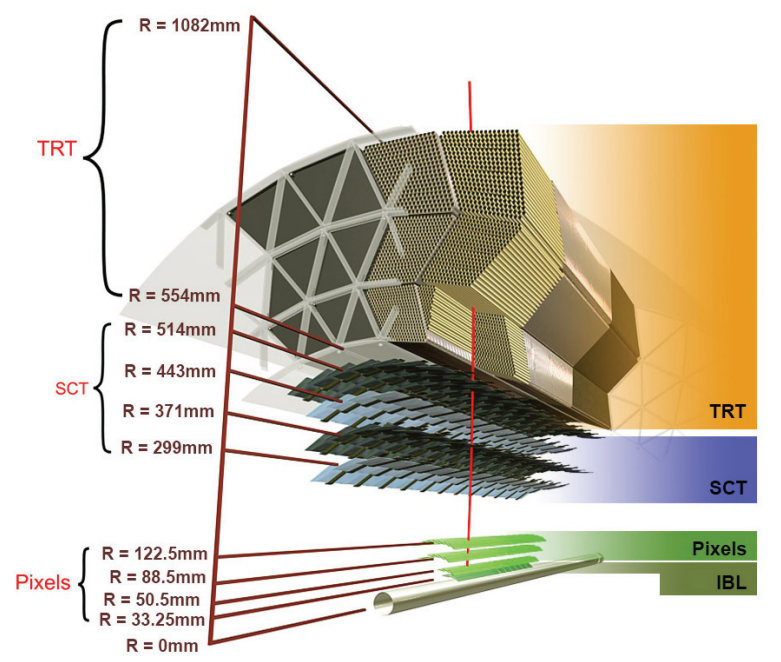

Figure 1.6: The detector structure of ATLAS' current inner tracking system, the Inner Dector (ID). The three detector sub-systems of silicon based Pixels and SemiConductor Tracker (SCT), and gas based Transition Radiation Tracker (TRT) are shown. The ID is cylindrical like seen in Figure 1.5. [9]

\subsubsection{Deficiencies in the Inner Detector}

Table 1.1 summarizes the design limits and current state of the ID, ATLAS, and LHC operation, with a focus on the parameters most relevant to the ITk. The HL-LHC and upgraded ATLAS requirements are given for comparison. Even without the base knowledge of the most fundamental quantities in the table (the luminosity targets) that the preceding sections have provided, just by considering Table 1.1 numerically, the need for the ID replacement is obvious. As mentioned in the section preamble, although coupled the main concerns for the inner detector can be grouped by their primary association with either the five to seven and a half increase in instantaneous luminosity, or, the order of magnitude increase in integrated luminosity. The summary given in Table 1.1 denotes this breakdown with horizontal lines (the operational period assumes its own category) [1-3].

The instantaneous luminosity related parameters have been discussed generally in Section 1.1. The experimental context offered in Section 1.3.1 allow the ID's unsuitability in this regard to be easily understood from Table 1.1. On the detector side the enormous increase in pileup will fatally compromise the tracking ability of the detector. The track and vertex-finding efficiencies, and background rejection are 


\begin{tabular}{|c|c|c|}
\hline Operational Parameter & $\begin{array}{l}\text { ID, LHC, and } \\
\text { ATLAS Design Limits }\end{array}$ & $\begin{array}{l}\text { HL-LHC Capabilities and Upgraded } \\
\text { ATLAS Requirements }\end{array}$ \\
\hline Experiment Lifetime & 10 years & $\begin{array}{l}\text { Current age: } ~ 7 \text { years } \\
\text { Age at upgrade: } 14 \text { years } \\
\text { Full duration: } 27 \text { years }\end{array}$ \\
\hline Peak Instantaneous Luminosity $\left(\times 10^{34} \mathrm{~cm}^{-2} \mathrm{~s}^{-1}\right)$ & 1.0 & $\begin{array}{l}\text { Nominal: } 5.0 \\
\text { Ultimate: } 7.5 \\
\quad \text { *with leveling }\end{array}$ \\
\hline $\begin{array}{c}\text { Pileup } \\
\text { (proton collisions per } 25 \text { ns bunch crossing) }\end{array}$ & $\begin{array}{c}\text { Design: } 23 \\
\text { Operational Max: } \sim 40 \text { (peak), } \sim 24 \text { (avg.) }\end{array}$ & $\begin{array}{l}\text { Nominal: } 140 \\
\text { Ultimate: } 200 \\
\quad{ }^{*} \text { with leveling }\end{array}$ \\
\hline ATLAS Trigger Rate $(\mathrm{kHz})$ & Level-1: 100 & $\begin{array}{l}\text { Single Mode; Level-0: } 1000 \\
\text { Dual Mode; Level-0: } 4000 \\
\text { Level-1 (new): } 400-600\end{array}$ \\
\hline Integrated Luminosity $\left(f b^{-l}\right)$ & $\begin{array}{c}\text { Pixels: } 400 \\
\text { SCT: } 700 \\
\text { Inserted Beam Layer: } 850\end{array}$ & $\begin{array}{l}\text { Nominal: } 3000 \\
\text { Ultimate: } 4000\end{array}$ \\
\hline $\begin{array}{l}\text { Maximum High Energy Particle Fluence } \\
\qquad\left(x 10^{15} 1 \mathrm{MeVn}_{\text {eqv }} \mathrm{cm}^{-2}\right)\end{array}$ & $\begin{array}{l}\text { Pixels: } 5.0 \\
\text { SCT: } 0.2\end{array}$ & $\begin{array}{l}\text { ITk Pixel: } 18.7 \\
\text { ITk Strip: } 1.2\end{array}$ \\
\hline Maximum Total Ionizing Dose $(M G y)$ & Pixels: 3.0 & $\begin{array}{l}\text { ITk Pixel: } 12.7 \\
\text { ITk Strip: } 0.5\end{array}$ \\
\hline
\end{tabular}

Table 1.1: Summary of current and future infrastructure operational parameters important to the ITk upgrade. Trigger rate will be discussed in the text of this Section (1.3.2). Particle fluence, its unit of $1 \mathrm{MeVn}_{\text {eqv }} \mathrm{cm}^{-2}$, and total ionizing dose will be discussed in context to the ITk in Section 1.4.3 and in context to the silicon sensors in Chapters 3, 4, and 5. [1-3] 
cogent examples of ID performance that will be negatively affected by the unacceptable increases in hit multiplicity. The magnitude of this issue is illuminated by the projection of $100 \%$ occupancy in the TRT at a pileup value of 200 .

Also associated with the instantaneous luminosity are required advances in the readout of the system. Triggering and data filtering must be performed at the extreme LHC speeds which dictate a pileup-laden bunch crossing every 25ns $(40 \mathrm{M} \mathrm{Hz})$. Interesting events are selected automatically using advanced algorithms that have been well trained over decades of collider experience. At the LHC the first level of data selection (Level-1) reduces the $1 \times 10^{34} \mathrm{~cm}^{-2} \mathrm{~s}^{-1}$ to a rate of $100 \mathrm{kHz}$. All readout systems were designed with this limit in sight and projections show that the data links between the inner detectors and their off-detector read-out receivers will shortly become saturated. As a result, above a pileup of roughly 70 proton-proton interactions, the vast majority of the silicon portion of the ID will not be accessible. The fundamental changes to the trigger system to cope with the collision rate (detailed below) also necessitate its replacement.

The main effect associated with the integrated luminosity (or total number of collisions) is that of radiation damage accumulation caused by the passage of years worth of high energy collision products. Every single component that enters the detector must meet stringent specifications on radiation hardness and reliability that are required to operate for extended durations with minimal intervention in the vicinity of the LHC's hostile interaction points. Each detector technology reacts differently to radiation but the effects are (almost $\left.{ }^{4}\right)$ always deleterious. The design limits in integrated luminosity, particle fluence (time integrated flux), and total ionizing dose all relate to the point at which the detector's basic functionality becomes unforgivably deteriorated by radiation damage.

\subsubsection{General Requirements for the ID Replacement}

The most basic, most general requirement for the ID's replacement (the ITk) is to maintain equal or better performance in HL-LHC conditions than the ID in LHC conditions. This directive includes the ultimate HL-LHC criteria of $7.5 \times 10^{34} \mathrm{~cm}^{-2} \mathrm{~s}^{-1}$, $4000 \mathrm{fb}^{-1}$, and a pileup of 200 [1]. In a more specific nature the requirements for the ITk are set by the ATLAS Phase-II physics goals and the need to conform to the ID envelope (with the adoption of some of its services). The preceding material is

\footnotetext{
${ }^{4}$ see Section 4.3.1
} 
preparation enough for the discussion found in this section. The radiation environment and tracking requirements are driven by the detector design and are discussed after the ITk is introduced in Section 1.4.

The layout of the detector must provide the full coverage (hermeticity) in each layer that is necessary for optimal tracking. It must do so for operation with multiple bunch crossing configurations (interaction region shapes). The resolution of the detector components (and their readout thresholds) must be chosen carefully to maintain acceptable levels of channel hit occupancy (channels with expected "hits" over total channels) and channel noise occupancy (hits due to statistical fluctuations) over the experiment lifetime. These parameters have one of the more dire effects on the tracking ability primarily through their influence on the track reconstruction pattern finding algorithm efficiency.

Secondary reactions with the detector material itself destroy the pure tracks which are the target of detection. They are also the source of a non-negligible portion of the damaging radiation in the detector. As such the hermeticity and occupancy concerns must be simultaneously wedded with a minimization of the material used (in a radiation length sense).

The large collision rate increase at the HL-LHC and its leveled nature will require special consideration of the readout system used therein. The challenge of quickly filtering through 8 billion collisions per second $(\mu=200)$ requires a thorough upgrade to the trigger system. The ID's replacement (the ITk) will have to adhere to the new ATLAS system which features the inclusion of track information and subsequently, track finding, at the hardware level. With the HL-LHC nominal and ultimate design targets being so different, a two scheme trigger system is projected. At instantaneous luminosities close to nominal the target is a $1 \mathrm{MHz}^{\mathrm{N}}$ "Level-0" hardware trigger with $6-10 \mu s$ on-detector latency (the latency and readout bandwidth must be able to accommodate the enormous HL-LHC data rate with less than $90 \%$ occupancy at any place or time). As the intensity climbs the need for hardware level track finding is a necessity required to reduce background levels. This will allow the blossoming Level-0 trigger (target $4 \mathrm{MHz}$ ) to be controlled to manageable levels with the introduction of a second hardware trigger level (Level-1 with a target of $400-600 \mathrm{kHz}$ ). As seen in Table 1.1 this will replace a single $100 \mathrm{kHz}$ trigger rate in the current LHC. [1]

Synergistic considerations for the ITk with respect to the ATLAS detector at large 
begin with the tracking-related matching of the "barrel" portion of the cylindrical detector with its muon tracking counterparts. Here barrel refers to the central portions of the ATLAS sub-detectors, this can be seen in Figures 1.5, 1.6, 1.7, and 1.8. The barrels have a different detection geometry than the end-caps which make up the top and bottom (or end) segments of the cylinders.

More mundane but just as essential are the need for the ID's replacement to conform to the existing envelope within the ATLAS machine. The cavity, magnet, routing and patching of services, as well as some of the cables will be re-used from the current detector. The radioactivity's effects on the silicon detector require operation at freezing temperature $\left(\sim-35^{\circ} \mathrm{C}\right)$ and the entire detector must also accommodate an air-tight environmental chamber with humidity control. The ITk cooling will be accomplished with bi-phase (evaporative) $\mathrm{CO}_{2}$ cooling using newly developed independent plants with $400 \mathrm{~kW}$ cooling capacity. The humidity envelope will join the Faraday cage, and neutron polymoderator (to avoid back scatter from the calorimeters) at the largest radii in the ITk system.

Other simple yet cogent considerations are the essential needs of being able to test the fully assembled detector prior to install (commissioning), installation of the detector itself, and its ability to stand unsupported for the experiment duration while maintaining the strict alignment requirements that tracking necessitates. Also, since the integrated luminosity is incomparably large, the radiation damage to the innermost layer(s) of the ITk obliges that they be replaceable (during a short sub-year length technical shutdown). Other than this, the nearly inaccessible ITk detector will operate essentially without physical intervention for the ten year lifetime. Extreme reliability in all components is therefore required.

The HL-LHC radiation fluence and dose projections found in Table 1.1 are developed further in Section 1.4.3 (wherein the concept of a $n_{\text {eqv }}$ will be explained). As mentioned the dose is closely related to the detector itself. It is for this reason that it and the tracking discussion (Section 1.5.1) have been postponed. A brief treatment of radiation hardness in regards to the ITk Strip detector operation is found in Section 2.7. More detailed studies in the context of the silicon sensors appear in Section 3.4 and Chapter 4.

A final, all-encompassing set of general requirements are associated with failure modes and safety. The system must be robust against more 'minor' failures that do not stop operation of the detector as a whole. Performance must be maintained 
in the event of uncorrelated (purely channel) and correlated (structural/componentrelated) malfunction of detection capability. The target in both of these cases is $10 \%$ detector-wide without degradation of the tracking performance discussed further in Section 1.5. Operation to the design limits is ensured by (a minimum) testing of all components to a safety factor of 1.5 (or 6 for components with batch variability). The system must also have fail-safes in place for more catastrophic failure events. These could include cooling excursions (in either direction), unexpected humidity increases, or power failure.

\subsection{The Inner Tracker}

The ATLAS Inner Tracker (ITk), shown in Figure 1.7, will be a fully solid state detector. This move was motivated by the increased luminosity targets of the HL-LHC (and the related effects discussed throughout this document). The more modern, silicon based detector technology offers advantages in resolution and radiation hardness in comparison to its less dense, gas-based counterparts. To satisfy granularity while maintaining minimal occupancy the ITk will utilize the semiconductor material in two finely tuned detecting geometries; microstrips and micropixels. These define the two sub-detector systems that make up the ITk; the ITk Strip and Pixel detectors. The silicon microstrip sensors that occupy the ITk Strip detector are the focus of this Master's thesis.

With its full silicon implementation the ITk and its sensors will face the unparalleled challenge of maintaining cost efficiency in the face of over $170 \mathrm{~m}^{2}$ of solid state tracking surface coverage, a factor of about three increase from the ID. This is one of the major design challenges for the ITk (especially for the much larger Strip portion of the detector). Coupling this with the next-generational goals in collider physics necessitate advancements in detector technology across the ITk's composite system. This document will focus on the fundamental progress in the ITk Strip detector's silicon sensors (Chapters 3, 4, and 5) with some minor discussion in respect to the more general ITk Strip detector system in Chapter 2.

\subsubsection{ITk Overview}

Figure 1.7 shows the Inner Tracker as it is rendered in its performance simulation framework. Figure 1.8 gives a schematic representation of the ITk layout. The Pixel 


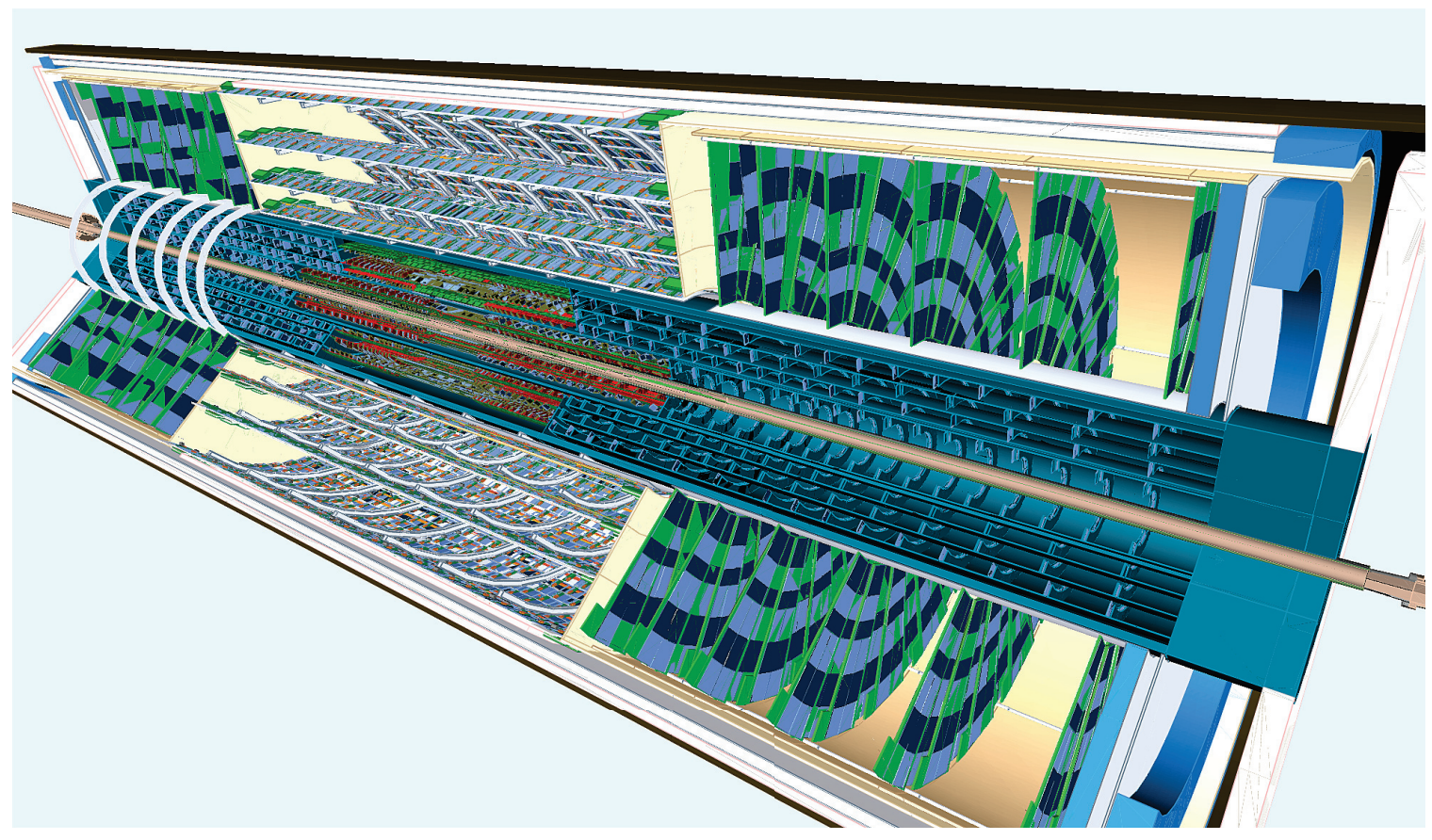

Figure 1.7: A visualization of the ITk as implemented in its performance evaluation simulation framework. [1]

and Strip sub-detectors are seen, as are their barrel and endcap sub-systems. The full ITk cylinder is roughly one meter in radius and six meters in length. It is technically an annular cylinder with the beam line occupying the annulus.

The cylinder closest to the beam pipe, constitutes the Pixel detector. It is the full $\sim 6 \mathrm{~m}$ length of the ITk and extends out to a radius of $\sim 30 \mathrm{~cm}$. It is shown in red (barrel) and dark teal (endcap) in Figure 1.7. In Figure 1.8 both Pixel sub-systems are shown in red.

The Strip detector fills the rest of the available ATLAS inner cavity volume. It is a more pronounced annular cylinder with length of $6 \mathrm{~m}$ and radial thickness of $\sim 60 \mathrm{~cm}$ (from radii $\sim 40 \mathrm{~cm}$ to the full $\sim 1 \mathrm{~m}$, a tracking lever arm consideration). It is shown in white/light blue (barrel) and green/blue (endcap) in Figure 1.7. In the layout figure (1.8), the Strip detector is shown in blue.

From the ITk layout, Figure 1.8, it can be seen that the defining characteristic of the barrel and endcap subsystems is the orientation of their detection components with respect to the interaction point. The use of inclined detectors in the elongated barrel of the Pixel detector is somewhat unconventional and is the namesake 


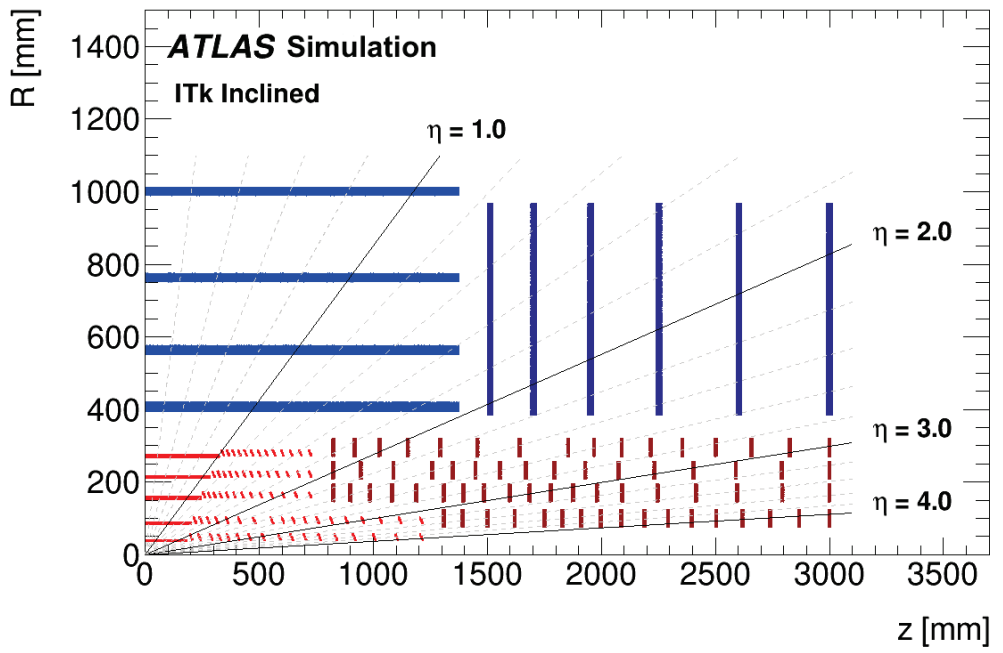

Figure 1.8: Simplified schematic layout of the ITk detector. Only active components and one quadrant of the symmetric detector is shown. $\mathrm{x}$-axis is along the beam pipe with the origin at the interaction point. $y$-axis is outwards in radius of the cylindrical ITk. "Inclined" refers to the layout; it is the front-runner of the two remaining designs. [1]

of the front-running layout design used in most results included in this document ("Inclined").

\subsubsection{ITk Layout}

The ITk layout shown in Figure 1.8 has been optimized through several iterations to meet the general, radiation and tracking requirements discussed in Sections 1.3.3, 1.4.3, and 1.5.1, respectively. An extensive simulation framework is used, the exhaustive nature of which can be appreciated by looking at Figure 1.7.

In the ATLAS detector coordinate system an important quantity is the pseudorapidity, $\eta$. It is defined as $\eta \equiv-\ln [\tan (\theta / 2)]$ [8], where $\theta$ is the polar angle in a spherical coordinate basis with $\mathrm{z}$ axis coinciding with the beam axis and the interaction point at the origin. Its significance is related to the beams' essentially head-on crossing. As a result the pseudorapidity assumes the role of key discriminating variable for many aspects of the ATLAS physics program (the foremost of which is background reduction).

For the ITk one innovation arising in its $R \& D$ phase has been the extension of tracking capability to a pseudorapidity of four $(|\eta|=4)$ (see Figure 1.8). This is a 
large increase from the current system's coverage limit of $|\eta|=2.7$. This advantage comes with large benefits for tracking and background rejection that are integral to the high luminosity operation. It is one of the main reasons why the ITk's performance projections actually exceed the basic requirement of meeting the ID's ability (see Section 1.5). This measure is achieved while still meeting all the general requirements in layout, granularity, readout, stability, and safety mentioned in Section 1.3.3.

\section{Material Considerations}

Another innovation in the ITk is a drastic reduction in the amount of material. The advantage is multi-fold; better tracking from less secondary interactions (especially for photons) and less radiation damage from the products of those secondary interactions.

A target value of less than one radiation length up to $|\eta|$ of 2.7 is obtained with a per layer value for the silicon sensors on the order of $2 \%$ of a radiation length. The inclusion of the support structure and services in the exhaustive simulation framework have allowed these targets to be addressed (as well as allowing the accurate dose calculations discussed in the next section).

Figure 1.9 summarizes the material budget considerations. In the top row a comparison of radiation length versus pseudorapidity for the ITk (right) and ID (left) shows the considerable strides made in the new detector. More careful measurements of modern prototype modules of the Strip detector are shown in the bottom row. The structure of these components is detailed in Chapter 2.

\subsubsection{Radiation Environment and Hardness in the ITk}

The HL-LHC's $O\left(1000 \mathrm{fb}^{-1}\right)$ integated luminosity has dire consequences for the atomic structures that sit in the vicinity of its billions of $13-14 \mathrm{TeV}$ proton-proton collisions per second. Radiation damage is one of the foremost concerns for the ITk and similar inner tracking systems. All components must be rad hard; operable with minimal degeneration up to and exceeding (1.5 safety factor) the HL-LHC design limits.

The ITk simulation framework has allowed the calculation of particle fluxes as a function of position in the detector. These important results, obtained through the FLUKA integrated Monte Carlo software package, are summarized in Figures 1.10 and 1.11, and Table 1.2. These show the effects of different particles at different 

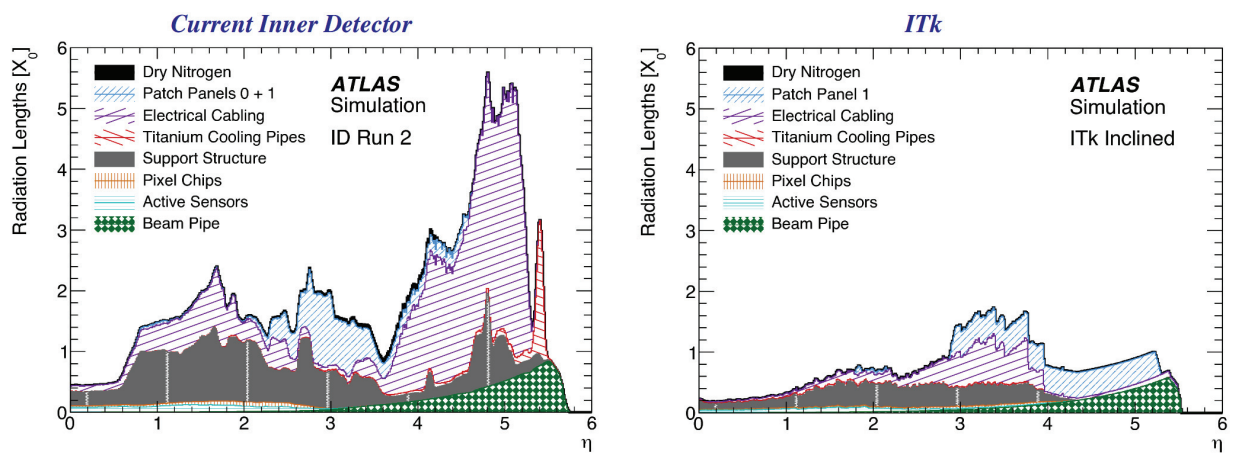

Short Strip Barrel Module
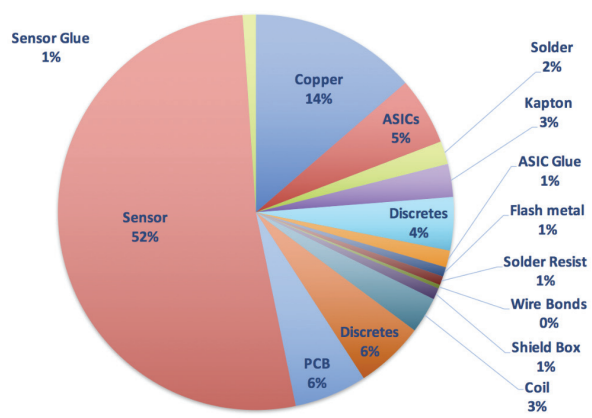

Long Strip Barrel Module

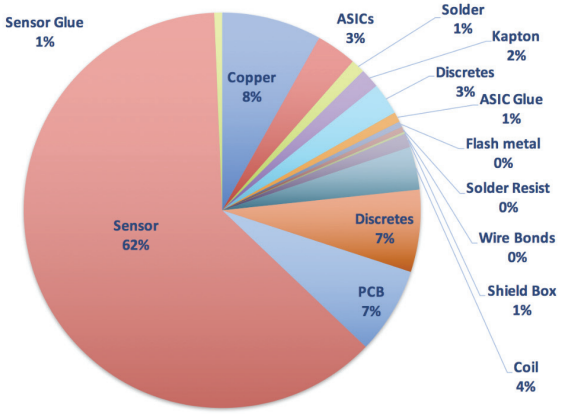

Figure 1.9: Material reduction summary. Top; radiation length as a function of $\eta$ for the current ATLAS detector (Top Left) and the ITk (Top Right). The contributions from each type of material are stacked. Bottom; fractional radiation length contributions at the ITk Strip module level, for inner barrel (Bottom Left) and outer barrel (Bottom Right) modules (see Section 2.4 for module description). [1] 


\begin{tabular}{llll} 
Layer & $\begin{array}{l}\text { Radius } \\
{[\mathrm{mm}]}\end{array}$ & $\begin{array}{l}\text { Maximal Fluence } \\
{\left[\mathbf{n}_{\text {eq }} / \mathbf{c m}^{2}\right]}\end{array}$ & $\begin{array}{l}\text { Maximal Dose } \\
{[\text { MRad] }}\end{array}$ \\
\hline Strips & & & \\
\hline Long Strips & 762 & $3.8 \times 10^{14}$ & 9.8 \\
Short Strips & 405 & $7.2 \times 10^{14}$ & 32.5 \\
End-cap & 385 & $1.2 \times 10^{15}$ & 50.4 \\
\hline Pixels & & \\
\hline Layer 0 & 39 & $1.87 \times 10^{16}$ & 1268 \\
Layer 1 & 75 & $0.59 \times 10^{16}$ & 549 \\
Layer 2 & 155 & $0.22 \times 10^{16}$ & 129 \\
Layer 3 & 213 & $0.15 \times 10^{16}$ & 87 \\
Layer 4 & 271 & $0.11 \times 10^{16}$ & 53 \\
End-cap & 80 & $0.62 \times 10^{16}$ & 477 \\
\hline
\end{tabular}

Table 1.2: Maximal fluence and dose expectations at $4500 \mathrm{fb}^{-1}$. [1]

fluences and positions (refer Figure 1.8) offers a complete picture of the radiation situation that is so integral to the case of the HL-LHC, ITk, and this work.

The calculation of total particle fluences is facilitated for the variant ITk environment by the Non-Ionizing Energy Loss (NIEL) hypothesis. This hinges on the experimental findings that the dominant effects of radiation damage (discussed in Section 3.4) trend (essentially) proportionally with the NIEL of a passing particle and not its identity. The result is the ability to compare the effects of irradiation from different particles using their equivalent radiation fluences. The conventional unit is the $1 \mathrm{MeV}$ neutron equivalent, $n_{\text {eqv }}$. This amount of damage can be put into context of the later discussion on silicon sensors (Chapters 3 through 5) when considering that $1 n_{\text {eqv }}$ is predicted to produce a lattice-displaced silicon with enough energy to create a $100 \mathrm{~nm}$ track of defects (see Section 3.4.1) [23].

As the title of this document foreshadows a large portion of the ITk's and this work focuses on the radiation hardness of the new silicon sensors. This is because they are the backdrop for the fundamental physics processes that allow the ITk its tracking nature. In this sense they are the most sensitive component in the most radioactive environment in ATLAS. As was seen in Table 1.1, a sizable advance in radiation hardness is needed moving into the HL-LHC phase. While a brief discussion of the integrated Strip detector end-of-lifetime performance is given in Section 2.7 the heart of the rad hard discussion is towards the end of Chapter 3 and its follow-up 

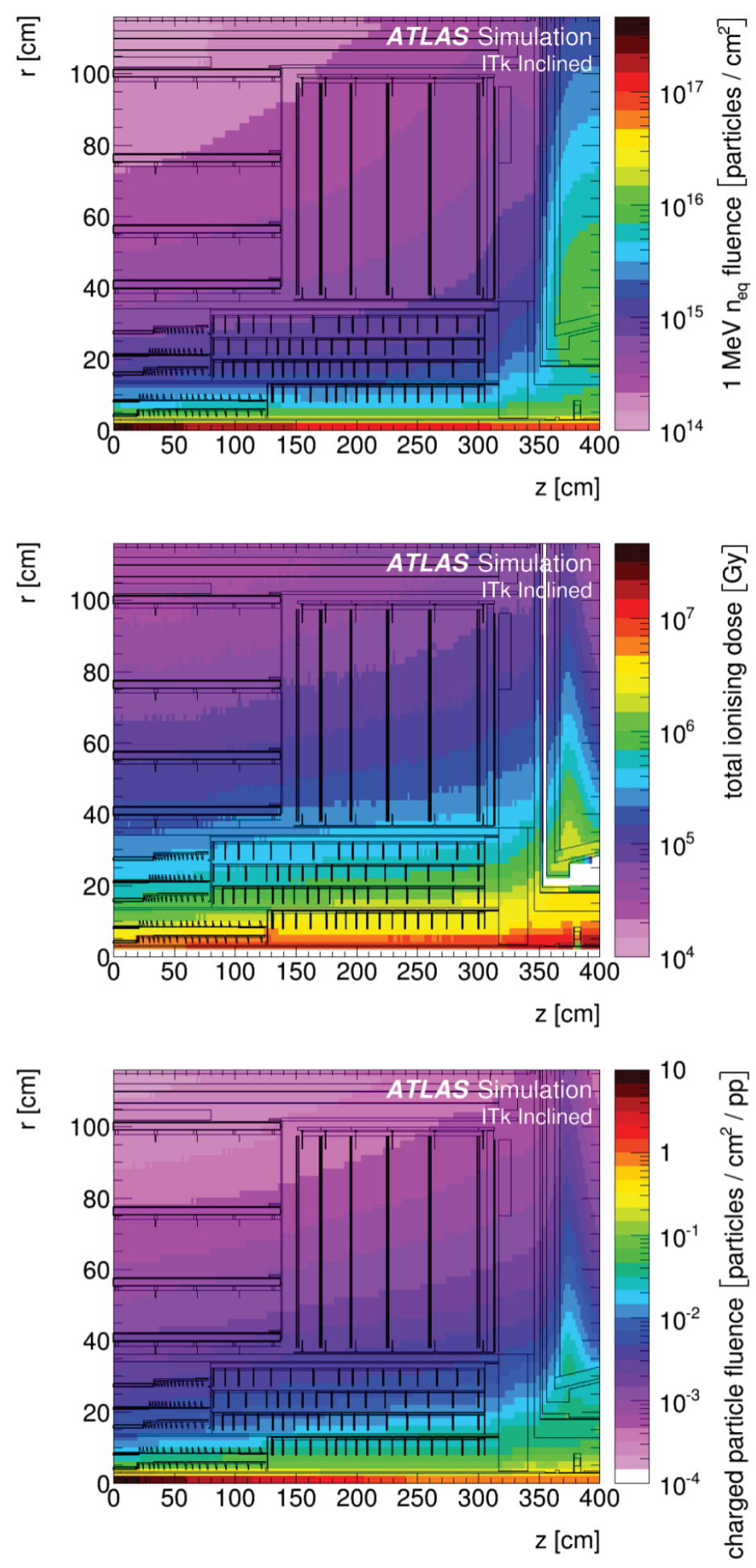

Figure 1.10: Fluence and dose distributions expected in the ITk at $3000 \mathrm{fb}^{-1}$. Top; the $1 \mathrm{MeV} n_{\text {eqv }} \mathrm{cm}^{-2}$ NIEL fluence. Centre; total ionizing dose (TID). Bottom; charged particle fluence per proton-proton collision in the detector. [1] 


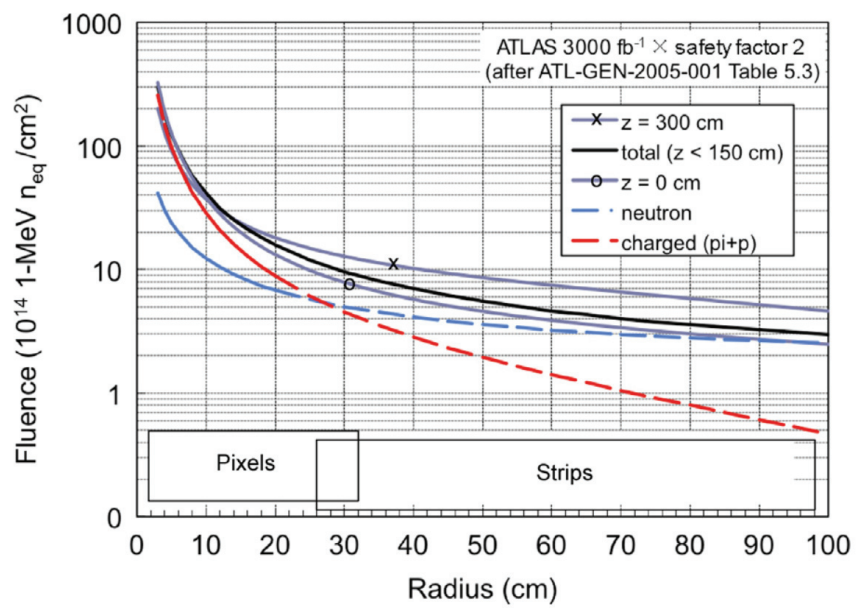

Figure 1.11: Charged particle, neutron, and total fluences as a function of radius at $6000 \mathrm{fb}^{-1}$. For the total fluence several beam axis (z) positions are shown (refer Figure 1.8). [10]

throughout Chapter 4.

The main consequences of irradiation in the ITk Strip's sensors are the rise in operational high voltage (HV) current and the reduction in collected signal. The latter of these can lead to inefficient track reconstruction since readout thresholds must be adjusted closer to the Gaussian noise profile (occupancy concerns). Mitigation is only minimally possible by raising the operational $\mathrm{HV}$, a route that is encouraged against by the rising currents and the high voltage's predominant role in detector power consumption. While these concerns are primarily associated with the NIEL damage, ionizing interactions are also problematic causing issues in the channel isolation and readout architecture (see Figure 2.10 for the latter).

The order of magnitude increase in the total number of high-energy collisions results in a similar increase in the total fluences and ionizing doses that the inner detector will experience. As will be seen, this move into the $10^{16} n_{\text {eqv }} \mathrm{cm}^{-2}$ range leads to an entirely new realm of semiconductor physics wherein a previously undiscovered beneficial phenomena (Space Charge Limited Avalanche, SCLA) provides the key to successful operation (Section 4.3.1). This and other necessary detector innovations are required to overcome the debilitating damages caused. The world-leading R\&D campaign for the sensors is detailed in Chapter 4. The current iteration of Strip sensor prototypes are part of this noble heritage lending weight to the torch-bearing results presented in this Master's thesis (Sections 5.3 and 5.5). 


\subsection{Expected Performance of the ITk}

This chapter is concluded with a look at the tracking functionality of the ITk and the physics gains offered by it and the HL-LHC's statistical increase.

The tracking prerequisites for the ITk detector are given by the physics goals defined in the ATLAS HL-LHC doctrine. Living up to its general purpose nature, both of these are extremely varied and complex. Only an exceedingly cursory treatment is attempted in this section. The basic ITk stipulation of equal performance to the current infrastructure in HL-LHC conditions is developed further in Section 1.5.1. It should be noted that the tracking ability described herein must adhere to the general requirements overviewed in Section 1.3.2. The ITk's simulated design implementation as described in the preceding text is applied in advanced collision simulations. More than adequate performance is obtained, the slightest jist of which is addressed in Section 1.5.2. Finally, a specific example of one of the multitude of physics goals that were discussed in a more idealistic sense at the start of this document wraps up the largest scale examination, in Section 1.5.3.

\subsubsection{Tracking Requirements}

As the "Inner Tracker" the most fundamental measure of ability is in track reconstruction. Table 1.3 shows the required efficiency for some of the most numerous particles that will traverse the detector volume. The role of the leptonic (non-strongly interactive), heavy, high energy muon as a minimum ionizing particle (mip) is reiterated here in its strict reconstruction restraints. This nature will be fully understandable after the discussion on semiconductor tracking physics given in Chapter 3.

In general the tracking capability must allow for coverage of the full interaction region, as well as an optimized, uniform response in the sensitive transverse direction. It can be seen that the novel tracking extension from $|\eta|$ of 2.7 to 4 in the ITk is absent from Table 1.3. Most tracking parameters in this newly exploited area are relaxed because of the weaker magnetic field and higher particle fluences associated with the (very) forward regions.

The overlay of up to 200 background (pileup) hard scatter events over the interaction products of interest requires high detector granularity. This resolution consideration is integral to operation in the HL-LHC atmosphere. Occupancy is the best 


\begin{tabular}{lccc} 
Particle & $p_{T}$ range & \multicolumn{2}{c}{ Required Efficiency } \\
& & $|\eta| \leq 1.0$ & $1.0<|\eta|<2.7$ \\
\hline Muon & $p_{T}>3 \mathrm{GeV}$ & $>99 \%$ & $>99 \%$ \\
Pion & $p_{T}>1 \mathrm{GeV}$ & $>90 \%$ & $>85 \%$ \\
Electron & $p_{T}>5 \mathrm{GeV}$ & $>90 \%$ & $>85 \%$ \\
\hline
\end{tabular}

Table 1.3: Basic tracking performance targets for the ITk. Track finding efficiencies for the particles with the most significant physics impact. [1]

measure of the detector's ability to cope with the channel saturation that is detrimental to the pattern recognition algorithms that drive the tracking performance. In the ITk, the target of 1\% average occupancy and $90 \%$ event maximum have been chosen to acquiesce to the demands of track separation, vertex reconstruction, vertex association, particle identification, background discrimination, readout bandwidth, and trigger rate. The rate of fake tracks in the detector is likewise limited to values of $1 \%$ detector-wide average, and 3\% locally. [1]

\subsubsection{Tracking Performance}

The results found in this section are the result of a full simulation with the GEANT4 radiation tracking, Monte Carlo based, simulation toolkit in the context of the ITk framework that can be seen in Figure 1.7. The reconstruction software is adapted from that used in the current ATLAS system and although not yet fully optimized is already in a state compliant with the tracking goals laid out in the previous section(s).

Tracks are formed from patterns in the three dimensional 'point' measurements obtained in each silicon layer. These are formed from the centroid positions of clusters of activated detection elements ("hits"). The basic method by which an individual 3 -D measurement is made is easily envisioned for the pixels and is developed in the context of the Strip detector in Section 2.3 and more fundamentally in the context of the silicon sensors in Section 3.3.

Groups of three hits congruent with a particle coming from the interaction region are used as "seeds" in the first, combinatorics-based phase of track reconstruction. Higher purity strip layer seeds are weighted preferentially in an efficient pattern recognition algorithm which builds the larger track segments. The ambiguities in hit association are resolved with an iterative procedure implementing a scoring metric based on the presence or absence of hits. The resulting track candidates are subject 


\begin{tabular}{ccc} 
Requirement & \multicolumn{2}{c}{ Pseudorapidity interval } \\
& $|\eta|<2.7$ & $2.7<|\eta|<4.0$ \\
\hline Pixel+Strip clusters & $\geq 9$ & $\geq 9$ \\
Pixel clusters & $\geq 1$ & $\geq 1$ \\
Holes & $<3$ & $<3$ \\
Pixel holes & $<2$ & $<2$ \\
Strip holes & $<3$ & $<3$ \\
Double Strip holes & $<1$ & \\
$p_{T}[\mathrm{MeV}]$ & $>900$ & $>400$ \\
$\left|d_{0}\right|$ & $\leq 2 \mathrm{~mm}$ & $\leq 10 \mathrm{~mm}$ \\
$\left|z_{0}\right|$ & $\leq 25 \mathrm{~cm}$ & $\leq 25 \mathrm{~cm}$
\end{tabular}

Table 1.4: Track reconstruction requirements for the two main psuedorapidity regions of the ITk. Requirements shown are for a "reconstructed" track in the simulation. [1]

to a final set of kinematic and vertex cuts that further reduce the less interesting background events.

Table 1.4 summarizes the track reconstruction parameters in the two main pseudorapidity regions of the detector. The simulation has shown that the best high density efficiency results from ensuring that the tracks have high hit counts and low "hole" counts (where hole refers to the lack of a hit in the silicon layers along the track path). These parameters are key to achieving the target $99 \%$ for the duration of the HL-LHC lifetime. As Figure 1.12 shows they are easily met with the ITk layout given in Figure 1.8 for a mip with a high momentum in the plane transverse to the beam axis.

Track reconstruction efficiency is associated with fake tracks created in the pattern recognition software. These result from random associations of hit clusters of different "truth" particles being attributed to the same (incorrectly) "reconstructed" particle. Efficiency is evaluated in simulation using truth particle tracks. These are obtained from the primary decay products of the collisions with stricter kinematic and vertex constraints on the tracks than is given in Table 1.4. Requiring that at least $50 \%$ of the clusters on a given reconstructed track correspond to a single truth particle defines the criterion for a good (matched) track. The track reconstruction efficiency then becomes the ratio of good (matched) reconstructed tracks to the truth level tracks. 


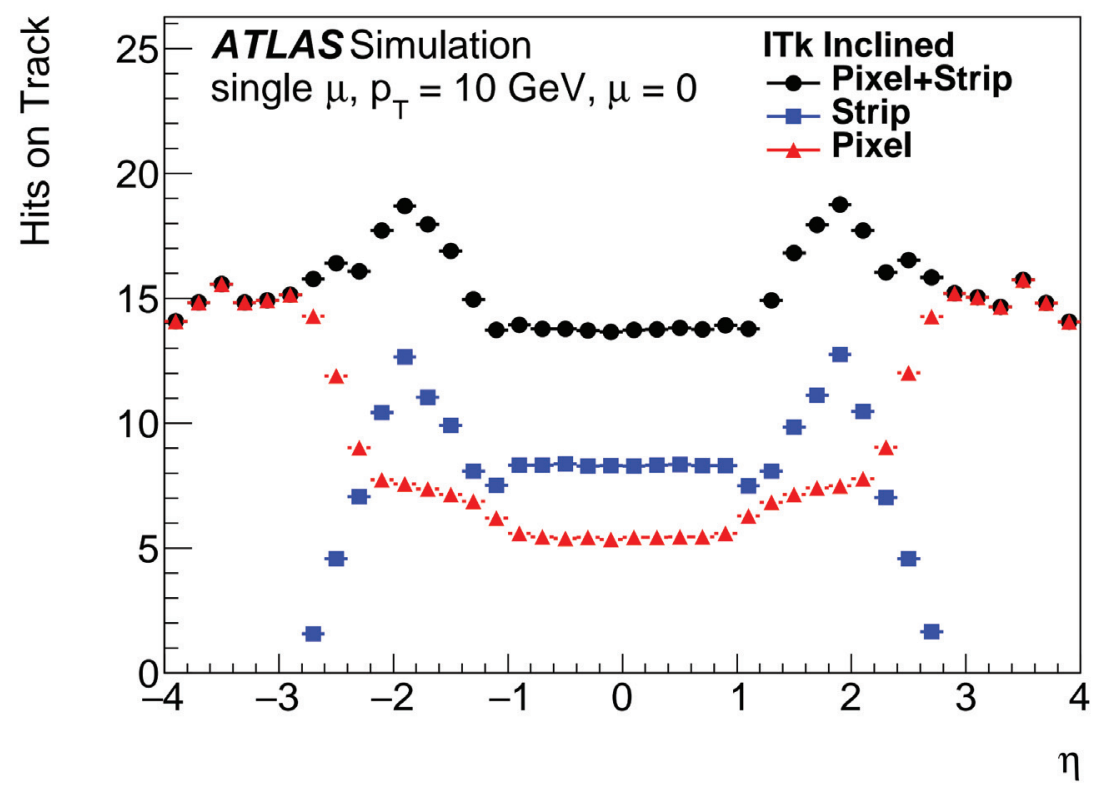

Figure 1.12: Evidence that track reconstruction efficiency demands will be met in the ITk. Simulation results showing predicted hits per track counts as a function of psuedorapidity. Both detector contributions are given. A muon with $10 \mathrm{GeV}$ transverse momentum is simulated, a taxing signature to detect with significant physics impact. [1] 
The granularity of the detector elements needs to be small enough to attain the necessary track resolution for the pattern recognition to work. Figure 1.13 shows that the goal of $1 \%$ average and $3 \%$ local maximum mentioned in the previous section is met. The Strip detector is detailed in the centre and right plots. The trends seen can be understood from the descriptions of the detector and sensor structures in Chapter 2 and Section 4.1.2.

It has been found that the contributions from the detector noise (sensor dominated spontaneous current fluctuations resulting in an unexpected hit) to the pattern recognition degradation are minimal if the signal to noise ratio can be kept above $10: 1$ for the duration of the experiment. This fundamental detector requirement is evaluated in a recent iteration of fully irradiated ITk Strip prototype module (a small integrated readout-sensor component). These results, obtained within the scope of my Master's research are discussed in Section 2.4. They show that the bold promises offered in this section will be achieved.

The design of the Inner Tracking detector and its reconstruction software are optimized together to accomplish the many technical tracking goals of the ATLAS HL-LHC physics program. Figure 1.14 shows that the track reconstruction efficiencies and fake rates obtained meet the stipulated values laid out in Table 1.3 and the accompanying text. In the left plot the basic requirement of meeting or exceeding the ID performance is shown to be the latter in the most basic requirement of track reconstruction of minimum ionizing particles. In the right plot of Figure 1.14 the fraction of reconstructed tracks to produced particles is shown as a function of number of proton-proton collisions with near unity results for the central psuedorapidity range.

These advanced simulation results provide strong evidence that the ITk have exemplary tracking capability even in the extreme HL-LHC conditions of 200 pileup collisions per bunch crossing. Here the discussion has been restricted to the vital tracking reconstruction efficiency parameter. Given in the ITk Strip Technical Design Report [1] are projections of the ITk's performance in regards to tracking resolution quantities, system redundancy, vertexing, and for physics related goals in reconstruction of the main decay products. These likewise support the prime directive of equal or better performance than the ID. Again the lean is towards the latter an accomplishment gained by the tracking extension, material reduction, and experience (from the SCT assembly and operation) that are implemented in the ITk. 

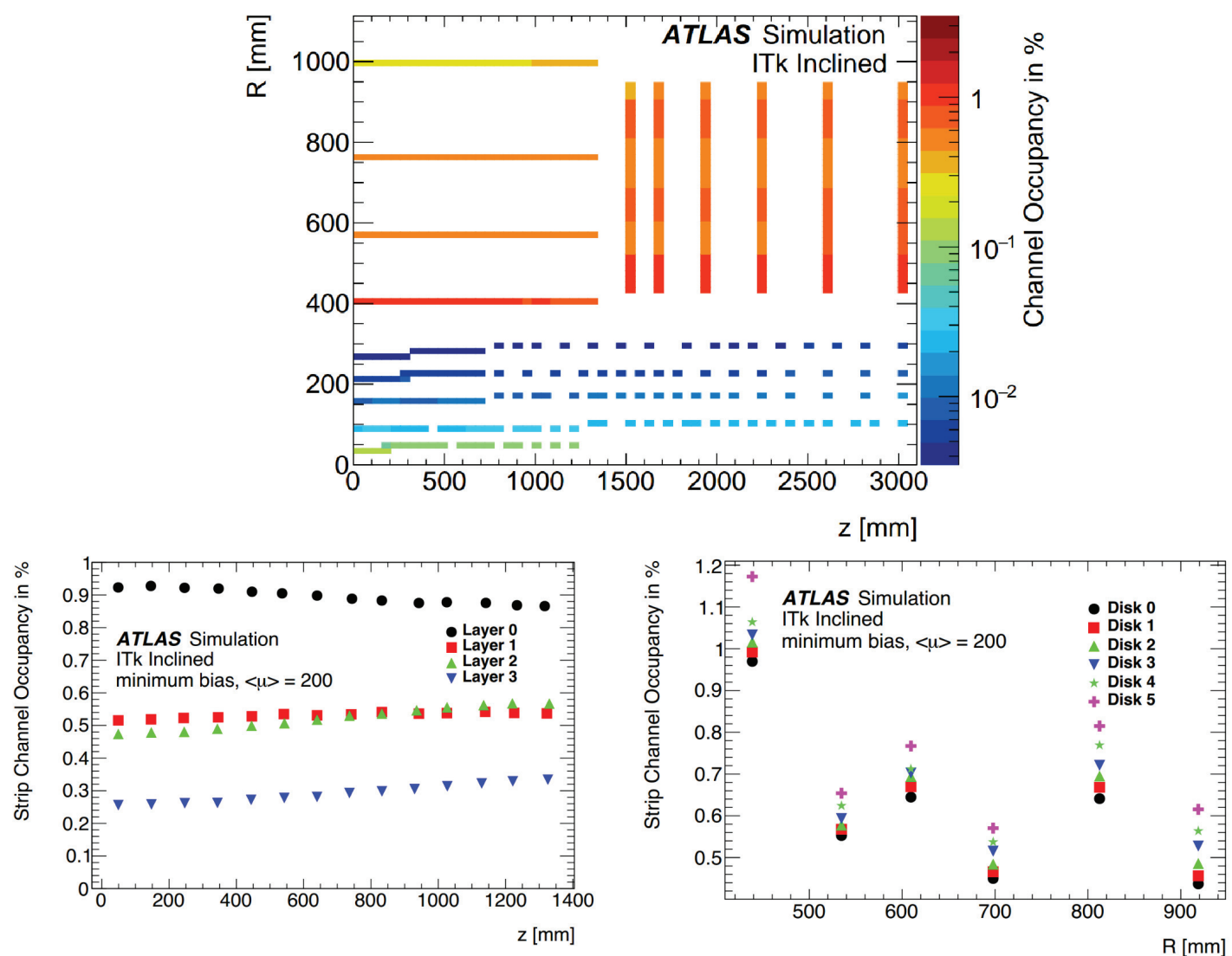

Figure 1.13: Occupancy predictions in the ITk and ITk Strip detectors at $3000 \mathrm{fb}^{-1}$ and $\mu=200$. Top; average channel occupancy over the full ITk layout (refer Figure 1.8). Bottom; more detailed views of average channel occupancy for the Strip detector's barrel layers (left) and endcap disks (right). [1] 

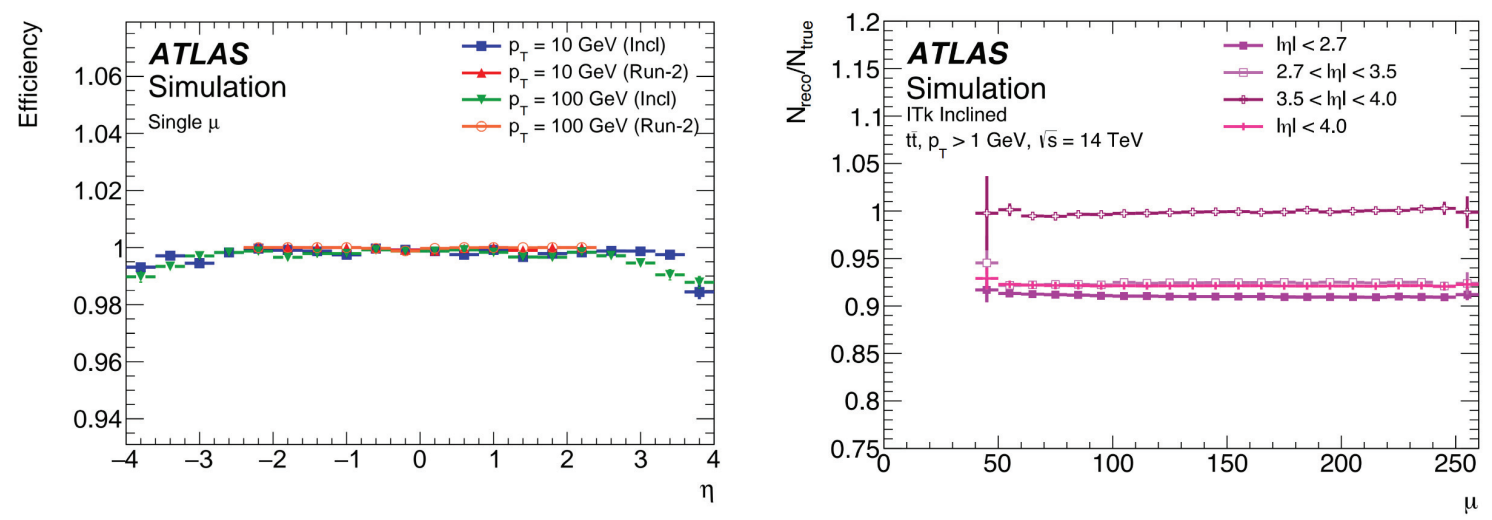

Figure 1.14: Track reconstruction efficiency projections for the ITk. Left; as a function of psuedorapidity for (mip) muons with 10 or $100 \mathrm{GeV}$ transverse momentum. Run 2 results for the ID are shown in red and orange with the ITk in blue and green. Right; as a function of pileup for the main pseudorapidity regions of the ITk. [1]

\subsubsection{Higgs Physics Benchmark}

At the beginning of this chapter we saw some of the questions that the ITk and HL-LHC will be built to answer (Section 1.1). The physics reach of the HL-LHC is significantly augmented in comparison to its current state. As seen, the nature of this augmentation in regards to the detection of new (and existing) physics is the advantage of statistics. Given in this section a very brief glimpse of the consequences of the high luminosity and Inner Tracker upgrades on humanity's most basic understanding of the universe. The results of the full simulations like the one mentioned in the preceding sections are parametrized to allow the impact of the ITk (and ATLAS) tracking in HL-LHC conditions on physics analyses to be ascertained.

Fittingly, the focus is on the Higgs boson; conveyor of mass, keystone of the standard model, and star discovery at the LHC, so far. An overview of the theory of the mass mechanism is given which provides the specific motivation for LHC-leading field of Higgs physics. This is followed by a quick discussion of the statistical effects of the HL-LHC upgrade using two examples. The more general across-the-board reduction in the decay width and coupling uncertainties, and the more ITk related, gains in the rare Higgs to dimuon decay channel. 


\section{The Higgs Mechanism}

The significance of the Higgs boson to the Standard Model (our current understanding of the realm of fundamental particles) and (thus, understandably,) to the universe at large is singular; it is responsible for particle mass.

The mechanism by which particles gain mass is called spontaneous electroweak symmetry breaking (EWSB). The gauge nature of the Standard Model theory (see Section 1.1.1) dictates massless force carriers; (the gauge field, spin-1 bosons ${ }^{5}$ ). However, those moderating the weak force are decidedly massive. This is understood as a symptom of what is called the Higgs mechanism.

The Higgs mechanism sees the two (physical) degrees of freedom exhibited by the massless force carriers (understandable most simply perhaps by the right and left handed polarization states of a photon) added by the third longitudinal degree exhibited by particles with mass. This extra degree of freedom can only result if the weak force bosons couple directly (strongly) to a different type of particle with only the missing longitudinal degree of freedom. These are the spin-0, scalar bosons.

In quantum field theory these scalar bosons are the result of the spontaneous breaking of gauge symmetries. Cases when the gauge field obtains a non-zero value across space (like the global field in a magnet but in four dimensions). The lowest energy configuration of the field potential in this case is that of the "mexican hat", shown for two complex dimensions in Figure 1.15 (as compared to a flat zero field for a massless particle). The lowest point in the potential spectrum constitutes the vacuum expectation value ("vev") for the field excitation (particle). The number of scalar bosons associated with the field is related to the symmetry (in the sense of the Lie algebra groups) that is broken. [11]

As measurements were being made at higher and higher energies the kinship between the electromagnetic and weak forces was becoming palpable. The 1960s proved to be the critical point when the theoretical understanding had matured enough to realize that they were actually phenomena related to each other and the mechanism for mass. The two symmetries once constituted a larger (electroweak) symmetry group that underwent spontaneous symmetry breaking as the energies in the cooling

\footnotetext{
${ }^{5}$ Bosonic (force carrying) and fermionic (matter) particles are the two main classifications of fundamental particle type in the Standard Model. They are distinguished technically by their (quantum) spin; integer and half integer, respectively, which also allows composite particles to be classified.
} 


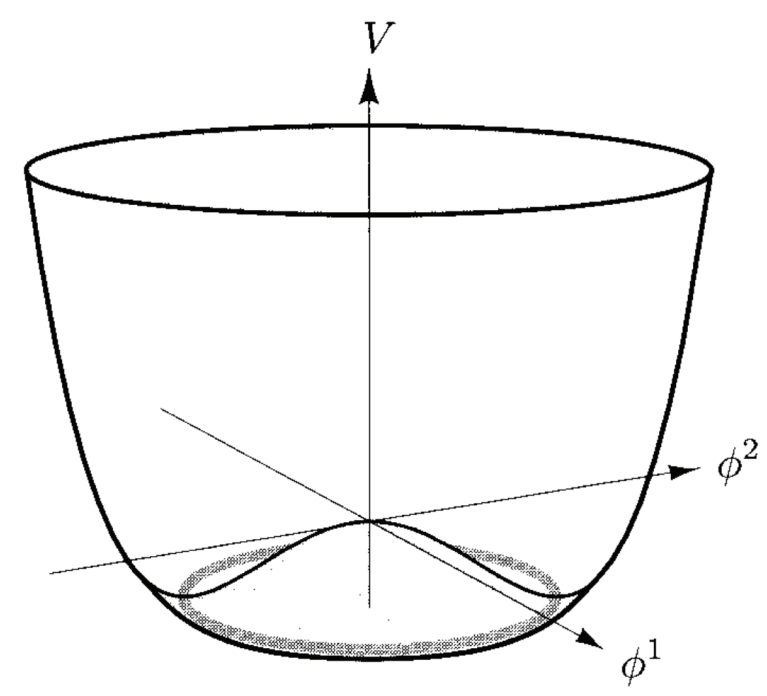

Figure 1.15: The "mexican hat" potential (V). The $\phi^{1}$ and $\phi^{2}$ axes correspond to the complex symmetry axes. Since the electroweak symmetry group is SU(2) this potential defines the minimum energy configuration of the Higgs field. [11]

universe allowed the evolution of a non-zero vev in the scalar Higgs field. The three weak force carrying bosons acquiring mass through the Higgs mechanism. ${ }^{6}$ The fourth scalar boson produced from the breaking of the $\mathrm{SU}(2)$ electroweak symmetry remains (yielding massless photons) and acquires mass due to its non-zero vev, the Higgs boson. All particles then acquire mass as they couple to this pervasive excitation that is the Higgs boson field (with their mass defined by the coupling strength).

\section{Higgs Physics Gains at the HL-LHC}

So far all measurements of the $125 \mathrm{GeV}$ Higgs boson discovered in 2012 corroborate this Standard Model understanding. The significance of precision measurement of the Higgs boson parameters to new physics is now understandable and is rooted in its scalar nature. Analyses of the $125 \mathrm{GeV}$ Higgs' couplings and decay lifetime offers one of the very best 'known' leads to undiscovered phenomena.

The effect of decreasing statistical uncertainty that is obtained with the HL-LHC upgrade is summarized for these important quantities in Figure 1.16. The relative uncertainty on analyzed signals are shown. On the left side the relative uncertainty

\footnotetext{
${ }^{6}$ The massless electroweak mediators "eating" the scalar bosons. Where eating is the technical term for the strong coupling that results in the indistinguishably point-like massive weak bosons. [11]
} 


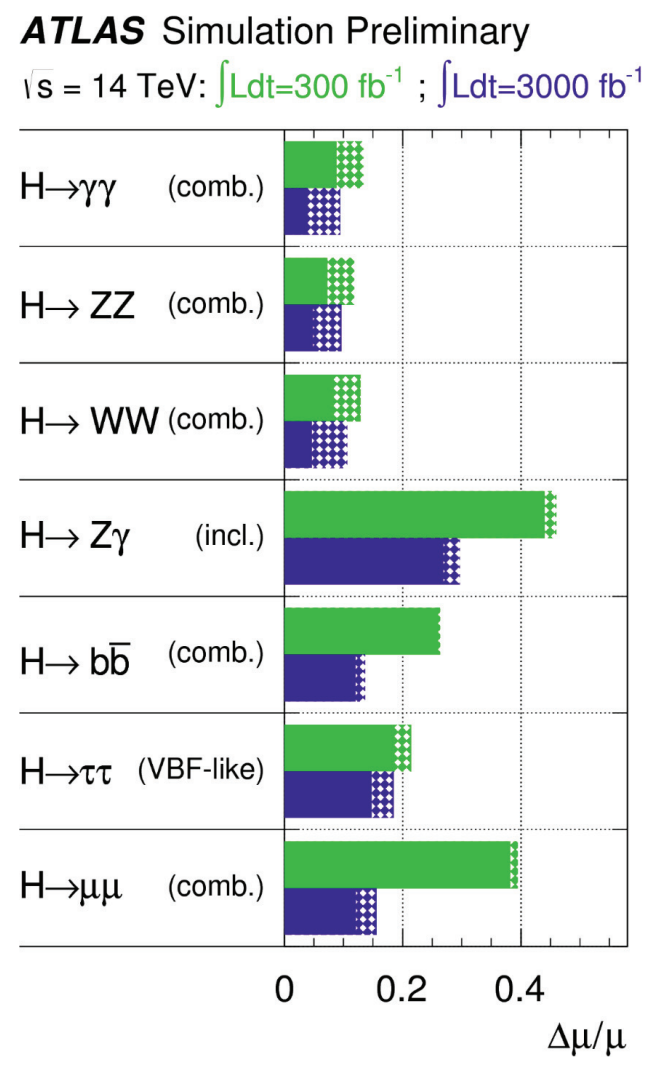

ATLAS Simulation Preliminary Is = $14 \mathrm{TeV}: \int L d t=300 \mathrm{fb}^{-1} ; \int L d t=3000 \mathrm{fb}^{-1}$

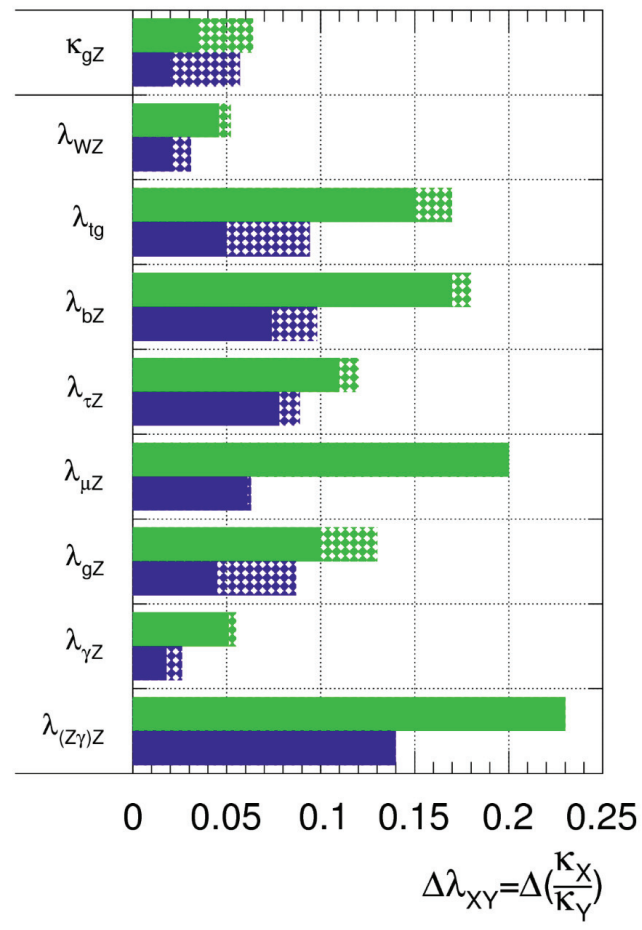

Figure 1.16: Example of the reduction in (relative) statistical uncertainty that will be obtained at the LHC. Left; Higgs boson decay widths (refer to Figure 1.2) with the production mechanism contributers shown in brackets. Right; coupling scale factor ratios. A $125 \mathrm{GeV}$ Standard Model Higgs boson is assumed. [12]

on the signal strength, $\eta$, is given for a variety of the Higgs decay channels shown in the right plot of Figure 1.2. Integrated luminosities of $300 \mathrm{fb}^{-1}$ and $3000 \mathrm{fb}^{-1}$ are shown in green and blue respectively. The right plot shows a similar result for the coupling, $\kappa$, related properties. In the parametric simulation results of Figure 1.16 a $125 \mathrm{GeV}$ Standard Model Higgs and $14 \mathrm{TeV}$ data are assumed.

A strong benchmark for the ITk tracking performance at HL-LHC instantaneous and integrated luminosity targets is the rare process $H \rightarrow \mu \mu$. The cross-section for this decay is so small that it is not even shown on the plot in the opening sections (Figure 1.2 right). It is of specific interest since it exhibits the direct coupling of the Higgs boson to the heavy-electron-like muons, the only detectable fundamental particle of that type. ${ }^{7}$ This is an especially taxing analysis for the ITk tracking

\footnotetext{
${ }^{7}$ Second generation lepton.
} 

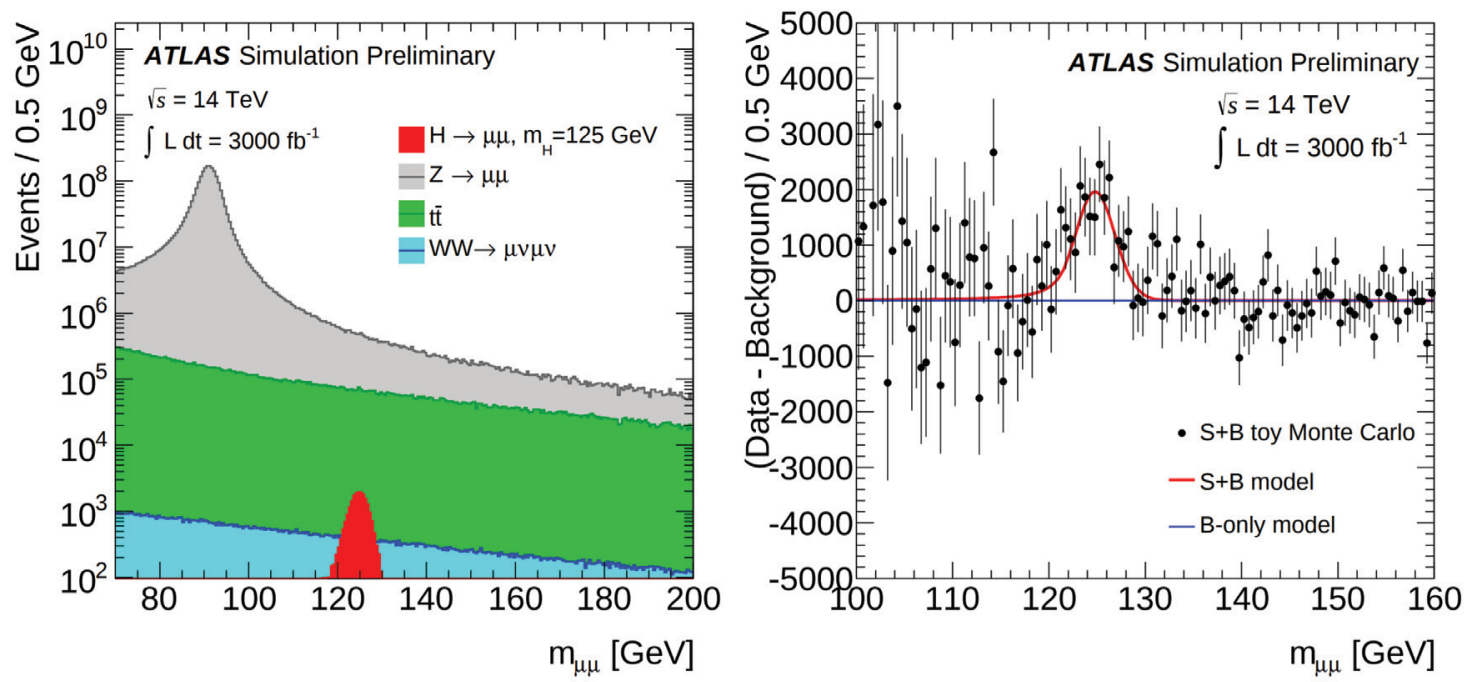

Figure 1.17: HL-LHC gains in the $H \rightarrow \mu \mu$ channel. Left; signal (red) overlaid on stacked backgrounds. Right; background subtracted result, a signal significance of seven sigma is obtained. [13]

capability relying on tight reconstruction of the muon energies and momenta to negate the significant background contributions in the signal region.

Figure 1.17 shows the simulated results at $3000 \mathrm{fb}^{-1}$ of $14 \mathrm{TeV}$ data for the $H \rightarrow \mu \mu$ analysis. On the left is shown the signal magnitude in red overlaid over a stacked plot of the dominating backgrounds shown in grey, green, and blue. It is a log scale. The peak in the grey signal is due to the $\mathrm{Z}$ boson (its mass is $\sim 91 \mathrm{GeV}$ ). The right plot shows the background subtracted "money plot" projection for the channel with a Monte Carlo sample. A signal significance of seven sigma is obtained [13]. The modeled distribution is shown in red and blue. The x-axis on these plots (Figure 1.17) is the invariant mass (read total energy; includes kinematic energy and rest mass) of the two muons. In other words it is the mass of the particle that has produced the two muons, the Higgs boson.

The event selection in this analysis (Figure 1.17) is optimized (as per usual) for high signal efficiency coupled with high background suppression efficiency. Event classification algorithms split the data into carefully chosen subsets to increase the signal to background separation sensitivity. Maximum likelihood fits are applied. This process is such that the resolution of the muons' energies and momentum (their invariant mass) dominants the signal separation. The ITk's track contributions are an absolute necessity in this matter. 


\section{Chapter 2}

\section{The ITk Strip Detector}

This chapter begins the collimation of focus towards the main topic of this thesis; the ITk Strip silicon sensors. These sensors are the basic building blocks of the Strip detector providing the essential mechanism for the tracking capacity in the majority of the ITk volume (as described in Section 1.4). However, before they are intimately discussed in Chapters 3, 4, and 5 they must be placed in the context of the larger experiment structures. Here, the missing link between the discussion of the fundamentally motivated ITk as a whole, presented in Chapter 1, and this target is given. The anatomy and basic operation of the ITk Strip detector is described.

The treatment of the ITk Strip detector is brief with the goal of relating only the main structures, functionalities and their integration into an exemplary next generation all-silicon tracking detector. Several scales of detector component are covered in this respect. At first the trend follows Chapter 1, inward through the global, and local supports towards the fundamental integrated readout-sensor unit, the module (Sections 2.1 and 2.2).

With the detector design in mind a number of its more interesting aspects are then examined. The general tracking ability of the Strip detector is addressed in Section 2.3. The module's non-sensor sub-components are then detailed in Section 2.4. Their function is predominantly electrical, which means that the following section (2.5) outlining the electronics architecture of the Strip detector is closely related.

In Sections 2.6 and 2.7 details of my Master's level research are discussed. First, the ITk production model is outlined to lend significance to my primary research goal of production sensor test site readiness. Details of my module-related testbeam work follow (Section 2.7). I was lucky enough to play a fundamental role in the 2016 campaign which tested the first ever irradiated full-size module (in the $130 \mathrm{~nm}$ 
process) produced Figure 2.14. This result may be the most important produced so far in proving that the ITk will be ready for the HL-LHC.

\section{$2.1 \quad$ Detector Overview}

The ITk Strip detector will boast a world record $\sim 165 \mathrm{~m}^{2}$ of silicon detecting surface, a value that is 2.6 times larger than the silicon coverage of the entire existing inner detector. It will be built to withstand the $7.5 \times 10^{34} \mathrm{~cm}^{-2} \mathrm{~s}^{-2}$ instantaneous luminosity of the HL-LHC for the ten year lifetime of the project. As seen, this translates to roughly 200 pileup events per 25ns spaced bunch crossing, an extremely hostile environment as can be inferred from Figure 1.4. The experimental duration will result in an integrated luminosity of $3000-4000 \mathrm{fb}^{-1}$ as well as an accumulated radiation fluence of $1.2 \times 10^{15} n_{e q v} \mathrm{~cm}^{-2}$ and a total ionizing dose of 50MGy in the Strip region. As seen (Section 1.5), the careful design and construction allow the new state-of-theart detector to perform as well or better than the existing detector but in the much more demanding environment of the HL-LHC.

The ITk Strip detector is an annular cylinder that fills the inner bore of ATLAS occupying the radii between $\sim 30 \mathrm{~cm}$ and $\sim 1 \mathrm{~m}$. In this respect it abuts the outer surface of the ITk Pixel system and the inner surface of the bulkheads separating the calorimeters and providing the environmental control. The layout of the full Inner Tracker system has been seen in Figure 1.8 with discussion in the accompanying Section (1.4.2).

As mentioned the ITk follows the conventional sub-system breakdown. The annular cylinder is constructed from three sub-systems; the barrel and two end-caps seen as horizontal and vertical blue lines respectively in Figure 1.8. The barrel comprises the central region of the detector extending $1.4 \mathrm{~m}$ to either side of the interaction point. It consists of four nested cylinders connected for structural support by interlinks at the ends. The endcaps on either side of the barrel increase the length of the cylinder to its full value of six meters. As seen in Figures 1.7 and 1.8 each endcap consists of six discs to allow full coverage and fulfill the tracking hit requirements. 


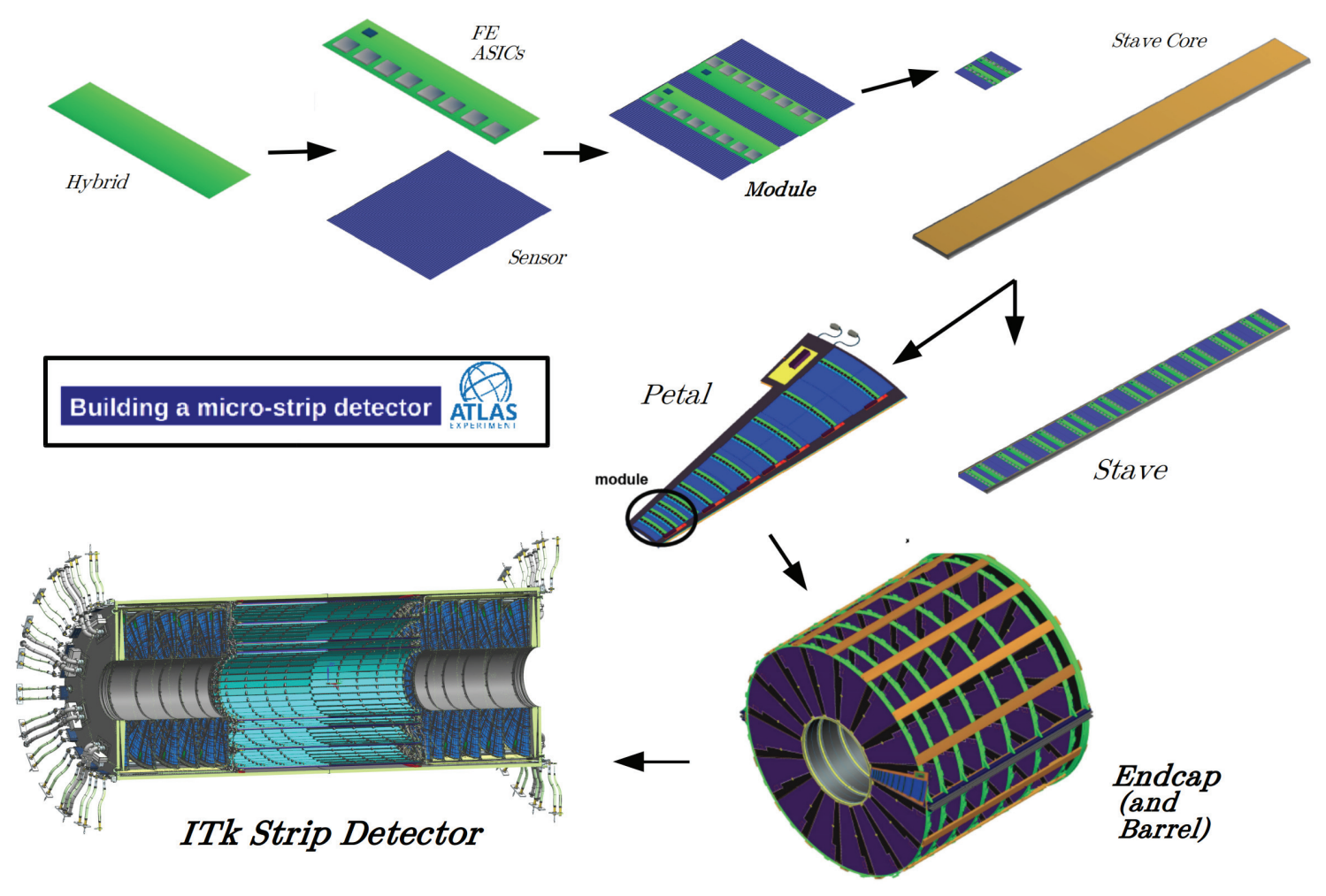

Figure 2.1: Summary of the major steps in the building of the ITk Strip detector. $[1,14]$

\subsection{Detector Architecture}

Figure 2.1 shows the ITk Strip detector hierarchy in a series titled "Building a microstrip detector" $[1,14]$. It can now be seen that the barrel and endcap are only the largest sub-systems of the ITk Strip (sub-sub-)detector. In Table 2.1 the quantity of each main component in the hierarchy is given. They are grouped into totals and their global structure placements. The locations of the barrel layers and endcap disks in the axial-radial plane ( $z r$-plane) as depicted in the layout, Figure 1.8, are given.

The barrel and endcap are constructed from the local support structures; the plank-like staves and wedge-shaped petals (respectively). These composite structures are shown in more detail in Figures 2.2 and 2.3. Thermal and electrical services are integrated directly into the staves and petals whose design incorporates geometrical and mechanical factors. Material and construction is optimized in those four respects 


\begin{tabular}{lccccccc}
$\begin{array}{l}\text { Barrel } \\
\text { Layer: }\end{array}$ & $\begin{array}{c}\text { Radius } \\
{[\mathbf{m m}]}\end{array}$ & $\begin{array}{c}\text { \# of } \\
\text { staves }\end{array}$ & $\begin{array}{c}\text { \# of } \\
\text { modules }\end{array}$ & $\begin{array}{c}\text { \# of } \\
\text { hybrids }\end{array}$ & $\begin{array}{c}\text { \# of } \\
\text { of ABCStar }\end{array}$ & $\begin{array}{c}\text { \# of } \\
\text { channels }\end{array}$ & $\begin{array}{c}\text { Area } \\
{\left[\mathbf{m}^{2}\right]}\end{array}$ \\
\hline L0 & 405 & 28 & 784 & 1568 & 15680 & $4.01 \mathrm{M}$ & 7.49 \\
L1 & 562 & 40 & 1120 & 2240 & 22400 & $5.73 \mathrm{M}$ & 10.7 \\
L2 & 762 & 56 & 1568 & 1568 & 15680 & $4.01 \mathrm{M}$ & 14.98 \\
L3 & 1000 & 72 & 2016 & 2016 & 20160 & $5.16 \mathrm{M}$ & 19.26 \\
Total half barrel & & 196 & 5488 & 7392 & 73920 & $18.92 \mathrm{M}$ & 52.43 \\
Total barrel & & 392 & 10976 & 14784 & 147840 & $37.85 \mathrm{M}$ & 104.86 \\
\hline End-cap & $\mathbf{z - p o s}$. & \# of & \# of & \# of & \# of & \# of & Area \\
Disk: & {$[\mathbf{m m}]$} & petals & modules & hybrids & of ABCStar & channels & {$\left[\mathbf{m}^{2}\right]$} \\
\hline D0 & 1512 & 32 & 576 & 832 & 6336 & $1.62 \mathrm{M}$ & 5.03 \\
D1 & 1702 & 32 & 576 & 832 & 6336 & $1.62 \mathrm{M}$ & 5.03 \\
D2 & 1952 & 32 & 576 & 832 & 6336 & $1.62 \mathrm{M}$ & 5.03 \\
D3 & 2252 & 32 & 576 & 832 & 6336 & $1.62 \mathrm{M}$ & 5.03 \\
D4 & 2602 & 32 & 576 & 832 & 6336 & $1.62 \mathrm{M}$ & 5.03 \\
D5 & 3000 & 32 & 576 & 832 & 6336 & $1.62 \mathrm{M}$ & 5.03 \\
Total one EC & & 192 & 3456 & 4992 & 43008 & $11.01 \mathrm{M}$ & 30.2 \\
Total ECs & & 384 & 6912 & 9984 & 86016 & $22.02 \mathrm{M}$ & 60.4 \\
\hline Total & & $\mathbf{7 7 6}$ & $\mathbf{1 7 8 8 8}$ & $\mathbf{2 4 7 6 8}$ & $\mathbf{2 3 3 8 5 6}$ & $59.87 \mathrm{M}$ & $\mathbf{1 6 5 . 2 5}$ \\
\hline
\end{tabular}

Table 2.1: Summary of the number of components to be used in the ITk Strip Detector, broken into barrel and endcap. [1] 
to meet the strict performance requirements of the ITk. This entails the carbon fibre body with integrated cooling loops and co-cured bus tape seen in the figures.

Both stave and petal utilize the same basic materials and only one design. Importantly, the local support structures are built as individual integrated units which are fully operable and fully testable. As can be seen in Figures 1.7 and 2.2 the local supports are quite small and this forethought logistically eases a great deal of the work during the detector construction phases. Prototyping, detector component qualification, early and late assembly stages, and commissioning, are all examples.

Each stave is inserted into the end of the barrel support structure occupying a small radial arc and half the barrel's full length. A variable number of staves is needed to make a full cylindrical layer at each of the four radii. This is seen in Tables 2.1 and 2.2. The endcaps are assembled separately from the barrel before integration and commissioning at the ATLAS surface site. Thirty-two petals make up each of the identical disk structures.

In both cases cabling, and cooling utilities are supplied to groups of nearby local supports by a total of 48 optimally packaged and routed service modules. This contributes to the ITk's remarkable material budget projections (Figure 1.9). The optimal routing is facilitated by the petals' unconventional arc-wedge shape (in lieu of a trapezoidal wedge) which couples the advantages of optimal strip orientation (radially) with optimal detector response and coverage in the geometry of the disks. As will be discussed (Sections 2.3, 4.1.2, and 4.3.2) this poses significant design constraints on the ITk Strip sensors. A matter that is at the heart of this ultimately endcap focused Master's thesis work.

Glued directly to the staves and petals are the fundamental integrated readoutsensor unit of the ITk Strip detector; the barrel and endcap strip modules. This important level of integrated unit obtains the location signature of the passing particle. Then it processes and sorts the signal within the timing limits required of the on-detector electronics (see Table 1.1). Modules are described further in Section 2.4.

The silicon coverage of the ITk Strip detector will result in the use of nearly 18000 strip modules (a staggering 13000 more than the SCT's smaller counterparts). The staves are populated with 14 modules on each side of its double faced structure (see Figure 2.3). The petals are likewise double sided utilizing nine modules per side. Eight module designs, outlined in the next section, are required for optimal 

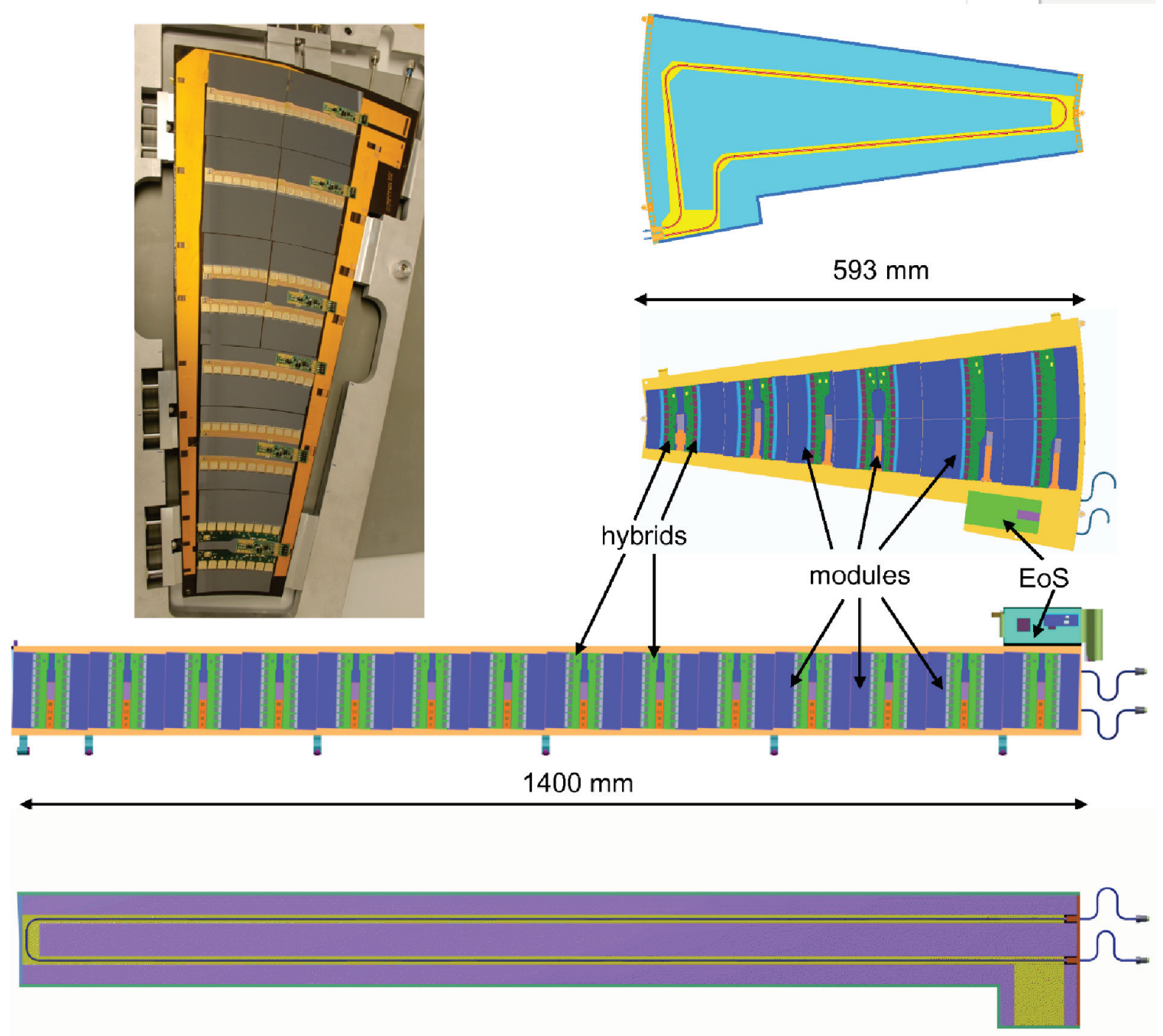

Figure 2.2: Summary of the local support structures. Bottom; the barrel stave. Top right; the end-cap petal. Top left; photograph of a thermo-mechanical petal used for finite analysis verification of thermal models. [1]

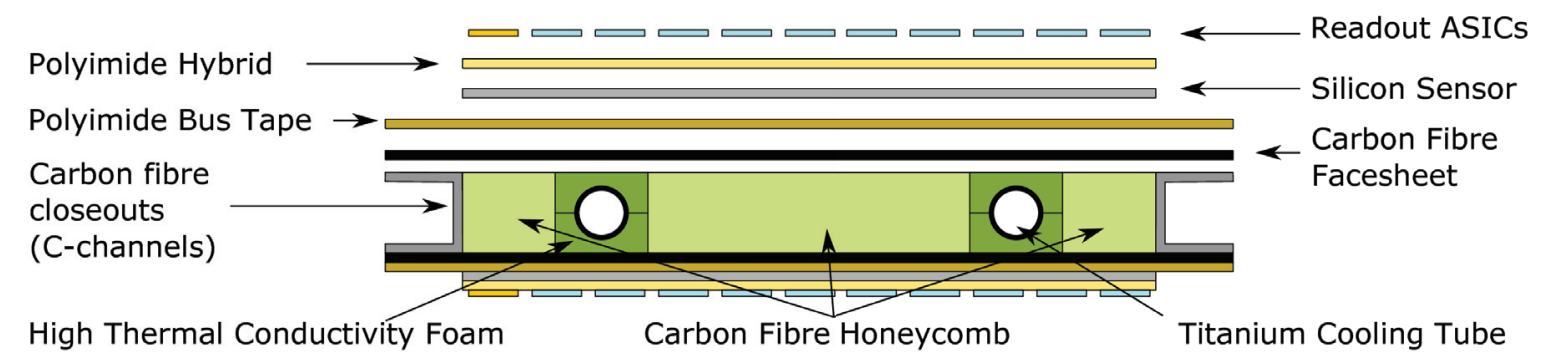

Figure 2.3: Simplified cross-sectional schematic of the stave local support system. The end-cap's petals use the same materials. Not to scale. [1] 
geometric coverage and tracking ability (they are defined by the shape of their sensor constituents, see Section 4.1.2).

\subsection{ITk Strip Tracking Functionality}

As Table 2.1 shows, the ITk Strip detector will employ nearly sixty million readout channels. These are realized as micostrips patterned into one side of the novel, large area, radiation hard silicon sensors that are the focus of the later chapters. The microstrips are of variable granularity, lengths varying from 19 to $60 \mathrm{~mm}$ with pitches ranging from 69 to $84 \mu \mathrm{m}$, values which are chosen for occupancy, readout, and geometric reasons. These details of the Strip system are tabulated; Tables 2.2, 2.3, and 4.2. They are discussed further in the context of the sensors and detector construction in Section 4.1.2.

It can be noted that the strips are quite long and that the ITk barrel staves, and endcap petals are double sided (Section 2.2). This necessitates development of the concept of single "point" measurements in one silicon layer mentioned as the fundamental "hit" unit used in track reconstruction (Section 1.5.1). In the ITk Strip detector the use of small offset angles in the normal orientation of the microstrips has allowed the track reconstruction and occupancy capabilities discussed. In this respect the two sides of each barrel layer or endcap disk yield a single 2-D point measurement with an efficient and vast improvement of the resolution in the secondary coordinate (which would have otherwise been near to the lengthy strips). The small angle is termed "stereo" and yields a sharp-diamond grid when overlaid with the opposing side of the local support. A value of $26 \mathrm{mrad}$ is used on the barrel staves and $20 \mathrm{mrad}$ in the endcap sensors.

The solenoid magnet that dominates the magnetic field in the central ATLAS volume that the ITk occupies is aligned along the beam axis. Charged particles are bent in the transverse plane as they explode outward from the interaction region. Microstrips in the central barrel region of the ITk are aligned parallel to the beam axis to provide the primary cylindrical $\phi$ coordinate measurement. The 26mrad stereo angle, obtained by placing rotated modules on the stave supports (see Figure 2.2), allows the secondary axial $z$ to be determined reasonably well. The remaining radial coordinate is given by the barrel layer position. Hermiticity and space considerations result in the tilting of the staves in the transverse plane (width-wise) to allow 


\begin{tabular}{llllll} 
Layer & $\begin{array}{l}\text { Radius } \\
{[\mathbf{m m}]}\end{array}$ & $\begin{array}{l}\text { Channels } \\
\text { in } \boldsymbol{\phi}\end{array}$ & $\begin{array}{l}\text { Strip } \\
\text { Pitch }[\boldsymbol{\mu m}]\end{array}$ & $\begin{array}{l}\text { Strip Length } \\
{[\mathbf{m m}]}\end{array}$ & $\begin{array}{l}\text { Tilt Angle } \\
{\left[{ }^{\circ}\right]}\end{array}$ \\
\hline 0 & 405 & $28 \times 1280$ & 75.5 & 24.1 & 11.5 \\
1 & 562 & $40 \times 1280$ & 75.5 & 24.1 & 11.0 \\
2 & 762 & $56 \times 1280$ & 75.5 & 48.2 & 10.0 \\
3 & 1000 & $72 \times 1280$ & 75.5 & 48.2 & 10.0 \\
\hline
\end{tabular}

Table 2.2: Details of the barrel sensor geometry in terms of the four layer structure. Radius is with respect to the beam axis and tilt angle is with respect to the $r \phi$ plane. Each barrel layer is $2.8 \mathrm{~m}$ long centered on $z=0$. [1]

overlapping.

Table 2.2 summarizes the details of the central barrel region as shown in the ITk layout; Figure 1.8. A constant strip pitch of $75.5 \mu \mathrm{m}$ is implemented across the barrel. While due to the aforementioned granularity considerations two strip lengths are used; $\sim 2.5 \mathrm{~cm}$ and $\sim 5 \mathrm{~cm}$, for the inner two layers and outer two layers, respectively. These values and their endcap counterparts, seen in Table 2.3, result in the occupancy patterns observable in the bottom plots of Figure 1.13. For the barrel the two strip lengths are each associated with one of the eight sensor and module designs that make up the fundamental detector units.

In the annular disk environment of the endcaps the geometry constraints are much more onerous. The primary coordinate measurement required is again the $\phi$ angle in the bending plane oriented transverse to the axis of the colliding beams. ${ }^{1}$ The optimal microstrip orientation is radially outward from the beam pipe now with a constant angular pitch. The superposition of the two stereo angles implemented on either side of the petals provides the radius measurement in conjunction with the beam-axial value given by the disk position.

Table 2.3 summarizes the endcap related strip granularity details. The disk structure is replaced by the six radial rings that are implemented in the modules and further classified by the strip rows implemented in the corresponding sensors (see Section 4.1.2). Geometry and sensor manufacturing forces the strip lengths and pitches but the general trend of longer strips at larger radii remains. The sensors that comprise the ringed disk petals and define the Strip module designs are seen in Table 4.2. With

\footnotetext{
${ }^{1}$ The positive $\mathrm{x}$-axis in the ATLAS coordinate system points towards the center of the LHC ring. [8]
} 


\begin{tabular}{llll} 
Ring/Row & $\begin{array}{l}\text { Inner Radius } \\
{[\mathbf{m m}]}\end{array}$ & $\begin{array}{l}\text { Strip Length } \\
{[\mathbf{m m}]}\end{array}$ & $\begin{array}{l}\text { Strip Pitch } \\
{[\boldsymbol{\mu m}]}\end{array}$ \\
\hline Ring 0 Row 0 & 384.5 & 19 & 75.0 \\
Ring 0 Row 1 & 403.5 & 24 & 79.2 \\
Ring 0 Row 2 & 427.5 & 29 & 74.9 \\
Ring 0 Row 3 & 456.4 & 32 & 80.2 \\
\hline Ring 1 Row 0 & 489.8 & 18.1 & 69.9 \\
Ring 1 Row 1 & 507.9 & 27.1 & 72.9 \\
Ring 1 Row 2 & 535 & 24.1 & 75.6 \\
Ring 1 Row 3 & 559.1 & 15.1 & 78.6 \\
\hline Ring 2 Row 0 & 575.6 & 30.8 & 75.7 \\
Ring 2 Row 1 & 606.4 & 30.8 & 79.8 \\
\hline Ring 3 Row 0 & 638.6 & 32.2 & 71.1 \\
Ring 3 Row 1 & 670.8 & 26.2 & 74.3 \\
Ring 3 Row 2 & 697.1 & 26.2 & 77.5 \\
Ring 3 Row 3 & 723.3 & 32.2 & 80.7 \\
\hline Ring 4 Row 0 & 756.9 & 54.6 & 75.0 \\
Ring 4 Row 1 & 811.5 & 54.6 & 80.3 \\
\hline Ring 5 Row 0 & 867.5 & 40.2 & 76.2 \\
Ring 5 Row 1 & 907.6 & 60.2 & 80.5 \\
\hline
\end{tabular}

Table 2.3: Details of the endcap sensor geometries. Parameters are given for each strip segment of the petal. Inner radius are in respect to the beam axis and the listed strip pitch is the length averaged value. [1] 
the first three rings occupied by one sensor and the remaining three by two, one arrives at the numbers given therein and in the count of components above (Table 2.1). The readout paraphernalia associated with the module scale (seen in Table 2.1 and discussed in Section 2.4 below) are dispersed about the petals in a manner congruent with the microstrip arrays and readout latency obligations.

The arc-wedge or fan shaped petals used in the ITk endcaps are an innovation that comes at the cost of increased difficulty in the implementation of the required strip configuration in what is already the more difficult part of the detector to build. The ITk Strip detector will use a novel sensor shape, the "stereo annulus" geometry, described in detail in Section 4.3.3. This allows the integration of the $20 \mathrm{mrad}$ stereo angle directly into the silicon sensors with radial, equal length strips. The advantages in the coverage and tracking are multi-fold. This sensor type is intimately related to this thesis work as the current iteration of ITk Strip sensor prototype (see Chapter 4 ) is the first stereo annulus silicon microstrip sensor ever produced. The potential of this novel sensor shape is considerable and some of the very first results for these devices were obtained in the canon of this Master's work, these are shown in Sections 5.3 and 5.5 .

It should be noted that in the ITk Strip detector clustering algorithms will be used in conjunction with a binary readout; the ABCStar front-end ASIC discussed in the next section. Binary readout entails that the only information received from a channel will be a charge over threshold signal (ie. a binary, hit or no hit indication). This differs from the more electronically taxing analog measurement where actual charge information is obtained in each channel. This more informative technique which allows smaller area cluster centroid measurements is planned for the higher resolution needs of the inner radii's ITk Pixel detector.

\subsection{The ITk Strip Module}

The fundamental integrated readout-sensor component of the ITk Strip detector is the Strip module, photos of the most recent ITk Strip prototypes in their custom green test frames are seen in Figure 2.4. The module, as the building diagram showed (Figure 2.1) is composed of the basic components of sensor, "hybrid", and "FE (frontend) ASIC". The quantities of these are tabulated in Table 2.1. As seen, module and sensor can be taken equivalently, as will be seen, so to can FE ASIC and ABCStar. 


\begin{tabular}{lllll} 
Acronym & Full Name & Basic functionality & Prototype & $\begin{array}{l}\text { Production } \\
\text { Chip }\end{array}$ \\
\hline ABC & ATLAS Binary Chip & $\begin{array}{l}\text { Converts incoming charge signal into } \\
\text { hit information }\end{array}$ & ABC130 & ABCStar \\
HCC & $\begin{array}{l}\text { Hybrid Controller Chip } \\
\text { Autonomous Monitor } \\
\text { and Control Chip }\end{array}$ & $\begin{array}{l}\text { Provides monitoring and interrupt } \\
\text { functionality } \\
\text { AMAC }\end{array}$ & HCC130 & HCCStar \\
FEAST & FEAST & AMAC-I & AMAC-II \\
\hline
\end{tabular}

Table 2.4: Summary of the custom ASIC set used on the ITk Strip module. [1]

This view of the module is expanded in the exploded inner barrel module depiction shown in Figure 2.5. With 17888 modules required for the ITk Strip detector (not counting spares) their design must be extremely reliable in all the varied respects that have been seen. It must also be optimized for mass production and low cost. The eight sensor-shape-based, module geometries have been discussed in a tracking respect (Section 2.3) and are discussed further in Section 4.1.2 in a sensor and detector respect. Since different module configurations utilize the same basic components with only geometric implementation changing they will not be discussed any further here. The basic material budget for the module was shown in Figure 1.9 using prototype measurements.

The modules' non-sensor related functionality centers around its electrical abilities. These are briefly outlined in the following, closely related, section on the overall electronics architecture of the Strip detector (Section 2.5). Here the focus will be on relating the basic practicality and inter-relation of the components seen in the exploded view.

The most essential electronics functions are shown for context in Table 2.4. In this document the module-scale focus is the integral signal processing function of the ABCStar which is examined more closely in relation to recent prototype results in Section 2.7. Some of these (Section 2.7.3) were obtained in the context of my 2016 testbeam involvement, a large secondary aspect to my Master's level research on the sensors.

Figure 2.5 shows that the sensors are the main substrate upon which the modules are built, a thermal consideration taking advantage of the local support composition seen in Figure 2.3. Mounted directly to the silicon using electronics grade insulative 

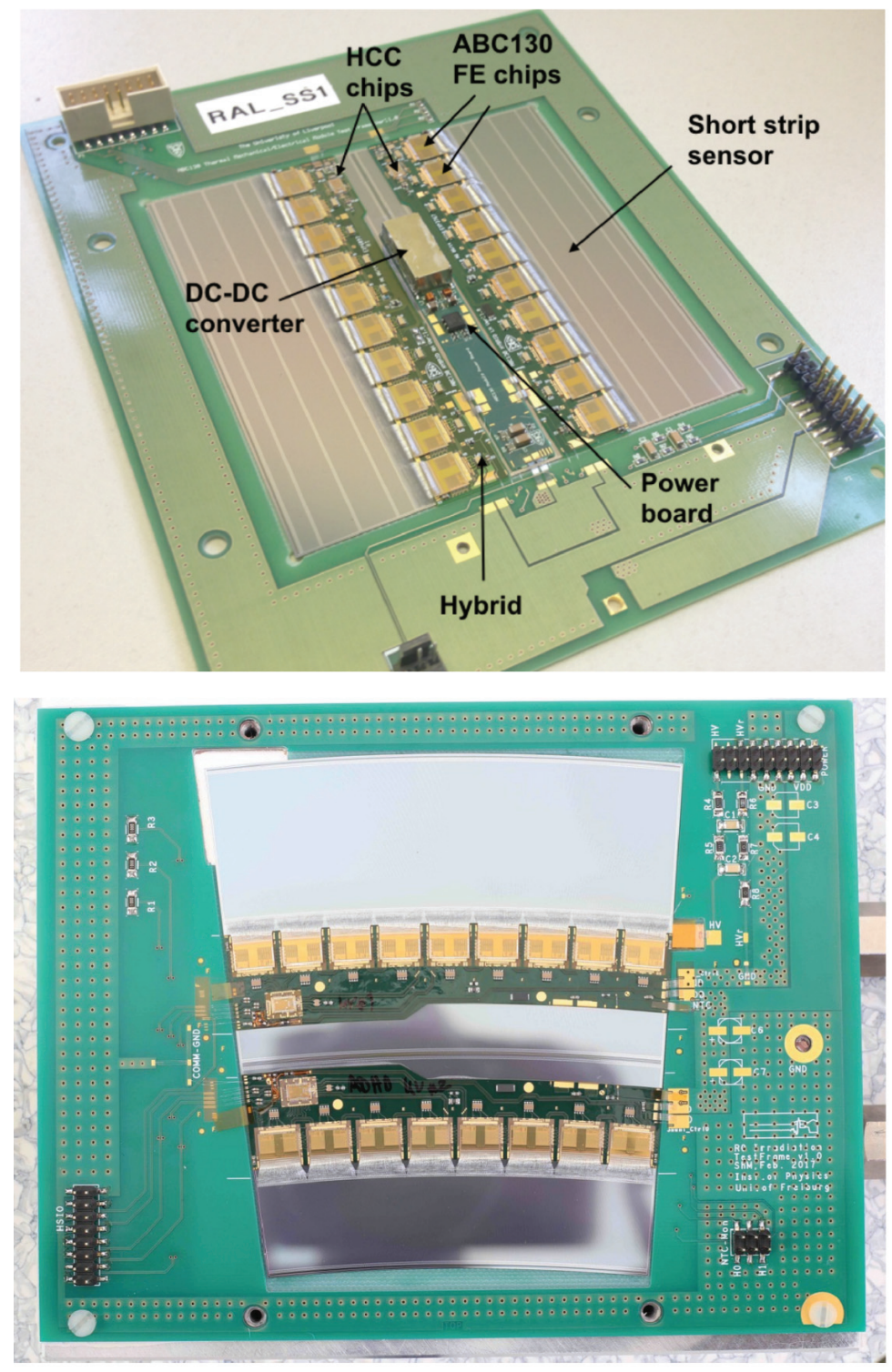

Figure 2.4: Photographs of recent ATLAS ITk prototype modules. Top; a short strip barrel module with ATLAS12 sensor. Bottom; an innermost ring (R0) endcap prototype module with ATLAS12EC sensor. See Chapter 4 for discussion of sensor types. [1] 


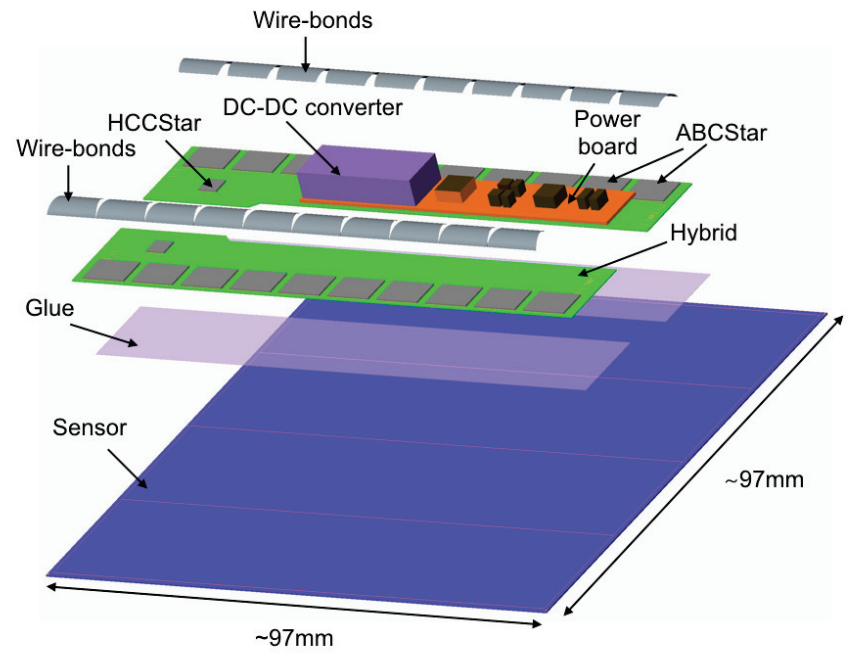

Figure 2.5: A short strip barrel module in exploded view. All major components are shown and are found in both barrel and end-cap modules. [1]

epoxy are up to three printed circuit boards (PCB) in two types. Each module will have a power board and either one or two "hybrids" in numbers congruent with the microstrips on each sensor. The custom, radiation hard, kapton-flex "hybrid" PCBs host the most important data related electronics at the module level; the ATLAS Binary Chip (ABCStar) front-end ASIC and its stave interfacing brethren, the Hybrid Control Chip (HCCStar). These custom application specific integrated circuits (ASICs) are developed in the $130 \mathrm{~nm} \mathrm{CMOS}^{2}$ process, a circuit density and radiation hard innovation in regards to the SCT's 250 $\mathrm{nm}$ nodes. Triplicated logic to protect against data corruption due to radiation induced bit flips ("Single Event Upsets" or SEUs) in the ASICs is also used.

Individual wire bonds connect the roughly 60 million strips to the $\sim 235$ thousand ABCStar which each have 256 front end (FE) channels. The signal from 128 strips of two neighbouring ring segments are collected by one ABCStar through a four row FE pad arrangement allowed by the smaller ASIC processing. The evaluated binary signal is then collected from the ABCStar by the HCCStar which further packages the data to be sent off-module (again by wire bond) to the local structures' integrated bus tapes. Up to 12 ABCStar sit on each hybrid (with one HCCStar controller).

\footnotetext{
${ }^{2}$ Complimentary Metal-Oxide Semiconductors (CMOS) implemented in logic circuits use abutting $\mathrm{n}$ and p-type MOS Field Effect Transistor (MOSFET) structures as developed over Chapter 3 and discussed in Section 3.3.6 more specifically.
} 
The "Star" post-script signifies the move to a parallel communication scheme between the ABCStar and the HCCStar. This replaced the serial communication, implemented in the SCT and the current iteration of prototype chips (the ABC130), that saw half a hybrid's worth of front-end chips 'daisy-chained' on one data line. Latency, occupancy, and bandwidth projections have shown that the move to the Star readout scheme is an integral change to the front-end (only a max of three linked 130nm ABC chips would be readable otherwise). It also allows geometric considerations to be more easily implemented like the use of wire-bond linked hybrids in the abutted modules in the outer three endcap rings. There the two HCCStar sit on one side for bus tape routing.

The powerboard is the other sensor mounted PCB, it not only hosts the module powering electronics but an advanced systems monitoring and control system. The powering scheme at the module level is complicated by the required use of existing ID cables in the ITk. The huge increase in channel numbers requires a much greater low voltage (LV) consumption (for chip powering and data propagation since a differential signal must be used to negate interference effects). The LV concerns are overcome by the use of step-down DC-DC buck converters on the module powerboard which allow higher voltages to be used in the servicing links. Again, radiation hardness for the HL-LHC's unparalleled integrated luminosity necessitates the use of novel technology. A custom ASIC has been designed to meet this and requirements in size, inductive interference, and operability. This new device has been designated upFEAST and joins the Autonomous Monitor and Control (AMAC) chip to round out the main module level custom ASIC set, Table 2.4.

The AMAC provides integrated safety and functionality interlocking ability with both environmental and electrical monitoring. A vital operation is its power-up sequence utility which controls not only the LV to power the ASICs but the high voltage (HV) supplied to the sensors as well. This allows the effective use of the existing, numerically deficient HV cables; requiring up to four sensors on one line. The key innovation in this case is a $\mathrm{HV}$ multiplexing circuit on the powerboard and a dedicated communication protocol to allow AMACs to turn off (and/or on again) problematic, neighbouring modules. This not only curtails the potential loss of all four modules on the HV line if one sensor develops high current issues (via breakdown, see Section 3.3.5) it gives added operational maneuverability, these allow the ITk to meet its goals in long-term, remotely monitored reliability. 


\subsection{Overall Electrical Architecture}

The treatment of the electronics structure presented in this section is exceedingly brief in relation to its complexity in the actual ITk. The intension is only to add a little colour to the mostly functional and mechanical based discussions above, before the dive into the fundamental tracking silicon that is the namesake of this Master's thesis. The focus in this respect is to describe the main pathways for data and power in the Strip detector. The logical progression is opposite to the thesis' general inward trend; outward from the module level footing of the previous section. The discussion is rooted in the technical schematics seen in Figure 2.6 which offer the interested, magnifier-laden reader a much more detailed glimpse of the module and stave level electronics architecture than is attempted in the text.

The voltage signal induced on the metal strips by the passage of an ionizing particle through the silicon bulk is registered in some of the 256 channels of the ABCStar where it is amplified, shaped, and discriminated against an optimized threshold to get a binary, 1 or 0 , hit. Zero suppression is used in the ABCStar in conjunction with the Level-0 and Level-1 trigger buffers and cluster finding logic circuitry. Data is sent to the HCCStar in parallel from all ABCStar and the timing, trigger, and control (TTC) signals' opposing path is similarly routed. A $40 \mathrm{MHz}$ (25ns) clock is used.

The HCCStar builds the data-packets to send (unprocessed) through the AMAC and, eventually, off-detector. As mentioned the data signal is sent in differential form. A challenging $640 \mathrm{Mbit} / \mathrm{s}$ data downlink on the local structure bus tapes results from the required move to the parallel Star data streams.

As Figure 2.6 shows, the powerboard interfaces all objects on the module to each other and to the local supports. The AMACs eponymous nature leading itself to nearly exhaustive module-level connection and influence. The main connections shown at the module level (Figure 2.6 top) are the yellow monitoring and control and brown calibration circuitry. These are joined by the blue, purple, and pink connections of the larger detector control systems (DCS). The aforementioned powering scheme (Section 2.4) is shown by the green and red respectively signifying low and high voltage routing.

The electronics architecture at the local support level is shown in the bottom half of Figure 2.6 for one symmetric side of an inner layer barrel stave. The 14 modules are contracted to three; the generic one (shown in the upper magnified view) and 


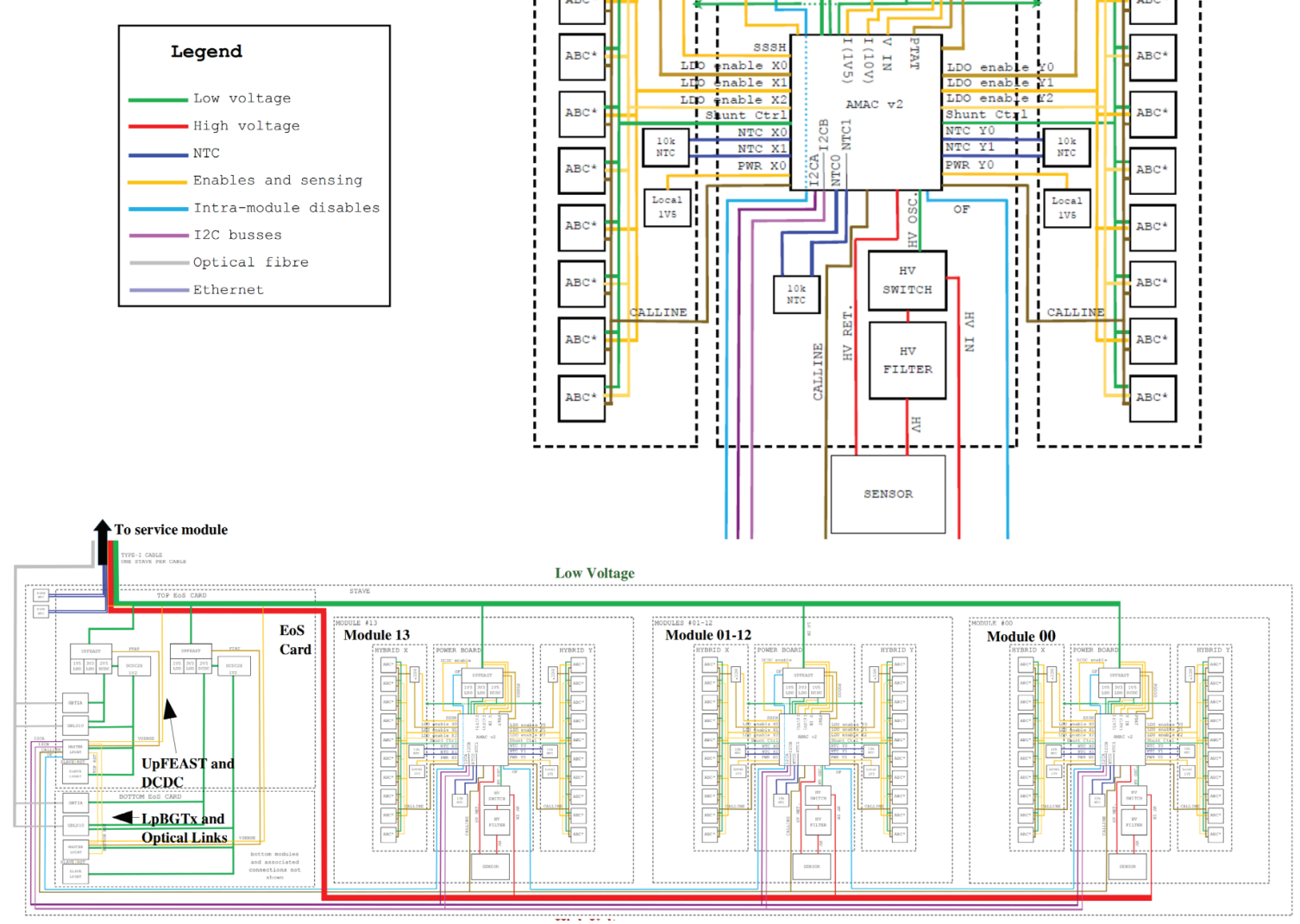

Figure 2.6: Technical schematics of the electrical architecture. Top; at the (short strip barrel) module level. Bottom; at the (inner) stave level. Aspects of the schematic are discussed in the accompanying text (Section 2.5). [15] 
the two (for signaling purposes) slightly different end modules. The TTC and data signals follow the green low voltage path while the HV required to put the solid state detectors into a tracking capacity is routed with the DCS controls. As discussed further in the following chapters, potentials of 500 to $700 \mathrm{~V}$ are necessary for sensor operation and constitute one of the major detector design challenges (especially with the rise in $\mathrm{HV}$ currents that occurs with silicon radiation damage).

Seen at the very left of the stave's technical schematic and in the local support summary figure (2.2) is the End of Structure (EoS) card. The EoS card's main function is the conversion of the low voltage differential data signal (LVDS) coming from the HCCStar to a number of ten gigabit per second optical links that are shipped out of ATLAS. The main features are labeled generally in the schematic. More rad hard DC-DC converters power the informatively named low power GigaBit Transceiver (lpGBTx) which interfaces the LVDS signal to the Versatile link (VTRx+) fibre optics driver.

Not shown in the electrical schematics are the service modules mentioned in the system architecture which not only connect the EoS cards to the patch panels outside the ITk but provide the formal ground reference location in the system.

\subsection{ITk Strip Detector Production Model}

The staggering number of requisite parts in the larger of the two inner sub-detectors, Table 2.1, and relatively short time-scale of the project, Figure 2.7, necessitates optimization of the production procedure with a focus on large-scale ease of construction and affordability. This has supported the use of a system based on the fully functional, small size local supports. Crucially offering the ability of parallel, "systemslike" (realistic) testing without the need for larger infrastructure simplifies essentially all aspects of the detector production and assembly. Detector-wide use of two local supports and a few fundamental module designs hosting identical basic components has allowed mass production and automated assembly approaches to be likewise implemented. The production model of the ITk Strip detector is deeply rooted in these system concepts and the reliance upon parallelization and industrialization they entail.

For accounting and feasibility purposes the work for the ITk project is broken into 
ten practically sized "work packages". These cover varying overarching responsibilities; sensors, read-out chips, modules, local support electronics, fully assembled local support, global mechanics, services, integration, off-detector electronics, and component evaluation. These classifications serve as the basis for the organization of the collaboration. In Figure 2.7 a Gantt chart representation of the main milestones of the ITk Strip project is shown with tasks divided into the work package classification scheme.

While nearly 10 years from technical design report to first beam, the majority of the actual production and assembly of the Inner Tracker will take part in a three and half year span. During this short assembly period, collaborators will need all the time offered by the automated and parallel production scheme. Examples of this already implemented include multi-hybrid testing panels, pick-and-place hybrid population, robotic module loading, and plans for multiple test sites per production site.

In Figure 2.8 a simplified scheme for the product flow is shown. The production model accounts for yield estimates, transportation periods, site to site variability in production ability, and incorporates estimates of the production rates and required man hours.

Over 20 institutes from across the world, including several in Canada, will take part in the ITk assembly. For logistics purposes, the institutes are grouped into two clusters, one responsible for the barrel production and one for the endcap. Each cluster works primarily amongst itself in-line with the scheme laid out in Figure 2.8. Different aspects of the assembly and quality testing are completed in parallel at different institutes and shipping of components between institutes occurs within the cluster. However, again for logistics, it is foreseen that the responsibilities for some tasks may be undertaken by one cluster or the other. Some such items are the dicing and testing of a given ASIC, the HV multiplexer and EoS card fabrications, and a sub-set of the sensor testing.

\subsubsection{Canada's Role in ITk Production}

The Canadian collaboration will serve as a member of the endcap assembly group. Roughly one quarter of the endcap modules will be assembled and mounted into their petal supports in Canada ( 1700 modules). The components, separate and 


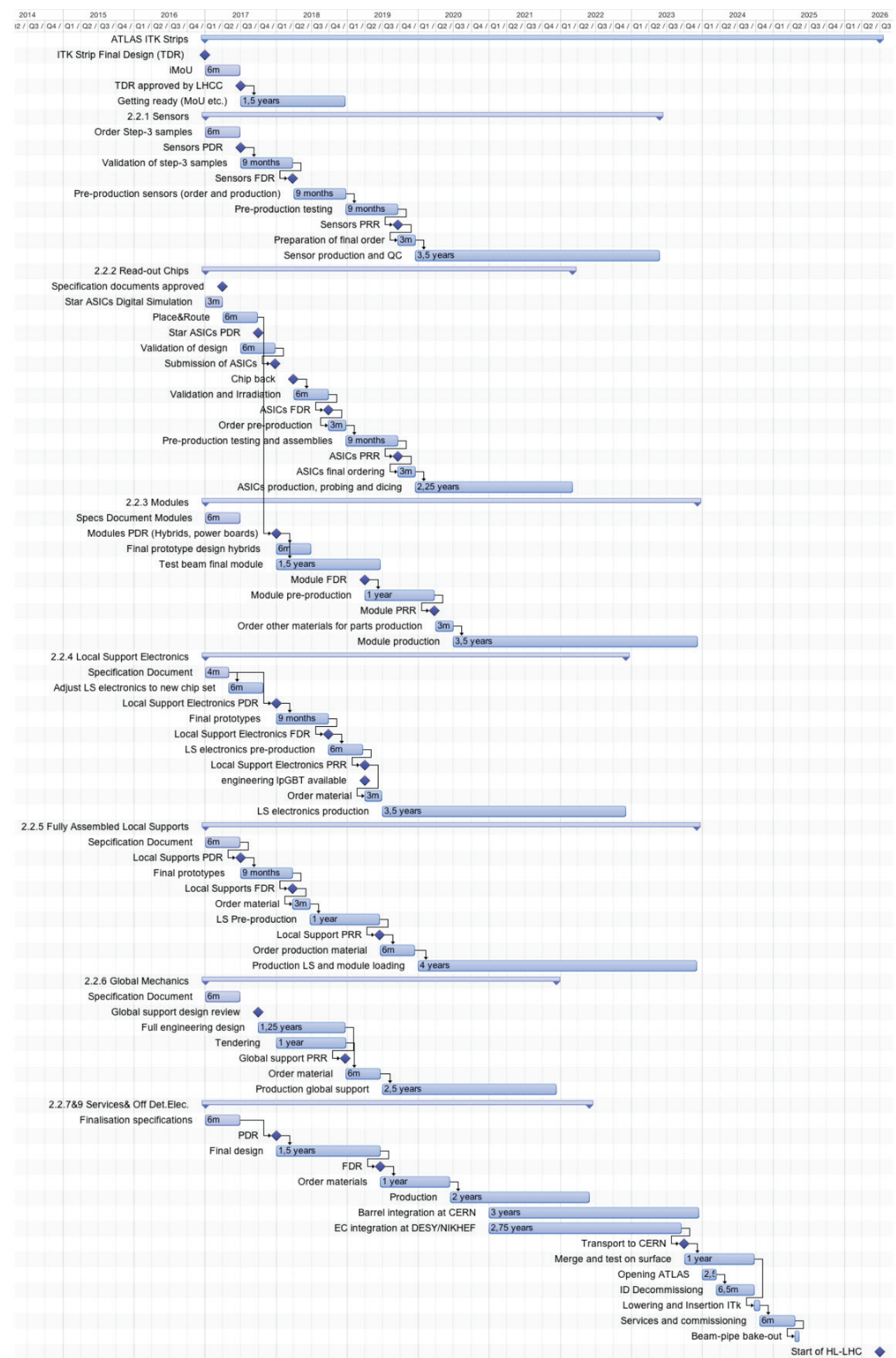

Figure 2.7: Gantt chart representation of the ITk Strip Detector major milestones. Classification in terms of work packages is given. The long-lead time sensors compose the vanguard of the development. [1] 


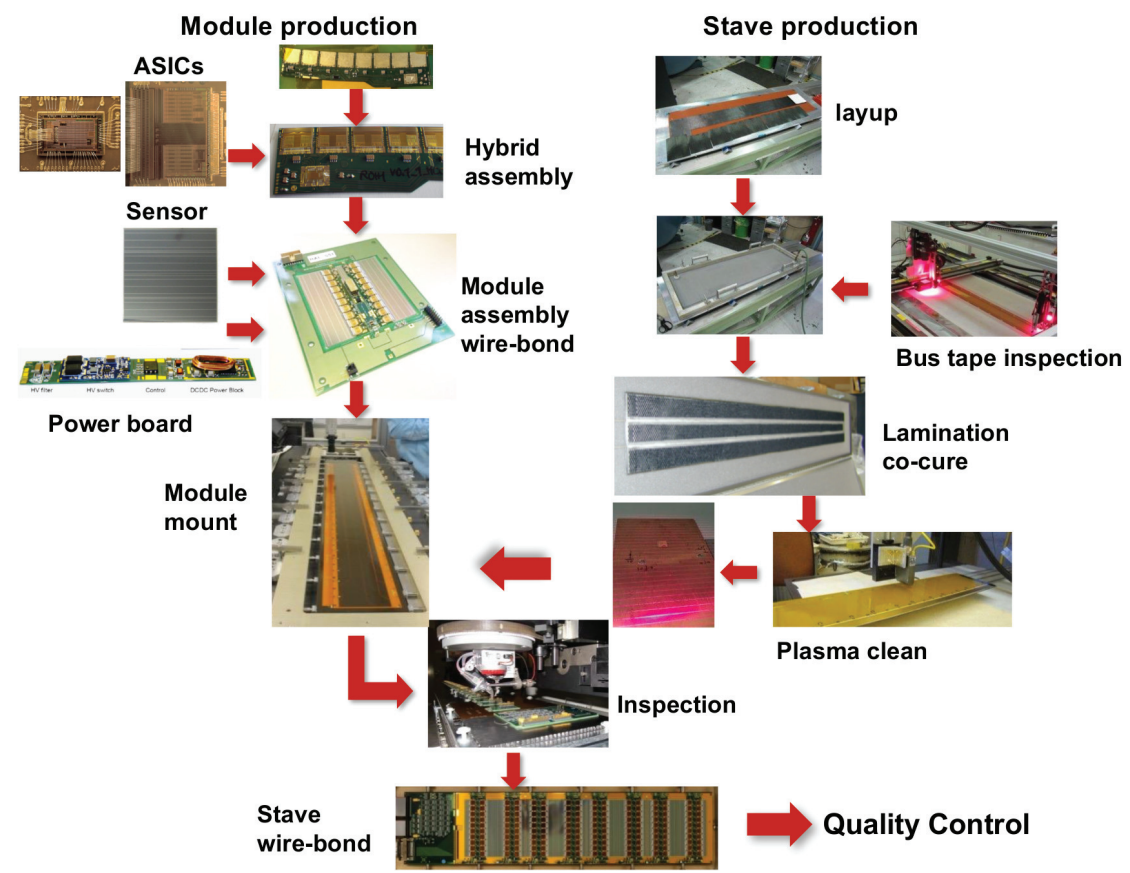

Figure 2.8: A simplified schematic of the production scheme of the ITk Strip Detector local support structures. Each step is conducted in parallel and detailed aspects of the testing program are not shown. [1]

assembled, will be subject to strict quality testing at most stages in the fabrication process.

Two clusters, geographically situated, form naturally from the Canadian institutes. In each cluster there is one main assembly site. The remaining sites will focus on the variety of time consuming testing procedures that must be performed at each stage of assembly. Module assembly (hybrid population, sensor-hybrid gluing, wirebonding) for the eastern cluster will happen at the University of Toronto working with a Toronto based industry partner (Celestica). In the west, module construction is done in-house at the TRIUMF laboratories. Mounting of the assembled endcap modules into the petal structures will likewise be conducted at TRIUMF.

At Carleton University the ITk Strip production effort will be three-fold. Firstly, it will see ITk Strip production modules as the designated trouble shooting location in the Eastern cluster. Secondly, dicing and probing of the Canadian ABCStar ASICs is expected to occur at an Ottawa based company with a Carleton University liaison. And, of course, third, the testing of the novel, large area, radiation hard microstrip sensors will be conducted at Carleton University. 
The largest contribution by far is as a pre-assembly sensor testing site for all the Canadian endcap sensors. Furthermore, with the sensor vendor wishing to optimize the production communication channels, Carleton University will become one of only four such sites worldwide. As a result it will see a throughput of sensors far exceeding that required for Canadian modules; roughly 5000 sensors will pass through its doors in all eight geometries. The preparation of the Carleton University site is the real topic of my Master's work. The infrastructure, expertise, and technique developed by me serving as the basis for that which will be used on the thousands of ATLAS ITk Strip production sensors. Since experience at the site was limited at the start of development the knowledge collected in this document is a crucial part of my sensor-related work.

\subsection{Prototype Results}

To wrap up the more general discussions of the HL-LHC, ATLAS, ITk, and ITk Strip scales offered in this and the previous chapter a brief look at non-sensor results of recent ITk Strip prototypes will show that the promises proffered by the ITk and ITk Strip design will be met. The discussion will revolve around the grueling end-oflifetime performance constraints.

The current prototype stage is an integral one associated with the recent release of the Strip detector's Technical Design Report (TDR) [1]. Full electrical barrel stave prototypes are the cutting edge with thermo-mechanical test proxies of both local supports thoroughly tested already (valuable input for thermal simulation projections). Barrel and endcap modules have also been constructed and taken to testbeam with the expected results. Modules irradiated to various fluence with various particle types have likewise been tested. These test results have been used as input for the next iteration of prototypes which for many components will be the last before production.

The 2016 beam tests saw many firsts, foremost of which was the first full-size irradiated $130 \mathrm{~nm}$ based module. Involvement in the 2016 testbeams was the second largest contribution to my Master's level research. I was fully involved in both testbeams of the year. Under the ATLAS authorship qualification task directive of "testing and documentation of irradiated devices" I became intimately involved with the first tests of the irradiated module and the important results presented here 
(Figures 2.13 and 2.14).

The fundamental, $3000 \mathrm{fb}^{-1}$ realm of "end-of-lifetime" testbeam results are augmented by recent performance-related radiation hardness advances obtained with the ABCStar chip (Figure 2.11). These are discussed prior to the testbeams following a more basic discussion of the processing inherent in the front-end chips of a microstrip detector. This necessary detector theory is related to the "S-curve" (Figure 2.9) and "response curve".

The key,2016 testbeam, end-of-lifetime results alluded to are the signal to noise ratio for irradiated and unirradiated full-size module prototypes, Figure 2.14. The early, disadvantaged ITk Strip prototype is seen to pass the $10: 1$ requirements necessary for optimal track reconstruction. Detailed studies allow the estimation of the true end-of-lifetime performance for the Strip detector (Table 2.5).

It is useful to note again for the following that the current iteration of ABCStar prototype is the $\mathrm{ABC} 130$.

\subsubsection{ABCStar Signal Processing}

The Z-shaped S-curve is shown in the left side of Figure 2.9. It is the result of a scan in the discriminating threshold voltage of the $\mathrm{ABC}$ front-end chips (the x-axis), which calibration allows to be expressed in variously weighted electronics count, millivolt, and femto-Coloumb equivalents. The efficiency being quantified by the y-axis is the ABCStar's ability to collect an expected hit at a given threshold (ie. its average hit rate). The full efficiency is known theoretically since the S-curve is a characterization tool used in conjunction with self-injected charge for chip calibration or with known particle irradiation in a module setting for performance analysis. These two cases constitute the examples discussed in the following subsections.

The S-curve's characteristic shape is the result of what it is measuring; noise and signal. These are discussed in more detail in a sensor context in Chapter 3 (specifically Sections 3.3.7 and 3.2 or 3.3, respectively). The random noise arising in and on the sensor collected at the front-end results in a Gaussian shaped voltage pulse while the signal induced by the passing particle is described by the well understood Landau function. The efficiency curve is then naturally interpreted as the integral of the convoluted signal and noise distributions. In a correctly calibrated, unirradiated chip the result is seen in Figure 2.9; high efficiency at the low thresholds caused by signal 


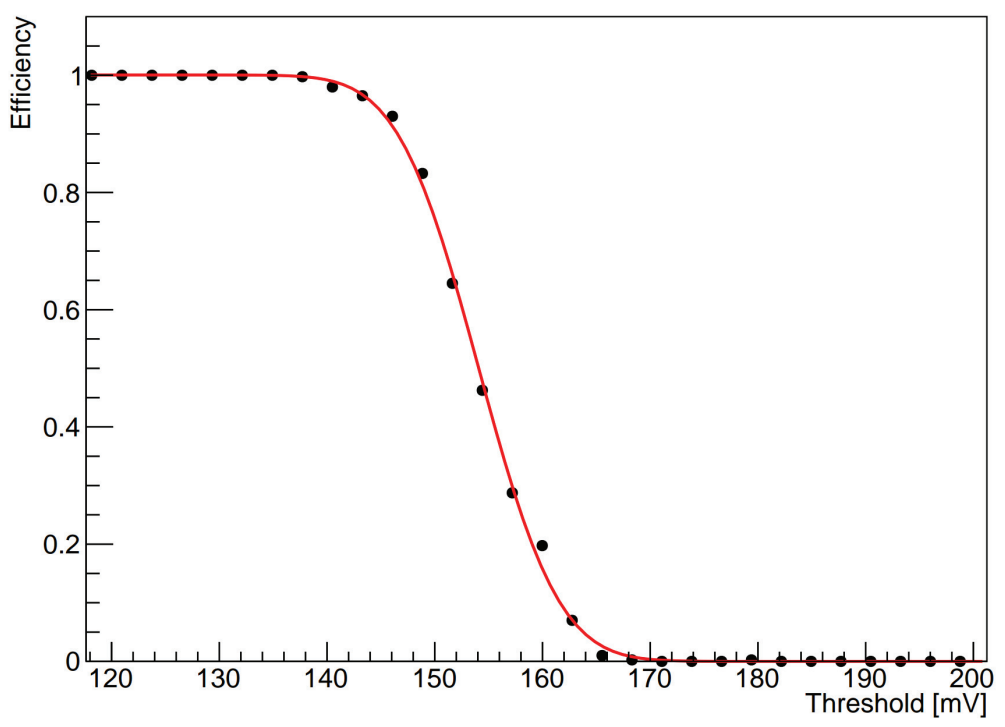

Figure 2.9: Exemplary hit efficiency vs. binary signal threshold "S-Curve". Data is an $\mathrm{ABC} 130$ with an injected calibration charge of $1.51 \mathrm{fC}$. [1]

and noise giving way to a smooth efficiency drop associated with the signal's Landau peak. The $50 \%$ efficiency threshold value, termed "Vt50", is equivalent to the median injected charge and defines the threshold around which calibration and performance analysis are centered.

Fitting the S-curve (with a skewed error function) allows both the output noise and signal or inputted charge to be determined. The front-end chip is further characterized by repeating the process for several input calibration charges which allows the input noise and gain to be extracted. The plot in this case is called the response curve. It is the Vt50 value as a function of input charge. The gain is obtained from the slope of the plot and is divided by the averaged noise value (from the S-curves) to obtain the input noise charge. This quantity defines the Equivalent Noise Charge (ENC, given in electrons) that is the (calibrated) charge required at the FE to produce the noise level observed at output.

\subsubsection{The TID Bump and the ABCStar}

This subsection details a recent radiation hardness advance made moving from the ABC130 to ABCStar design. This is related to an effect in which the digital current of the module has been observed to increase (and then decrease back to baseline) with total ionizing dose (TID). The characteristic, temperature and dose rate dependent 


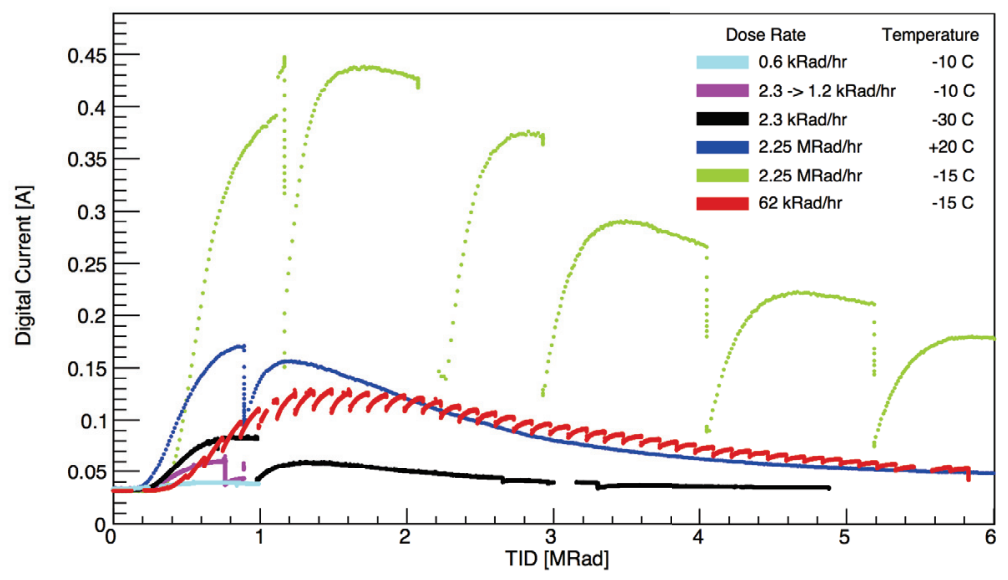

Figure 2.10: "The TID Bump". Digital current of the module versus total ionizing dose for ABC130 front-end chip prototypes. Dose is delivered by X-rays at the rates and temperatures prescribed. [1]

shape is called "the TID bump", it is seen in Figure 2.10. The underlying physics at fault is the well understood (Section 3.3.6) formation of parasitic pathways in the transistors that make up the logic circuitry in the front end chip. ${ }^{3}$ This is an issue that is fixed with the ABCStar implementation of enclosed transistors. This and other innovations are being tested currently with the first ABCStar preliminary, low-count prototype run.

The move to enclosed transistors is required for optimal performance at end-oflifetime in the ITk Strip detector's radioactive environment. This is summarized in Figure 2.11. Gain and noise measurements of ABC130 and ABCStar versus the total ionizing dose are shown. The left plot shows that the gain follows a trend that mirrors the TID bump (down then back to baseline). While in the centre plot the input noise in the ABC130 is seen to rise, problematically, without return. This effect is mitigated significantly in the ABCStar, as seen in the right plot of Figure 2.11. This ensures that the signal to noise requirements are met with good margin even after ten years of high luminosity operation.

While the effects of TID are reduced in the ABCStar its influence on the overall

\footnotetext{
${ }^{3}$ The transistors are n-type MOS structures. The TID bump related parasitic pathways are through the insulator at the edge of the device where ionizing radiation damage will have caused interface state buildup. The return to baseline is caused by the filling of the interface states at rates exceeding their creation. The analogue circuitry is not affected due to larger transistor size. [1]
} 

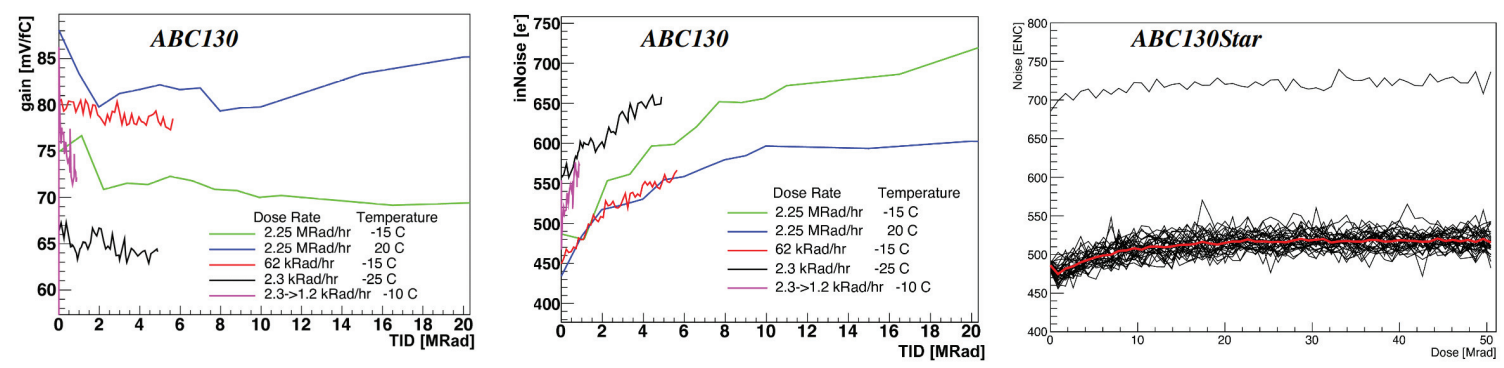

Figure 2.11: Signal to noise ratio consequences of TID in the ABC130 and ABC130Star. Left; ABC130 gain as a function of TID. Centre; ABC130 input noise as a function of TID. Right; input noise as a function of TID for the improved radiation hard design of the ABCStar. [1]

ITk cannot be entirely escaped. The projections of ionizing dose rate and TID, the latter of which was seen in Section 1.4.3, lead to the conclusion that not all components will reach the backside of the bump at the ten year mark. Coupling this to the realization that the effects of the TID bump will move outward from the high radiation areas over time has interesting implications for the powering and cooling of the ITk. A detailed operational plan tested through finite analysis simulation addresses these and sensor related concerns (Sections 3.4.3 and 4.3.1).

\subsubsection{The 2016 Testbeam Campaign and End-of-Lifetime Performance}

Complimentary to the sensor testing aspect of my Master's level research was my involvement in the two 2016 ITk Strip testbeams. Four and a half weeks of beam time saw the testing of six modules with a focus on irradiated devices. The main target was a full-size barrel module irradiated with protons to the $\sim 8 \times 10^{14} n_{\text {eqv }} \mathrm{cm}^{-2}$ ITklike fluence. A first for an ABC130 based device. Also measured was a comparative unirradiated counterpart, variously irradiated mini-sensor based modules, and an "Embedded Pitch Adapter" module (see Section 4.3.3). All goals set for the campaign were met providing many fundamental results that show the designs' readiness for high luminosity action, only a couple are discussed below.

At testbeam collimated, massive, charged particle beams of known energy are directed through the module. The individual beam particles, $\sim 5 \mathrm{GeV}$ electrons and $\sim 120 \mathrm{GeV}$ charged pions in this case, are tracked with extreme reliability by a six plane "telescope" system within which the device under test (DUT) is placed. This 


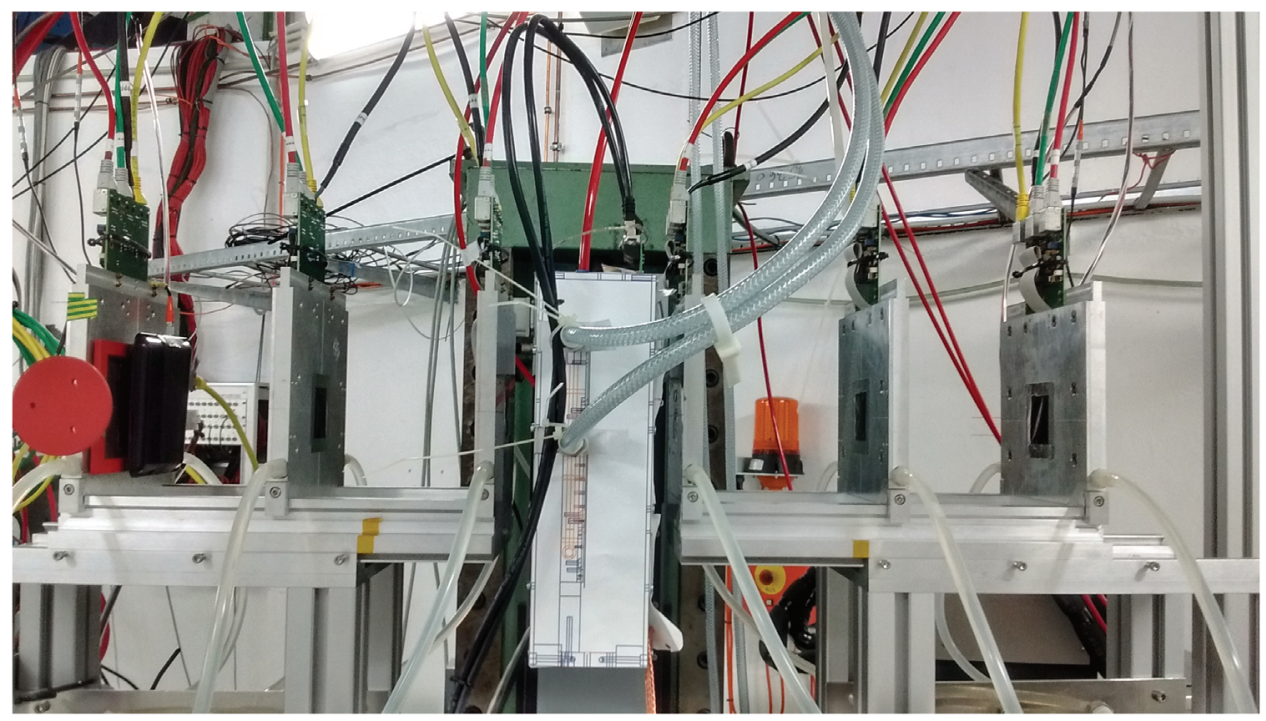

Figure 2.12: Photograph of the 2016 testbeam setup at DESY. The six planes of the EUDET telescope are seen on either side of the device under test in its air tight environmental box. The $5 \mathrm{GeV}$ electron beam enters from the right in this photo.

now conventional system is the EUDET telescope designed for R\&D of future colliders (under the EUDET collaboration). Shown for one of the two 2016 beams in Figure 2.12 , it features $3-4 \mu \mathrm{m}$ resolution in each of its six silicon pixel planes and a developed track reconstruction software (EuTelescope). Seen in the figure are the six planes, beam-line oriented, with three on either side of the environmental enclosure that the modules are tested in (to be able to simulate ITk thermal conditions, a necessity for the irradiated devices).

Testbeams are the main means to investigate the integral properties of the solid state tracking detectors such as the charge collection efficiency and the signal to noise ratio. This is because the high intensity beams offer the statistics and particle energies necessary for a realistic and meaningful assessment of the module's performance. Signal to noise is related to the sensor and readout. It has been mentioned in Section 1.5.1 and will be developed further here. The more purely sensor related charge collection efficiency quantity will be discussed in Section 3.3 and Section 3.3.3 in particular.

Constructed specifically for the critical R\&D operations of 2016 are the full-size pseudo-long-strip barrel modules denoted by the LS in Figures 2.13 and 2.14. These 


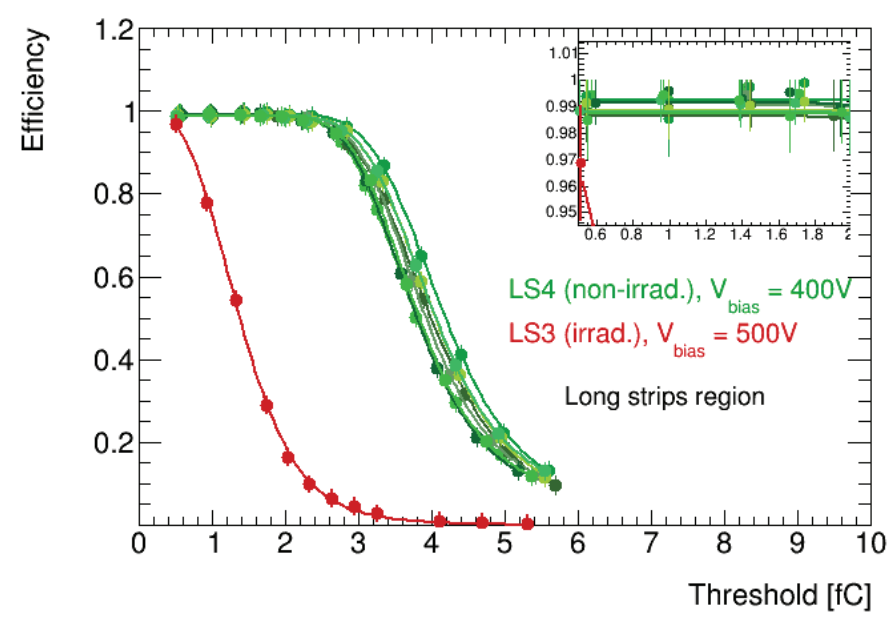

Figure 2.13: S-curve results for the irradiated and unirradiated full-size pseudo-long strip modules. Shown is the data for unirradiated LS4 (green) and irradiated LS3 (red) long strip modules. The green curves would show the same tail as the red curve if data had been collected. Results were obtained during the 2016 testbeam campaign. [1]

are obtained from short strip barrel sensors on which two of the strip rows have been wire-bonded together into a long strip proxy. The result is a hybrid barrel module with which the effects on both short (inner layer) and long (outer layer) strips can be deduced. LS3 is the irradiated module tested at biases of $500 \mathrm{~V}$ and $600 \mathrm{~V}$. LS4 is the identically constructed unirradiated module which was tested at $400 \mathrm{~V}$. Understanding the effect of bias as well as greater appreciation for these results can be gained in the following chapters and in Section 3.2 more specifically.

Figure 2.13 shows the familiar efficiency S-curve results for the long strips of the unirradiated LS4 (green) and irradiated LS3 (red) modules. Multiple curves are shown for LS4 corresponding to many groups of strips connected to different ABC130 since the beam width used is a little larger than the roughly $1 \mathrm{~cm}$ width of one $\mathrm{ABC}$ front-end chip. As expected the charge collection (Vt50) is significantly reduced by radiation damage (see Section 3.4).

The magnified view of the top left corner of the S-curves is shown even larger in Figure 2.14 to illustrate graphically the signal to noise requirements. The threshold scan results of data taken while the particle beams were 'turned off' has consequently been added. This yields the noise induced hit occupancy (noise occupancy) that is shown in red in Figure 2.14. The findings have been that greater than $99 \%$ hit 


\begin{tabular}{ccccccc}
$\begin{array}{c}\text { Module } \\
\text { Type }\end{array}$ & $\begin{array}{c}\text { Fluence } \\
\mathbf{1 0}^{\mathbf{1 4}} \mathbf{n}_{\mathbf{e q}} \mathbf{c m}^{-\mathbf{2}}\end{array}$ & $\begin{array}{c}\text { Charge } k e^{-} \\
\mathbf{5 0 0} \mathbf{~ V}\end{array}$ & $\begin{array}{c}\text { Charge } k e^{-} \\
\mathbf{7 0 0} \mathbf{~ V}\end{array}$ & Noise $e^{-}$ & $\begin{array}{c}\text { S/N } \\
\mathbf{5 0 0} \mathbf{~ V}\end{array}$ & $\begin{array}{c}\mathbf{S} / \mathbf{N} \\
\mathbf{7 0 0} \mathbf{~ V}\end{array}$ \\
\hline SS & 8.1 & 13.7 & 16.1 & 630 & 21.8 & 25.6 \\
LS & 4.1 & 17.3 & 19.5 & 750 & 23.1 & 26.0 \\
R0 & 12.3 & 11.5 & 14.0 & 650 & 17.7 & 21.5 \\
R1 & 10.1 & 12.5 & 15.0 & 640 & 19.6 & 23.4 \\
R2 & 8.7 & 13.3 & 15.7 & 660 & 20.3 & 23.9 \\
R3 & 8.0 & 13.8 & 16.2 & 640 & 21.4 & 25.1 \\
R4 & 6.8 & 14.6 & 17.0 & 800 & 18.4 & 21.3 \\
R5 & 6.0 & 15.3 & 17.6 & 840 & 18.3 & 21.1 \\
\hline
\end{tabular}

Table 2.5: Estimated signal-to-noise ratios for end-of-lifetime performance. All ITk Strip modules are shown. Fluence includes 1.5 safety factor. [1]

finding efficiency united with better than $0.01 \%$ noise occupancy is compatible with the $10: 1$ signal to noise target. These goals are shown as horizontal lines in black and red respectively.

The long strips of the unirradiated module are shown in the top plot of Figure 2.14 with the irradiated short and long strips at $500 \mathrm{~V}$ and $600 \mathrm{~V}$ bias potential shown in the bottom four. Even in the worst case it can be seen that the target signal to noise ratios have been met for small threshold intervals in the $0.5-0.6 \mathrm{fC}$ range. That is with the TID related noise increase of the ABC130 discussed in the previous subsection, the lower $500 \mathrm{~V}$ bias, and the use of inferior sensor prototype (low resistivity ATLAS12 sensors, see Section 4.2).

This result allows an estimate of the end-of-lifetime signal to noise ratio for all locations in the detector, this is shown in Table 2.5. The deficiencies in the current prototypes are adjusted for in the calculation. The improved noise profile of the ABCStar is known and given in the signal to noise plots and TID bump section above (Figure 2.11). It constitutes the first ingredient. Radiation hardness in more realistic, high resistivity sensors offered by other prototypes (ATLAS07) is the second. Its evidence can be seen in Figure 3.18. Detector response, studied, and operational conditions, already planned in detail, are all are that is left to allow the given projection results to be tabulated. Signal to noise ratios of about 20 to one are obtained leaving a wide margin for successful operation (greater or equal to ten to one).

My involvement in the 2016 campaign was fully integrated. This was ensured by the ATLAS author qualifying task of performing and documenting the new paradigm 

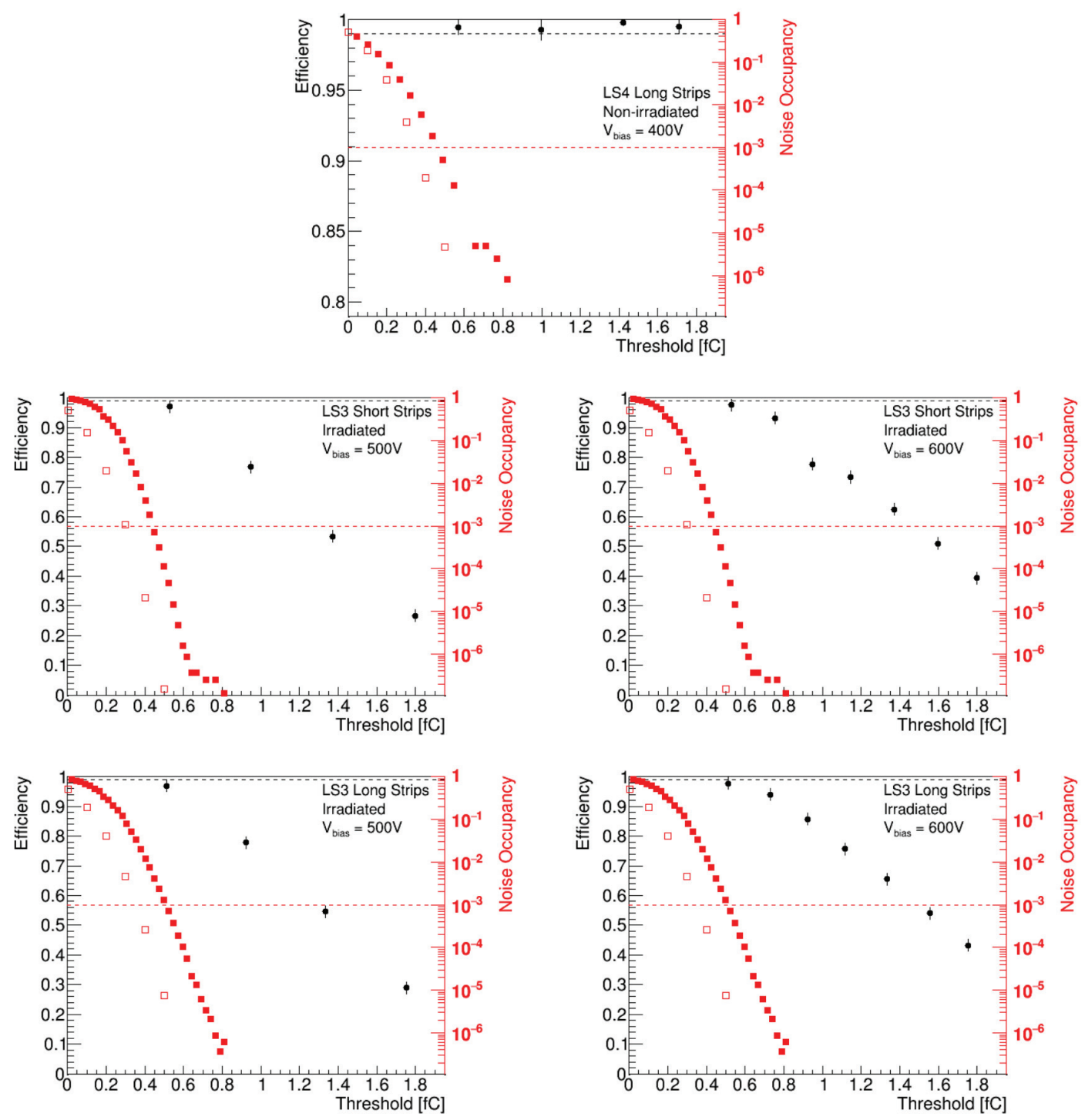

Figure 2.14: Proof that signal to noise requirements (dashed lines) will be met, given by early prototypes. Signal efficiency (black data) compared to noise occupancy as a function of readout chip threshold. Noise profiles of the ABC130 (filled red data) and ABCStar (unfilled red data) are given. Top; un-irradiated LS4 prototype long strips at 400 V . Centre row; irradiated LS3 prototype short strips at $500 \mathrm{~V}$ (left) and $600 \mathrm{~V}$ (right). Bottom row; LS3 long strips at $500 \mathrm{~V}$ (left) and $600 \mathrm{~V}$ (right). Test conditions and ATLAS12 prototype sensor quality are below ITk specification (see Section 4.2). [1] 
setting round of irradiation tests. In one respect I was part of a small three person team that debugged and conducted the first (in-lab) tests on LS3. The setup used was one tweaked and validated by myself. In the larger testbeam respect I partook in all the varied aspects of the testbeam experience.

It was an interesting, informative adventure with new challenges centred on several firsts for the ITk Strip collaboration's current (130nm-based) state. The first full-size irradiated module joined two other irradiated devices requiring new considerations in the cooling implemented in the setup. This constituted the main problem overcome in the first testbeam.

The second testbeam location was another first; utilizing the $120 \mathrm{GeV}$ charged pion beam produced in an off-shoot of the LHC's largest pre-accelerator the Super Proton Synchrotron (SPS) (see Figure 1.1). The newly irradiated full-size device and electronically noisy environment proved the main hurdles there with previous ITk Strip experience being built at DESY's incomparable $5 \mathrm{GeV}$ electron accelerator in Germany.

As the only collaborator with full involvement in both testbeams I was able to take a leading role in the second beam where the irradiated LS3 was tested for the first time. This, coupled with the qualification task directive of irradiated device testing mean that I was intimately connected with the critical successes demonstrated in Figures 2.13 and 2.14. 


\section{Chapter 3}

\section{The Physics of Silicon Microstrip Detectors}

This point dictates a change of gears. The focus of this Master's thesis is the ITk's unique silicon microstrip sensors. The in-depth exploration of the ITk Strip detector's most fundamental component starts in this chapter with a treatment of the physics that dictates its tracking potential.

The basics of semiconductors and how their sensitive electrical properties are exploited to allow micron position resolution is addressed in Sections 3.1 and 3.2. These basics allow a more developed picture of the microstrip silicon type of detector to be painted. Section 3.3 overviews the characteristics essential to safe operation of microstrip detectors with a focus on the ITk's unconventional $n^{+}-i n-p$ design. Finally, in Section 3.4 the effects of radiation damage in silicon sensors are discussed.

The unrivalled HL-LHC fluence projections have necessitated pioneering work in the field of radiation hard silicon. Advancements integral to the ability to operate in these conditions are discussed further in Chapter 4 while here (Section 3.4) the concentration is on the more general knowledge (mostly) known at the start of the HL-LHC R\&D. An overview of the ITk sensors follows naturally in Chapter 4 before its current testing is addressed in Chapter 5. A perusal of the basic ITk Strip design, offered in 4.1.1, can lend contextual significance to the physics discussed here. 


\subsection{Semiconductors and the P-N Junction}

Like a great deal of the semiconductor industry the basis of the solid state charged particle tracking detector is the p-n junction. This electrical interface between pdoped and n-doped semiconductor material dictates much of the physics at play in the ITk Strip sensor. [16, 17, 48]

As the name suggests semiconductors, such as silicon, have electrical properties that are intermediate to insulators and conductors. This transitional nature makes the semiconductors extremely sensitive to a large number of factors including temperature and the exact composition of the material. This ability to tune the electrical properties (for example by introducing a small dopant concentration) has led directly to the development of the electronics industry. The information age lives on silicon.

The semiconductor nature arises naturally in the band theories of solid state physics. In a crystalline solid with a periodic lattice the delocalization of the bound electrons' quantum mechanical wavefunctions is energetically favourable. Each discrete energy level of the lattice's constituent atom is split into a near continuum of levels dictated by the allowed energy eigenstates over the full lattice extent. These energy eigenstates are given by Bloch's theorem and reflect the periodicity of the structure. The bands are filled using Fermi-Dirac statistics (ie. up to the Fermi level) and it is the presence of open energy states thermally accessible to those filled by the electrons that dictates the conductivity of the material. $[16,17]$

This concept is summarized in Figure 3.1. Figure 3.1 (b) and (c) show the possible band structures of a metal. The valence band is either unfilled (c) or the valence and conduction band are overlapping in energy (b). In Figure 3.1 (a), a band gap exists between the highest filled level and lowest empty level (the Fermi energy is between the bands). Both insulators and (intrinsic) semiconductors have this feature and differ only by the size of gap (which is considered in comparison to thermal energies, $k T$, see Figure 3.2).

The case of the semiconductor is considered in more detail in Figure 3.2. $\mathrm{n}$ and $\mathrm{p}$ type semiconductors are well founded in band theory and are shown in the bottom two plots of this figure. The second column shows the density of states. The third column of plots represents the Fermi distribution of electron energy levels in each case (some small, non-zero temperature is used). The fourth shows the concentration of the majority charge carriers (electrons (n) and holes (p)) in the bands. 


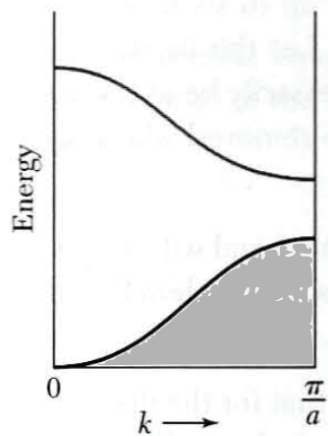

(a)

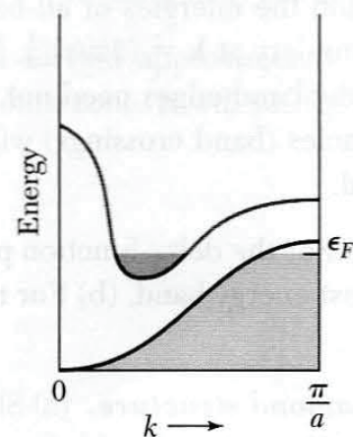

(b)

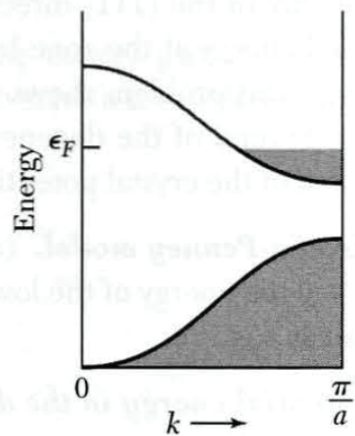

(c)

Figure 3.1: Band structure variants. Sub-figure (a) shows an insulator or intrinsic semiconductor (dependent upon the size of the band gap). The Fermi energy sits in the band gap. Sub-figures (b) and (c) show a metal with overlapping valence and conduction bands (b) and an unfilled valence band (c). [16]

$\mathrm{n}$ and $\mathrm{p}$ type materials are formed when an intrinsic semiconductor (eg. silicon) is evenly doped with small concentrations of specific impurities. These impurities are chosen to have energy levels in the band gap of the semiconductor but near either band. For n-type the impurity has an electron energy level just below the conduction band. Electrons are easily excited into conduction and these levels are called donor levels, $E_{D}$. As can be seen in Figure 3.2 this entails electrons as the majority charge carriers. For p-type, Figure 3.2 (c), the impurity's level is just above the valence band. Electrons are thermally excited into these "acceptor" levels, $E_{A}$, leaving behind vacancies in the lattice which act as quasiparticles called holes $\left(h^{+}\right)$. For p-type the majority charge carrier are the holes. In both cases strongly doped variants are conventionally denoted with a " $+" ; p^{+}$and $n^{+}$.

The integral physics of the p-n junction can now be understood. When brought into contact the excess majority charge carriers (electrons and holes) offered by the shallow (donor and acceptor) levels in the band gaps of the $\mathrm{n}$ and $\mathrm{p}$ type (respectively) experience Coulombic attraction. They migrate across the interface in opposite directions recombining as they go. Since the two semiconductors are initially neutral this initiates the formation of immobile space charge (of opposite sign in the two materials, the left behind dopant ions). This space charge sets up a 'built-in' potential which counteracts and arrives at equilibrium with the now thermally powered migration of carriers across the interface. The $\mathrm{p}-\mathrm{n}$ junction at thermal equilibrium is shown in Figure 3.3 for a junction with an abrupt dopant profile (left) and a gradual dopant 

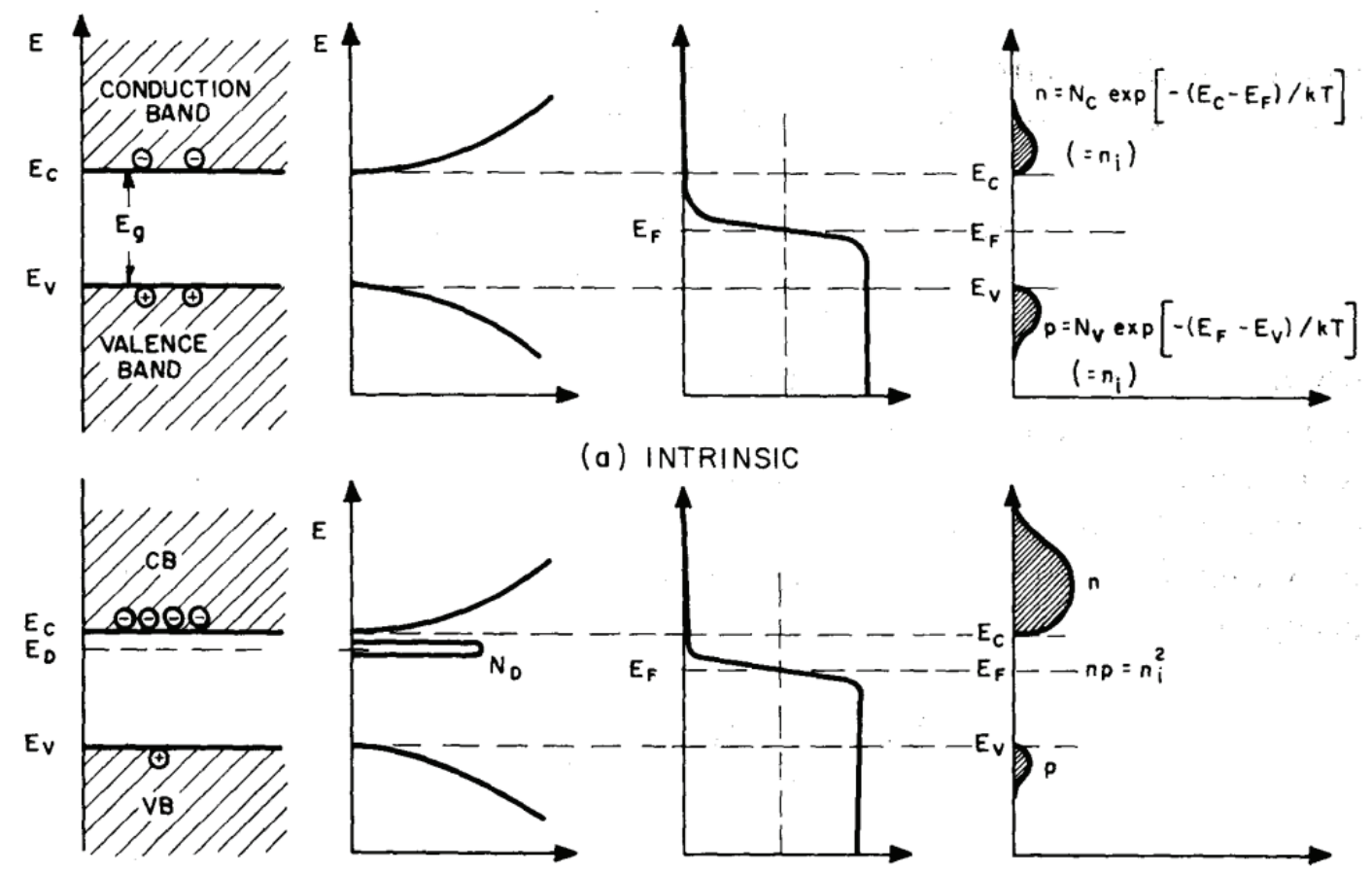

(b) $n$ TYPE
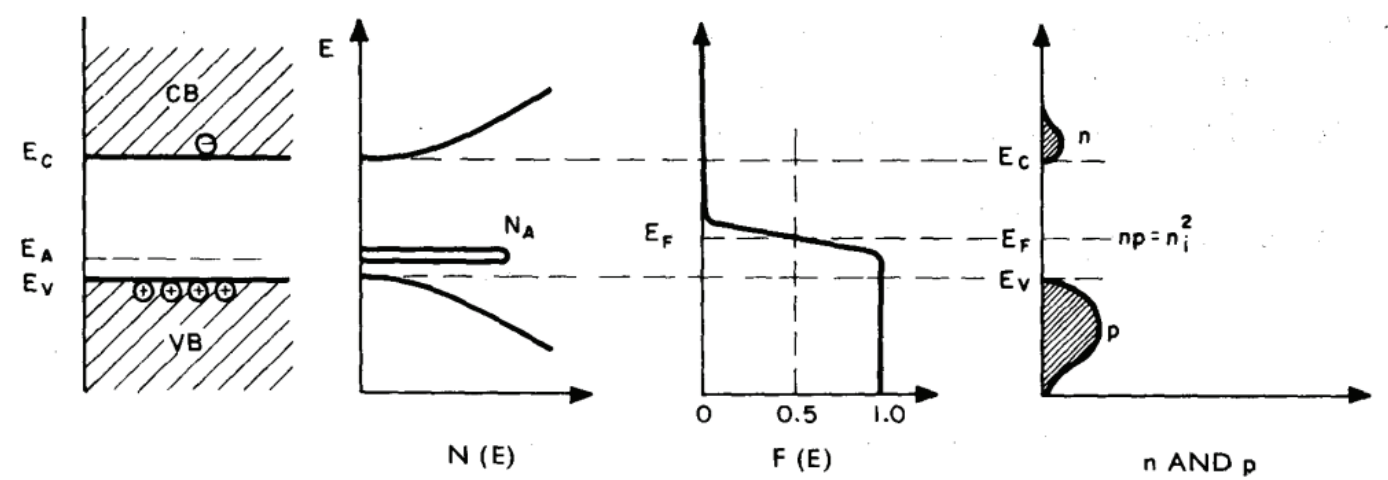

(c) P TYPE

Figure 3.2: Schematics depicting the band structure, density of states, Fermi-Dirac distribution of energy levels (for a non-zero temperature), and the charge carrier concentrations for (a) intrinsic, (b) n-type, and (c) p-type semiconductors. [17] 

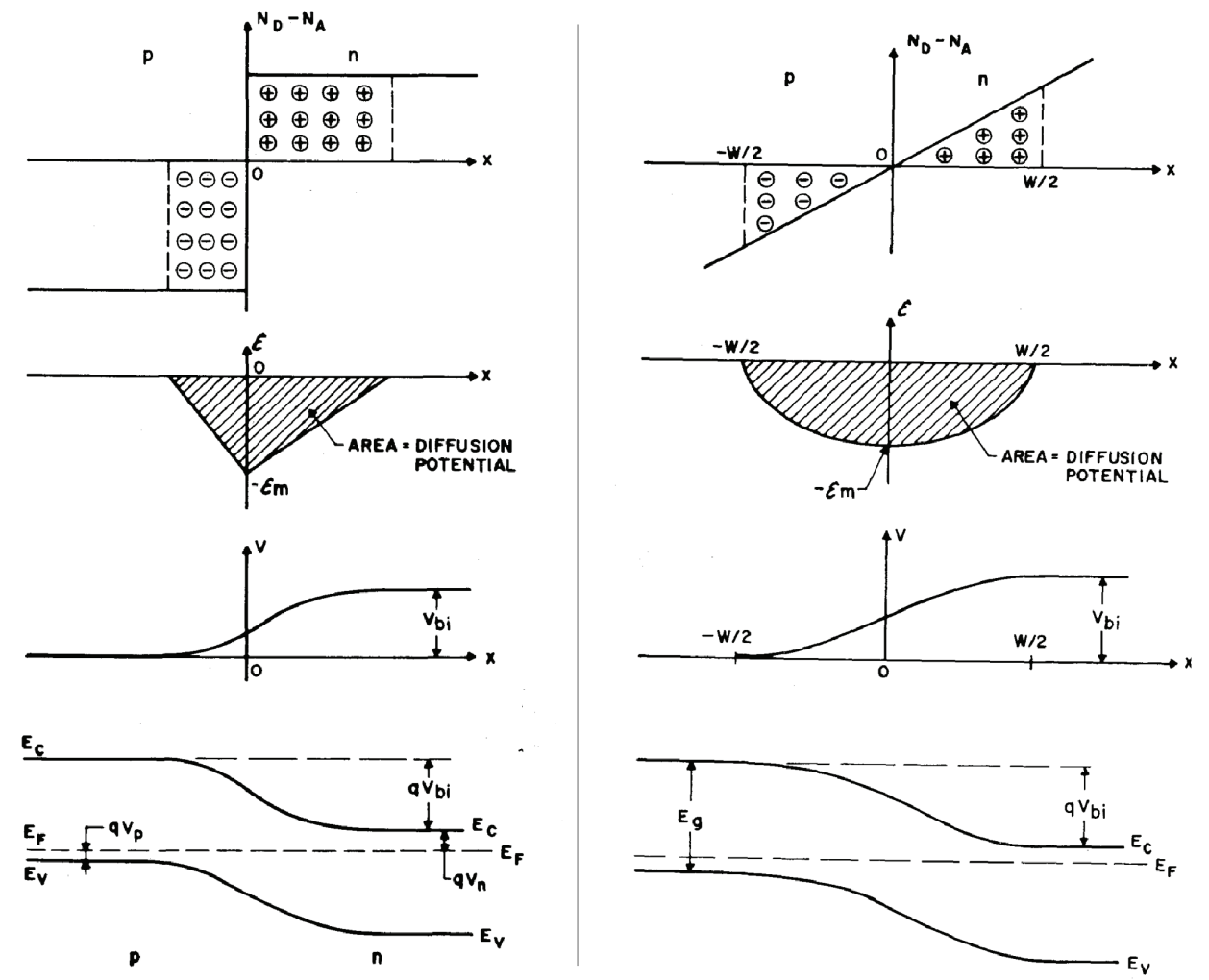

Figure 3.3: The p-n junction in thermal equilibrium for a junction with an abrupt dopant profile (left) and a gradual dopant profile (right). From top to bottom are the impurity distributions, field distributions, electric potentials, and energy band diagrams across the interface. [17]

profile (right). At room-like temperatures this potential is $O(1 \mathrm{~V})$ [49].

At equilibrium, the central space charge region over which the small built-in voltage acts is devoid of mobile charge carriers and is called the depletion zone. The size of this region can be expected to change if a voltage is applied across the junction. When the applied voltage is oriented against the built-in voltage the depletion zone disappears and the flow of majority charge carriers results in high conductivity. This is called forward bias. In the opposite case, the applied voltage reinforces the built-in voltage and the depletion zone expands. In this case the junction is in a reverse bias state (in the text this case will be assumed unless otherwise specified). The current flow is small since only the minority concentration of charge carriers have Coulombic reason to cross the junction (due to the immobile space charge). This is the electronically useful diode nature of a planar p-n junction. Figure 3.4 shows 


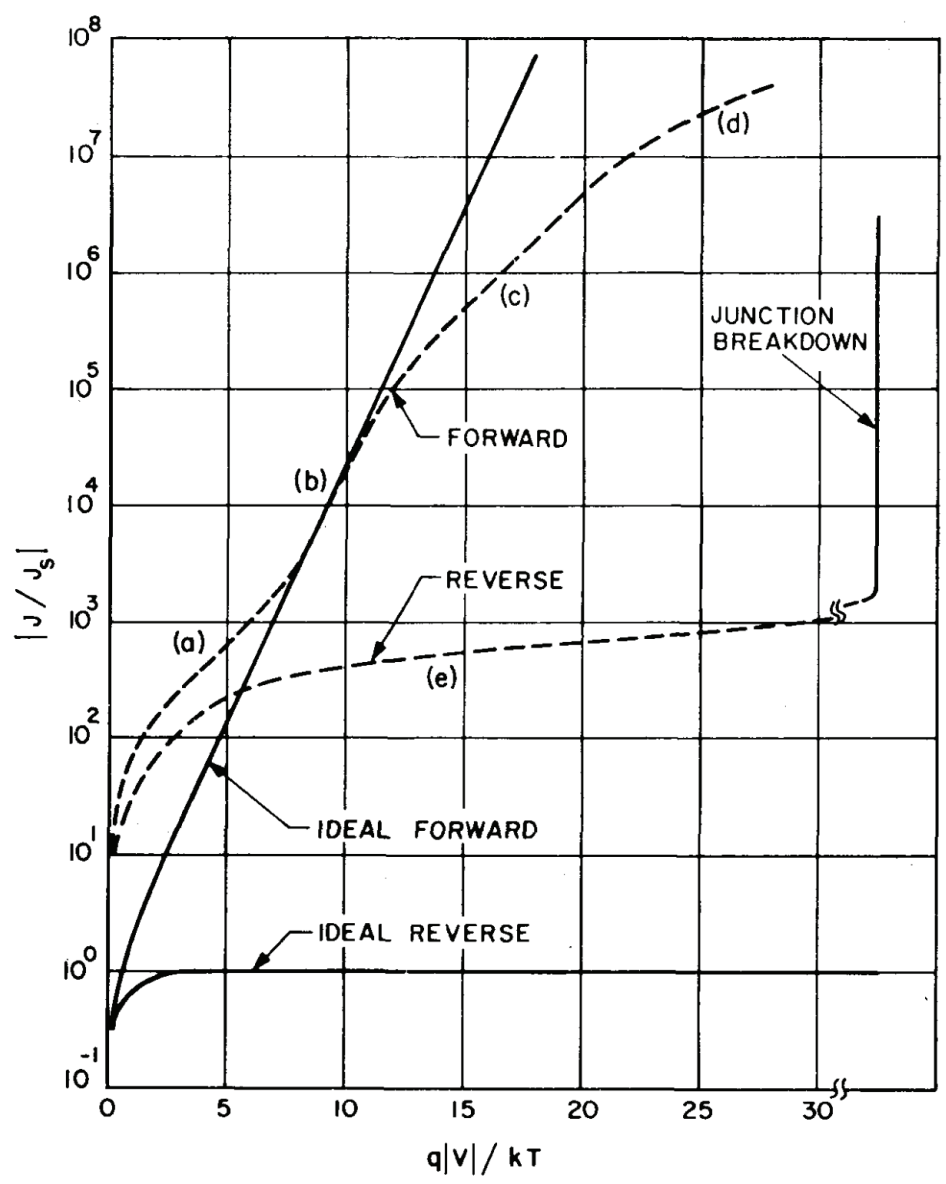

Figure 3.4: IV curve of a realistic silicon (dashed line) and ideal (solid line) diode. Current is normalized to the ideal reverse saturation value. Aspects of the reverse bias curve are discussed in the Sections 3.3 and 3.4. [17]

the current-voltage (IV) character of a realistic silicon (dashed line) and ideal (solid line) diode. The discrepancy between the two is discussed further in Sections 3.3 and 3.4. $[17,48,49]$

\subsection{Microstrip Semiconductor Detector; Principle of Operation}

The key to the p-n junction's tracking potential is the depletion region and it's diode character. A charged particle traversing a material will cause ionization along its path as it interacts electromagnetically with the nearby medium (the amount of 


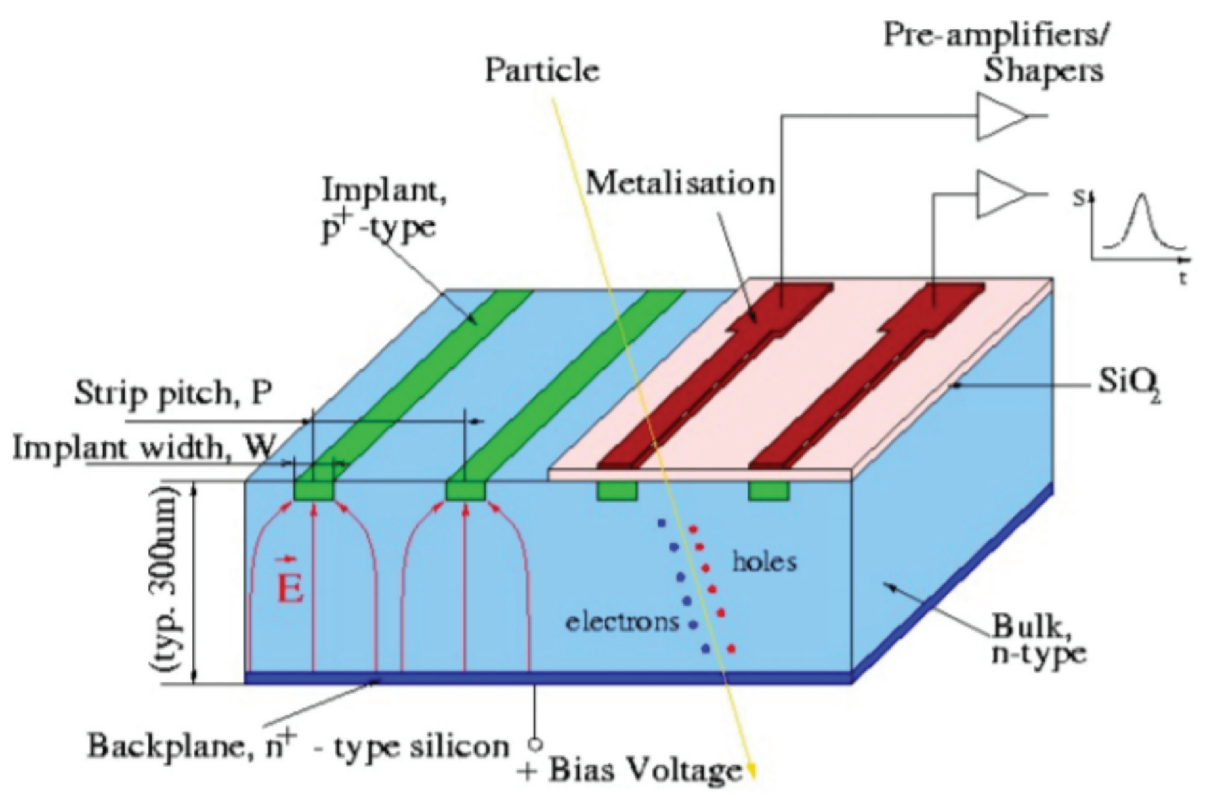

Figure 3.5: Schematic representation of a semiconductor microstrip detector. Shown is a single-sided, $p^{+} i n n, \mathrm{AC}$ coupled, silicon detector. This design is similar in base operation to the ITk Strip sensor but with the types reversed. [18]

which is dictated by the Bethe formulae and the material). In the depleted zone of a semiconductor the liberated $e^{-}$and $h^{+}$are the only mobile charges. After liberation they are swept by the space charge field to the two electrodes and the depletion area is maintained. A voltage pulse is induced that is proportional to the magnitude of moving charge liberated by the transient charged particle. Fundamentally, in the depleted zone with a strong field, the charge is transversely localized to the track of the transient. Intuitively, it is crucial to maximize the volume of the depletion zone to allow more charge to be liberated and a larger signal to be obtained. [17,48,49]

The basic principle of the AC-coupled microstrip semiconductor detector is shown in Figure 3.5 (for a single-sided $p^{+}-i n-n$ detector). It begins with a flat, ultra-pure bulk substrate of either $\mathrm{p}$ or $\mathrm{n}$ type semiconductor with a relatively low dopant concentration. On one or both of the flat sides are patterned micron-scale implants of strongly opposite doping at a regular micron-scale spacing (1-D strips or 2-D pixels). These readout implants do not extend very deep in comparison to the detector thickness (which necessitates the relative doping levels as will be discussed in Section 3.3). An array of p-n junctions results from this mating. These are connected in parallel and biased in the reverse state to expand the depletion region. Ideally, for sensitivity, 


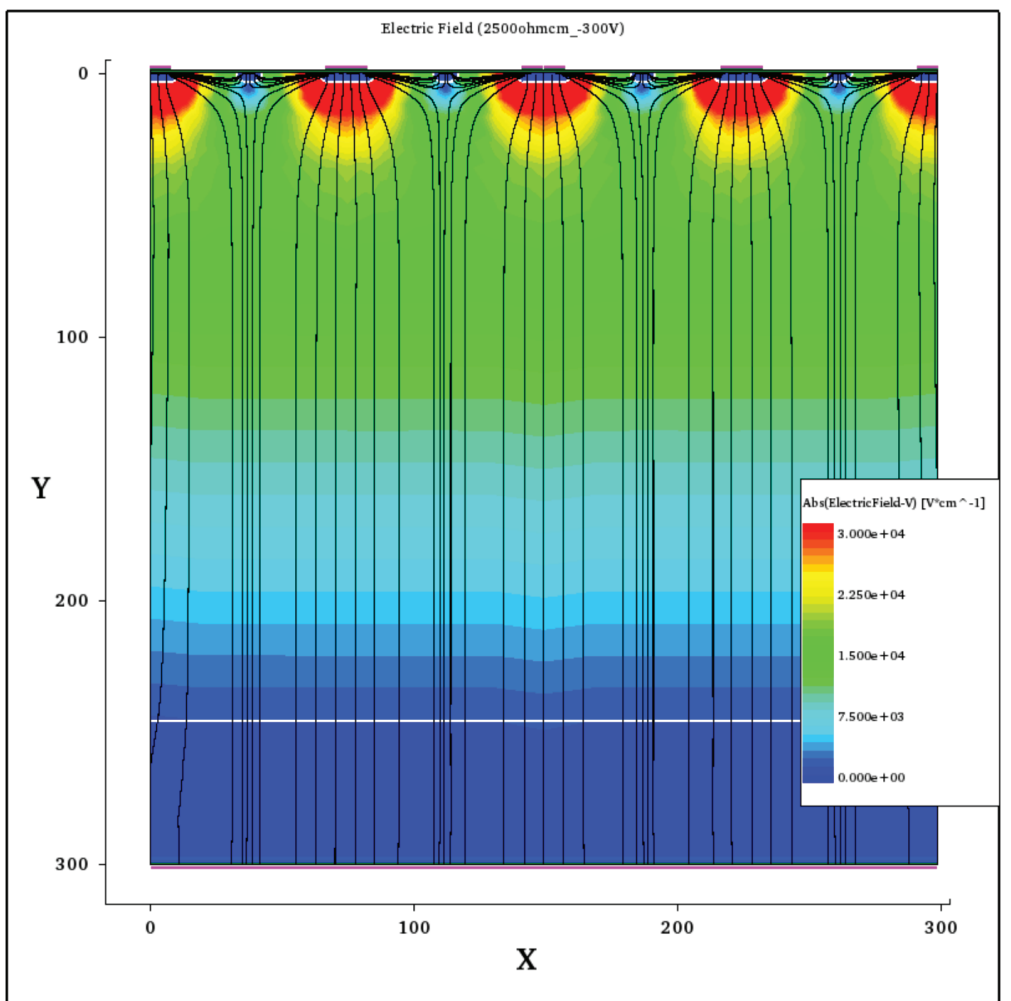

Figure 3.6: Simulation of electric field strength in a silicon microstrip detector at partial depletion (white line). [19]

the reverse bias voltage is such that the depletion region expands from the junction across the entire bulk of the detector. The electric field in the vast bulk of the sensor is then quite vertical and any tracking particle will produced a localized cluster with minimal transverse spread. The liberated charge flows along the field lines to very few readout implants since the field is quite well contained in the individual cells of the strip array (see Figure 3.6). The voltage pulse induced is thus strip localized and a position measurement with a resolution related to the strip pitch is obtained. In fact, the obtainable resolution is a factor of $\sqrt{12}$ less than the strip pitch exploiting the mechanism of charge spreading by using a centroid calculation. [17,48,49] 


\subsection{Characteristics of Microstrip Semiconductor Detectors}

In this section the focus will be on phenomena that are applicable to the ITk operational conditions, reverse bias of a single sided, AC-coupled, $n^{+}-i n-p$ detector. In sensors of this type the p-n junction determines the bulk physics while the dielectric layer of the coupling capacitor plays the leading role in the surface realm. Both must be considered to understand the mechanisms of charge sharing, charge losses, and breakdown important to successful operation in a tracking capacity.

\subsubsection{Depletion Capacitance}

The strip surface region does not extend far in z compared to the full thickness of the sensor and the bulk physics can be approximated as arising from a planar p-n junction. This is advantageous since it means that the electric field in the bulk of the sensor is extremely vertical. However, since the material is originally neutral, equal space charge must exist on either side of the junction in the depletion zone. Thus by necessity the small feature must be of considerably higher dopant concentration than the bulk to help push the extent of the depletion zone (refer Equation 3.1). [17,49]

The relation between the depletion depth and the voltage across the junction is derived by first considering the thermal equilibrium case (Figure 3.3). The steadystate condition is exploited requiring that the space charge be equal on either side of the junction and that the field vanish at the zone boundaries. Importantly, since the space charges are immobile, this consideration implies that the depth of the depletion zone on either side of the junction is inversely proportional to its dopant concentration. Poisson's equation is then used to relate the electric potential with the charge distribution and therefore depletion depth (see Figure 3.3). This approach is of course valid to the steady state case of a constant reverse voltage if the built-in voltage is replaced by the reverse bias voltage (built-in voltage plus applied). [17,49]

Equation 3.1 shows the resulting relation with d the depletion depth, $\epsilon_{s c}$ the dielectric permittivity of the semiconductor material, $\mathrm{q}$ the elementary charge, $\mathrm{V}$ the reverse bias voltage, and $N_{A / D}$ the doping concentrations in the $\mathrm{p} / \mathrm{n}$ sides. The

presence of the reduced or effective doping concentration $\left(\frac{1}{N_{e f f}}=\frac{1}{N_{A}}+\frac{1}{N_{D}}\right)$ relates the fact that the depletion extends unequally in either side of the junction. $[17,49]$ 


$$
d^{2}=\frac{2 \epsilon_{s c}}{q}\left(\frac{1}{N_{A}}+\frac{1}{N_{D}}\right) V=\frac{2 \epsilon_{s c}}{q N_{e f f}} V
$$

An important consideration for a realistic detector is illuminated when the resistivity of the doped semiconductor is subbed into the depletion voltage relation. Since the current flow in these materials under normal conditions is dominated by the majority charge carriers the resistivity, $\rho$, is defined by their mobility, $\mu$ and concentration, $\mathrm{N}$.

$$
\rho_{\text {doped }}=\frac{1}{q \mu_{\text {maj.carrier }} N_{\text {maj.carrier }}}
$$

Combining equations 3.1 and 3.2, yields 3.3 (where notation follows the example that $h^{+}$inp refers to the hole majority carriers in p-type silicon). Thus for the best sensitivity (maximum depletion) high resistivity semiconductor material is desired. As will be seen in Section 3.4 impurities reduce the resistivity and since they must be added for operation the starting material contamination must be absolutely minimized. Thus, very high purity semiconductor is required for optimal performance of a microstrip detector.

$$
d^{2}=2 \epsilon_{s c}\left(\rho_{p} \mu_{h^{+} i n p}+\rho_{p} \mu_{h^{+} i n p}\right) V
$$

From the discussion of $\mathrm{p}-\mathrm{n}$ junctions it is clear that the depleted volume acts similar to a charged parallel plate capacitor. As the bias voltage is increased the depth of the immobile space charge region increases and thus the capacitance decreases. The relation between the depletion layer capacitance (per unit area), C, and the reverse voltage follows from the parallel plate-like nature and Equation 3.1. It is given in Equation 3.4.

$$
C=\frac{\epsilon_{s c}}{d}=\sqrt{\frac{q \epsilon_{s c} N_{e f f}}{2 V}} \Rightarrow \frac{1}{C^{2}}=\frac{2}{q \epsilon_{s c} N_{e f f}} V
$$

\subsubsection{Full Depletion Voltage}

The question remains on how best to standardize quantification of the all-important depletion performance in the case of large production where thickness, impurity concentrations and resistivity may vary from batch to batch. An obvious choice exists in considerations for operation of the detector; the full depletion voltage, $V_{f d}$. This 


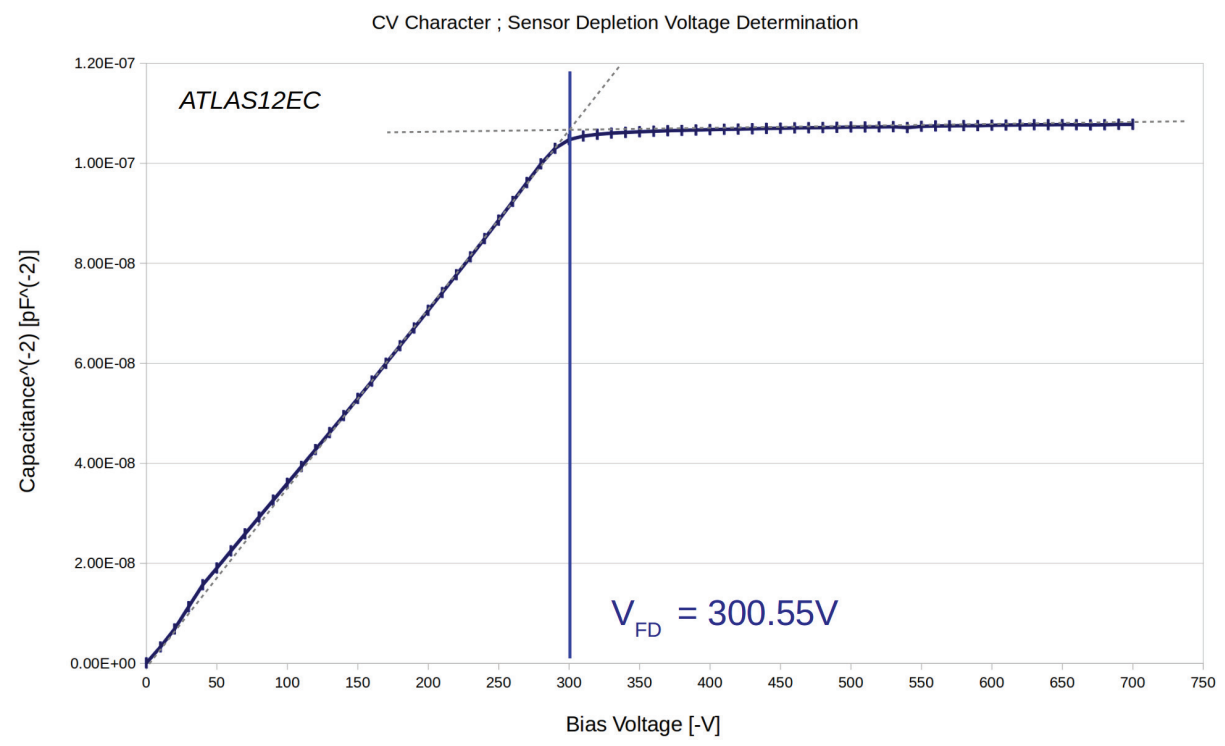

Figure 3.7: The capacitance-voltage relationship for a microstrip detector biased past the full depletion voltage. The full depletion voltage is easily identifiable from the intersection of the depleting and depleted linear trends. The data shown was collected with the most recent ATLAS ITk Strip prototype (ATLAS12EC) at Carleton University.

is the minimum reverse bias potential at which the full bulk of the detector is depleted. It thus designates the minimum bias at which the sensor will operate with full sensitivity. Keeping this value low by using high purity material with tuned doping concentrations is very important since it dictates the power consumption for optimal performance and will increase with radiation (see Section 3.4). Microstrip detectors operating with reverse bias voltage less (greater) than the $V_{f d}$ are said to be operating in partially (fully) depleted mode.

The conventional method for experimentally determining the full depletion voltage is the capacitance voltage (CV) curve. A typical CV curve is shown in Figure 3.7 using data obtained in the Carleton lab (see Chapter 5). The full depletion voltage, $V_{f d}$, is shown. The linear rise up to $V_{f d}$ is due to the advancement of the depletion zone across the bulk (Equations 3.4 and 3.1). The almost flat plateau for voltages greater than $V_{f d}$ signifies the fact that the sensor cannot be depleted further. This plateau would be flat in an ideal case with the slight rise caused by over-depletion as the electric field strength climbs (it bows out at the edges of the sensor) [50]. [17,48,49] 


\subsubsection{Charge Collection Efficiency}

While the potential for charge liberation, and therefore signal, is defined by the size of the depletion region further factors affect how much charge actually makes it to the collecting electrodes. The concept of charge collection efficiency (CCE) arises in the true case of an unideal material. It is defined as the ratio of charge collected on the electrode to total free charge liberated by a passing particle. The main mechanism that contributes to a reduction in the charge collection is "trapping" of the charge carriers by undesired impurities. For this to occur the abnormality must have electron energy levels in the band gap. For charge collection losses the contaminants involved are deep traps; the energy required for the trapped carrier to be promoted to its conduction band is large compared to the available thermal energy. If the charge carrier, which has only been liberated due to the deposited track energy, falls into this trap it is statistically unlikely to get thermally excited to the adjacent bands. It is important to note that impurities also refers to impurities in the lattice structure (defects) as long as the deep traps are present. As can be intuitively understood the rate of trapping is dependent upon the mobility of the charge carriers and the use of higher electric fields (by applying $V>V_{f d}$ ) can reduce the loss in efficiency. Trapping and impurities will be discussed further in Section 3.4. Charge collection efficiency is of obvious importance to the detector performance and entails the use of ultra-pure, mono-crystalline substrate. [17, 28, 48, 49,51]

It is useful to clearly note that the depth of a trapping level refers to the energy cost of releasing the attracted carrier from its grasp (the depth of the potential well). The available thermal energy, $k T$, is used as a yard-marker. The depth is related to the energy level's distance from the band gaps. A positive trap with an energy level near the valence band energy will be deep (trapping $e^{-}$) while a negative trap with the same energy level will be shallow (trapping $h^{+}$). Importantly, if an impurity is large of charge (chemical impurity) or large of size (lattice defects) the depth of the trap can increase dramatically. Intuitively, trap depth and the average length of time spent in the trap by the carrier (inverse of trap lifetime) are related. Finally, it can be noted that not all defects are necessarily electrically active. Although only electrically active defects participate in trapping, inactive traps may transmute or coalesce into active traps spontaneously (see Section 3.4.3). 


\subsubsection{Leakage Current}

The physics of the reverse leakage current through the depletion zone has already been outlined (see Figure 3.4). The current can only be substantiated by the minority charge carriers since the majority carriers cannot cross the junction. The result is a small saturating current. This is the ideal case (Figure 3.4) in reality however several more mechanisms contribute to the reverse leakage. Large among these are the thermal generation of $e^{-} h^{+}$pairs and surface leakage. The latter of these arises over the surface bound depletion zone (between bias rail and edge metal) and has a complex relation on the encapsulation, humidity, contamination, and edge structure. It is discussed more in Section 3.3.6. The former dominates [17] and lends to the detectors a strong temperature dependence.

The thermal generation of charge is facilitated by impurities with energy levels in the band gap. These are the aforementioned trapping centres since at a constant temperature thermal equilibrium of the shallow dopant levels has already been exceeded by the applied bias. The current generated is related to the charge of the carrier and the volume from which it comes (the depletion thickness). The temperature dependence enters in with the generation rate which is proportional to the carriers available for promotion and lifetimes of the carriers in the traps. The charge carriers available come from the states of the base material (the intrinsic semiconductor); as discussed, this concentration, $n_{i}$ is found from the filling of states according to Fermi-Dirac statistics. For a three dimensional solid one finds Equation 3.5 where $\mathrm{k}$ is Boltzmann's constant and $E_{g}$ is the band gap energy. [17,49,52]

$$
n_{i}(T) \propto T^{3 / 2} e^{\frac{-E g}{2 k T}}
$$

The temperature dependence of the generation lifetime, $\tau_{g e n}$, is more complicated but can be summarized as $\tau_{g e n} \propto T^{-1 / 2} e^{\frac{\Delta}{k T}}$ where $\Delta$ is the difference between the trap level and the intrinsic Fermi level [52]. With the reasonable assumption that the trapping is equally effective for both electrons and holes, the generation lifetime is minimized when the trap is closest to the intrinsic Fermi energy (putting it equidistant from the bands). In other words when $\Delta$ is zero and the exponential term is as it appears in Equation 3.5. Now the band gap energy temperature dependence can be incorporated, for silicon in the range $250 K-415 K$ it is given by $E_{g}=E_{\text {const. }}+\alpha T$ with $E_{\text {const. }}=1.206 \mathrm{eV}$ and $\alpha=2.73 \times 10^{-4} \mathrm{eV} / K$ [52]. Since the mid-gap trap 
generates the majority of the thermal current the current temperature relation will be well approximated by Equation 3.6. [17,49,52]

$$
I_{\text {leakage }}(T) \propto T^{2} e^{\frac{-1.21 e V}{2 k T}}
$$

\subsubsection{Junction Breakdown}

If the increase of reverse bias field is continued beyond full depletion the electric field in the depleted bulk will build until the liberated charges travelling to the electrode gain enough kinetic energy due to the field to cause secondary ionization. If the charges are not slowed by some mechanism then avalanche multiplication occurs resulting in large current flow across the junction. This renders the detector inoperable since the depletion zone's electric field is broken. The applied voltage at which this breakdown occurs is called the breakdown voltage and is related to the maximum electric field strength and the width of depletion. The maximum electric field strength in the detector is affected by a number things such as doping concentration and uniformity, impurities, and geometry. The temperature dependence of the avalanche multiplication breakdown is positive; the breakdown voltage increases with increasing temperature. This can be understood from the decrease in the electron-phonon mean free path as the lattice vibrations increase with temperature. [17, 21, 38, 49, 53, 54]

The detrimental feedback loop of the normal sensor operation must be addressed. The bias resistors which must be large valued to isolate the strips from one another mean that there will be non-negligible resistive heating despite the small current. The heat generated will cause the current to rise (Equation 3.6) which will cause more heat to be produced. This feedback loop, termed thermal runaway, may lead to the eventual breakdown of the sensor if the temperature is not controlled at a level congruent with the stable leakage current. [17,49]

Other mechanisms of breakdown exist. One such method involves tunneling of the quantum wavefunctions through a finite potential barrier (quantum tunneling) of the charge carriers between the bands. This effect is prevalent in junctions with both sides strongly doped. The tunneling probability is inversely related to the band gap energy, which decreases with increasing temperature. Thus the tunneling initiated breakdown voltage has a negative temperature dependence. [17,49]

Another associated phenomena are the microdischarge and microplasma effects. In these cases the breakdown is localized to a small high field region. The leakage 
current will have a sharp increase to a higher plateau as the current from the localized breakdown contributes (see Figure 3.13). These effects do not necessarily cause breakdown of the junction (rendering the detector unusable) but will obviously contribute. The difference between the two effects is where they happen; the surface for microdischarge and the bulk (large impurity sites) for microplasma. Interestingly, microdischarge is accompanied by photon emission in the infrared range (see Section 4.3.3). [17, 21, 29, 38, 49, 53-5.5]

\subsubsection{Surface Physics}

The surface physics of the AC-coupled microstrip detector revolves around the silicon dioxide layer and its interface with the semiconductor. Here the build up of charges and trapping states has two effects; the alteration of the field in the oxide with consequences for the signal integrity and the possible introduction of parasitic pathways (between strips or over the top-side edge bias-gap) with consequences for operability.

\section{The MOS Structure}

At the sensor surface the $\mathrm{Al}$ strip, $\mathrm{SiO}_{2}$, and $n^{+}$implant layers function as a metaloxide-semiconductor (MOS) structure. Thus to understand the surface effects a quick introduction to the MOS structure is needed. On the left side of Figure 3.8 is a schematic of the MOS geometry. On the right is the energy band diagrams without bias; in this case the work function in the metal is equivalent to the Fermi energy of the (doped) semiconductor on the other side. [17]

Application of voltage across the MOS results in three different phenomena dependent upon the potential's sign and magnitude. These are summarized in Figure 3.9; at left the band diagrams and at right the evolution of the surface charge, $Q_{s}$, as a function of surface potential, $\psi_{s}$ showing clearly the three regimes. For the $n^{+}-i n-p$, AC-coupled microstrip detector the voltage sign during operation is that of the depletion and inversion case (Figure 3.9). The result is the build-up of minority charge carriers on the semiconductor side of the interface. Eventually, a full layer of minority charge carriers will accumulate, the so-called inversion layer. This has dire implications for the inter-strip region of a microstrip detector since operation hinges on collection of minority carriers. This layer then provides the means for parasitic pathways between the strip implants or across the edge bias gap inducing undesired 

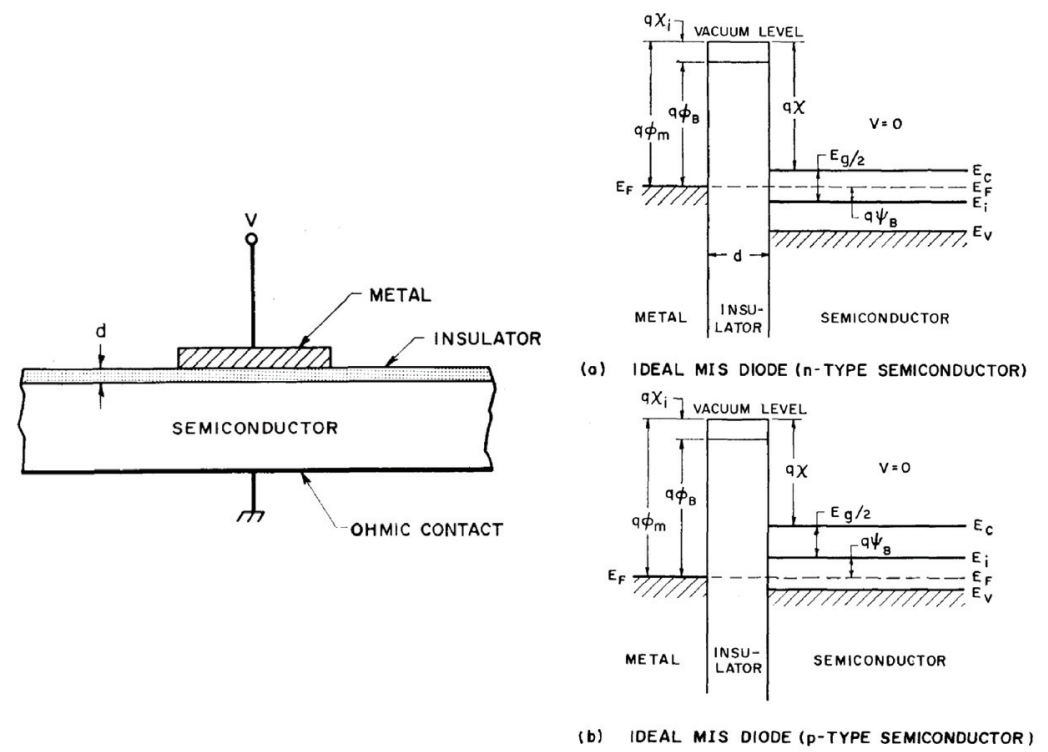

Figure 3.8: The metal-oxide-semiconductor (MOS) structure. At left, a simple schematic of the geometry. At right, the band diagrams at $V=0$ in the cases of $\mathrm{n}$ (top) and $\mathrm{p}$ (bottom). $\chi$ is electron affinity of insulator (i) and semiconductor, $\psi_{B}$ the potential barrier, $\psi_{m}$ the metal work function and $\phi_{B}$ the difference between the Fermi energy and its intrinsic counterpart. [17]

charge sharing (interstrip leakage current increase) and bulk leakage current increase. The charge sharing effect can be mitigated by using small amounts of more strongly doped bulk material between the strips (since it will have less minority carriers). Two main geometries of this isolation structure exist; the stronger dopant is applied in a thin layer across the interstrip region (eg. p-spray) or in thin traces parallel to the strips (eg. p-stop). [17,56,57]

\section{Realistic Surface Effects}

The above scenario is an ideal case assuming perfect boundaries and material with no charges in the insulator. In reality the situation is complicated by the trapping impurities and other effects which alter the inversion layer. Four main distinctions can be made; trapping states in the oxide bulk (ionized traps), trapping states at the interface, locked in charge at the interface, and mobile ion contamination. These are shown schematically in Figure 3.10 for a silicon - silicon dioxide interface. Another contributing effect through its interplay with the oxide's mobile ions is the variably 

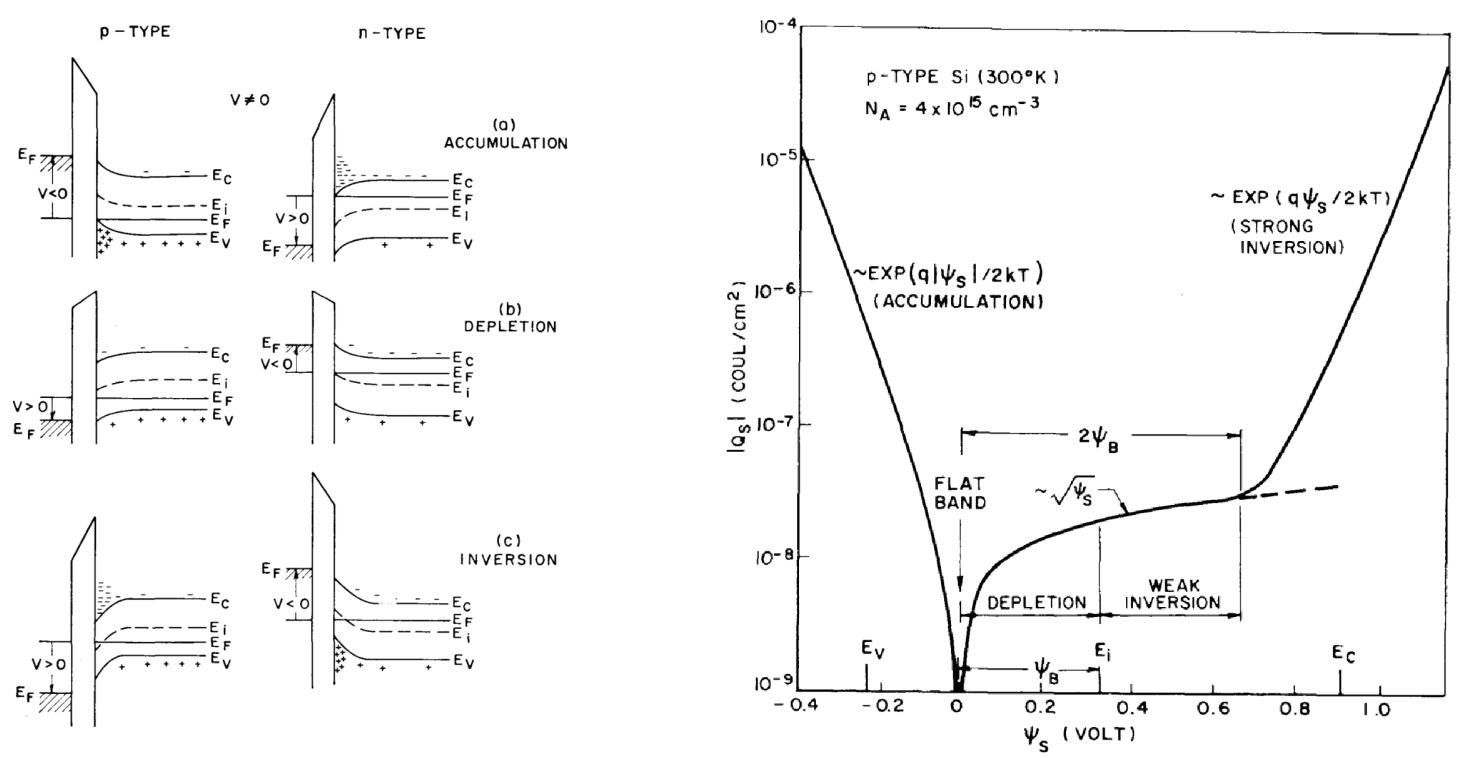

Figure 3.9: The three regime operation of a MOS under bias. At left, the band diagram representation of the three regimes of accumulation, depletion, and inversion for both $\mathrm{p}$ and $\mathrm{n}$ type semiconductor. At right, the evolution of surface charge, $Q_{S}$ as a function of surface potential, $\psi_{S}$ for a p-type sample with dopant concentration of $4 \times 10^{15} \mathrm{~cm}^{-3} \cdot \psi_{B}$ is the difference between the doped and intrinsic Fermi level. [17] 


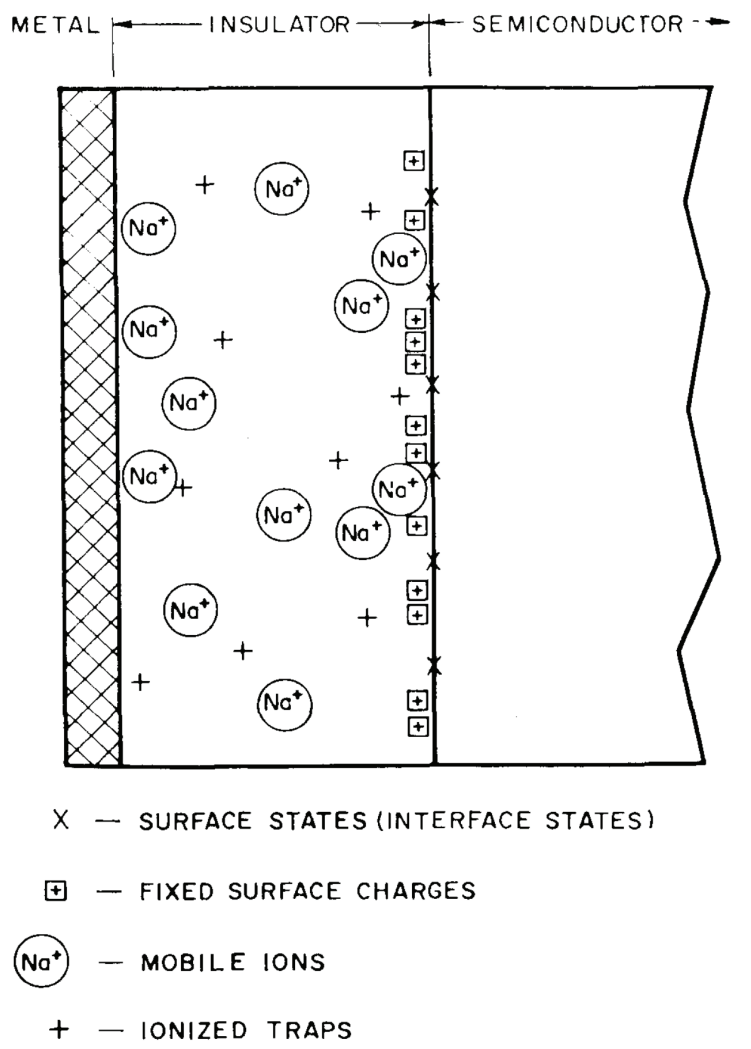

Figure 3.10: Schematic depiction of the classes of charges and states in an unideal MOS device. Not shown is the charge that accumulates on the outer passivation layer. [17] 
mobile charge on the outer surface of the passivation layer between the strips.

Trapping impurities (impurities with energy levels in the band-gap) in the oxide itself are due entirely to chemical centres (ions, as opposed to mechanical, lattice centres) since the $\mathrm{SiO}_{2}$ is amorphous. These traps can be built-into the oxide during manufacturing or induced by ionizing radiation (ionized traps). If these traps are near the $\mathrm{Si}-\mathrm{SiO}_{2}$ interface they will exchange charges with the silicon, otherwise they just affect the capacitive reaction of the MOS structure. At the interface, these ionic traps may be present but it is the lattice defect impurity that is overwhelmingly dominant. This is of course because the interface is, by definition, the transition between two compounds with different microscopic structures. If the interface traps are deep in nature they lead to locked in surface charge. If shallow the interface traps facilitate charge exchange between the materials. Importantly, interface states also exist at the boundary of oxide and air, offering a method by which contaminants can be leeched into the sensor if sufficient encapsulation is not applied.

The use of silicon dioxide, the natural oxide of silicon, is conventional. This is not only because it can be thermally grown but because it offers one of the smoothest possible interfaces (again for silicon) [20]. An example (there are a few different classes) of a silicon, silicon oxide interface is shown in Figure 3.11. Large lattice vacancies are seen, defining the interface layer and contributing to deep surface traps. In $\mathrm{Si}-\mathrm{SiO}_{2}$ interfaces it is well known that the surface accrues a locked in positive charge. Since it is lattice related the magnitude of this charge can vary quite strongly with the structure and quality of the interface. Figure 3.12 shows the measured surface state densities for three silicon crystal orientations; the excess in positively charged deep electron traps is evident for the (111) case. Unfortunately the unavoidable interface charge will reinforce the undesirable inversion layer since the positive charge build up induces a mirror charge in the semiconductor. Measurements have shown the interface layer for $\mathrm{Si}-\mathrm{SiO}_{2}$ to be on the order of one micron with surface charge densities of $O\left(10^{10}\right)$ in modern devices. [17, 20,38,56-58]

The last two aspects affecting the surface charge distribution are related by their mobile, cumulative, and Coulombic nature. This makes charge on the outside surface of the passivation and mobile ions in the oxide volume a dominant source of instability.

The mobile ions and similar contaminants may be introduced during the manufacturing process or leech in through an poorly encapsulated oxide-air interface. Once 


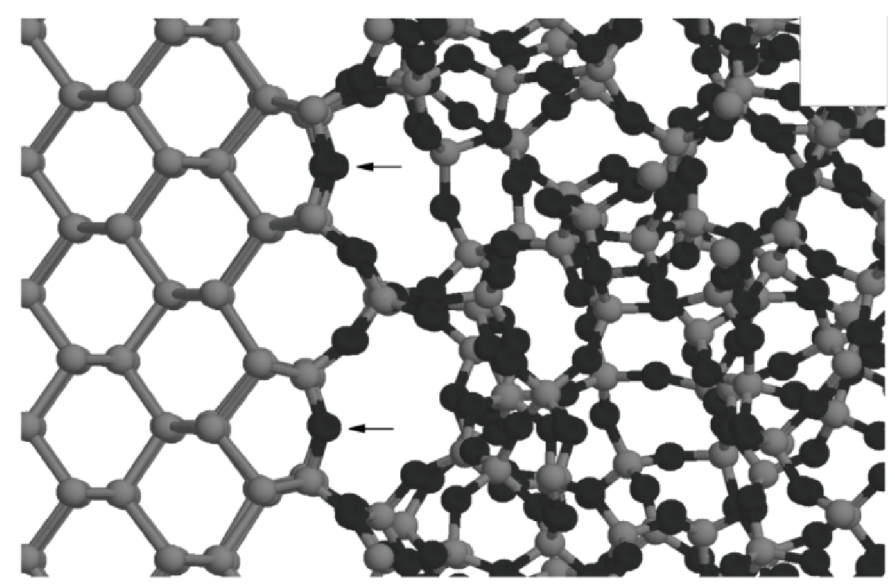

Figure 3.11: An example interface between silicon (left) and silicon dioxide (right). The arrows denote "bridge" bonds which are associated with the large nearby vacancy. [20]

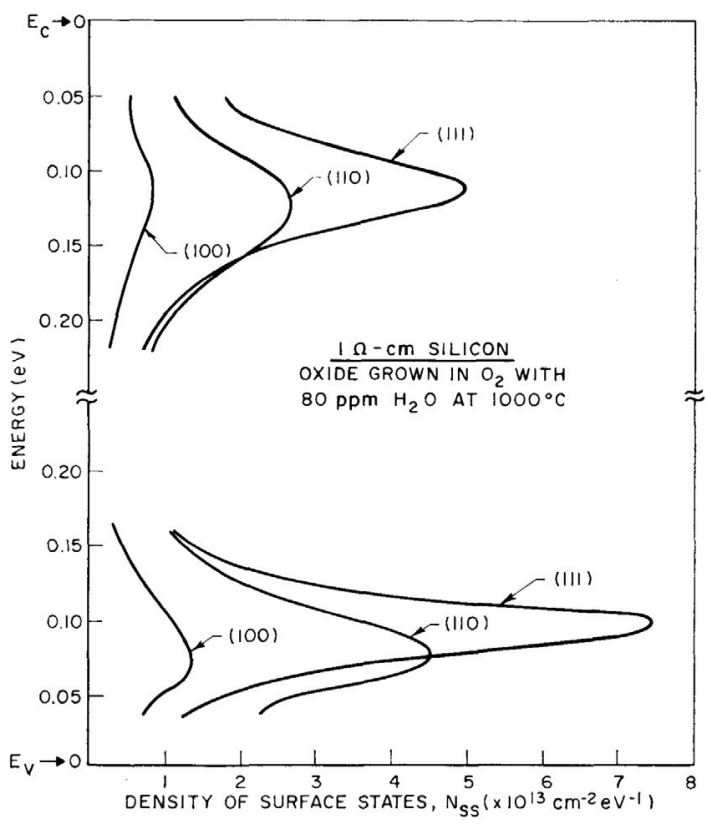

Figure 3.12: Measured surface state densities at the silicon - silicon dioxide interface for three silicon crystal orientations. [17] 
inside they will be propelled by the field as well as attracted by surface charges at the outer and silicon boundaries. The operational bias conditions of the AC-coupled microstrip silicon detector moves positive charges to the silicon interface. The most worrisome being $\mathrm{Na}^{+}$and $\mathrm{H}^{+}$. For $n^{+}-i n-p$ these ions further reinforce the inversion layer. [17, 48, 49, 57, 58]

As discussed the predominant surface effect is a loss of electrical isolation due to the formation of an inversion layer (of minority carriers) that accrues between the implants. These parasitic pathways lead to increased leakage current of the bulk (paths over the edge gap) and the surface (paths between strips). In an ideal detector the inversion layer can be quite small if the dopant level of the semiconductor is chosen correctly. However, in a real device all of the discussed effects will be present and the inversion layer will be altered. In the case of $n^{+}-i n-p$ these effects reinforce the inversion layer and its negative consequences. This necessitates the use of strip isolation structures for this material type to negate the charge sharing and loss of resolution that will occur (ie. must use something like a p-spray or a p-stop).

With current technology such as the FZ methodology excellent bulk material is produced and in most modern unirradiated microstrip devices instability is attributed to the surface. Namely, the high electric field density that can build up in the geometrical system of the strips due to charge accumulation at the silicon interface. This leads to breakdown of the field localized to a small region; microdischarge. As mentioned it is characterized (like microplasma effect) by a short increase in leakage current to a new plateau value. Figure 3.13 shows the IV characteristic of microdischarge. This is contrasted from the junction breakdown which is considered to be sustained current increase [21]. The microdischarge effect has a positive temperature coefficient signifying that it is caused by the avalanche effect. Microdischarge is considered to be reversible and reproducible but can also be the precursor to junction breakdown if left unchecked. Interesting from a diagnostical point of view is that the localized breakdown has been correlated to both localized IR emission and a noise increase in the front-end chip if the sensor is bonded. [17, 21, 22, 29, 49,53]

Both geometry and charge concentration affect the field strength. Figure 3.14 shows an example of a geometrical effect. A test sample with constant implant width and variant aluminum strip width shows that the high field is located at the edge of the implant. Microdischarge onset increases to a saturation point as the metal strip edge is stepped back from the implant. The silicon interface charge determines the 


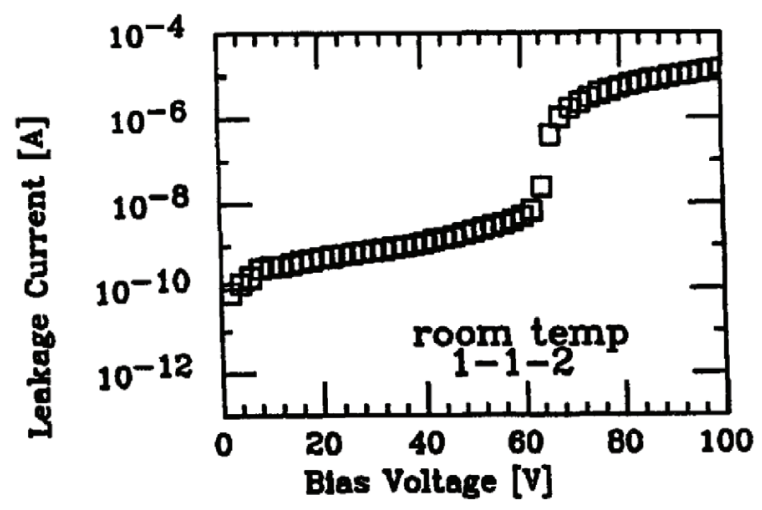

Figure 3.13: IV characteristic of microdischarge. Sensor leakage current as a function of bias voltage and voltage across the integrated capacitor. (Bias from strip to bias implant.) [21]

magnitude at these high field locales. Care must be taken to avoid undue charge from poor manufacturing and poor handling, especially in the more serious case of the $n^{+}-i n-p$ sensor.

The mobile ion effect and the related outer surface charge distribution are considered to be the dominant source of surface instability due to their migratory and cumulative nature. The resistivity at the interfaces (both the silicon interface and outer surface) affects the migration rates of the mobile charges. How quickly they will attain a steady state is dependent upon bias history as well as ambient humidity (since this affects the outer surface resistivity). [17, 21, 22, 53, 56-58]

Aside from increased instability the mobile ions are responsible for observed hysteresis of the IV character $[22,58]$. This is explained by the above resistive nature of the surfaces slowing the mobile ions and moderating the evolution of the field. Both humidity and length of time kept at the preceding bias state contribute to the rate at which the system approaches a steady state. Mobile ion effect is shown experimentally in Figure 3.15 in a $p^{+}-i n-n$ SCT sensor and strong evidence has been seen for its influence in the ITk prototypes (see Section 5.5.3). 

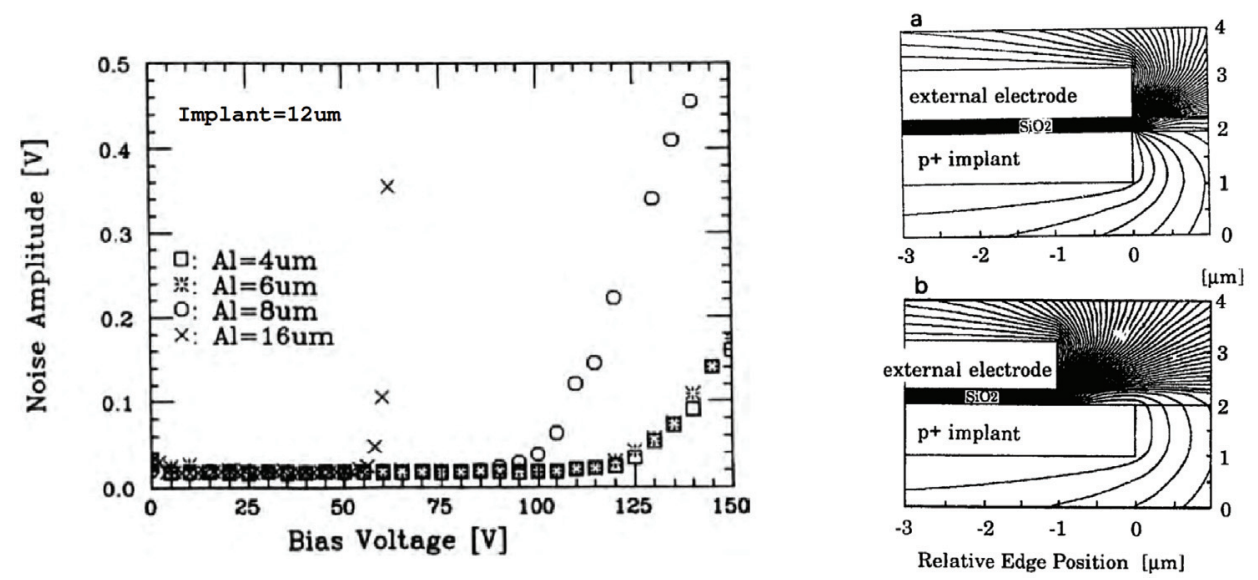

Figure 3.14: An example of the effect geometry plays on field strength at the sensor surface. At left; the onset voltage of microdischarge is noted by its correlated noise increase. Strip widths are varied while the implant width is constant. At right; the explanation for the observed trend. Equipotential lines for the case of congruent edges (a) and with the strip edge stepped back one micron. [21]

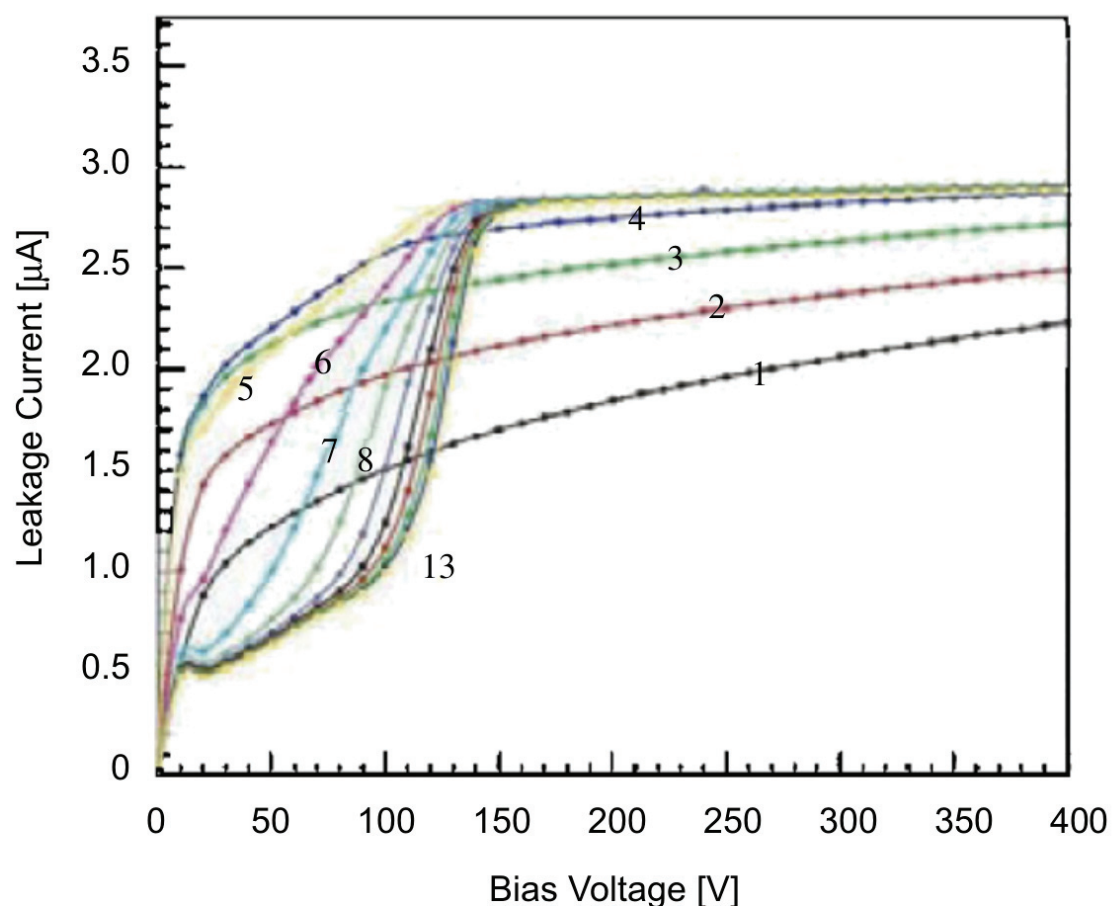

Figure 3.15: Hysteresis in the SCT. Consecutive IV curves taken during long term bias at $150 \mathrm{~V} .4 h r$ intervals in given numerical sequence. [22] 


\subsubsection{Noise}

Since the capacitance of the detector will change with applied voltage (and with irradiation) a charge sensitive pre-amplifier is desired in the front-end chip (and implemented in the ABCStar). Thus noise for the module originates as input capacitance at the front-end. This is stray capacitance that arises in the sensor. The sensor design can protect against the interstrip capacitance driven coupling to neighbouring strips by incorporating considerably higher interstrip resistance than the front-end chip impedance [39]. However, the inductive coupling to external fields (common mode noise) by the metal strip array cannot be mitigated and is the dominant capacitive noise source at the front-end.

Other noise can come from spontaneous fluctuations in the current flowing through, or voltage across the device. It can be traced usually to statistical fluctuations in the parameters of the sensor that affect the charge carrier density. Generation, recombination and trapping rates, diffusion velocities, modulations due to surface charge are all examples. Most of these effects and the contribution from the bulk leakage current itself are smeared out by the integrated capacitor and are overwhelmed by the common mode noise. These considerations allow excellent signal to noise in carefully constructed microstrip detectors since the noise level is negligibly sensor dependent. [17, 24, 39, 48, 59]

\subsection{Radiation Damage}

In the ITk the sensors will see particle fluences and ionizing doses exceeding $1.8 \times 10^{16} n_{e q v} \mathrm{~cm}^{-2}$ and $1.25 \mathrm{MGy}$ (see Table 1.2). This translates to enormous amounts of radiation induced damage in the sensors with implications for operation. The damage can be classified into two categories; bulk and surface. The damage considered in both cases is the introduction of defects into the silicon or oxide due to interaction with the passing particle. As mentioned, for the amorphous $\mathrm{SiO}_{2}$ layer at the surface the concern is with the creation of ionized traps that lead to the nasty accumulation of surface charge. In the bulk a large variety of defects are concerning. These have fundamental consequences for detector operation; altered effective dopant concentration and its increase in the full depletion voltage, increase of leakage current, decrease in charge collection efficiency and its reduction in energy resolution. 


\subsubsection{Bulk Damage}

The two main classes of defect are point (or Frenkel) and cluster. A point defect is the displacement of one (or a few) silicon atoms from the lattice. If recombination does not occur this results in a lattice vacancy and an interstitial silicon. In the case of cluster defects the ejected silicon atom has enough energy to displace more silicon atoms before it comes to a rest. The result is large complexes of vacancies and interstitials along the track of the knocked off silicon that are deep traps for charge carriers. The energy transferred to the silicon in each type of defect formation are different by orders of magnitude [23]. This leads to the association of point defects with Coulombic interactions and cluster defects with collisional interactions. [23, 48, 49, 60, 61]

Naturally the nature of the defects created is closely related to type of radiating particle. Gamma rays are penetrating and drop little energy in the detector and will produce point defects only. The electrically passive neutrons can only interact via collisions to produce cluster defects. While charged hadrons and high energy electrons will behave intermediately, producing both types of defects in concentrations dependent upon their energy. [23, 48, 49, 60, 61]

To understand the effect of the defects upon the detector operation requires a knowledge of their energy level structure. Fortunately, modern methodologies such as deep level transient spectroscopy (DLTS) and thermally stimulated current (TSC) allow a detailed survey of the energy spectrum of the defect levels. As per usual it is the defects with energy levels in the band gap that are of interest. [23, 48, 49, 60,61]

For gamma irradiation two states are considered as important; a deep acceptor level and a shallow donor level. The deep acceptor level is at the middle of the gap and has a quadratic dependence on dose. This signifies that it is a second order effect; interaction of a defect with another defect. It has been attributed to the $\mathrm{V}_{2} \mathrm{O}$ complex (where $\mathrm{V}$ stands for vacancy). Since it is in the middle of the band gap it contributes to leakage current increase. Because it is a deep acceptor it also results in negative space charge buildup in the depleted bulk. The donor defect state has a linear (first order) dependence and contributes a positive space charge. [23, 48, 49, 60,61]

For neutrons, interaction with the lattice is via collisions only. The large energy transfer results in a high energy silicon atom which produces an extended complex of defects in its displaced path. These large defects are correlated with the appearance of deep acceptor levels [23]. Again, the effect is introduction of negative space charge in the bulk. The deep acceptor introduction rate has been measured to be linear with 
neutron dose. Neutrons also produce point defects such as the donor level produced by the gammas but in much fewer number (Coulombic interaction between kicked off silicon by-products and lattice). [23, 48, 49,60,61]

Irradiation with charged hadrons and high energy electrons results in intermediate effects; both Coulombic and collisional interactions can be enacted by the radiating particle. This is similar to the neutron case with the main difference being the rate at which the Coulombic defect is produced. For the charged massive particles the energy deposition via the Coulomb interactions is sufficient to produce higher order point defects (such as the tri-interstitial $\mathrm{E}(30 \mathrm{~K})$ state) that do not occur in nearly as high proportion with neutrons or photons. This can be seen and has important consequences in the annealing curves (Figure 3.22) discussed below. The proportion of point to cluster defects that a charged particle creates is related to its energy. $[23,48,49,60,61]$

The deep acceptor states that arise lead to a build up of negative space charge in the bulk. These are shown to overpower the small increases in positive space charge due to predominantly shallow donors. In p-type silicon the negative space charge increases the effective doping concentration on the bulk side of the junction. This in turn raises the full depletion voltage. In a n-type bulk this negative space charge build up leads to "type inversion", the bulk loses its n character in favor of p. Type inversion is hugely problematic for operation of a microstrip detector causing the junction (collection site) to move to the opposite surface of the detector. It occurs at integrated fluences on the order of $10^{13} \mathrm{~cm}^{-2}$ and would necessitate double sided sensors in the ITk if the $n^{+}-i n-p$ design was not adopted (ie. sensors with microstrips patterned on both faces). The nearly mid-gap deep acceptors also contribute to the leakage current increase. This is shown in Figure 3.16 where the leakage current increase as a function of neutron induced defect concentration (measured directly using DLTS) is plotted. The relative contributions to bulk leakage current from gamma deep acceptor and hadron deep acceptor states can be seen Figure 3.17 for recent ATLAS ITk Strip prototypes at fluences on the order of the HL-LHC expectations (note that surface damage also contributes, and is considered as non-negligible for the gamma case). Recent work has shown that the temperature dependence of leakage current is well modelled by Equation 3.6 up to fluences of $1 \times 10^{15} n_{\text {eqv }} \mathrm{cm}^{-2}$ [52]. [23,24,48,49,60-62] 


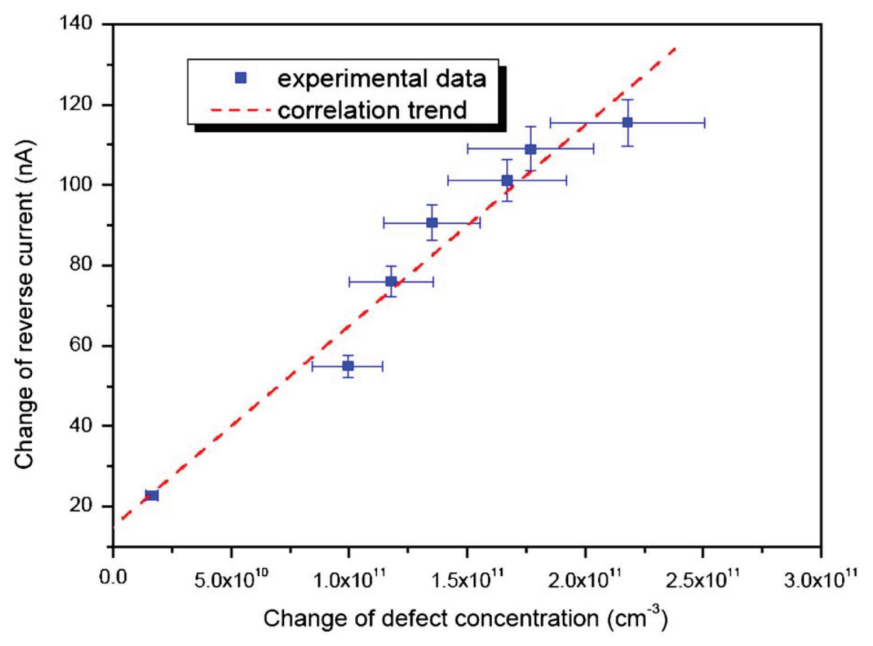

Figure 3.16: Correlation between the neutron radiation induced deep acceptor states and leakage current increase. The deep acceptor state concentrations are measured directly using DLTS. [23]

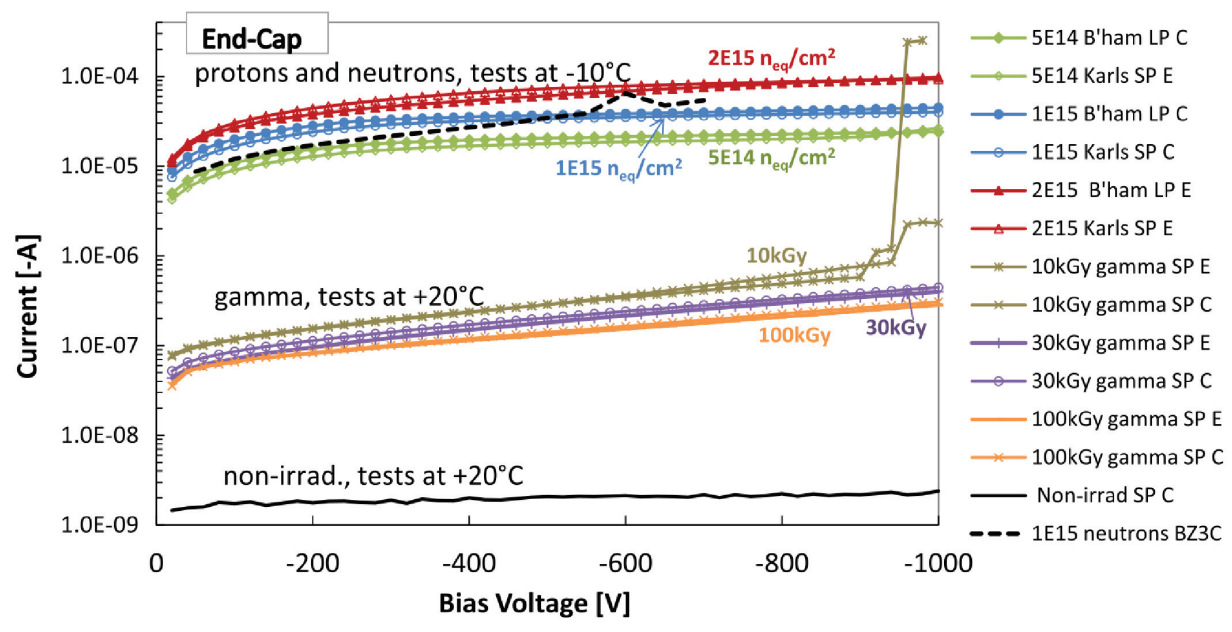

Figure 3.17: Leakage current of recent ATLAS ITk Strip prototype minis (ATLAS12) with and without radiation of different types and fluence. The relative contributions of each type of irradiation to the leakage current increase expected for doses in the HL-LHC. [24] 

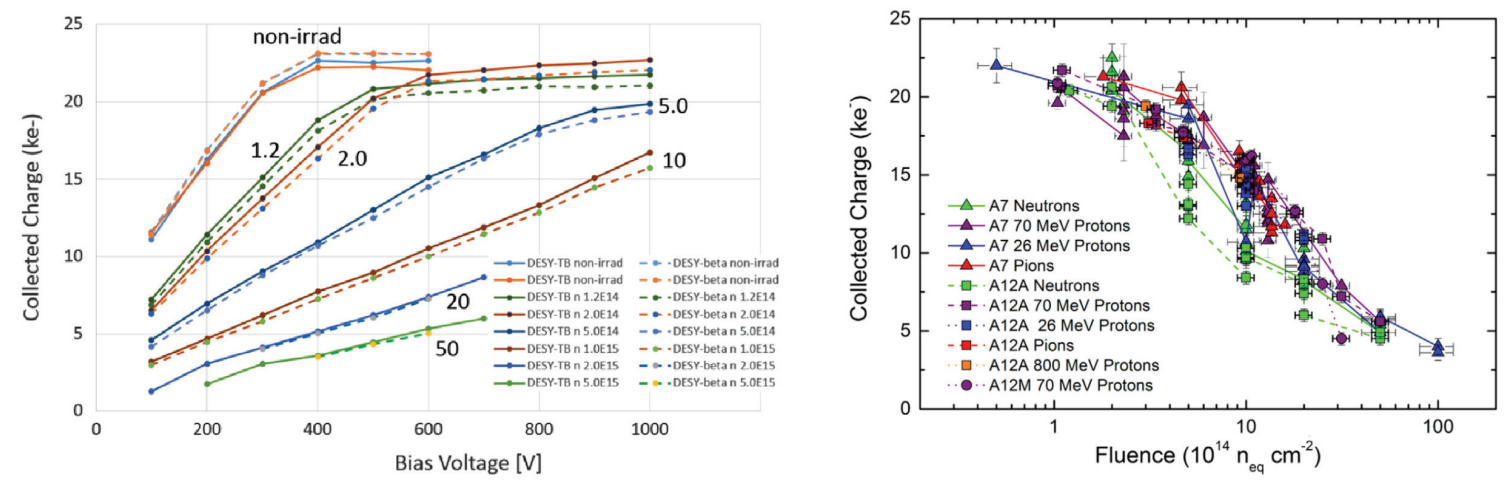

Figure 3.18: Irradiation effect on charge collection efficiency. Left; collected charge as a function of bias voltage for different neutron fluences. The unit for the numbers next to the curves is $\times 10^{14} n_{\text {eqv }} / \mathrm{cm}^{2}$. Results from two sources (testbeam and beta source) are shown. [25] Right; the evolution of collected charge with fluence for neutron and proton irradiated samples. The NIEL hypothesis is shown. [26] Samples are ITk prototypes (ATLAS07 and ATLAS12) and have been annealed $10 \mathrm{~min}$ at $80^{\circ} \mathrm{C}$.

As expected, CCE is also affected by radiation; degradation due to the introduction of trapping sites occurs. Figure 3.18, left, shows this effect with recent ATLAS ITk Strip prototypes irradiated with reactor neutrons. Due to the interrelation of charge collection and depletion depth this plot also shows the increase in full depletion voltage. As discussed in Section 1.4.3 the radiation damage (specifically the deep acceptor states) is roughly proportional to non-ionizing energy loss (NIEL). This allows different radiation types to be compared by use of proportionality constants and plots like Figure 3.18, right, to be produced. The collected charge versus bias voltage for variously irradiated samples is shown.

The probability for a trap to capture a carrier is related to the carrier's drift velocity. In this way, application of higher voltages can reduce the CCE losses, this is however limited by sensor breakdown and power consumption considerations. [23, $25,48,49,60-62]$

\subsubsection{Surface Damage}

At the surface it is ionizing damage which matters. Charged particles and high energy photons will ionize atoms in the amorphous silicon dioxide layer leading to the introduction of trapping states. The ionization leads to positive space charge 
build-up as the field drifts the electron out of the oxide. In p-type bulk devices this will reinforce the MOS structure's inversion layer due to the accumulation of mirror charges in the silicon. This in turn contributes to the parasitic pathways that lead to increased leakage current between the junction poles and between the strip implants. The negative connotations of increased bulk leakage current have already been discussed. In the interstrip region the increased conductive layer of electrons between the two implants lowers the interstrip resistance. Implementation of strip isolation structures can only reduce this effect. Interstrip resistance measurements of recent ATLAS ITk Strip prototypes as a function of ionizing fluence are shown in Figure 3.19. The interstrip capacitance is also somewhat affected by the surface radiation damage. At voltages less than full depletion, the build up of the mirror negative charge in the volume increases the capacitance. As bias is increased towards full depletion these charges are confined to a smaller layer at the interface and the interstrip capacitance approaches its non-irradiated value. This effect is seen in Figure 3.20 for proton and gamma irradiation. Although this is seemingly a first order effect it has been shown to have a dose ${ }^{2 / 3}$ dependence [53]. [24, 25, 53, 56]

\subsubsection{Annealing}

Annealing is the rearrangement of the lattice (or lattice charge) by thermal means. It occurs naturally at any non-zero temperature in a statistical manner over time and thus can be accelerated if the thermal energy is increased. While it is not a radiation damage effect it is associated with them since one of the effects of irradiation is to introduce lattice defects in the bulk. The lattice rearrangement can change the nature of the defects activating electrically inactive sites and vice versa. Thus annealing can be both beneficial and detrimental to the sensor operation by either decreasing or increasing the effective defect concentrations.

Since the base material is silicon, the same classes of defects are produced with relatively similar concentration proportions across different samples. This leads to characteristic annealing curves as shown in Figure 3.22. Reactor neutron irradiated samples are shown on the left and $23 \mathrm{GeV}$ proton irradiated samples on the right. Space charge concentrations are measured as a function of annealing time (coloured trends) allowing the densities of acceptor and donor states to be extracted. Here, 

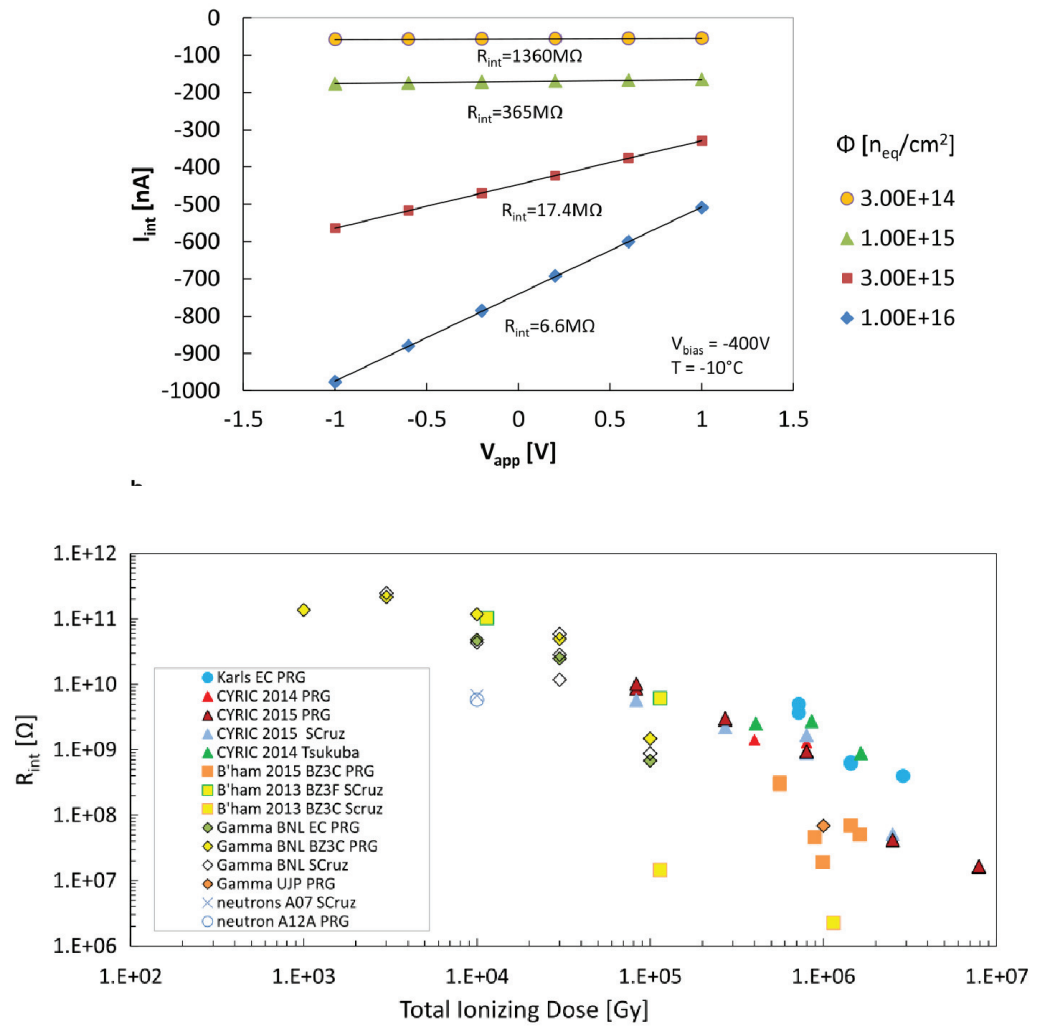

Figure 3.19: The effect of surface damage on interstrip resistance. Results are for recent ATLAS ITk Strip prototypes (ATLAS12). Top, direct measurement of interstrip resistance for different proton fluences. The methodology outlined in Section 5.1.1 is used. Bottom, the interstrip resistance as a function of the total ionizing dose for samples irradiated with protons, neutrons, and gammas. [24] 

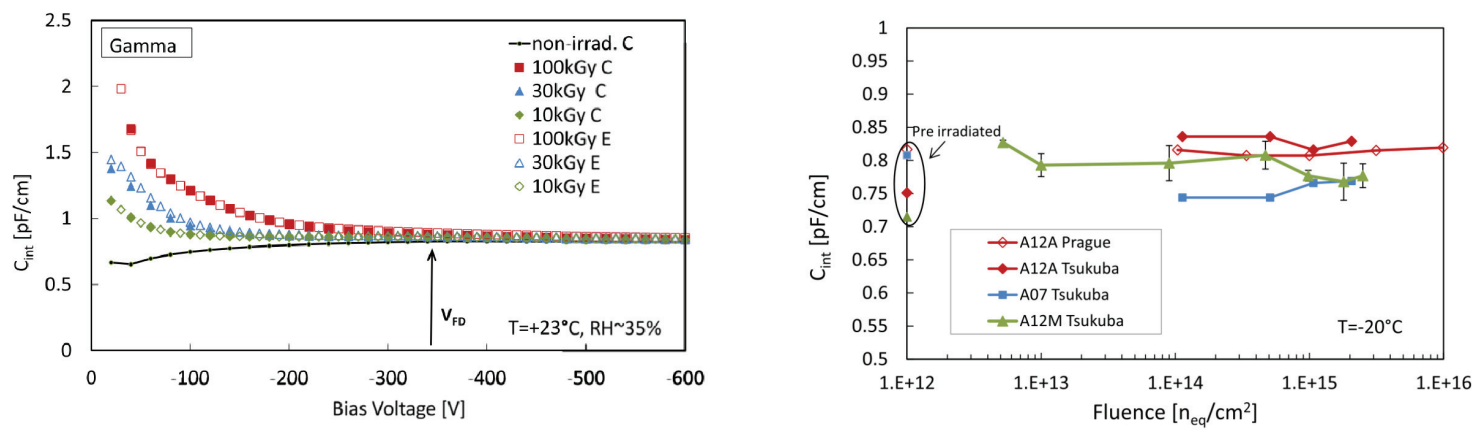

Figure 3.20: The effect or surface damage on interstrip capacitance. Results are from recent ATLAS ITk Strip prototypes (ATLAS12 and ATLAS07). At left, the interstrip capacitance as a function of the bias voltage after various levels of gamma irradiation. Confinement of the induced mirror charge to the thin inversion layer at higher voltages can be seen. At right, interstrip capacitance at very high voltage $(-1 \mathrm{kV})$ as a function of proton fluence on various samples. Saturation to the roughly un-irradiated value is evident. [24]

"E" is the name given to the common $V-P$ defect which removes p-type dopant (phosphorous). The most important curve is that of the CV inferred effective doping concentration shown in black. In both cases a minimum in the defect concentration occurs at roughly ten minutes of annealing at 80 centigrade; this defines the (conventional) beneficial annealing regime. If annealing is continued then the defect concentration increases; this detrimental effect is termed reverse annealing.

Beneficial annealing has been attributed to the higher order point defects (such as $\mathrm{E}(30 \mathrm{~K})$ ) which coalesce from electrically inactive defects after a minor annealing. This can be seen most markedly by comparing the green and blue curves in Figure 3.22 for neutron (no $\mathrm{E}(30 \mathrm{~K})$ defect) and proton $(\mathrm{E}(30 \mathrm{~K})$ formation) irradiation.

For reverse annealing the cluster related deep acceptor states which contribute negative space charge are considered as culprit. The existence of reverse annealing purporting the existence of defects that only become electrically active after sufficient rearrangement. For both cases the quadratic appearance of the dip and rise lend credence to a second order effect.

The effect of a beneficial annealing period of ten minutes at $80^{\circ} \mathrm{C}$ is displayed clearly in Figure 3.21 for both neutron (left) and proton (right) irradiation. The annealing of the IV curve is seen on the left in Figure 3.23 for a $23 \mathrm{MeV}$ proton irradiated sample. At low voltage the deep acceptor state damage reduces the depletion depth and at higher voltage increase the field strength until avalanche occurs. 

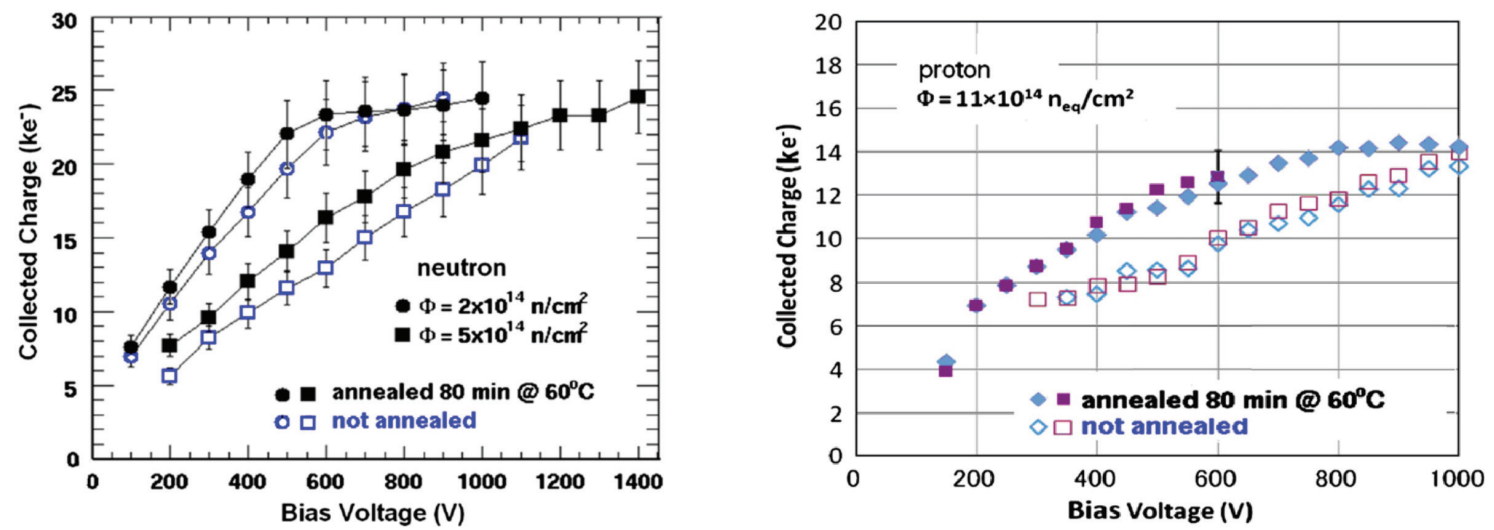

Figure 3.21: Clear advantage of beneficial annealing of $10 \mathrm{~min}$ at $80^{\circ} \mathrm{C}$. Charge collection curves of ITk prototypes (ATLAS07) with and without annealing. Left; reactor neutron irradiation at two fluences. Right; $70 \mathrm{MeV}$ proton irradiation to the fluence of $1.12 \times 10^{15} n_{e q v} \mathrm{~cm}^{-2}$. [26]

The effect of reverse annealing of the interstrip resistance is shown in Figure 3.23, right. With higher deep acceptor defect concentrations the interstrip resistance is seen to increase at low voltage due to the disturbance of the inversion layer formation. At high voltage the interstrip decreases as high fields cause charge multiplication. [23, 24, 48, 49, 56, 60, 61, 63] 

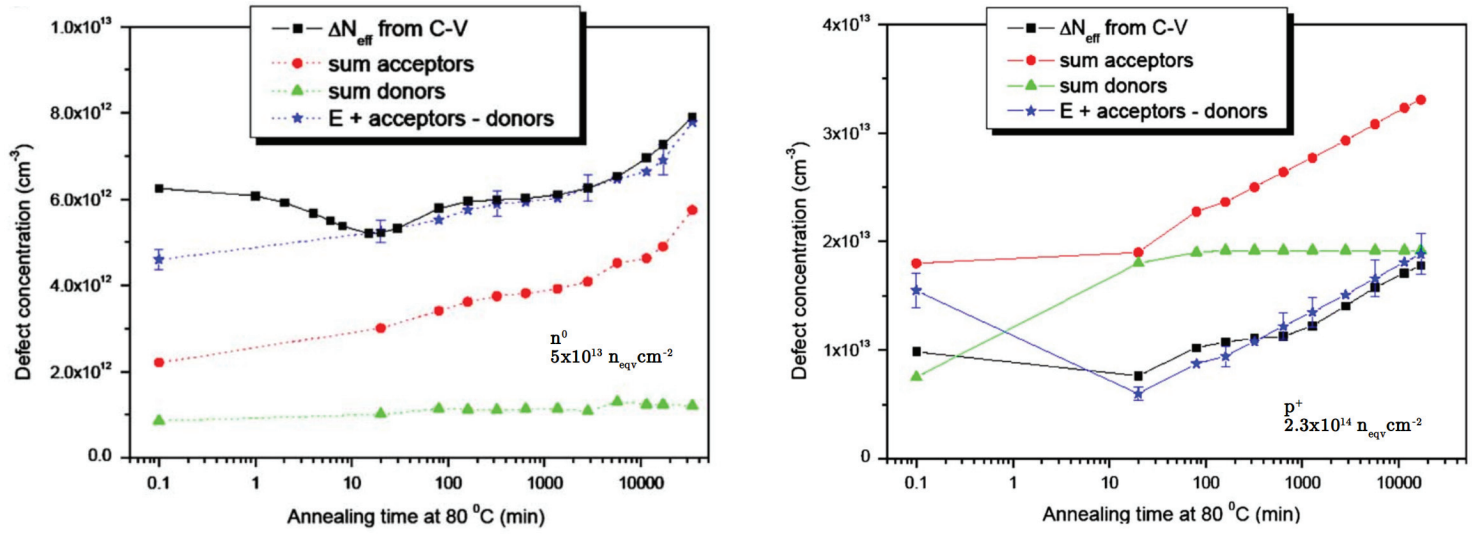

Figure 3.22: Annealing of the irradiation induced space charge concentrations and $\mathrm{CV}$ inferred effective doping concentration. E refers to the $V-P$ defect which removes shallow donor levels. At left, neutron irradiation to a fluence of $5 \times$ $10^{13} n_{\text {eqv }} / \mathrm{cm}^{-2}$. At right, proton irradiation to $2.3 \times 10^{14} n_{\text {eqv }} / \mathrm{cm}^{-2}$. Measured and model predictions are shown, results are for oxygenated float zone substrate. [23]
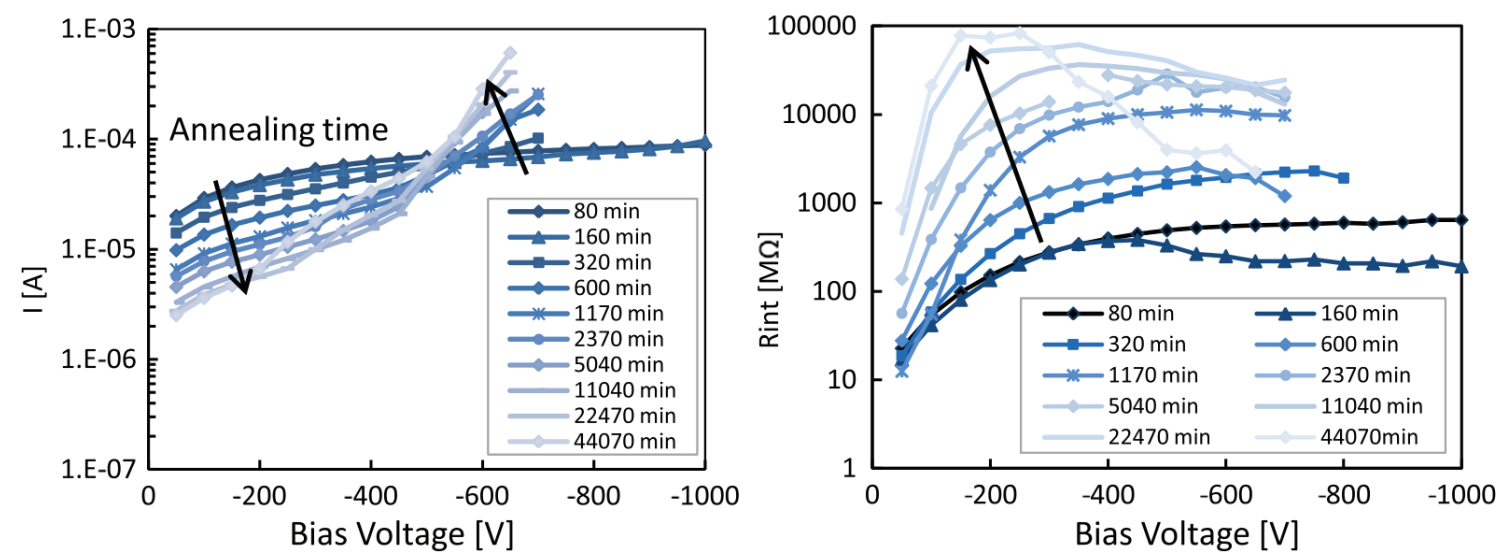

Figure 3.23: Effect of consecutive annealing periods at $60^{\circ} \mathrm{C}$ of a $23 \mathrm{GeV}$ proton irradiated ATLAS ITk Strip prototype (ATLAS12) to a fluence of $2 \times 10^{15} n_{\text {eqv }} / \mathrm{cm}^{-2}$ . At left, leakage current. At right, interstrip resistance. [24] 


\section{Chapter 4}

\section{Sensors of the ITk Strip Detector}

The underlying physics behind microstrip detectors described in the preceding chapter offers the contextual basis for a detailed discussion of the novel sensor designed for the ITk Strip Detector. The main topic of this thesis is the implementation of a prototype and production test site at Carleton University, as discussed in Chapter 5. For its successful implementation a full understanding of the unique features and rich history of the sensor must be known. In this manner subtleties arising in the unique design which includes several significant advancements in the microstrip field can be addressed and a meaningful impact can be made.

In this chapter the ITk sensor design is highlighted. A brief introduction into the features, specifications, and geometries of the sensor design is given in Section 4.1 and allows the transition to an investigation of the Strip sensor's pioneering work. The approach for treatment of this matter is first a chronological presentation of the evolution of the ITk Strip design, given in Section 4.2. This gives experimental context for a further look at the integral advances in the understanding and design of microstrip detectors that resulted from the project, as given in Section 4.3. It can be noted that the outline of the sensors presented in Section 4.1 is for the planned ITk sensors; the specifications do not exactly match those for the prototype modules discussed in the remainder of this and the following chapter.

\subsection{Overview and Specifications}

In the ITk two challenges for a solid state detector are taken to unrivalled extremes; surface coverage and radiation hardness. The technology to cope with these requirements is not trivial to design or implement. The sensors of the ITk Strip detector 
utilize a number of unique features that have been cultivated specifically for the ITk and HL-LHC environment.

Table 4.1 summarizes the specifications for the ATLAS ITk Strip microstrip silicon sensor. Over the course of this chapter (and the last) the majority of these parameters will be addressed.

\subsubsection{Introduction to the ITk Strip Sensor Design}

The ITk Strip sensor is a large area, $n^{+}-i n-p$, AC-coupled microstrip, oxygenated float-zone (FZ) silicon detector. Central to the radiation hardness and cost effectiveness of the design is the uncommon $n^{+}-i n-p$ material choice. A modern advancement in the silicon microstrip detector field initiated in the LHC community, this attribute nullifies the degrading effects of type inversion. Specifically, this allows a single sided process, and operation in partial depletion. Furthermore, the collection of electrons promises a faster and potentially larger signal [29]. The one disadvantage of the $n^{+}-i n-p$ design (that of strip isolation) is overcome with the implantation of a novel $p^{+}$trace structure between the strips, the so called "p-stops", which have been optimized for radiation hardness.

An AC-coupled readout protects the front-end chips from the bulk leakage current which offers several advantages in terms of signal to noise ratio in the assembled module (see Section 3.3.7). Since the bulk leakage current increases with irradiation this feature is crucial for the end-of-lifetime performance [21,64]. A conventional $\mathrm{SiO}_{2}$ dielectric layer is used to couple the $n^{+}$implants and chemical vapour deposited (CVD) aluminum strips. It covers the full surface, sitting atop the p-stops as well. If carefully designed, the $\mathrm{AC}$ coupling can also protect the front-end from damaging voltage pulses which can be on the order of half the bias value [39] if there is large charge liberation in the bulk (in the event of accidental beam splash). In the ITk Strip sensor the dielectric thickness is tuned to give a breakdown voltage above $100 \mathrm{~V}$. To protect breakdown of the dielectric a newly developed punch through protection (PTP) structure with turn-on voltage of $20-25 \mathrm{~V}$ is implemented. It takes form as a small gap between the implant and a poly-silicon bias rail extension in a novel orientation optimized for high radiation environments.

Reverse bias is distributed to the strips via an array of poly-silicon bias resistors that mate the ends of the implants to the common $n^{+}$implant of the bias rail. The 


\begin{tabular}{|c|c|}
\hline \multicolumn{2}{|l|}{ Substrate Material } \\
\hline Size & 8-inch/200 mm or 6-inch/150 mm \\
\hline Type & p-type FZ \\
\hline Crystal orientation & $<100>$ \\
\hline Thickness (physical) & $300-320 \mu \mathrm{m}$ \\
\hline Thickness (active) & $>=90 \%$ of physical thickness \\
\hline Thickness tolerance & $\pm 5 \%$ \\
\hline Resistivity & $>3 \mathrm{k} \Omega \mathrm{cm}$ \\
\hline Oxygen concentration & $1 \times 10^{16}$ to $7 \times 10^{17} \mathrm{~cm}^{-3}$ \\
\hline \multicolumn{2}{|l|}{ Sensor specifications before irradiation } \\
\hline Full depletion voltage & $<330 \mathrm{~V}$ (preference for $<150 \mathrm{~V}$ ) \\
\hline Maximum operating voltage & $700 \mathrm{~V}$ \\
\hline Poly-silicon bias resistors & $1-2 \mathrm{M} \Omega$ \\
\hline Inter-strip resistance & $>10 \times$ Rbias at $300 \mathrm{~V}$ at $23^{\circ} \mathrm{C}$ \\
\hline Inter-strip capacitance & $\begin{array}{l}<1 \mathrm{pF} / \mathrm{cm} \text { at } 300 \mathrm{~V} \text {, measured } \\
\text { at } 100 \mathrm{kHz}\end{array}$ \\
\hline Coupling capacitance & $>20 \mathrm{pF} / \mathrm{cm}$ at $1 \mathrm{kHz}$ \\
\hline Resistance of read-out Al strips & $<15 \Omega / \mathrm{cm}$ \\
\hline Resistance of n-implant strip & $<20 \mathrm{k} \Omega / \mathrm{cm}$ \\
\hline Onset of micro-discharge at & $>700 \mathrm{~V}$ \\
\hline Total initial leakage current, including guard ring: & $\begin{array}{l}<0.1 \mu \mathrm{A} / \mathrm{cm}^{2} \text { at } 700 \mathrm{~V} \text { at room } \\
\text { temperature }\end{array}$ \\
\hline Number of strip defects & $<1 \%$ per strip and \\
\hline & $<1 \%$ per sensor \\
\hline \multicolumn{2}{|l|}{ After irradiation $\left(1.2 \times 10^{15} \mathrm{n}_{\mathrm{eq}} / \mathrm{cm}^{2}-50 \mathrm{MRad}\right)$} \\
\hline Onset of micro-discharge at & $\begin{array}{l}>700 \mathrm{~V} \text { or } \mathrm{V}_{\text {fulldepletion }}+50 \mathrm{~V} \\
\text { after irradiation (if lower) }\end{array}$ \\
\hline Inter-strip resistance: & $\begin{array}{l}>10 \times \mathrm{R}_{\text {bias }} \text { at } 400 \mathrm{~V} \text { and for } \\
\mathrm{T}=-20^{\circ} \mathrm{C}\end{array}$ \\
\hline Collected charge & $>7500$ electrons per MIP at $500 \mathrm{~V}$ \\
\hline \multicolumn{2}{|l|}{ Mechanical Specifications } \\
\hline Dicing precision & $< \pm 20 \mu \mathrm{m}$ or better \\
\hline Sensor bow after process and dicing & $<200 \mu \mathrm{m}$ \\
\hline
\end{tabular}

Table 4.1: A summary of the specifications for ITk Strip detector's large area, $n^{+}-i n-p$, AC-coupled microstrip, oxygenated float zone (FZ) silicon detector. [1] 
poly-silicon is implanted in a meandering path utilizing the full pitch availability. A design that maximizes the strip length and bias resistance simultaneously. Since the implants are AC-coupled in the manner outlined, the bias rail provides the return (ground) connection. The reverse bias' high voltage source connection is made to the $p^{+}$, Al-metallized backplane of the sensor. For stability considerations the cut edges of the sensor are processed with $\mathrm{SiO}_{2}$ with the result of high conductivity (acts as a self conductive FOXFET). Thus the edges are at the same high voltage as the backplane. A front-side edge metal structure anchors a guarding $p^{+}$edge implant which corrals the field as well as offers a top surface connection to the backplane. A single floating $n^{+}$guard ring is used in an unusual slim edge design $(450-500 \mu \mathrm{m})$ which minimizes dead area in the detector.

For power consumption, the reverse leakage current across the enormous silicon surface must be minimal. Ultra-pure, mono-crystalline p-type silicon is grown via the float-zone method (using the surface tension of molten silicon to carry away contaminants from a solidifying core). Radiation hardness (in the form of defect control) is offered by purposeful introduction of higher interstitial oxygen concentrations, a recent advance pioneered for the HL-LHC [33, 35, 65].

Since the sensor surface is so large an essential consideration for stable long-term operation is surface passivation. A combination of $\mathrm{SiO}_{2}$ and $\mathrm{AlN}$ : $\mathrm{H}$ (hydrogenated aluminum nitride) is used to cover the top and edge surfaces [50]. This mixture is a conventional and effective passivation material for silicon detectors [66] . A number of different windows in the passivation exist to facilitate top surface electrical contact. Each of the edge structures (edge-metal, guard ring and bias ring) have several windows. Only the bias rail connection is used during operation (for common strip ground) while the others are necessary for full characterization of the sensor. On the strips the passivation windows correspond to the FE chip bonding pads. These socalled AC pads are realized as short widenings which offer ample space for a reliable bond. The final set of passivation windows correspond to an array of small pads connected directly to the implants (the DC pads).

The $300-320 \mu \mathrm{m}$ thickness of the ITk Strip sensor is similar to that used in the SCT $\left(285 \mu \mathrm{m}\right.$ [64]). Since the $n^{+}-i n-p$ design allows partial depletion operation this choice is a compromise mostly between signal size and radiation length. A thicker sensor offering more volume from which to liberate charge but also increasing the radiation length of the sensor (which is already the largest contributor at the module 
level, see Figure 1.9).

In Figure 4.1 the surface of the current iteration of ATLAS ITk Strip sensor (ATLAS12EC) is shown at two magnifications. The majority of aforementioned features can be discerned. Focusing on the lower magnification photo first; the three edge structures can be easily found as the outermost lighter objects. This lighter colour signifies the CVD aluminum (usually anchoring an implant). Moving inwards from the edge, one encounters the edge-metal, guard ring, and bias ring. The edge-metal and bias rail are denoted with fiducial marks which assist in the automation of the assembly process. Strip numbers are also found along the edge metal perpendicular to the strips. The large array of thin aluminum strips with the staggered widenings of the AC pads are easily identified at low magnification. The presence of the dense trace of the poly-silicon bias resistor is likewise obvious at the end of the strip (connecting it to the bias rail). The observable markings on the $\mathrm{AC}$ and bias rail pads are from strip characterization probe tests as described in Section 5.1. A hint of the passivation layer is seen in the greenish tinge along the sensor edge.

At higher magnification the focus is on the end of strip region, the detail and contrast is remarkable and reveals the specifics of the structure. The termination of the lighter coloured aluminum strip is seen to reveal the dark orange of the $n^{+}$ implant. Its path is briefly obscured by another aluminum deposit, that of the DC pad which is directly coupled to the implant. The dense dark bias resistor region resolves to the greenish appearing weave of the poly-silicon bias resistor overlaying the dark orange implant which extends almost to the bias rail. These two features are implanted at different depths and insulated, the electrical mating is visible at the DC pad. The terminus of the $n^{+}$implant is avoided by the meandering poly-silicon bias trace and is seen to be overlain by a light appearing poly-silicon extension from the bias rail. Again the features are at different depths, this time making the fully gated structure of the punch through protection.

Also visible under high magnification are the p-stop's $p^{+}$traces which sits under the $\mathrm{SiO}_{2}$ layer and isolate the strip implants. These appear as faint green lines which sit between the strips and encompass the bias resistor and PTP structure. While the p-stops are seen to be shaped to accommodate the bond pad regions they sit straight and equidistant between the strips in the majority of the surface. Focusing on the edges of the rectangular pads a careful eye can also pick out the slight colour variation that signifies a window in the passivation layer. 

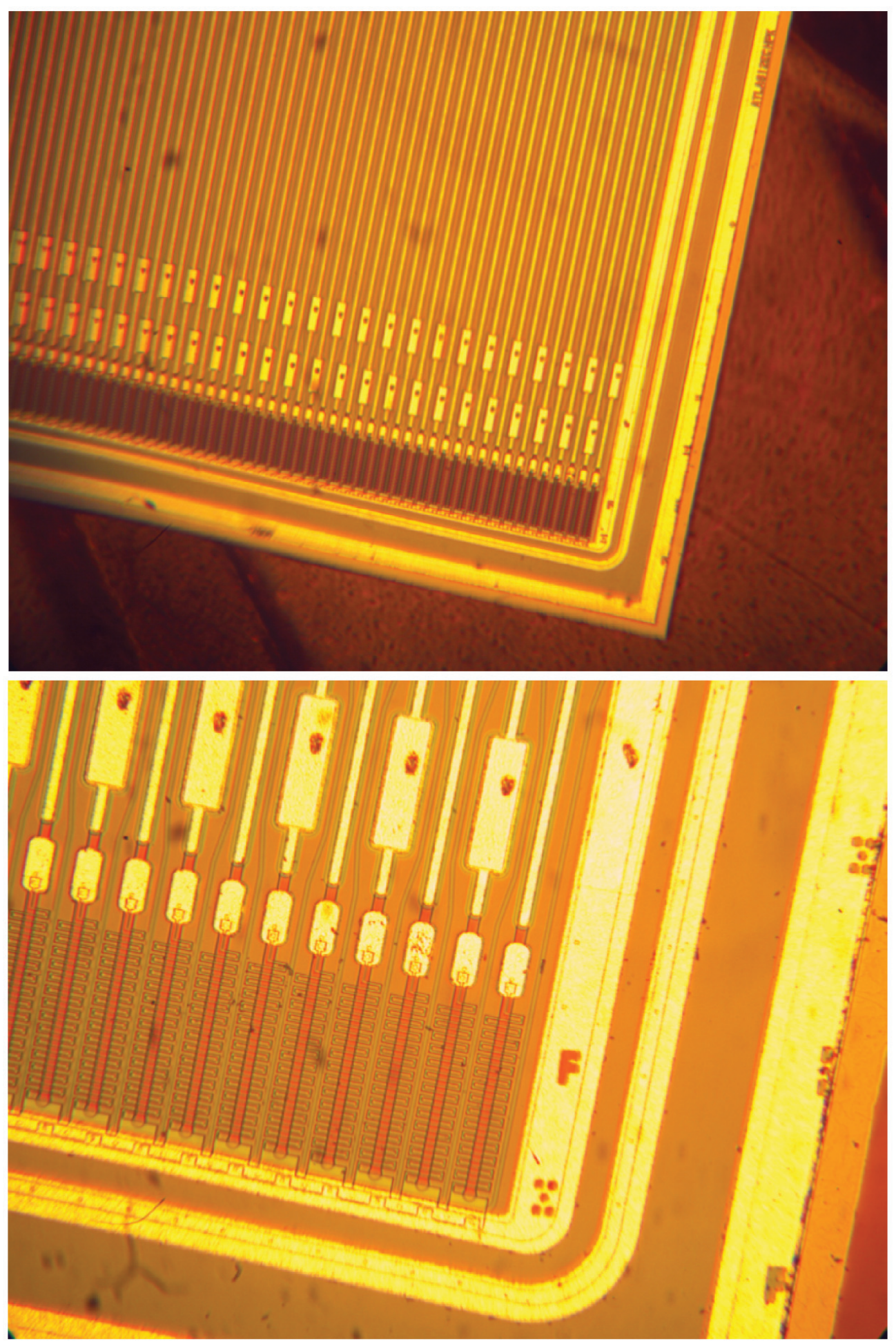

Figure 4.1: A photograph of the surface of the ATLAS ITk Strip sensor prototype taken at Carleton University. Most features of the sensor can be seen, refer to the text for the details. 


\subsubsection{Sensor Geometries}

The ITk will use eight sensor geometries optimized for occupancy, coverage, and readout latency in the dense, fast-paced environment of the HL-LHC. The main parameters for each sensor type are summarized in Figure 4.2. The strip geometries were detailed in a tracking capacity in the similar tables of Section 2.3 (Tables 2.2 and 2.3).

The two barrel sensors are extremely similar having the same physical size and shape. They benefit from the simple geometry of the stave structure and utilize parallel strips in a square sensor with side length a couple of millimetres less than $10 \mathrm{~cm}[1,31,67]$. This sensor size is chosen to maximize the usable area of a six inch wafer (see Figure 4.4). With the widths of the edge structures dictated by stable operation an active area is defined. The strip pitch is then chosen from the tracking requirements and what is reasonable to fit an integer number of front-end chips on the sensor. The value of $75.5 \mu \mathrm{m}$ is used throughout the barrel, this accommodates 1282 parallel strips in the active width. 1280 of the strips are bonded to the input of the 10 ABCStar sitting on the barrel hybrid(s). The two outermost strips are not readout and serve to shape the electric field for their neighbours, minimizing edge effects.

The difference between the two barrel sensors lies in the length of the strip and implant structures and thus number of rows of strips incorporated in the $10 \mathrm{~cm}$ long sensor. It is the lengths that are determined by the strict granularity requirements of the HL-LHC as well as by latency considerations. Four rows of $24.1 \mathrm{~mm}$ long strips are used in the two innermost barrel layers. In the two outer layers the strip length can be doubled and only two rows of strips are used. Table 2.2 showed the details of this configuration in the larger barrel context. In both cases the rows are segmented by a bias rail crossing the sensor width (thus sensor segment is used synonymously with strip row). The end of strip structure detailed in Figure 4.1 is repeated at both ends of the strip, allowing one set of pads to be reserved for the bonding of the FE.

In the endcap everything is made much more difficult by the fan shape of the petals. As seen (Section 2.2) each petal consists of nine fan-shaped silicon sensors in six geometries. The three inner rings with one sensor each and the three outer rings with two identical sensors each. The shapes and sizes are chosen to fulfill the geometrical coverage and tracking requirements while minimizing the number of six 


\begin{tabular}{|c|c|c|c|c|c|}
\hline $\begin{array}{l}\text { Sensor } \\
\text { type }\end{array}$ & $\begin{array}{c}\text { Number } \\
\text { of sensors }\end{array}$ & Shape & $\begin{array}{l}\text { Number } \\
\text { of rows }\end{array}$ & $\begin{array}{l}\text { Channels } \\
\text { per sensor }\end{array}$ & $\begin{array}{c}\text { Min/max pitch } \\
(\mu \mathrm{m})\end{array}$ \\
\hline Short-strips & 3808 & Square & 4 & 5128 & 75.5 \\
\hline Long-strips & 7168 & Square & 2 & 2564 & 75.5 \\
\hline EC Ring 0 & 768 & & 4 & 4360 & $73.5 / 84$ \\
\hline EC Ring 1 & 768 & & 4 & 5640 & $69 / 81$ \\
\hline EC Ring 2 & 768 & & 2 & 3076 & $73.5 / 84$ \\
\hline EC Ring 3 & 1536 & $\ldots$ & 4 & 3592 & $70.6 / 83.5$ \\
\hline EC Ring 4 & 1536 & . & 2 & 2052 & $73.4 / 83.9$ \\
\hline EC Ring 5 & 1536 & 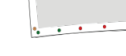 & 2 & 2308 & $74.8 / 83.6$ \\
\hline
\end{tabular}

Table 4.2: An overview of the eight ITk Strip sensor geometries and their main characteristics. The minimum and maximum pitch is across all strip rows. [1] 

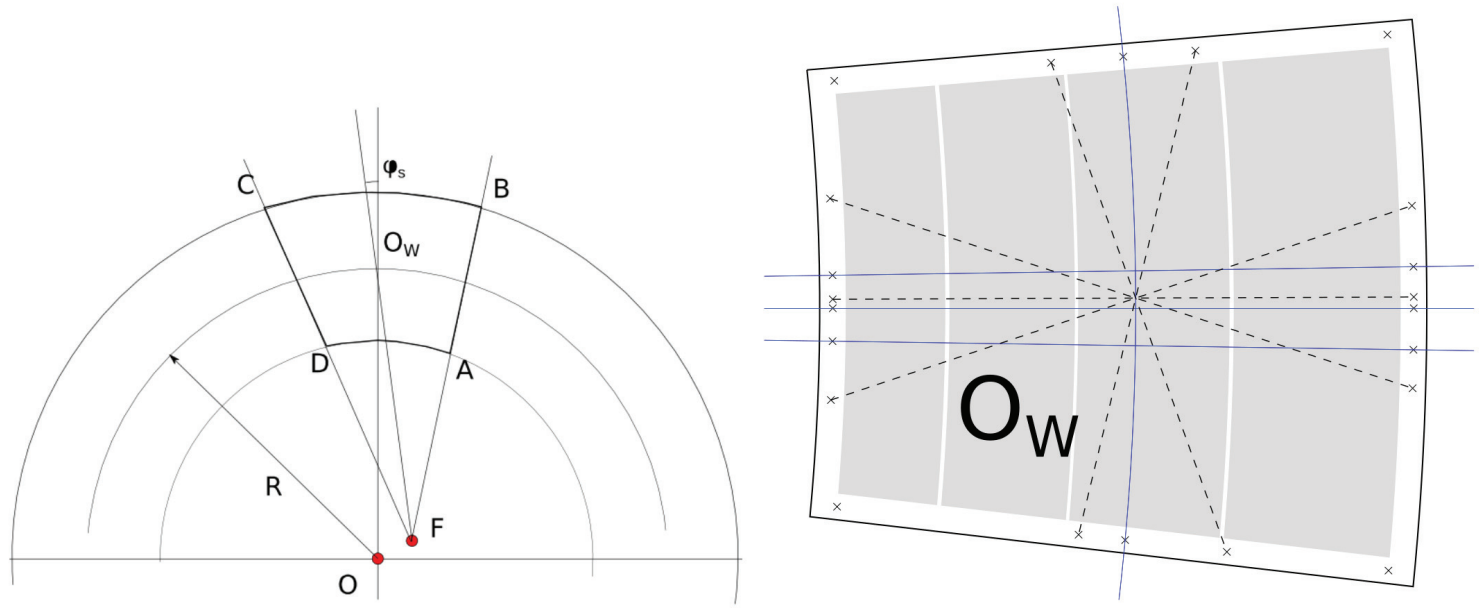

Figure 4.2: The ITk Strip endcap's "stereo annulus" geometry. At left; an exaggeration of the geometry depicting the principle. At right; the stereo annulus geometry as applied to the prototype of the innermost sensor, the ATLAS12EC. [1]

inch silicon wafers required.

In the endcap, for 'simplicity' the stereo angle for the second coordinate measurement (this time radial distance from beam axis) must be implemented directly into the sensors. This gives the endcap sensors the characteristic stereo annulus geometry as seen in Figure 4.2 and discussed in more detail in Section 4.3.3. The strips and straight edges of the sensor do not point to the beam axis but rather are rotated by the $20 \mathrm{mrad}$ stereo angle. The circular edges of the sensor constituting the annulus portion are beam axis centred which minimizes the dead space between rings and allows equal length strip segments [1,30]. In practice the circular edges are approximated during manufacturing using 16 flat cuts of equal length per ring edge per petal [30].

In the barrel, strips are parallel and strip pitch is constant. In the endcap, sensors have the stereo annulus geometry which allows the strips to have a constant angular pitch. Again the pitch is chosen from the active width and the ability to provide strips to an integer multiple number of ABCStar ASICs (cost effectively using the exact same FE chip as the barrel). Thus the pitch stays in the range of 69 to 84 micron while, naturally, the number of strips and ABCstar increases (from seven to 18) moving outward in radius. The strip pitches are detailed for each sensor segment in Tables 4.2 and 2.3. 
As in the barrel the strip lengths are determined by occupancy considerations; shorter strips are found closer to the beam axis and longer ones further away. However, the geometry factors of ABCStar bonding and petal assembly affect the particular lengths of each strip row and dictate the number of segments per sensor. Table 2.3 showed the details for each sensor segment.

\subsection{Development of the ITk Strip Sensor Design}

During the HL-LHC phase the environment at the ATLAS interaction point will be otherworldy (even when compared to a world bearing the LHC). The level of radiation will be too high for any gas detectors and the move to a fully solid state inner tracker is required. The result is enoromous silicon surface coverage in the ITk Strip detector which extends from a radius of about $30 \mathrm{~cm}$ unto the full extent of the inner ATLAS cavity. The approximately $165 \mathrm{~m}^{2}$ is a factor of 2.6 greater than seen in the entire ID.

The increase in integrated luminosity at the HL-LHC, by a factor of 10, results in an increase in the $1 \mathrm{MeV} n_{\text {eqv }}$ particle fluence by an order of magnitude from the LHC's SCT expectation $\left(\sim 2 \times 10^{14}\right.$ to $\left.\sim 2 \times 10^{15}[68]\right)$. This is coupled with a factor of five or more increase in instantaneous luminosity. The unique challenges in this environment necessitate the development of a novel sensor design. New radiation hard materials with optimized dopant types, dopant concentrations, growth procedure, crystal orientation, and operating guidelines have been developed. These have been mated with well refined layout considerations implementing newly developed strip isolation and punch through protection structures, slim edge designs, and stereo annulus geometries.

Engineering, even at this level, can only bring the sensors so far, extending the effective radiation hardness to the low to mid $10^{15} n_{\text {eqv }}$ range. Successful operation (in a signal to noise sense) at the inner radii (mid $10^{15} n_{\text {eqv }}$ and greater) hinges on the exploitation of two newly uncovered gaps in the understanding of microstrip detector operation as presented in Section 3.3; violation of the NIEL hypothesis and the space charge limited avalanche (SCLA) effect.

In this section the ITk Strip sensor design's rich history is outlined in an overview of the progression of the silicon microstrip detector field towards the HL-LHC. The focus of the endeavour is to give the introduction and historical backdrop for the discussion of Strip sensor innovations of Section 4.3. 


\subsubsection{Historical Overview}

In the 1980s, the exhibition of $5 \mu \mathrm{m}$ tracking resolution with silicon strip detectors ignited much interest [48]. The focus in the field of high energy physics tracking began to shift from that of gas detectors to their higher granularity, more radiation resistant solid state counterparts. In the late $80 \mathrm{~s}$, the ground work for application to large scale experiments with sizable solid state surface coverage was being set [69]. Experience was building as larger and larger silicon detector components were installed in contemporary experiments such as LEP's DELPHI detector $\left(1.5 \mathrm{~m}^{2}\right.$ silicon Vertex detector). Meanwhile, more advanced quantitative models were being developed which deepened understanding of phenomena such as surface charge effects [58] and charge collection estimations [51]. With the onset of the 1990s R\&D campaigns for silicon microstrip detector implementation in the next generation of hadron colliders was underway. This mantle fell to the LHC as the American based Superconducting Super Collider was cancelled in 1993. Precious information $[21,53]$ was consolidated into the CERN program which had already formed dedicated R\&D collaborations.

Of cogent relevance to the ITk was RD2 [62,70,71] and associated work [61, 63]. The mandate of RD2 was to demonstrate the feasibility of a large area silicon tracker for the expected fluences of $O\left(10^{14} n_{\text {eqv }} \mathrm{cm}^{-2}\right)$. Large strides were made in the understanding of radiation damage, contributing strongly to the basics already discussed (see Section 3.4). This group concluded their studies in 1995, handing the torch off to RD48 [72]. RD48 was charged with ensuring the radiation hard designs were sufficient for the full intended LHC lifetime. This group contributed directly to the ITk Strip sensor design in its pioneering efforts to extend the radiation hardness of the contemporary technology [34,35,65,73]. In 2000, around the beginning of LHC installation, the RD48 collaboration was resolved giving way to its modern counterpart, RD50. Tasked with research of radiation hard silicon sensors for future collider experiments, with the initial focus on the HL-LHC, RD50 [23, 27,37, 52, 60, 74] and associated works $[33,35,36,59,75]$ are one of the two central players in the ITk Strip sensor development. The other main group, based in Japan, provided the direct lineage of the ITk design [4, 28, 44, 76-78]. The final ingredients for ITk Strip sensor prototyping was the observations of anomalous behaviour $[22,55]$ in the SCT sensors $[64,68,79,80]$.

By 2007, the sensor design had matured and the efforts of the two groups were joined giving birth to the modern ITk prototype line. Historically, short strip barrel 
prototypes; the ATLAS07 [25, 26, 29,39-41,45, 54, 81, 82], and the ATLAS12 [10, 24, 25, 38, 42, 67, 83]. Currently, the first true end-cap prototype, the ATLAS12EC [30] produced in 2016 (which incorporated a separate, European run, wedge shaped petalet stage $[32,43,84])$. And soon the first long-strip barrel sensor and final prototype expected before pre-production, the ATLAS17LS [31].

\subsubsection{Dawning of Radiation Hard Silicon Detectors}

The SCT R\&D in the late 90s provides the starting point for further discussion of the aspects specific to the radiation hardness of the ITk Strip sensor design. The harmful effects of type inversion in n-type bulk devices was well known and expected at the LHC fluences. Investigations into the less conventional $n^{+}-i n-n$ and p-bulk devices was being conducted in sync with campaigns on optimized $p-i n-n[34,64,76,79,80]$. These newer materials showed better radiation performance but were negated for use in the SCT due to lack of experience and availability in the industry at the time (p-type bulk devices) and cost (for the double sided process of the $n^{+}-i n-n$ ).

The optimization of material continued in Europe as focus shifted to the HL-LHC. RD48 which had recently confirmed the general applicability of the NIEL hypothesis, then confirmed its violation by discovering that high levels of interstitial oxygen in FZ silicon preferentially reduced proton induced radiation damage over neutron (via mechanisms of defect control). A technique for introduction of homogeneous oxygen content into $O(100 \mu \mathrm{m}) \mathrm{FZ}$ wafers was pioneered [65] allowing lower full depletion voltages at higher particle fluences. Radiation hardness was extended into the $10^{15}$ protons per square centimetre fluence regime. This offered the platform for optimization of semiconductor type and growth method [27,33-35,37,77]. Advantages of oxygen rich silicon was soon followed by the discovery of the SCLA effect an outcome of the lighter effective fields experienced by the electrons collected in an $n^{+}-i n-p$ scheme $[33,36,37,59,60]$. This surprise discovery offers advantages in signal gain that become endemic at high NIEL doses. With the current level of semiconductor radiation damage knowledge this advance is necessary for the ITk (Pixel detector) to function. [37,59,60]. Oxygen enriched silicon and SCLA are discussed further in Section 4.3.1.

Investigations into crystal growth and orientation on $n^{+}-i n-p$ radiation performance was examined by both groups in the early 2000s. The European RD50 collaborations also tested the variety against the oxygen enriched silicon. Extensive 


\begin{tabular}{|c|c|c|c|c|c|}
\hline \multicolumn{2}{|l|}{ Material } & Manufacturer & Symbol & $\rho[\Omega \mathrm{cm}]$ & $\mathrm{O}_{\mathrm{i}}\left[\mathrm{cm}^{-3}\right]$ \\
\hline \multirow{5}{*}{\multicolumn{2}{|c|}{$\begin{array}{l}\text { n- and p-type float zone } \\
\text { Diffusion oxygenated float zone } \\
\text { Czochralski } \\
\text { Magnetic Czochralski } \\
\text { Epitaxial layers on Cz-substrates }\end{array}$}} & Various & SFZ & $1-7 \times 10^{3}$ & $<5 \times 10^{16}$ \\
\hline & & Various & DOFZ & $1-7 \times 10^{3}$ & $\sim 1-2 \times 10^{17}$ \\
\hline & & Sumitomo, Japan & $\mathrm{Cz}$ & $\sim 1 \times 10^{3}$ & $\sim 8-9 \times 10^{17}$ \\
\hline & & Okmetic, Finland & $\mathrm{MCz}$ & $\sim 1 \times 10^{3}$ & $\sim 4-9 \times 10^{17}$ \\
\hline & & ITME, Warsaw & EPI & $50-100$ & $<1 \times 10^{17}$ \\
\hline & Wafer diameter (mm) & Wafer thickness $(\mu \mathrm{m})$ & Crystal growth & Crystal orientation & Resistivity ( $\mathrm{k} \Omega \mathrm{cm}$ ) \\
\hline p95 & 100 & 300 & FZ & $\langle 111\rangle$ & $\sim 6$ \\
\hline ATLASO5 & 100 & 300 & \multirow{2}{*}{$\begin{array}{l}\mathrm{FZ} \\
\mathrm{MCZ}\end{array}$} & $\langle 111\rangle$ & \multirow{2}{*}{$\begin{array}{l}\sim 6 \\
\sim 0.9\end{array}$} \\
\hline & 100 & 300 & & $\langle 100\rangle$ & \\
\hline \multirow[t]{3}{*}{ ATLASO6 } & 150 & 320 & FZ1 & $\langle 100\rangle$ & $\sim 6.7$ \\
\hline & 150 & 320 & FZ2 & $\langle 100\rangle$ & $\sim 6.2$ \\
\hline & 150 & 320 & MCZ & $\langle 100\rangle$ & $\sim 2.3$ \\
\hline \multirow[t]{2}{*}{ ATLAS07 } & 150 & 320 & \multirow{2}{*}{$\begin{array}{l}\text { FZ1 } \\
\text { FZ2 }\end{array}$} & $\langle 100\rangle$ & \multirow{2}{*}{$\begin{array}{l}\sim 6.7 \\
\sim 6.2\end{array}$} \\
\hline & 150 & 320 & & $\langle 100\rangle$ & \\
\hline
\end{tabular}

Figure 4.3: New silicon materials tested for the ITk. Top; the European RD50 Collaboration [27]. Bottom; the Japanese early ATLAS line [4].

surveys of FZ, oxygen enriched FZ, Czochralski, magentic Czochralski, and epitaxial silicon crystals were conducted by RD50, see the top table in Figure 4.3 [27,73]. Meanwhile, implemented in the earlier Japanese prototypes of the ATLAS05 to ATLAS07 was the baseline float zone as well as crystals grown by the magnetic Czochralski method. These samples are tabulated in the bottom table of Figure 4.3 [4,28,44,75]. Experience and the uncovering of SCLA in the oxygen enriched FZ curtailed further research into new material application at the ITk. The investigation of crystal orientation landed at the rational conclusion of $<100>$ in light of its smoother surface interface (see Figure 3.12).

\subsubsection{Genesis of a Large Area Radiation Hard Sensor Design}

The tracking of the ITk requires a hermetic detector. The radiation levels, a solid state one. The sensor layout must be optimized for the HL-LHC's instantaneous and integrated luminosity guidelines. And, it must feature the largest silicon surfaces of any microstrip detector in history to make the ITk cost-effective. This leaves it grossly susceptible to the instability inherent in the surface. There, the delicate interplay of charges at the $\mathrm{Si}-\mathrm{SiO}_{2}$ interface with those in the bulk and at the outer surface of the sensor (mobile and immobile) is coupled with the increased field gradient at the shaped junctions. This makes it one of the most sensitive parts of the detector. As seen (Section 3.4.2) this is only amplified by the damage caused by years 
of irradiation with particles of the highest energy synthetically attainable. A cuttingedge $n^{+}-i n-p$ design was developed featuring new and advanced radiation hard strip isolation, and PTP structures. The reduction of the dead area in the detector, directly at the sensor level, was similarly advanced by the ITk collaboration. Slim edges, nearly half the width of conventional, are introduced as is the stereo annulus geometry.

The current prototype sensor is the seventh in a noble line that traces its way back to the first 'large' area p-type microstrip device considered by the ATLAS collaboration. The "p95" sensor design, fabricated in 1995-96, was part of the SCT R\&D [76]. It was a high resistivity, $6 \mathrm{~cm} \times 3.4 \mathrm{~cm}$ barrel sensor with $50 \mu \mathrm{m}$ pitch. Conventional p-stops of two dopant levels were used (Figure 4.3) and the sensors were irradiated with the $24 G e V$ protons of the CERN PS IRRADiation facility to fluences of 0.43 and $1.1 \times 10^{14} \mathrm{pcm}^{-2}$. The p95 showed the viability of p-type bulk in high radiation environments and was revisited as tests began on the early ATLAS line [4,77].

The ATLAS05 and ATLAS06 (A05 and A06) designs began the development of strip isolation structure necessary for ITk fluences. This was done in concert with the investigation of crystal already discussed. Newly developed isolation structures [78] as well as p-stop, p-spray, and combinations were considered in various configurations and at various doping concentrations (see Figures 4.3 and 4.14). The A06 marked the transition from the four inch wafer to a six inch wafer process with a doubling in sensor area. The roughly $6 \mathrm{~cm}$ square A05 expanding to the $12.4 \mathrm{~cm} \times 6.3 \mathrm{~cm}$ A06 (see wafer layouts in Figure 4.4). Both submissions (and those following) also included roughly $1 \mathrm{~cm}$ square minis as comparable irradiation test samples for the various structures. The A05 and A06 mini-sensor irradiation up to $2 \times 10^{15} n_{\text {eqv }} \mathrm{cm}^{-2}$ at the CYRIC 70MeV proton facility was accompanied by extensive testing [4, 28,44,75].

The FZ grown ATLAS07 (A07) at $9.754 \mathrm{~cm}$ square was the first true ITk prototype as the first iteration of short strip barrel sensor [29,81]. As seen in Figure 4.4 the full-size sensor implements four rows of strips in two configurations, axial (parallel to sensor edge) and stereo (rotated by $40 \mathrm{mrad}$ ). The A07 continued the strip isolation investigations using the front-running structure optimized by technology-CAD (TCAD) [85] simulation in its full-size sensors [54]. This was incorporated into the $74.5 \mu \mathrm{m}$ pitch with the 1280 readout strips per row associated with the current line of readout chip. The two field shaping strips were also used in the ATLAS07 bringing the total number of strips up to 1282 per segment in the barrel. 


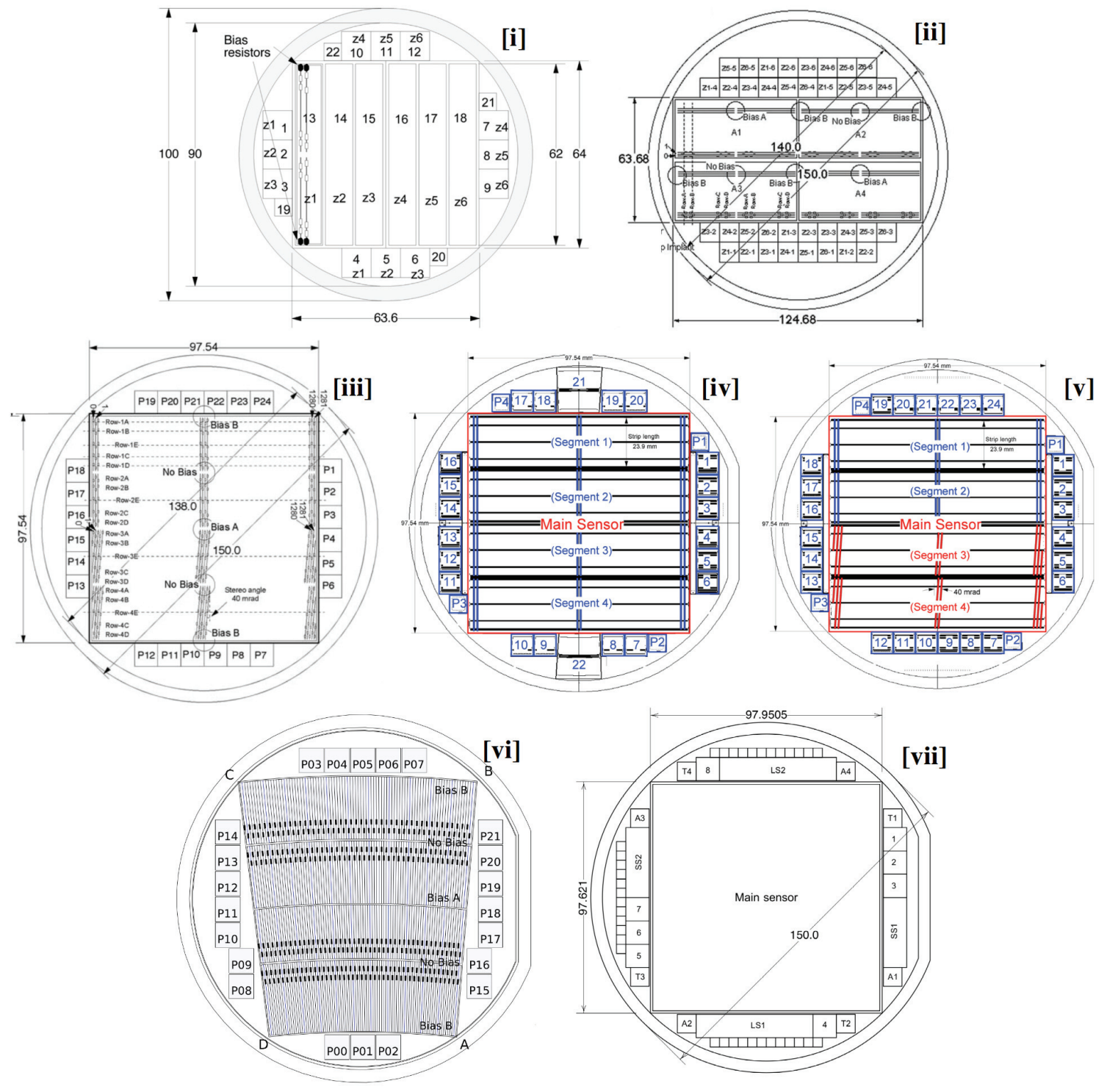

Figure 4.4: Wafer layouts of the ATLAS ITk Strip prototype sensors. [i] ATLAS05 [28] [ii] ATLAS06 [4], [iii] ATLAS07 [29], [iv] ATLAS12A (axial) [10] [v] ATLAS12M (stereo) [10] [vi] ATLAS12EC [30] [vii] ATLAS17LS [31] 
The large size did not come without a learning curve as design issues with the isolation structure were uncovered via the IR illumination of microdischarge [29]. The mask was successfully adjusted in a second series of A07. A final round of strip isolation structures were considered and the development of an optimized punch through protection began in the minis (see Figures 4.14 and 4.18). The A07 were irradiated up to 1.3 and $1.0 \times 10^{15} n_{\text {eqv }} \mathrm{cm}^{-2}$ with CYRIC protons in Japan and reactor neutrons from the TRIGA reactor in Slovenia. Dedicated studies of the surface and bulk damage were conducted $[4,26,39,40,82]$ as were larger statistic studies of the unirradiated sensors [45].

At the same time as the A07 campaigns was the submission of small batches of dedicated test sensors focused on optimization of the edge structures $(4 \mathrm{~mm} \times 4 \mathrm{~mm}$ diodes $)$ and PTP $(1 \mathrm{~cm} \times 1 \mathrm{~cm}$ minis $)$. In terms of the edge, reduction of dead space was the foremost goal and was studied in concert with the effect of guard ring size, type, and multiplicity $[40,41,82]$. The PTP minis implemented further optimized structures and saw the introduction of the radiation hard gated design [10,38,40,41,82]. These features are discussed further in Section 4.3.2.

The short strip barrel design was hardened in its current formulation; the ATLAS12 (A12) [10]. Two sets of the sensor exist. The "Main" variant (A12M) utilizing a similar half stereo strip design as the A07 and a fully axial version called "Axial" (A12A) (Figure 4.4). The leading PTP option of the test sensor batch was improved by an extension of the TCAD modelling [38] to the entirely novel "full-gate" structure and implemented in the full size sensor. The A07 p-stop configuration is used with an identical number of strips and strip pitch. A single $n^{+}$guard ring with extended $p^{+}$ edge implant incorporating two metal structures was used to allow evaluation of the slim edge $(450 \mu \mathrm{m})$ against a more conventional one $(910 \mu \mathrm{m})$. For TCAD simulation verification further PTP structures were implemented in the A12 minis. Preparations for the first endcap prototype continued as stereo strips were investigated in minis with square and wedge shaped active areas [83]. The A12 minis have also been used to investigate the evolution of Lorentz angle with neutron irradiation up to magnetic field strengths of $1 T$ using the $5 \mathrm{GeV}$ electron beam at DESY in Hamburg, Germany [1].

Over 160 ATLAS12 have been produced (one third with slim cut) and tested in large numbers [42]. The mature design, in its sixth iteration, resulted in excellent IV and CV character (Figures 5.15 and 5.18) despite a slightly lower resistivity than 

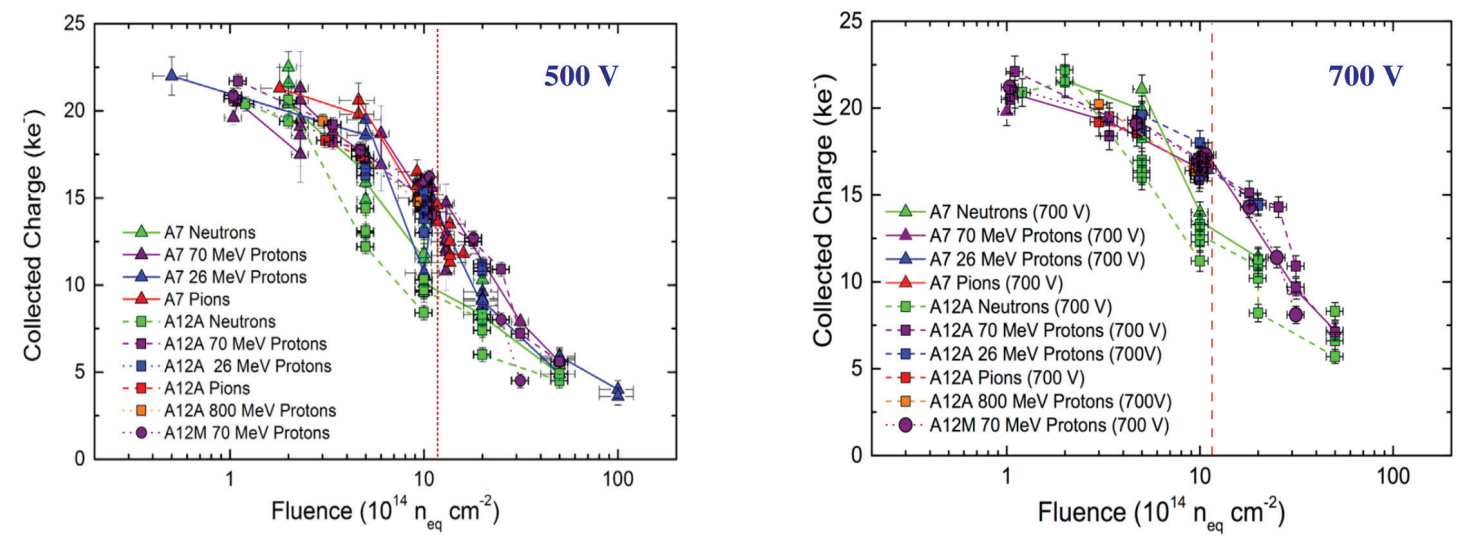

Figure 4.5: Charge collection of short strip barrel prototypes as a function of irradiation dose and type. Left; nominal operating voltage in the ITk, $500 \mathrm{~V}$ [25]. Right; maximal operating voltage, $700 \mathrm{~V}$ [1]. Beneficial annealing of $10 \mathrm{~min}$ at $80^{\circ} \mathrm{C}$ conducted. Red lines indicate Strip detector's maximum expected fluence.

expected for the ITk. A07, A12A, and A12M sensors have undergone extensive irradiation campaigns up to $10^{16} n_{e q v} \mathrm{~cm}^{-2}$ with pions $\left(300 \mathrm{MeV}\right.$ at PSI), gammas $\left(\mathrm{Co}^{60}\right.$ at BNL), neutrons (TRIGA reactor) and protons $(23,27,70$, and $800 \mathrm{MeV}$ protons at KIT, Birmingham, CYRIC, and Los Alamos). Extensive testing of the bulk and surface structures [24,25] as well as the stereo strips [83] have satisfied specifications. Signal to noise ratios sufficient for full life operation in the ITk have likewise been shown via testbeam and beta source measurements. This has been seen in Section 2.7 and can be seen, more generally, in Figure 4.5 which shows the collected charge as a function of NIEL fluence for different particle types [1,25]. As discussed in Sections 2.7 and 3.3.7 the noise at the front-end will be stable and on the order of $500 e^{-}$.

Successful operation in the harsh ITk conditions has been exemplified in the A07 and A12 (see a large number of presented results including Figures 2.14, 3.20, and $4.5)$ but there remains two hurdles before the project can be considered ready for production. The first and more challenging of these is a full-size stereo annulus sensor with slim-edge design. The second, is a barrel module with the double length $(\sim 4.8 \mathrm{~cm})$ strips that will be used in the outer two barrel layers.

The ATLAS12EC (A12EC) is the most recent ITk Strip sensor iteration, produced in late 2016 [30]. It is a full-size R0 prototype. With an area of approximately $90 \mathrm{~cm}^{2}$ it is one of the largest of the stereo annulus sesnor designs (see Figure 4.4 and 

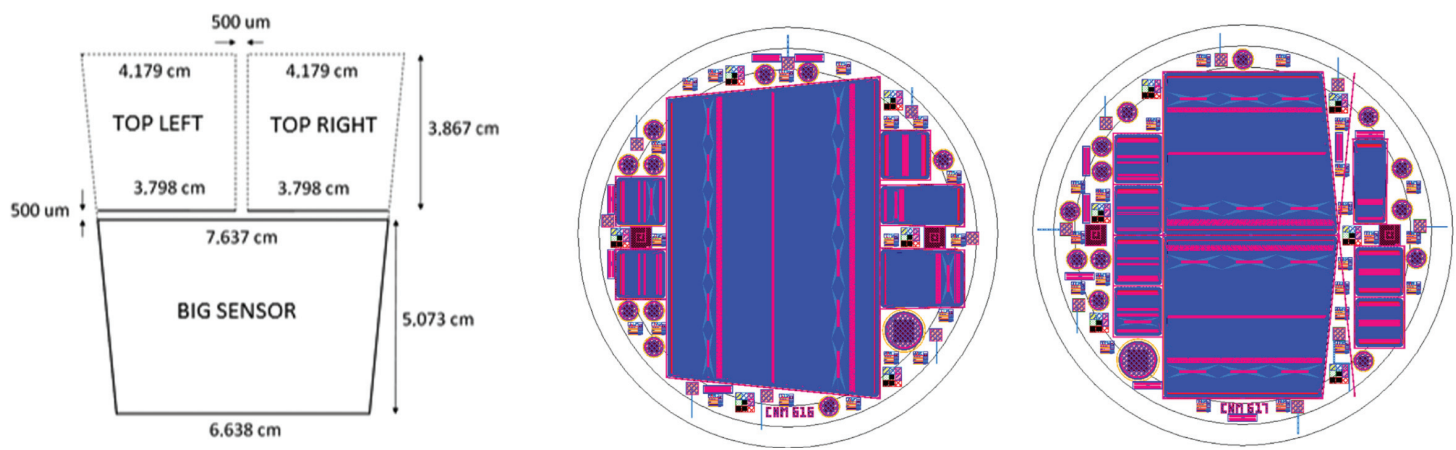

Figure 4.6: The sensors of the petalet program. Left; schematic showing the petalet structure and sizes. Center; wafer layout for big sensor. Right; wafer layout for top sensors. [32]

Section 4.1.2) and the first one ever fabricated. The surface layout is very similar to that of the production sensors overviewed in Section 4.1.2. It implements the p-stop and PTP structures that were optimized in the preceding sensor submissions. Of utmost importance will be to see the effect of this new geometry on stability and radiation hardness. The beginnings of this important analysis is within the scope of this Master's thesis work and is discussed in Chapter 5.

Although a descendent in the ATLAS lineage the A12EC benefited from the parallel development of the European "petalet" project [32,43,84]. The petalet was a three sensor wedge shaped miniature petal (see Figure 4.6) fabricated to test the fan shaped geometry and stereo strips. While primarily offering a realistic test device for petal design development, the petalet sensors did offer one peculiarity in the design of "embedded pitch adapters" [43]. Introduction of a second metal layer at the surface to adapt the fan nature of the strips to the linear pads on the readout chip.

The final prototype expected before production is the ATLAS17LS (A17LS) [31]. The design of this sensor is in the final preparatory state and sensors are expected in 2019. It will feature oxygenated float-zone silicon as well as the two segments with $4.8 \mathrm{~cm}$ strips indicative of the long strip barrel sensor.

\subsection{Innovations of the ITk Strip Sensor Design}

The radiation damage at HL-LHC fluences is unlike that at lower orders of magnitude. As the value of $10^{15} n_{e q v} \mathrm{~cm}^{-2}$ is surpassed the build-up of defects and their induced 
trapping effects begin to overwhelm the initial bulk characteristics (see Figure 4.9). Inexperience prior to the HL-LHC project with these fluences and the related use of $p^{+}-i n-n$ type devices has lead to pioneering efforts and discoveries that are endemic in the ITk sensor development. These are advances required for stable operation in this new high radiation regime and are detailed in this section.

\subsubsection{Radiation Hardness in the Bulk Material}

Investigations into the radiation hardening of silicon detectors by the CERN RD48 and RD50 collaborations was happily rewarded. Two effects that were unexpected with the contemporary knowledge of the microstrip devices and radiation damage were uncovered; means for controlling defect formation mechanisms with interstitial oxygen, and the space charge limited avalanche (SCLA) effect. Without these critical advancements the charge collection properties of the sensors after irradiation may have been significantly degraded to the point of an insufficient signal to noise ratio in several places in the ITk detector [59].

\section{Defect Engineering; Oxygen Enrichment}

The physics of irradiated microstrip detectors is dominated by defects. As such, much effort has been spent on defect identification and characterization by the aforementioned legacy of RD collaborations. One such related effort that has seen success is defect engineering; deliberate addition of impurities to induce beneficial effects. In the RD48 era, investigations into the enrichment of float-zone silicon to increase the interstitial oxygen content bore fruit. It was shown that an increase of two orders of magnitude in the oxygen concentration, from the order $10^{15} \mathrm{~cm}^{-3}$ seen in normal $\mathrm{FZ}$ methods, could provide a reduction in the effect of radiation damage on the effective doping concentration by a factor of three [34].

The mechanism considered responsible for the advantage is the nature of interstitial oxygen to act as a sink for single vacancies $[23,27,65]$. The resulting shallow $V-O$ defect is formed in opposition to divacancies and their associated deeper traps (such as $\mathrm{V}_{2} \mathrm{O}$ ). With less deep traps being formed less negative space charge builds up in the bulk. The end effect is a reduction in the degradation of the full depletion voltage with fluence. This is seen directly in Figure 4.7 with p-type bulk. Since it is the removal of single vacancies that is responsible for this advantage this effect is predominant in charged particle irradiation (violating the NIEL hypothesis). The 


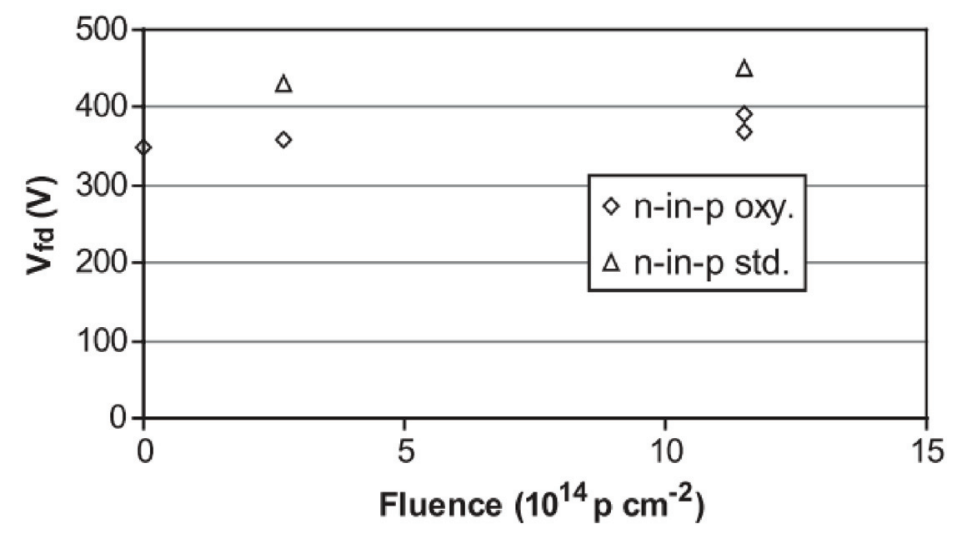

Figure 4.7: Advantage of oxygen enriched silicon in high radiation environments. Full depletion voltage as a function of proton fluence up to $1.1 \times 10^{15} \mathrm{pcm}^{-2}$ for oxygen enriched and un-enriched p-type bulk minis. [33]

oxygen enrichment also proved to have no effects on the wafer resistivity, making it applicable for the low leakage requirements of the ITk [34,65]. This is seen in Figure 4.8. With the importance of the oxygen enrichment related to charged particle fluence the NIEL $1 \mathrm{MeV}$ proton equivalent is a relevant unit, with a weighting factor of approximately $1.67 n_{\text {eqv }}$ (ie. $1 p_{\text {eqv }}=1.67 n_{\text {eqv }}$ ) [59].

The technique used today for homogeneous oxygen enrichment of FZ was developed by RD48 in 1999 [65]. It involves thermal diffusion through a $\mathrm{SiO}_{2}$ layer on all faces of the pure silicon bulk. The oxide is later removed by polishing. The method was implemented in n-type bulk at the same time as the group was developing their first p-type sensor [34]. It was subsequently implemented by RD50 in a batch of $1 \mathrm{~cm}$ square mini-sensors of p-type bulk, some of which were left un-enriched for comparison $[33,36,59]$. The mini-sensors were irradiated with $23 \mathrm{GeVp} p^{+}$to fluences of $1.1,3.5$, and $7.5 \times 10^{15} p_{\text {eqv }} \mathrm{cm}^{-2}$. The studies of these two batches of sensors proved, undoubtedly, the superiority of $n^{+}-i n-p$ over $p-i n-n$ and that of oxygen enrichment over the un-enriched version. This is shown via charge collection measurements in Figure 4.9 .

\section{The Space Charge Limited Avalance Effect}

In 2006 long-term annealing studies of the oxygen-enriched mini $n^{+}-i n-p$ sensors was concluding and bore another round of good tidings for radiation hard operation [36]. 


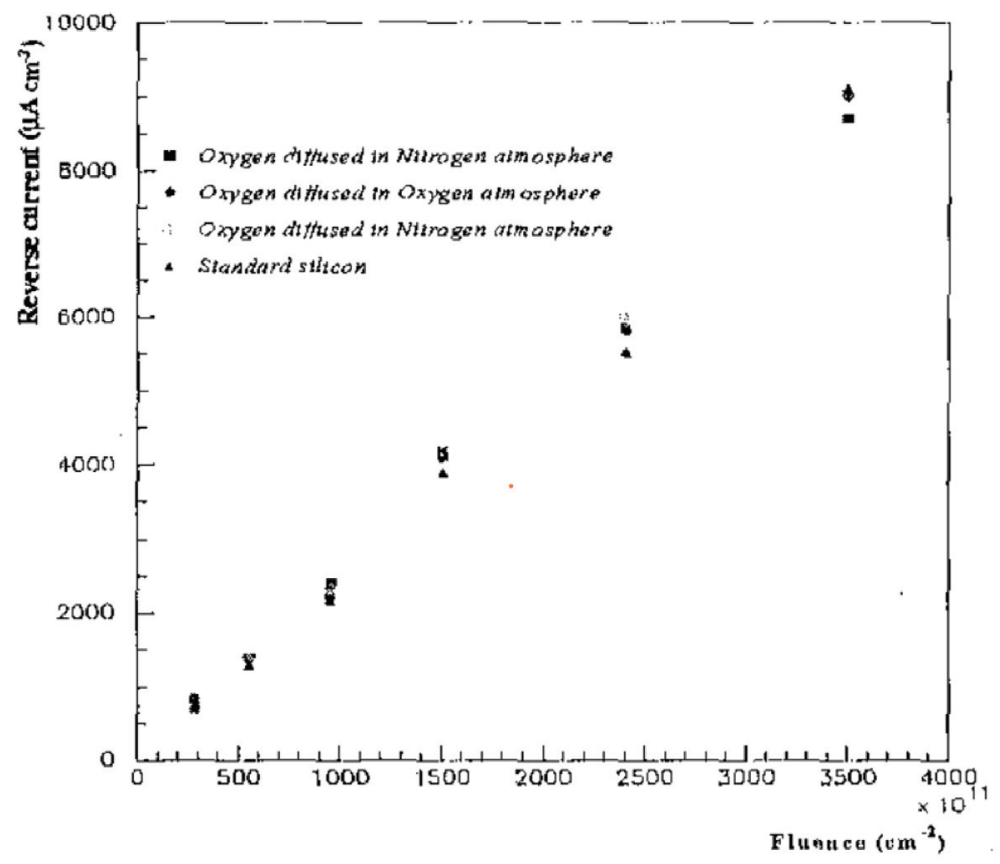

Figure 4.8: The effect of oxygen enrichment on silicon resistivity. Sensor leakage current as a function of fluence for oxygen enriched and un-enriched n-type bulk sensors of size $6.4 \mathrm{~cm}$ square (SCT masks). Samples with different enrichment procedure atmospheres are shown. [34]
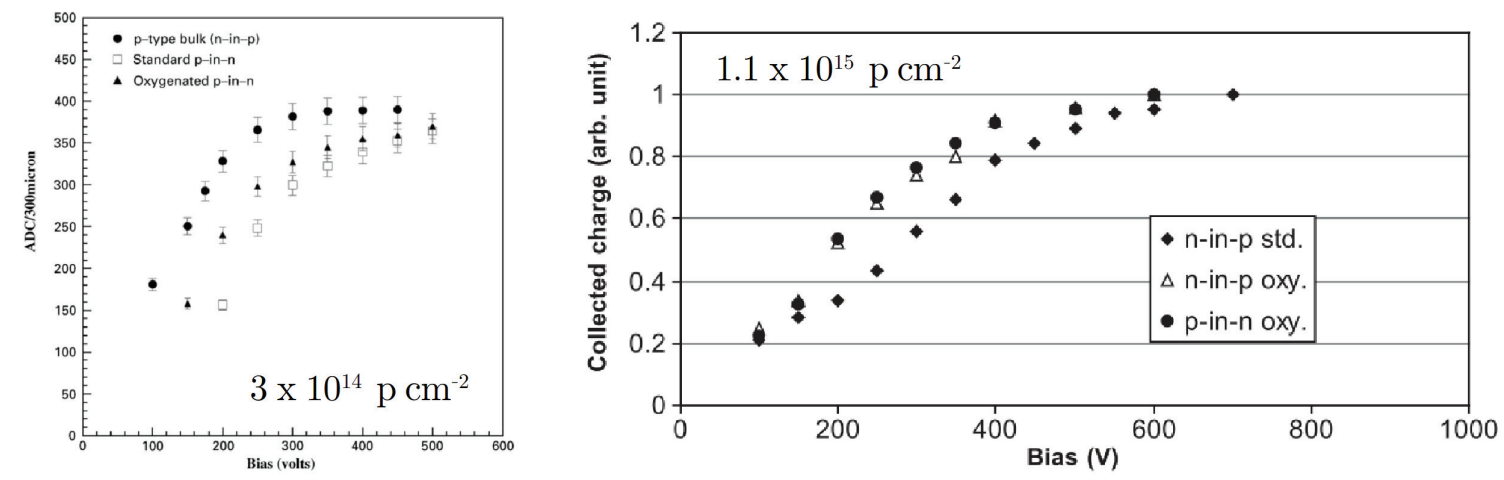

Figure 4.9: Radiation hardness superiority of the oxygen enriched $n^{+}-i n-p$ bulk material. Left; at lower proton fluences $\left(3 \times 10^{14} \mathrm{pcm}^{-2}\right)$ the type advantage of p-bulk [35]. Right; at higher proton fluences $\left(1.1 \times 10^{15} \mathrm{pcm}^{-2}\right)$ the advantage of enriched interstitial oxygen content [33]. y-axis units can be considered as arbitrary. 


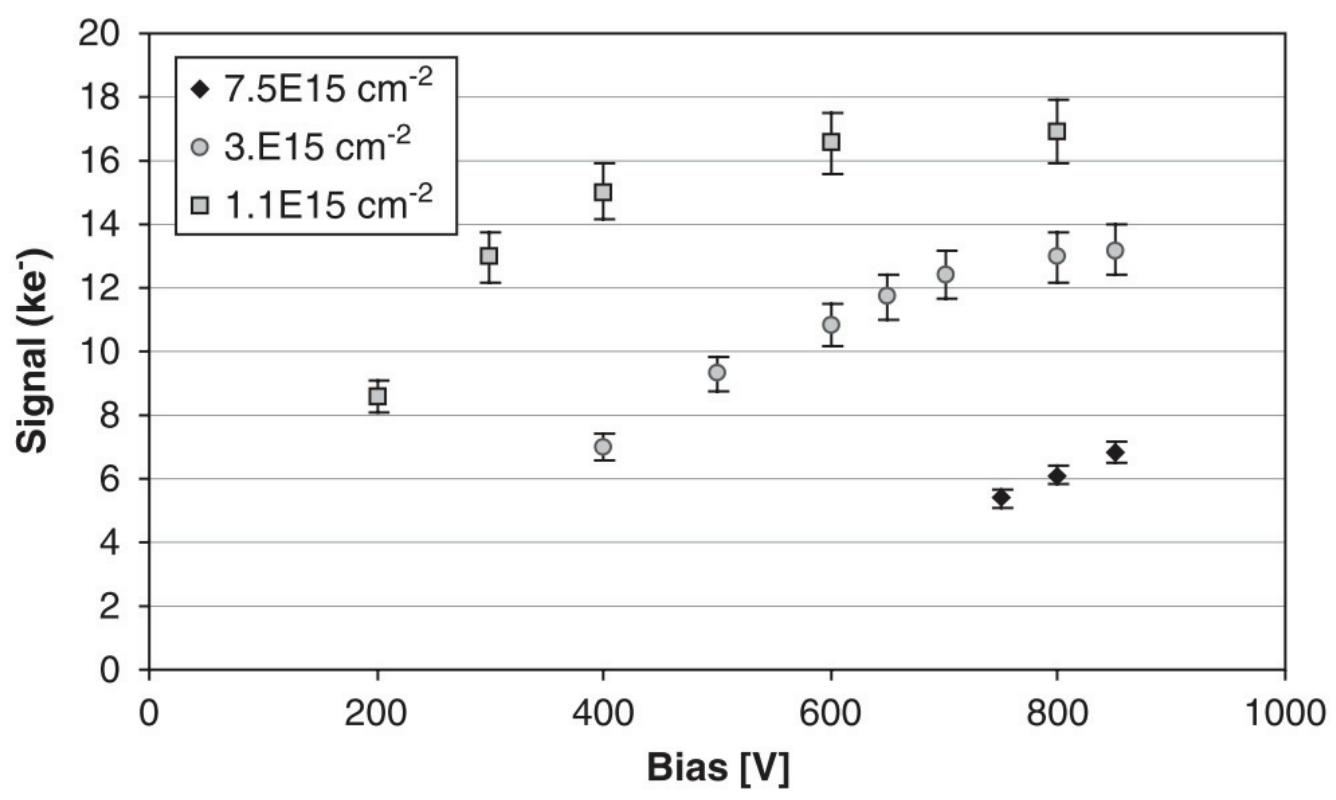

Figure 4.10: Charge collection versus bias voltage for p-type mini sensors at three fluences of proton irradiation (as seen). Values are normalized to the preirradiation signal size.

Despite the advancements offered by the oxygen enrichment process the eventual overwhelming of effective dopant concentration by deep trapping sites in the bulk is inevitable. Introduced into the silicon bulk they begin to eat away at the signal (by trapping). A large decrease in the collected charge is observed as the proton fluence begins to climb to $7.5 \times 10^{15} p_{\text {eqv }} \mathrm{cm}^{-2}\left(\simeq 4.5 \times 10^{15} n_{\text {eqv }} \mathrm{cm}^{-2}\right)$ as shown in Figure 4.10 [59]. Unfortunately, the expectations gained from n-type irradiation campaigns on the long term annealing properties of the sensors did not bode well. Extension of annealing curves such as Figure 3.22 saw an exponential like saturation of the reverse annealing effect (and thus $V_{f d}$ ) at higher levels (even in the oxygenated n-type case). The concept of silicon at the inner radii of an HL-LHC experiment was under jeopardy and along with it the fate of the ITk project [59].

A newly discovered phenomena associated with the collection of electrons after heavy irradiation provided the saving grace. As shown in Figure 4.11 after higher fluences the charge collection efficiency was found to start increasing after an annealing period of roughly 1500 days at $20^{\circ} \mathrm{C}$ (or $300 \mathrm{~min}$ at $80^{\circ} \mathrm{C}$ as seen in Figure 4.11 ). Anomalously, this was the second flat portion of the annealing curves (Figure 3.22) 

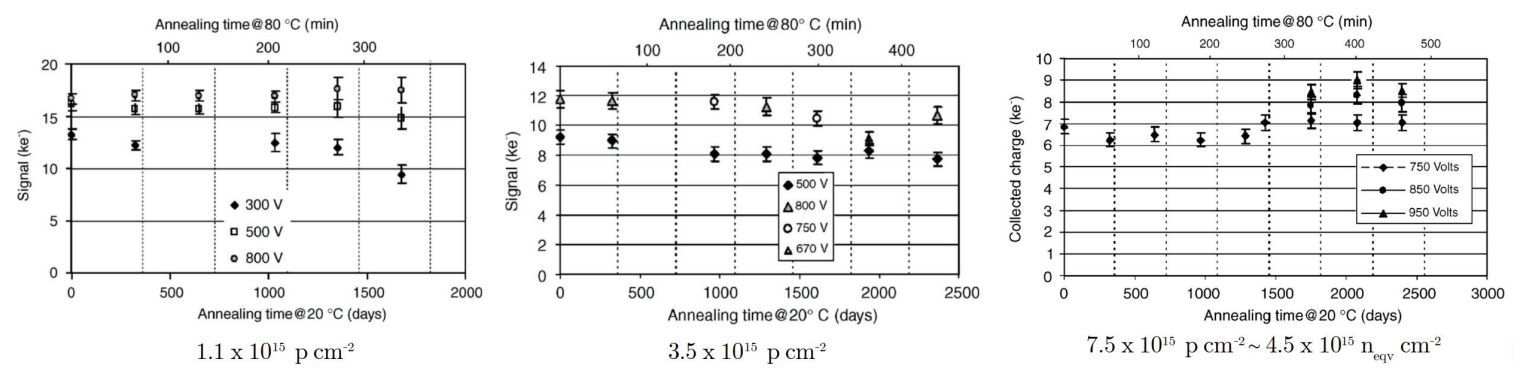

Figure 4.11: SCLA effects in early ITk Strip prototype mini sensors. Annealing curves after $23 \mathrm{MeV}$ proton irradiation to thefluences of $1.1 \times 10^{15} \mathrm{pcm}^{-2}$ (left), $3.5 \times 10^{15} \mathrm{pcm}^{-2}$ (left), and $7.5 \times 10^{15} \mathrm{pcm}^{-2}$ (left). The SCLA effect is seen at the higher fluence. [36]

and CV measurements confirmed that the annealing of effective doping concentration was not the cause. The answer was found in the regions near the implants filled with trapping sites and high field gradients as well as the higher likelihood for impact ionization offered by the quick moving electrons being collected there. The negative space charge build up increases the high field gradients which eventually causes the electrons to avalanche and increases the signal. The quenching mechanism which inhibits breakdown is in the concentration of donor traps near the implants. These are introduced by both electromagnetic and collisional defects (see Section 3.4). They act as deep hole traps that capture the holes released in the ionizing avalanche, nullifying the negative space charge increase and stabilizing the field. [36, 59,60]

Subsequent experiments carried out by RD50 [37] show that this effect extends to the higher fluences that will begin to accumulate towards the ITk end of lifetime. In that radiation regime the effect begins to dominate after sufficient annealing allowing the signal to climb past its unirradiated value. This is seen in Figure 4.12 showing typical fluences expected in the ITk Strip and Pixel detectors.

The benefits of space charge limited avalanche have been seen in the ATLAS12 large area sensors [25]. This is seen in the right side of Figure 4.13 with an ATLAS12M irradiated to $1 \times 10^{15} n_{e q v} \mathrm{~cm}^{-2}$ with $70 \mathrm{MeV}$ protons. The marked quadratic increase and decrease of collected charge indicative of the beneficial annealing of full depletion voltage is very evident in the lower voltages. However at the higher voltages the curve does not follow the trend of effective doping concentration as SCLA begins to take effect. Interestingly, the SCLA effect is not observed in the stereo-strip-withholding 

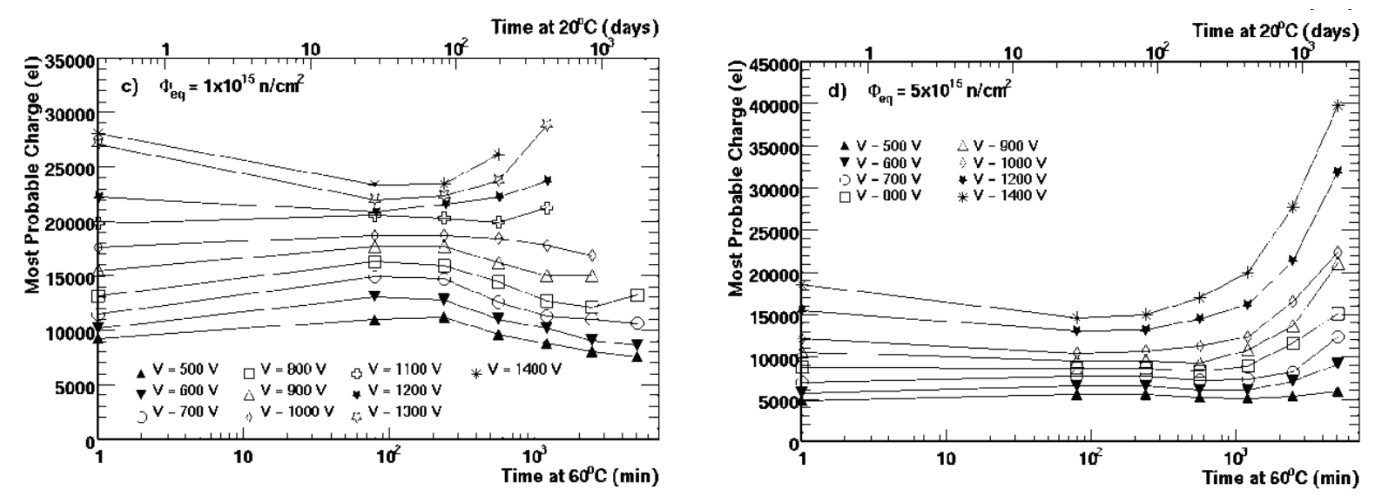

Figure 4.12: The benefits of space charge limited avalanche. Most probable charge (mean of the charge collection spectrum) as a function of fluence and bias voltage. Left; roughly the maximum fluence expectation for the ITk Strip detector. Right; more typical fluence expectation in the ITk Pixels detector. [37]

A12A sensors irradiated to the same dose. During experimental operation the SCLA annealing regimen is incorporated into the ATLAS maintenance shutdowns and will allow exceptional end-of-lifetime operability of the detector.

\subsubsection{Optimization of Surface Layout}

The ATLAS ITk Strip sensor design is a pioneer in the field of microstrip detectors boasting a number of unique features. Development has gone into two main aspects; minimization of dead space in the detector and optimization of surface structures for high fluence doses. Large advances have been made with implementation of the stereo annulus geometry and slim-edge designs as well as novel strip isolation and punch through protection structures. The design, currently in its seventh iteration, has shown great promise in the barrel geometry (see Section 4.2.3).

\section{Strip Isolation Structure}

The physics at the surface of microstrip detectors revolves around the charge at the interface of the integrated capacitor and the silicon. As seen, it is a complex environment with many factors affecting the magnitude and distribution of this concentration. Its dynamic nature, interface to the outside world, and proximity to the implant's high electric field gradients make it especially sensitive. In the $n^{+}-i n-p$ type sensor the effects reinforce the interface charge and its mirror charge collection in 

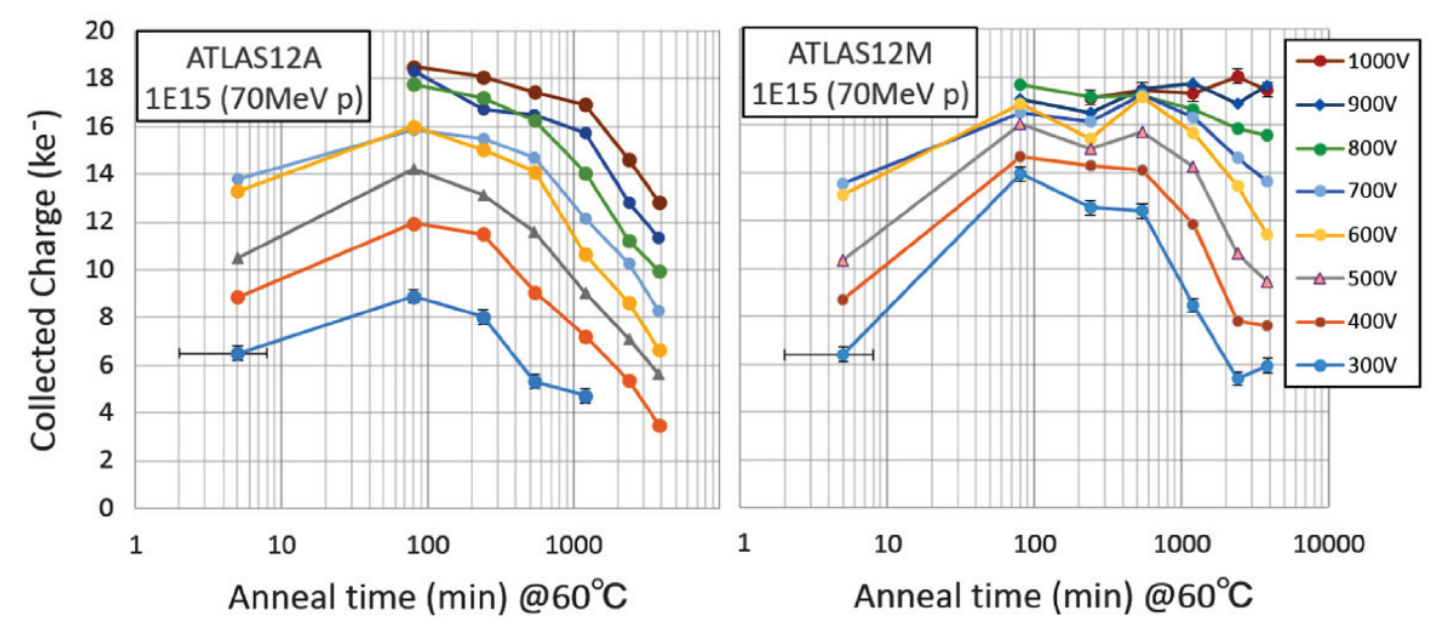

Figure 4.13: SCLA effects in full size ITk Strip short strip barrel sensor prototypes.Left, the A12 Axial. Right, the sterero strip containing A12 Main. [25]

the silicon bulk leading to short-circuiting of the implants to one another via the accumulation layer of electrons. Thus, for sensors of this type a strip isolation structure is of absolute necessity.

After the huge ionizing dose in the ITk, up to 50MRad, the surface charge concentration will rise by two orders of magnitude from the $10^{10} \mathrm{~cm}^{-2}$ typical of $<100>$ silicon [38]. This will result in an increased density in the electron accumulation layer which will increase the electric fields in the nearby volume and decrease the interstrip resistance. In fact the strip isolation can be quite easily obtained if the isolation structure dopant level is high enough (see below) and it is the consideration of minimizing electric field near the surface that drives the surface development. An upper limit of allowable field strength in the design is given by the silicon avalanche breakdown value, $\sim 300 \mathrm{kV} \mathrm{cm}^{-1}$.

A large number of isolation structures were tested, they are summarized in Figure 4.14 and Table 4.3. These included novel designs, such as the DC field plate seen in the Zone 05 of the ATLAS05 (Figure 4.14 top) [78]. The initial results, Figure 4.17 left, showed that all structures performed quite well and further investigation into the common p-stop was done for the full-size sensor in later generations. Results for these later generation prototypes after irradiation have already been seen (Figures 3.19 and 3.20). 


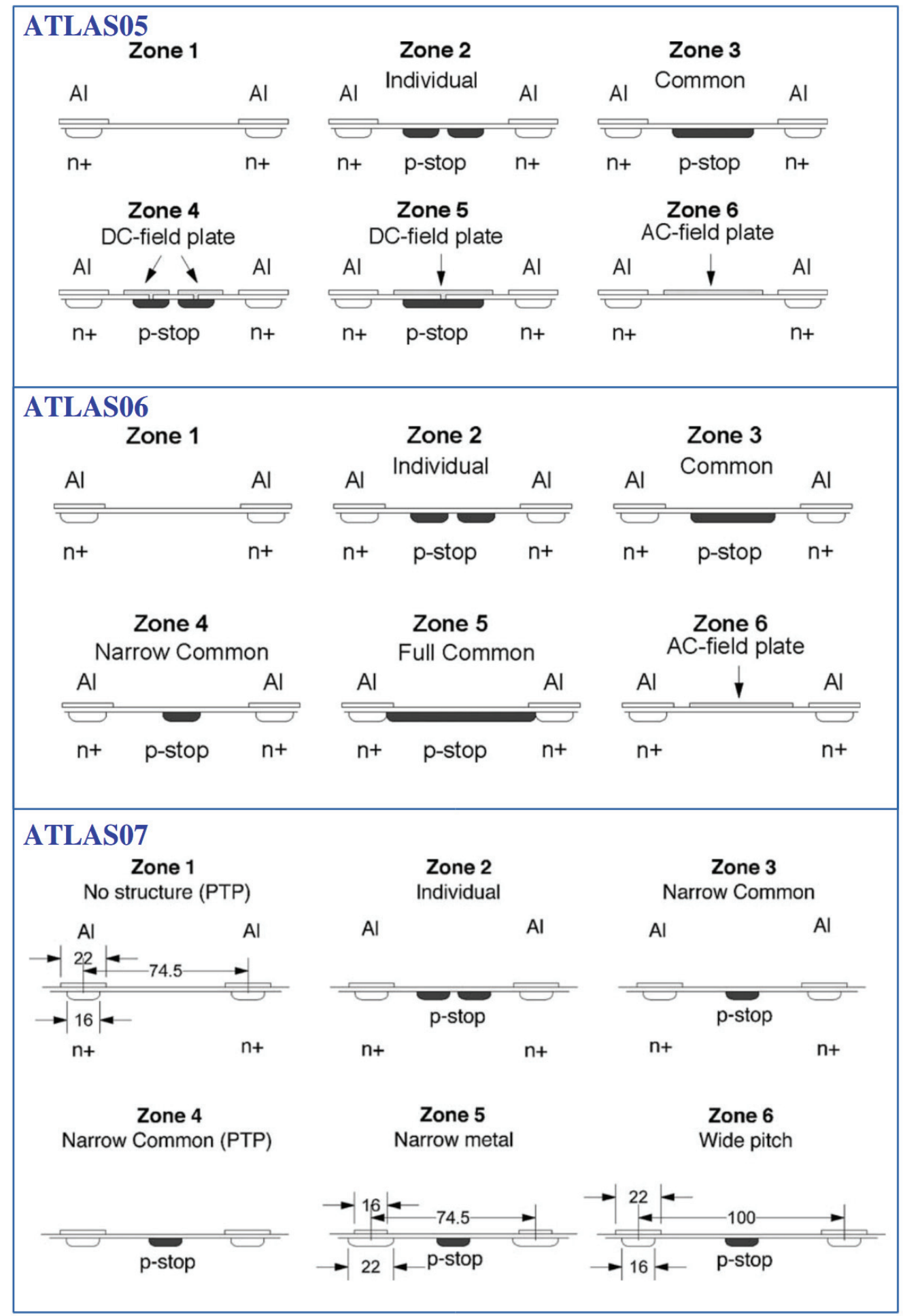

Figure 4.14: Strip isolation structures of the ATLAS05 (left), ATLAS06 (center), and ATLAS07 (right) prototype sensors. [4] 


\begin{tabular}{llll}
\hline & p-Stop & p-Spray+p-stop & p-Spray \\
\hline p95 & $2 \times 10^{13}, 1 \times 10^{14}$ & - & - \\
ATLAS05 & $1 \times 10^{13}, 2 \times 10^{13}$ & $2 \times 10^{12}+2 \times 10^{12}$ & $2 \times 10^{12}$ \\
ATLAS06 & $5 \times 10^{12}, 1 \times 10^{13}, 2 \times 10^{13}$ & $2 \times 10^{12}+2 \times 10^{12}$ & $2 \times 10^{12}$ \\
ATLAS07 & $2 \times 10^{12}, 4 \times 10^{12}$ & $2 \times 10^{12}+2 \times 10^{12}$ & $2 \times 10^{12}$ \\
\hline
\end{tabular}

Table 4.3: Dopant levels of the strip isolation structures tested in the A05 to A07 sensors. [4]

The A07 common p-stop was optimized by a data-verified TCAD simulation $[38,54,85]$. This allowed the electric potential and field distributions to be calculated across the volume. Figure 4.15 shows the calculated electric potential in the bulk of an ATLAS07 simulation at $200 \mathrm{~V}$ reverse bias. It was found that over the course of irradiation the high field location shifts from the implant edge to that of the p-stop. This is seen in Figure 4.16 left. This is one of the reasons for the use of overhanging strip metal at the implants, which are required to contain the field and reduce capacitive coupling between strips. It also reinforces the crucial need to minimize the electric potential at this point in particular. The magnitude and proportion of the peaks at the implant and p-stop was determined by TCAD simulation to be correlated most closely with the distance between the two structures (preferring wider) also showing dependency on the p-stop width (narrower), offset (less symmetric), and multiplicity (single) [54]. Figure 4.16 right displays a TCAD electric potential profile across the interstrip region for the implant-gap comparison. This also supports the movement to a smaller implant width (and thus larger distance from implant to p-stop). A telling comparison is that of the p-stop structures on the ATLAS07 sensors (Figure 4.14), shown in the centre and right plots of Figure 4.17.

\section{Punch Through Protection (PTP)}

The sensor is a delicate electrical device that is put in a harsh environment very near to a high intensity beam of $7 \mathrm{TeV}$ protons. With expectations for 10-15 years operation, fail-safes must be in place in the event of undesired beam operation. In the sensors the uncontrolled splash of the beam's high energy particles can be devastating. The large charge liberation generates a big voltage pulse at the implants which can 


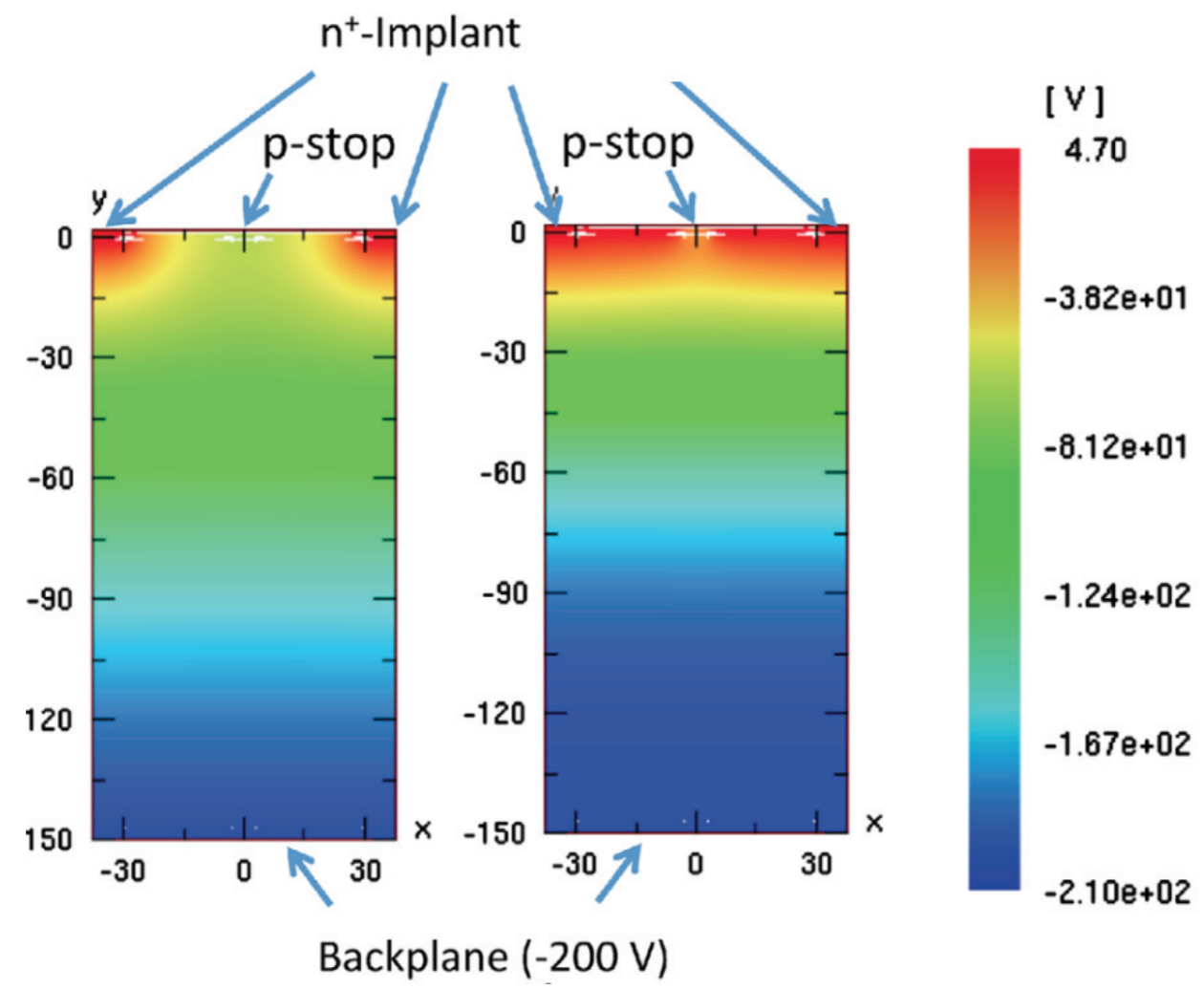

Figure 4.15: Electric potential distribution in unirradiated (left) and irradiated (right) TCAD simulated ATLAS07 sensors. Increased field gradient at the surface and reduced depletion depth are observed in the irradiated sample. [4]
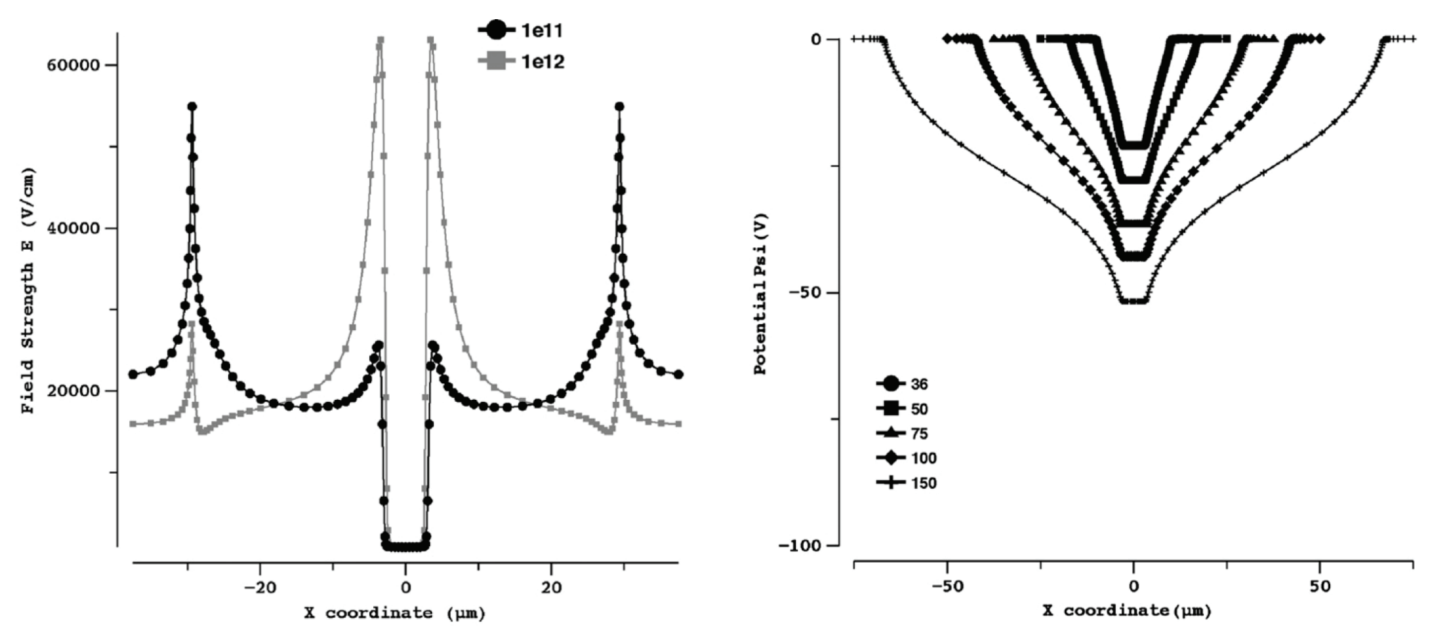

Figure 4.16: TCAD simulations for p-stop optimization. Left; electric field strength as a function of distance from the centre of the p-stop. Two interface charge densities are shown with the unit $\mathrm{cm}^{-2}$. Right; electric potential for $6 \mu \mathrm{m}$ p-stop with varying strip pitch in micrometres, interface charge of $1 \times 10^{12} \mathrm{~cm}^{-2}$. [38]. 

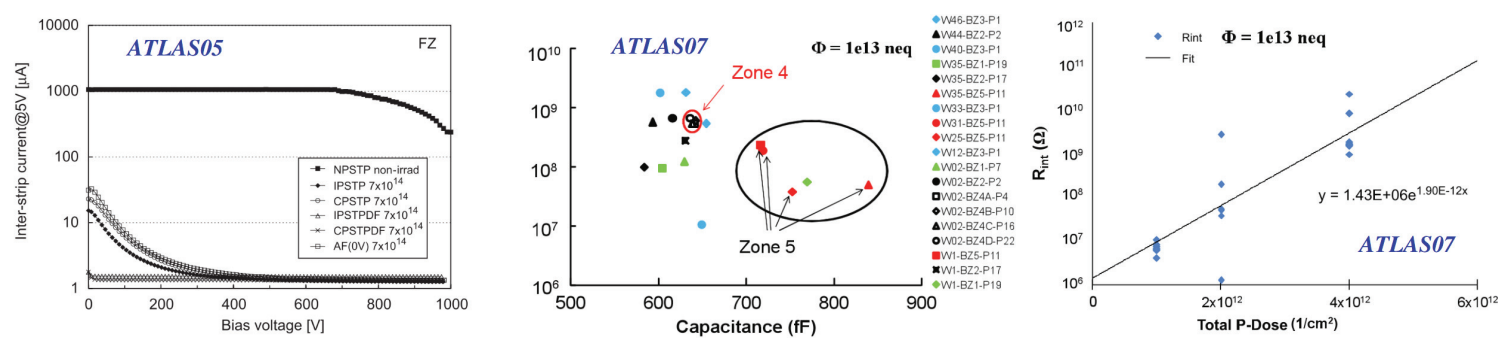

Figure 4.17: Development of a radiation hard $n^{+}-i n-p$ strip isolation structure. Left; A05 interstrip currents as a function of bias, structures as given in order of Figure 4.17 top [28]. Centre; interstrip resistance vs. capacitance for the A07 structures (Figure 4.17 bottom) irradiated to $1 \times 10^{13} n_{e q v} \mathrm{~cm}^{-2}$. Right; for the same irradiation interstrip resistance as a function of the isolation doping (sum of spray and stop). [39]

easily destroy the front-end chips in a DC coupled sensor or the dielectric in the AC case. Precautions must be taken in the form of PTP.

In a beam splash the PTP operates to short circuit the bias resistor and drain the charge directly from the strip implant into the bias rail, protecting the coupling capacitor. The methodology is to use a small gap between the strip implant and the bias ring structure. The PTP performance is evaluated on three aspects. The onset voltage at which the short circuit is switched on should be low. A sharp decrease in resistance should follow saturating at a very high level by the dielectric damaging $100 \mathrm{~V}$.

The historical method of a simple gap has been improved upon for the ITk Strip detector. Advances were made in both iterations with PTP variants. In the ATLAS07 minis, aluminum electrodes mated to the bias rail implant were extended over p-stop channels and dropped into p-stop wrapped implants to reduce the gap (see Figures 4.18 and 4.19). These had mixed results, most performing well but with the design X1Z4 causing systematic microdischarge that required a reworking (see Section 4.3.3).

A small batch of test sensors developed upon the Z4C concept introducing a totally different design with overhanging structure, as seen in Figure 4.18 [10,40]. This socalled "gated" PTP performed the best under irradiation and was improved upon with another round of TCAD simulation before being implemented in the ATLAS12 full size sensors $[10,38]$. The "full-gate" structure suggested by simulation was compared against others in the A12 minis, as seen in Figure 4.18, and held out as seen in Figure 4.20. The fundamental difference between the gated and previous extended version are shown in Figure 4.19. It should be noted that in this figure the new PTP structure 


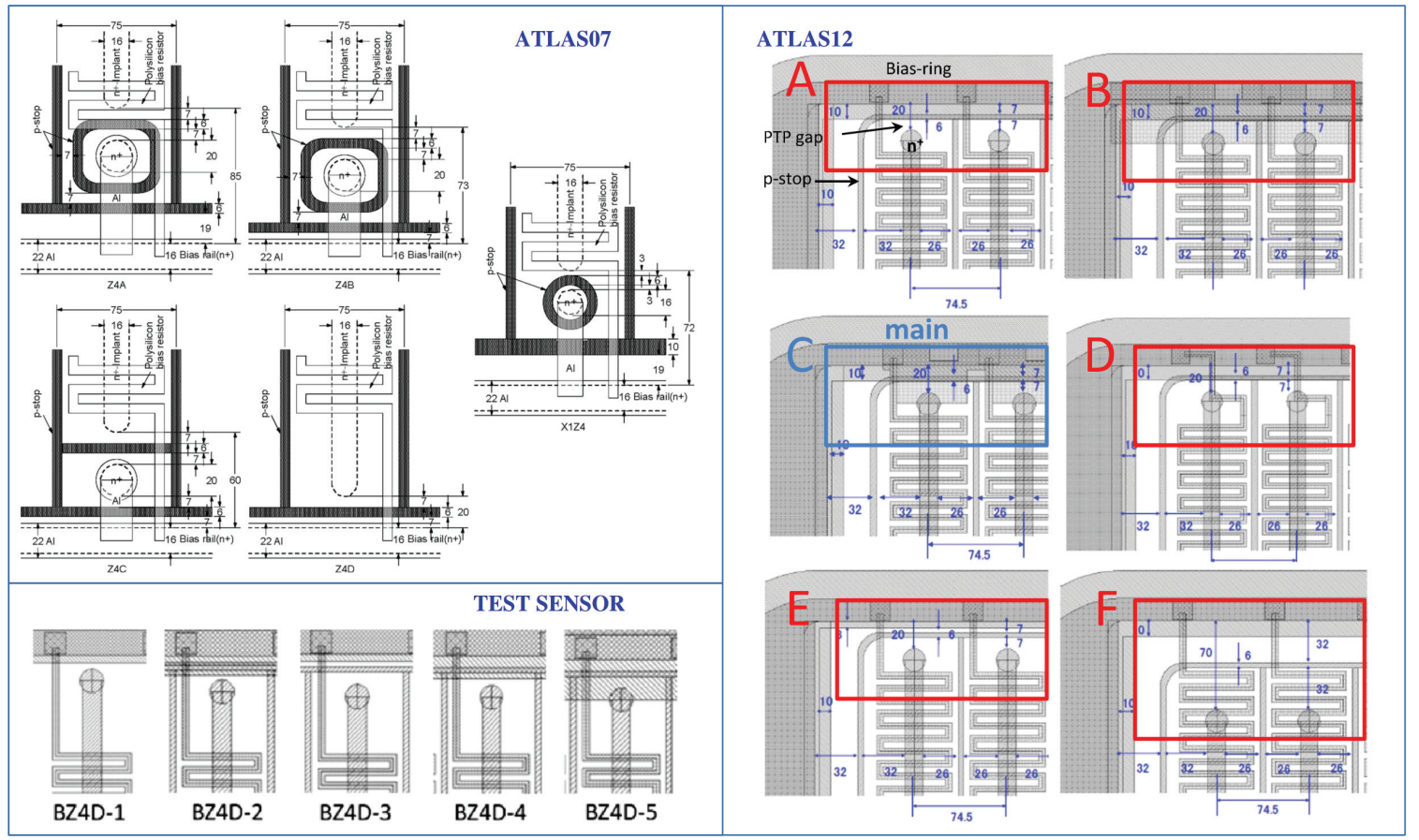

Figure 4.18: Punch through protection development for the HL-LHC. Top left; the ATLAS07 variants [29]. Bottom left; the small size test sensors PTP variants [40]. Right; the ATLAS12 variants [24].

is simulated as irradiated to $1 \times 10^{15} n_{\text {eqv }} \mathrm{cm}^{-2}$ and yet still shows field strengths one third that of silicon's avalanche value.

The punch through protection variants of the ATLAS12M minis were tested after $1 \times 10^{15} n_{\text {eqv }} \mathrm{cm}^{-2}$ of proton irradiation [24]. The results, seen in Figure 4.20 strongly supported the full-gate. The feature was considered finalized and is implemented in the A12EC and A17LS.

\section{Edge Structure}

The hermeticity and hit multiplicity requirements of tracking as well as the space considerations inherent in the inner part of the ATLAS detector motivate the minimization of dead area. In the ITk Strip detector this is obtained at the sensor level via two methods; the stereo annulus geometry (discussed in Section 4.3.3) and the use of a slim edge-width. Importantly for operation, the edge area is another more sensitive aspect of the detector combining the high potential difference of the bulk 

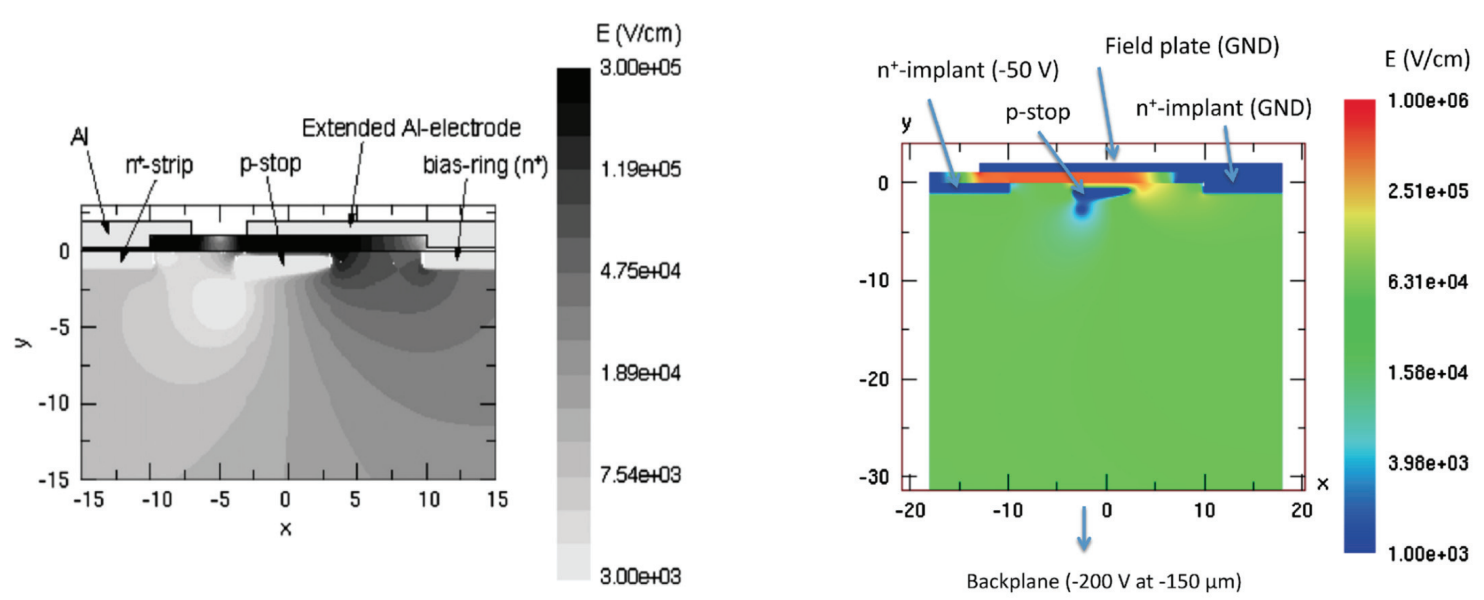

Figure 4.19: TCAD simulated comparison of advancing radiation hardness in PTP design. Left; extended electrode implemented in the ATLAS07 Z4D, unirradiated [29]. Right; the full gate structure implemented in ATLAS12 full size, irradiated to $1 \times 10^{15} n_{e q v} \mathrm{~cm}^{-2}$. Strip implant at $-50 \mathrm{~V}$ for both, refer to Figure 4.18 for structures. [38]

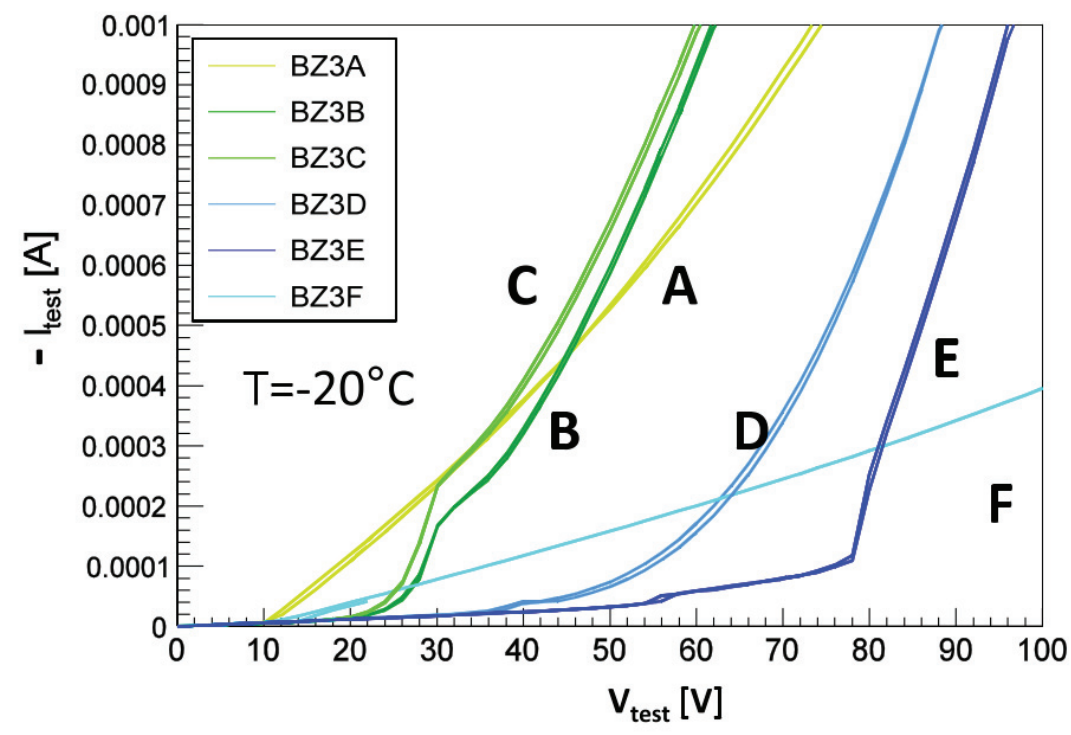

Figure 4.20: Radiation hard PTP structures of the ATLAS12M sensor submission. Current between rail and implant as a function of voltage for the six designs seen in Figure 4.18. A proton irradiated sample with a fluence of $1 \times 10^{15} n_{e q v} \mathrm{~cm}^{-2}$ is tested at $-20^{\circ} \mathrm{C}$ and $1000 \mathrm{~V}$. [24] 
with the complex charged and mobile nature of the surface.

In the ITk microstrip sensor design the sensor edges are treated with silicon dioxide and passivation layers. The associated charged interface located at the silicon bulk edge causes it to act like a self-conductive FOXFET (field-oxide field-effect transistor) and the voltage at the backplane is conducted to the edge of the top surface. The ITk design uses one floating $n^{+}$guard ring implant and a $p^{+}$edge implant. The $p^{+}$ implant extends from the edge to the CVD aluminum edge-metal structure which has the field controlling overhang into the $n^{+}-p^{+}$edge-gap.

The minimum edge-width to hold the high voltage was determined experimentally as was the optimum number of guard rings. This was accomplished with the batches of irradiated diode test sensors that bridged the A07 and A12 iterations [40,41]. The layout of the one sensor variant is shown in Figure 4.21. Edge cuts ranging from $80 \mu \mathrm{m}$ to $1011 \mu \mathrm{m}$ were tested with both $\mathrm{p}$ and $\mathrm{n}$ type edge implants. A variety of narrow, medium, wide, and one, two, or three guard rings (as shown on the left side of Figure 4.22) were implemented systematically in the range of edge widths. The sensors were irradiated with $70 \mathrm{MeV}$ protons up to a fluence of $1.2 \times 10^{16} n_{\text {eqv }} \mathrm{cm}^{-2}$ and annealed $80 \mathrm{~min}$ at $60^{\circ} \mathrm{C}$.

The edge width results were quite definitive in the diodes and are summarized in Figure 4.23. Before irradiation the p-type diodes exhibited the ability to hold $1000 \mathrm{~V}$ if the edge width was larger than $400 \mu m$ (left). From the IV curves (centre), the plots with edge width of $\simeq 400 \mu \mathrm{m}$ show one guard ring is slightly preferred. Finally on the right side of Figures 4.22 and 4.23, the evolution of microdischarge with voltage and fluence for the different structures show that these trends were still observed after irradiation.

The diode tests approved the implementation of two dicing streets in the ATLAS12 series. This allows direct comparison of the conventional $\simeq 910 \mu \mathrm{m}$ edge width against that of a slim design. $450 \mu \mathrm{m}$ was chosen as the specified slim edge-width simultaneously setting restrictions on the dicing quality. Figure 4.24 shows an ATLAS12 sensor with the conventional cut [42]. The slim dicing street is shown in yellow. The dual dicing streets in the full size sensor allow a realistic comparison between the two. IV curve results are shown before (left) and after (right) slim dicing in Figure 4.25. Different trends are exhibited for the stereo (A12M) and axial (A12A) designs. 


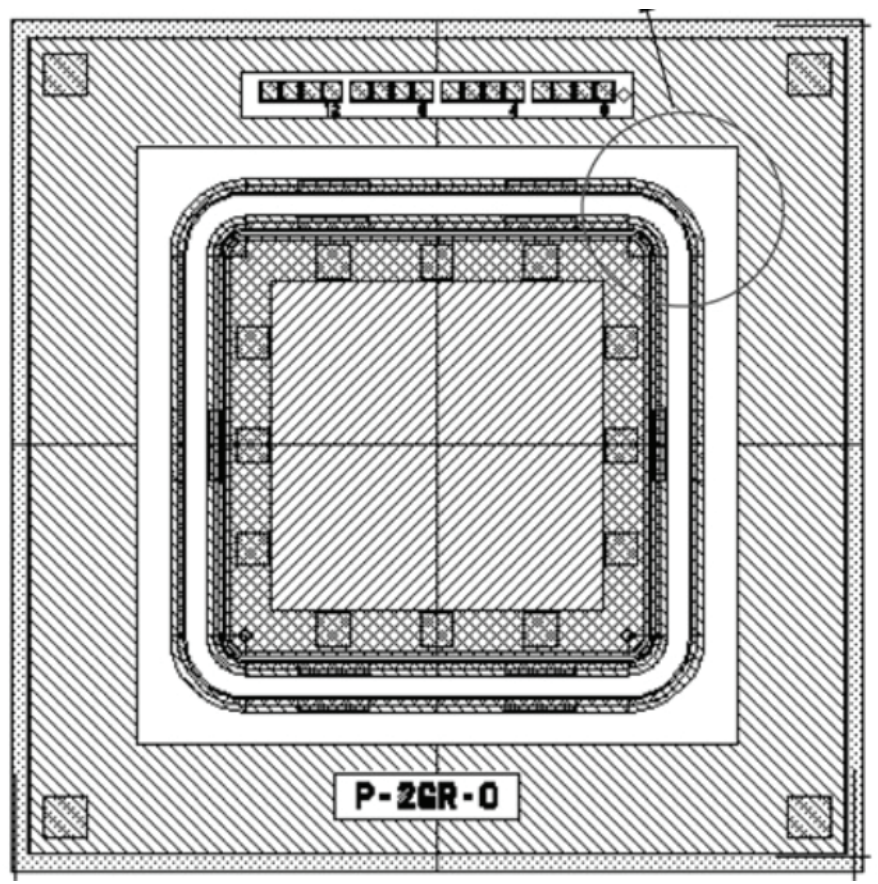

Figure 4.21: An example layout of the edge structure test diodes. The circle corresponds to the focused view offered by Figure 4.22 left. Outer dimension of the diode is $4 \mathrm{~mm}$.

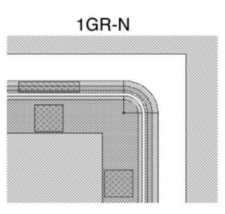

2GR-M

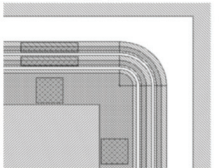

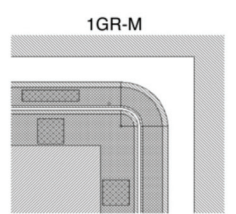

2GR-W

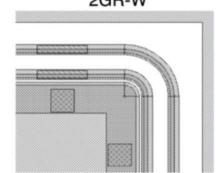

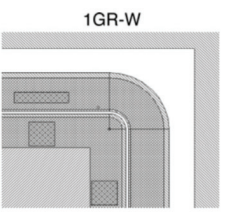

3GR-W

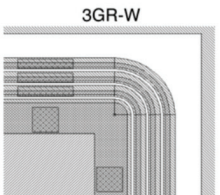

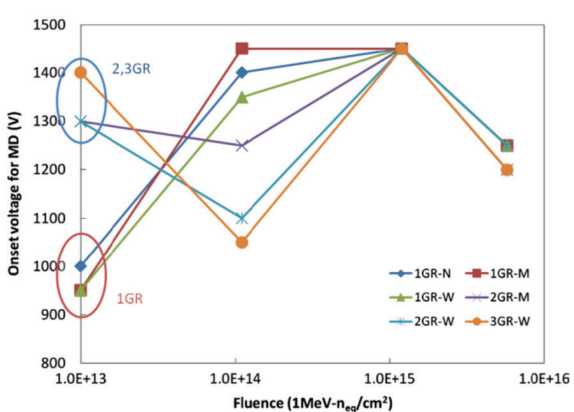

Figure 4.22: Guard ring structures tested in the diode test sensors. Left; structure designs denoted by ring multiplicity and feature width [41]. Right; microdischarge onset voltage for the different structures as a function of fluence $(70 \mathrm{MeV}$ protons) [40]. 

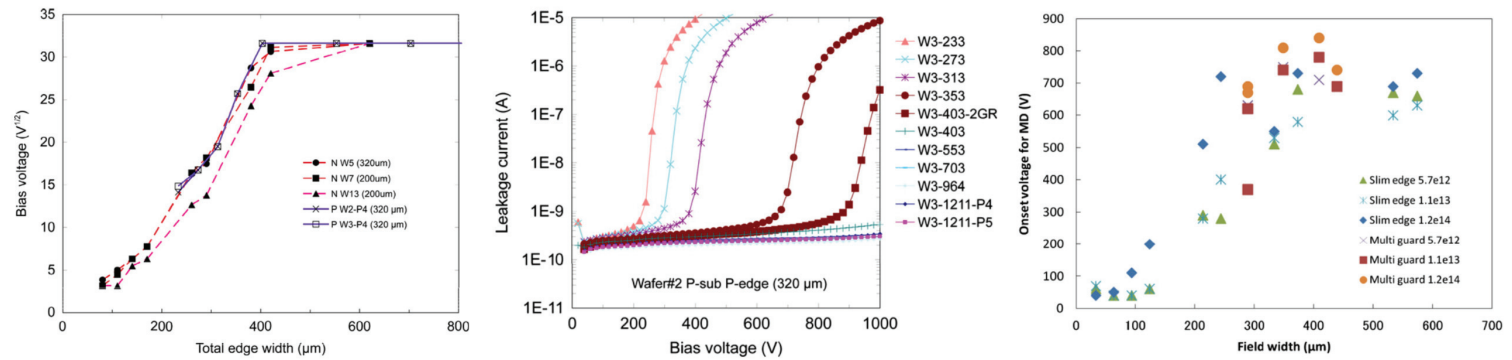

Figure 4.23: Results of edge width tests conducted on the diode test sensor batch. Left; microdischarge onset voltage as a function of edge width for $\mathrm{p}$ and $\mathrm{n}$ type sensors, the saturation point is $1000 \mathrm{~V}$. Centre; IV curves for variant edge width, from $233 \mu \mathrm{m}$ to $1211 \mu \mathrm{m}$ (legend: wafer- total edge width in micron). One guard ring is preferred from the W3-403 and W3-403-2GR data [41]. Right; onset voltages for microdischarge (MD) as a function of edge width and guard structure for irradiated samples. [40]

The success of the slim edge thus far has seen it implemented as the only option in the restricted geometry of the A12EC [30]. An increased edge width of $500 \mu \mathrm{m}$ is used along the straight edges only to allow the desired bulk structure contact width. The long strip barrel prototype will reserve the right to move back to the two edge option but with the slim-edge as a baseline [31].

At the current stage the very recent release of the first sensor with the all-new end-cap geometry will push this design further than ever before and thorough testing will be required. This testing is happening at Carleton University and other institutes across the globe. It is the subject of Sections 5.3 and 5.5 and one of the main topics of this thesis.

\subsubsection{End-cap Specific Considerations}

The circular nature of the end-cap is a necessary inconvenience to the production and tracking in a full coverage detector. Hermeticity must be obtained and the tracking elements must be optimally oriented radially outward from the beam line. The conventional coping method in the high energy physics field is the trapezoidal wedge shaped detector which is rotated akin to the barrel sensors to obtain the stereo angle [64,68]. In other words the sensors are placed rotated on the local supports. The ITk's stereo annulus design, an improved divergence from this tradition, has been 


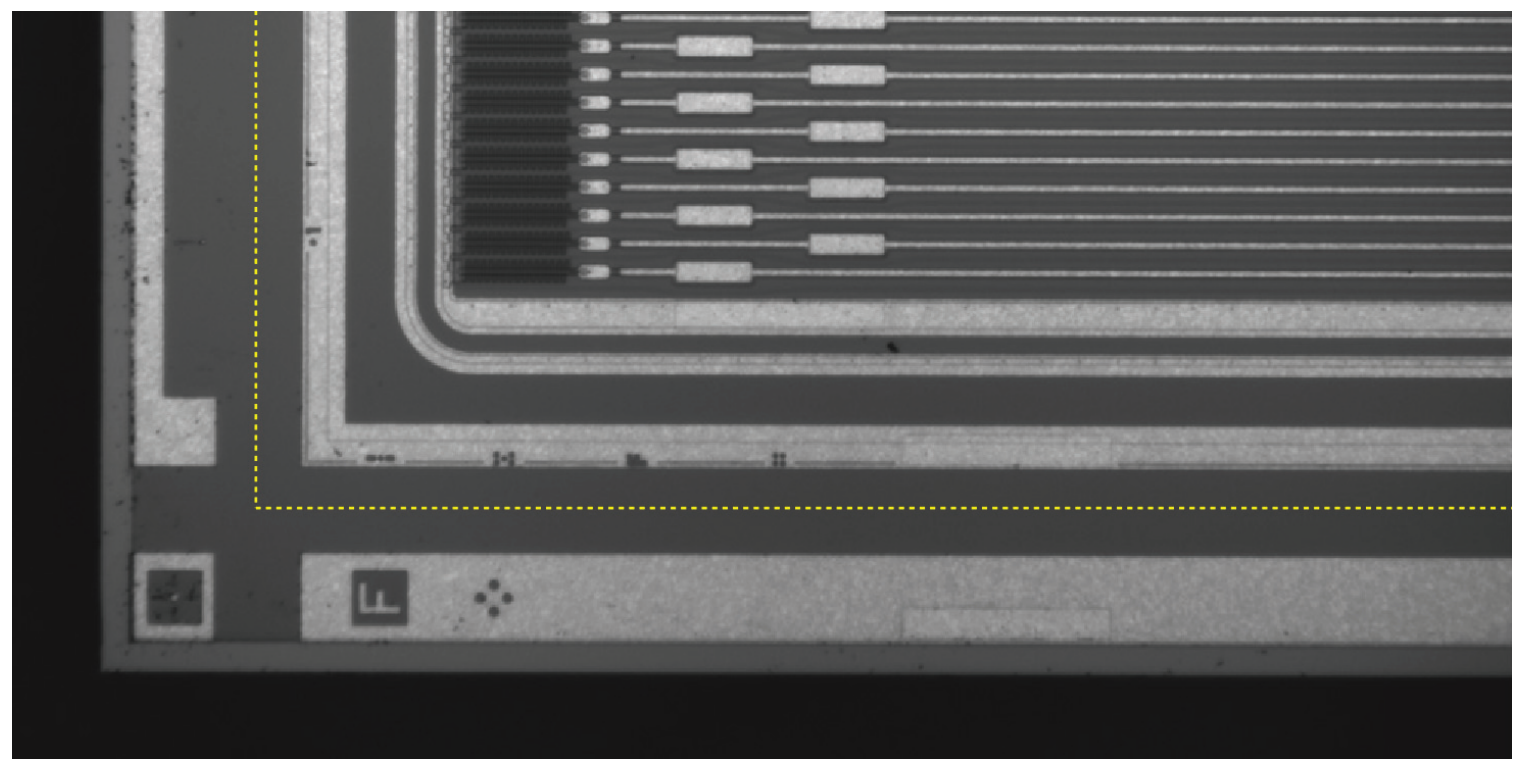

Figure 4.24: Slim edge implementation. Photograph of the corner of an ATLAS12 sensor surface with conventional cut. The slim edge dicing street is shown in yellow. [42]
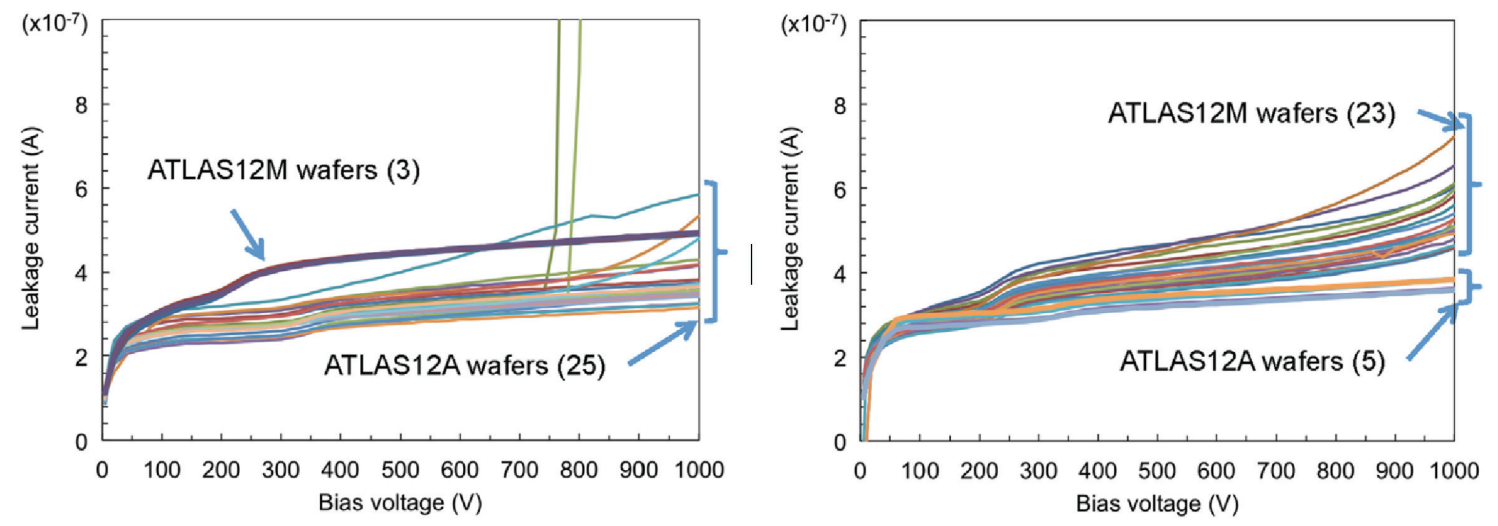

Figure 4.25: Slim edge dicing effect on the IV curve of the ATLAS12M and ATLAS12A sensors. Left; sensors before slim dicing (sensors with conventional edge of $\simeq 910 \mu m)$. Right; sensors after slim edge cut $(\simeq 450 \mu m)$. [42] 
described in Section 4.1.2. It optimizes the layout of the end-cap microstrip detectors in two ways; stereo strips integrated right into the silicon allowing a reduction in dead space, and arced edges allowing the strips of each row to be the same length. These advancements were made in two stages, outlined below, the second of which is currently still under test (Chapter 5).

\section{Wedge Shaped Stereo Sensors}

The ITk collaboration has developed several designs of wedge shaped stereo sensor in different prototypes. The wedge shape is well entrenched, cost-effective and offers the simplest interlocking for reasonable detector coverage. However, in its most conventional configuration (symmetric) the wedge is incompatible with the stereo strips. This results in the "loss" of strips in the corners of the wedge (due to readout chip bonding proximity). Even when a stereo angle is integrated into the wedge shape the issue of unequal strip lengths remains affecting the capacitative response of the channels.

Several attempts to address the "lost" or "orphaned" corner strips were tested in both the ATLAS12 mini- sensors and the petalat project [10,32,43,83,84]. Two examples of A12 configuration, seen in Figure 4.26 [83], display the basic distinctions. The DC "ganging" technique (left) connects the orphaned strip metal to a neighbouring implant. The AC ganged strips (right) are connected to a neighbouring strip metal. Both methods were tested with different neighbour configurations [32,83,84]. The A12 $(1 \mathrm{~cm})^{2}$ minis were also irradiated and showed minimal charge loss but increased interstrip capacitance for the ganged strips.

Another innovation of the ITk Strip project was the use of a second metal layer, oxide separated, above the strips. In this respect bond pads of the almost radial strips would be adapted to the tangentially arranged readout chip bond pads. This allows the FE wirebonds to be shortened and straightened improving production yield $[32,43]$. Extensively tested the so-called "embedded pitch adapter" variants are shown in Figure 4.27 [43]. Interestingly, a decrease in interstrip capacitance and increase in "bulk" capacitance is observed. This module has been taken to beam tests at the Diamond Light Source synchrotron and the $5 \mathrm{GeV}$ particle beam at DESY (see Section 2.7.3).

Testing of composite sensor structures (like those planned for the outer rings of 

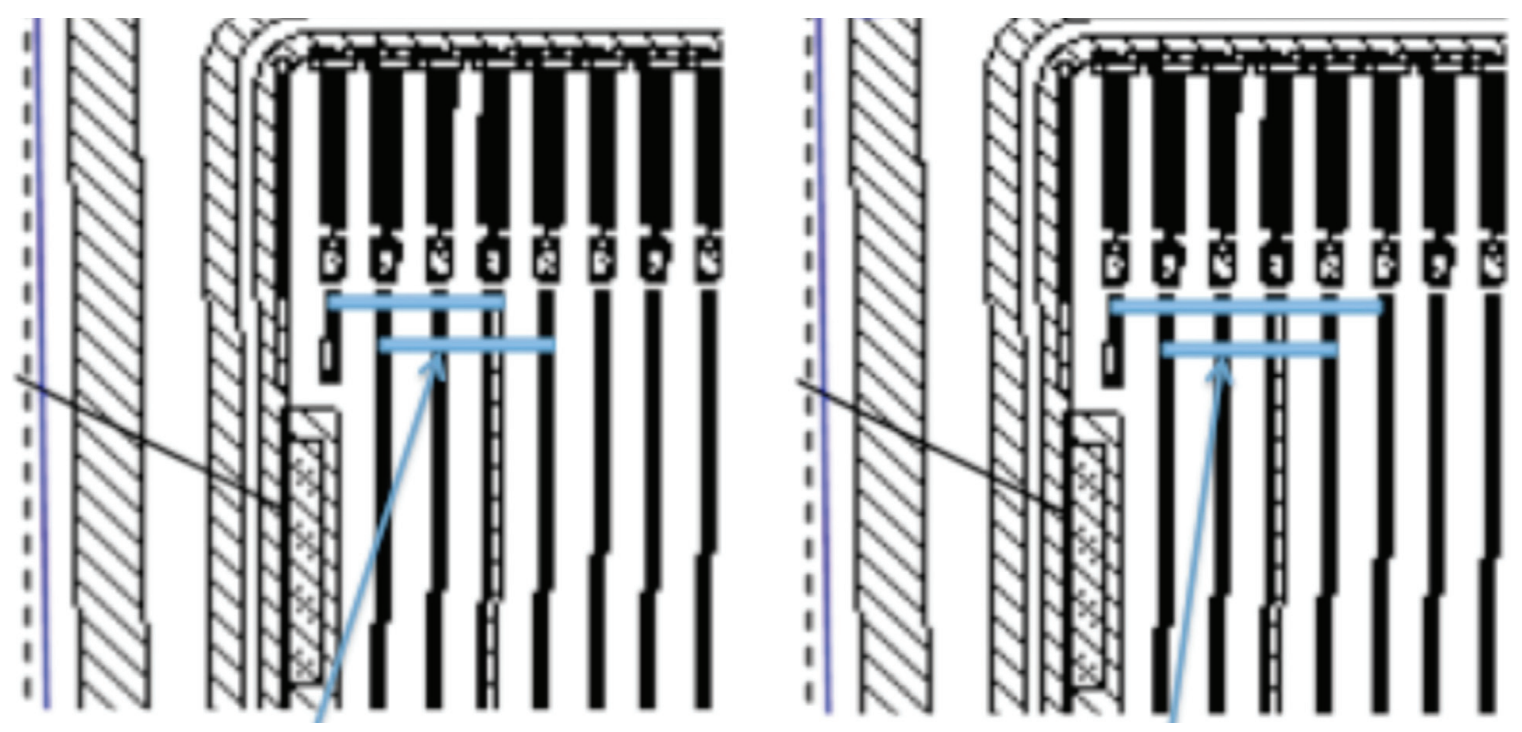

Figure 4.26: Examples of ganging schemes implemented in the ATLAS12 $1 \mathrm{~cm} \times 1 \mathrm{~cm}$ sensors. Light blue denotes the interstrip connections. Left; DC ganging. Right; AC ganging. [41]

the ITk Strip) was also implemented in the petalet program as seen in Figure 4.6

\section{Stereo Strips in Large Areas}

The move to the $\sim 97 \mathrm{~mm} \times 97 \mathrm{~mm}$ large area of the ATLAS07 ITk barrel sensor prototype was accompanied by two issues in the mask that were related to the size increase. The first issue was related to the PTP structure X1Z4 (see Figure 4.18) while the second was related to the brand new implementation of stereo strips. In both cases systematic microdischarge was observed via its associated IR emission. This is seen for the stereo strip case in Figure 4.28. The issues were understood using TCAD simulation. For the PTP case they were associated with the action of multiple narrow p-stop arrays acting like larger conglomerates, the microdischarge location can be inferred from the left side of Figure 4.19.

The localized IR signature of the microdischarge when appearing systematically can illuminate shortcomings in the design. Figure 4.28 shows the stereo strip related mask issue. In the left photo taken with hot-electron equipment [29] is the IR emission of microdischarge, its systematic pattern indicative of a design flaw. An example of the type of TCAD informed mask improvement along with the problematic version 
(a)

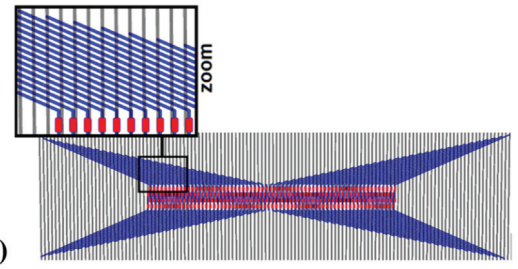

(c)

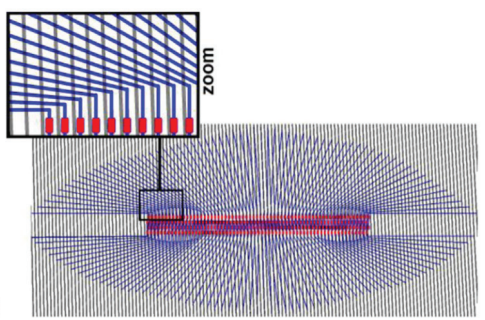

(b)

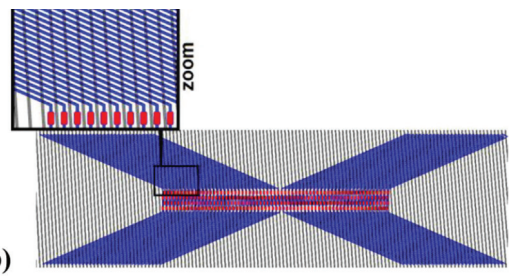

(d)

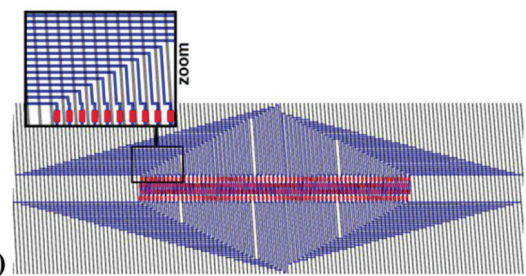

(e)

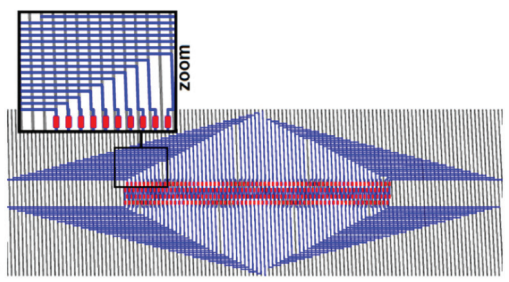

Figure 4.27: Embedded pitch adapter variants studied in the 2016 testbeam data. [43]
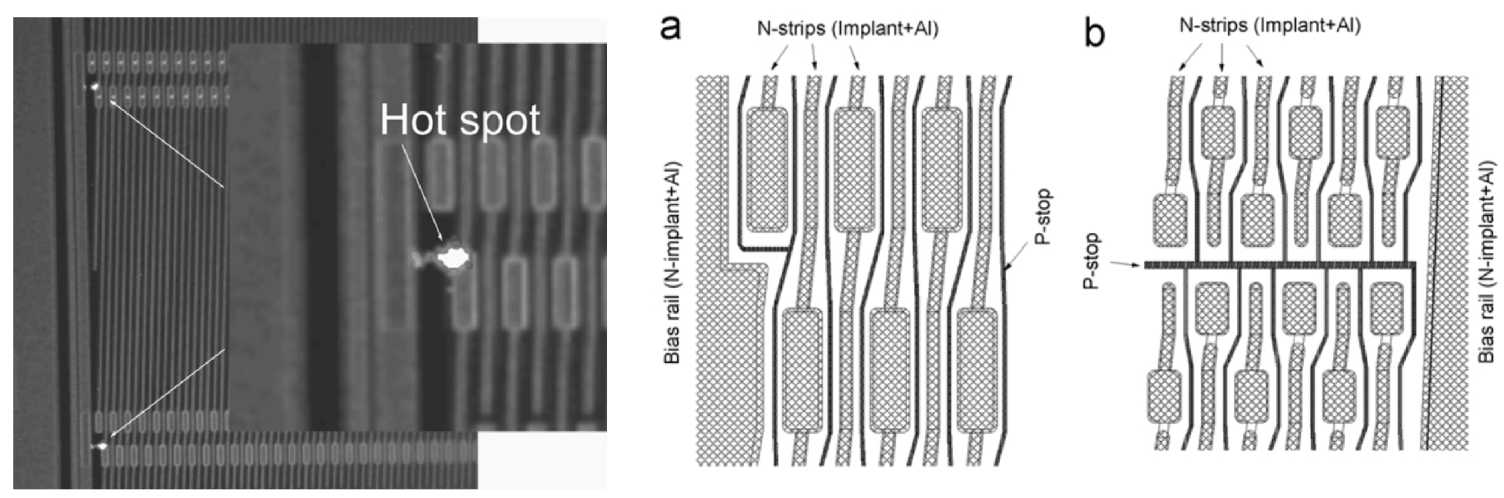

Figure 4.28: Microdischarge and TCAD assisted large area, stereo strip, sensor mask refinement. Left; hot-electron image of the systematic microdischarge. Centre; the mask feature causing the problem. Right; the mask fixes successfully implemented. [29] 
are shown in the right and centre portions of Figure 4.28 respectively. The stereo strip fixes were implemented along with a removal of the poor PTP structure in a well performing second batch of ATLAS07 [29,45]. Subsequently, the A12M and so far, the A12EC, have seen successful stereo strip implementations.

As has been noted that the reversible and reproducible nature of the microdischarge coupled with its related IR emission and burst noise increase make it a prime tool for investigation into more isolated defects in the sensor due to processing [55] (see Section 5.4.1).

\section{Stereo Annulus}

The name follows naturally for this design which is introduced in Section 4.1.2 and seen in Figure 4.2. The disadvantage of orphaned strips is totally negated in the use of a stereo angle skew in the actual sensor shape. Equal strip length is also incorporated when the design is extended to include the annulus feature on its tangential sides. This allows all the radial stereo strips in each row of each sensor to be of the same length with important consequences for the channel response and noise characteristics of the module [1]. As well, benefits to the quality testing program as outlined in Section 5.1 are also offered by greater electrical uniformity. However, this advantage comes at the cost of reduced compatibility between the sensors in the radial direction (ie. on a petal) and a small increase in dead area compared to a stereo wedge. Other concerns such as the bonding to the ABCStar are accommodated into the careful designs which maximize the six inch wafer process across the petal.

The ATLAS12EC prototype sensor is the first of its kind and will allow a good test of the design before it is implemented in the six geometries of the ITk Strip end-cap. Several features will be of special interest as dictated by the first ever fabrication of this unique shape. The circular edge cuts are a rarity in the microelectronics industry and experience will have to be developed at the sensor source for this more complicated cut (approximated by up to 16 flat sections which are matched by ring [30]). This necessitates extensive testing considering the sensitive nature of the edge structure already amplified by a slim-edge width and high ionizing doses. Also of interest will be the interstrip measurements due to the relatively large variance and angular nature of the pitch across the stereo annulus surface. 


\section{Chapter 5}

\section{Sensor Testing at Carleton University}

In this chapter the discussion of the ITk Strip sensor comes to a natural conclusion with a presentation of the current state of development and evaluation. In particular, the focus is on work that has been done at Carleton University to build a production ready test site and the results that have been obtained so far. This is the main topic of this Master's thesis and constitutes my work on the ITk Strip sensors.

Development of an able silicon microstrip sensor test facility is not a trivial thing. The Carleton University site will see roughly 5000 sensors pass through its cleanroom doors before the end of 2023. Historically, at Carleton University the ATLAS experimental group has been focused on gas phase detector fabrication and testing. Thus, one year prior to the writing of this document the extent of knowledge and equipment for the sensor testing portion of the site was quite minimal. Now, in this document, test results of more than sixteen ITk sensor prototypes are shown (Sections 5.3 and 5.5). These include some of the first results obtained for the stereo annulus sensor design a major milestone for the endcap assembly. The results are preceded by discussions on the testing program that will be implemented at Carleton (Section 5.1) and the development of the test facility (Sections 5.2, 5.3, and 5.4). Ultimately, successful operation of the test site will require an intimate knowledge of both equipment and the sensor under scrutiny. The careful development in the last two chapters of the theoretical and experimental background of these sensors gives the required context not only for the test results, choice of instruments, and techniques presented in this chapter but for the years to come. 


\subsection{ITk Strip Sensor Testing Procedures}

The ITk is unique with an enormous silicon surface coverage, daunting component density, and enduring experimental constraints. These require of the ITk project a well developed production model and thorough testing regime at both the prototyping and production phases. Luckily, the collaboration can build on the experience and successes of the production, installation, and operation of the ATLAS SemiConductor (SCT) detector. However, in the ITk with an increase in number of silicon microstrip modules by $388 \%$ (17888 vs. the SCT's 4088) innovation, in the form of prototype $\mathrm{R} \& \mathrm{D}$, is an absolute must.

These sensors, like all the on-detector components, must operate with high reliability and essentially no intervention for the ten plus years of operation. A robust quality control and analysis structure is needed to try and maintain the full functionality of the detector for the experimental duration. The content of these tests and the related specifications are developed over the course of the intense $R \& D$ effort, the current iteration of the test programme is presented below. This is the state at the time of the ITk Strip Technical Design Report [1]. It, and the technical specifications for the ITk prototypes $[30,31,67,81]$ serve as the main source for the tests outlined here with minor contribution from developed technique.

It should be noted that quality control (QC) is the process which ensures that the production components all match specification. It consists of tests conducted on every single production item. Quality assurance (QA), on the other hand, is focused on optimization and reliability of the design and the manufacture process, it takes place both during the R\&D phase as well as at a small scale during production (for more complete consistency monitoring).

\subsubsection{Sensor Quality Control}

Sensor quality control is aimed at ensuring that all sensors entering the ITk are fully functional. It takes place during the production phase and is conducted on all 17888 sensors (plus spares). Functionality is interpreted in terms of the specifications which are given by the experimental requirements, feasibility considerations, and honed over iterations of R\&D. Table 4.1 summarizes the sensor specifications including some properties that are obtained through the QA tests.

The QC program involves two classes of non-destructive tests; a set of fundamental 
tests conducted on every sensor, and a more involved test regime conducted on a sensor subset. Time and infrastructure considerations as well as estimates on the failure rates for different aspects of the sensor design are the factors that drive the size of the subset undergoing extended testing. The initial expectations are that $10 \%$ of each manufacturer batch will be subject. How the sensors respond to these tests over the earlier period of the production run will dictate the size of the subset towards the end.

\section{General Testing Considerations}

As a large area solid state silicon device the ITk sensor is extremely sensitive, both mechanically and electrically. Cleanroom conditions (ISO6) are expected, and are motivated by the need to wire-bond later during assembly. The sensors have a delicate response to temperature and humidity conditions. This necessitates regulation of the environment to reasonable or specified levels with the risk of biasing test results. With the photo-sensitivity of the depleted silicon, electrical tests must be conducted in light tight enclosures. At $300 \mu m$ the sensors are thin, coupled with their large area, they are extremely fragile and demand special handling techniques. In the operation of the ITk the maximal high voltage foreseen for the Strip detector is $700 \mathrm{~V}$, this is reflected in the test specifics detailed below.

Connection to the bulk of the silicon is obtained via the backplane (or equivalent front-side edge-metal contact pad) and the bias rail contact pad. Reverse bias (used interchangeably with 'bias') is obtained by sourcing a negative voltage to the sensor backplane. It is useful to remember that the bias rail will be maintained as the electrical ground on-sensor (unless otherwise specified). Electrical joining to the sensor itself can be achieved by use of probestation micromanipulator controlled needles, or via wire-bonding in a custom test frame or jig.

\section{QC Tests Conducted on All Sensors}

Four fundamental measurements are conducted on every single one of the 17888 plus sensors possibly entering the ITk; two mechanical and two electrical tests of the sensor bulk. The two mechanical tests are visual inspection and basic sensor metrology (sensor bow). The sensor electrical character tests are leakage current as a function of reverse bias and full depletion voltage determination. 


\section{Visual Inspection:}

In this test the general mechanical state of the sensor is evaluated to identify any sensors which may be more susceptible to the stresses of their expected life in ATLAS. The surface of the sensor is inspected manually under magnification. A higher magnification survey is focused on the most sensitive region in terms of processing, that of the edge. The sensor surface is expected to be uniform and free of significant scratches with the edge cut clean and devoid of chips or cracks. A high resolution, deep-colour profile, digital image of the full sensor surface will be captured for posterity.

\section{Basic Metrology:}

This test involves measuring the sensor flatness or bow. This is an important quantity to ensure assembly reliability at larger scales of the detector (gluing issues emerge with large bowing), reliability during operation (due to deformations during thermal cycling), and to meet the space requirements for the detector. A non-contact $\mathrm{x}, \mathrm{y}, \mathrm{z}$ measurement of the surface is made in a grid of roughly one centimetre spacing (100-130 points per sensor). The sensor must be un-stressed (under gravity) during measurement. The sensor bow (difference between the highest point measured onsensor and the lowest point) must be less than $200 \mu \mathrm{m}$ with a single point measurement accuracy on the order of one micron.

\section{Leakage Current : IV Curve:}

The IV curve is a basic test of the sensor's bulk electrical quality and allows an investigation of susceptibility to microdischarge. A source measurement unit (SMU) with high voltage capability and picoamp precision is used to measure the leakage current flow through the sensor bulk as a function of reverse bias voltage. The test is conducted beyond the maximal operating voltage (to $750 \mathrm{~V}$ ). Compliance limiting is required and the test is stopped if microdischarge is observed. Temperature is also recorded during the test allowing for normalization. The $20^{\circ} \mathrm{C}$ normalized, guard ring inclusive leakage current is expected to be less than $0.1 \mu \mathrm{Acm}^{-2}$ at $700 \mathrm{~V}$. There shall be no observation of the sharp current increase of microdischarge; defined as $\frac{(d V / d I)}{\left.(d V / d I)_{\min }\right)}=5$.

\section{Full Depletion Voltage : CV Curve:}

In this test the full depletion voltage is inferred from the inverse square relation 
between depletion depth and capacitanc Equation 3.4. As discussed, the full depletion voltage, $V_{f d}$, has important implications for charge collection especially in sensors planned for a high radiation dose. Two instruments are required; a compliance limited DC SMU for sensor bias and a nanofarad resolution CV meter (AC voltage stimulus and $\mathrm{RC}$ measurement). With the $\mathrm{AC}$ source connected to the backplane and the probe connection at the bias rail the bulk capacitance is measured in series with the array of bias resistors. Data is taken as the bias voltage is increased up to maximal and a plot of the inverse square of the capacitance as a function of voltage is made. The full depletion voltage is extracted from the intersection of the resistor-like linear rise and saturation plateau (see Figure 3.7).

\section{QC Tests Conducted on a Subset of Sensors}

For each manufacturer batch a subset of sensors are subject to an extended testing regime focused on ensuring the full reliability of the numerous technical aspects of the design. With batch fraction sizes informed by studies of feature failure rates and tunable over the course of production this scheme allows for high efficiency and high reliability. The extended regime consists of two phases. In the first phase the sensors are subject to a long term leakage current stability test as well as a full functionality test of every single strip on the sensor. In the second phase, a set of strips distributed across all segments on the sensor surface is chosen. Further investigation of the surface processing is performed on this strip subset. This includes detailed measurements of inter-strip resistance and capacitance, the strip, implant, and poly-silicon resistor resistance. Quantification of the punch through protection structure performance is also completed. It is conventional to repeat the IV and CV curves after the extended testing is complete.

\section{Advanced Metrology:}

A further examinations of the basic mechanical properties of the sensor used to monitor batch by batch consistency. In this test the thickness of the sensor and its uniformity are measured with sub-micron resolution. The thickness expected is $(310 \pm 10) \mu m$.

Leakage Current Stability:

In this test the bulk leakage current at a constant bias of $700 \mathrm{~V}$ is monitored for 
variations over a 24 hour period. This test serves primarily as an examination of the surface and edge processing of the batch. A compliance limited, high voltage capable SMU is used, as is dry nitrogen atmosphere $(<5 \%)$ and room temperature $\left(20^{\circ} \mathrm{C}\right)$ conditions. Logistically, it is foreseen that this test will be conducted on several sensors in parallel.

Striptest:

This is a thorough test of the basic properties of the full array of microstrips on the sensor surface. It is a probe test in which every single functional strip is tested. Issues in the conductivity of strip or implant and in the uniformity or permittivity of the dielectric layer are identified and the bias resistor resistance and dielectric coupling capacitance are quantified.

The circuitry for this test is more complicated and is shown in Figure 5.1. A compliance limited SMU is used to isolate the strip structures via partial depletion of the sensor bulk. To test the strips themselves requires a picoamp resolution SMU, a CV meter, and a switchable circuit. The low end of strip test circuit (voltage ground and CV probe) is connected to the bias rail (ie. a common ground is used on the low end of the switchable circuit and the bias circuit). The high end connection is initiated via probe needle contact with the $\mathrm{AC}$ pad of the first strip on the sensor surface. The picoamp test SMU is routed through a current limiting resistor.

Electrical reliability of the strip, implant and dielectric are examined by sourcing of test voltages of reasonable strength (ten and then one hundred volts). The strip is shorted to ground for 500ms to deplete residual charge buildup prior to switching in of the $\mathrm{CV}$ meter. A small AC voltage is applied to the strip metal and with the CV meter configured for a series measurement the bias resistance and coupling capacitance are extracted. The strip contact is broken and initiated at the next strip on the sensor and the process is repeated. The number of strips with significant deviation from the specifications (Table 4.1) is expected to be less than one percent and uncorrelated (in a topological sense) sensor-wide. The bias resistance and coupling capacitance are expected to be $(1.5 \pm 0.5) M \Omega$ and greater than $20 \mathrm{pFm}^{-1}$ at $1 \mathrm{kHz}$, respectively.

The sheer multiplicity of strips to be probed per sensor and strict time requirements of the production model necessitate the automation of this test. The use of switching matrix, semi-automatic probestation, custom multi-needle probe cards, and largely automated testing procedures is foreseen. 


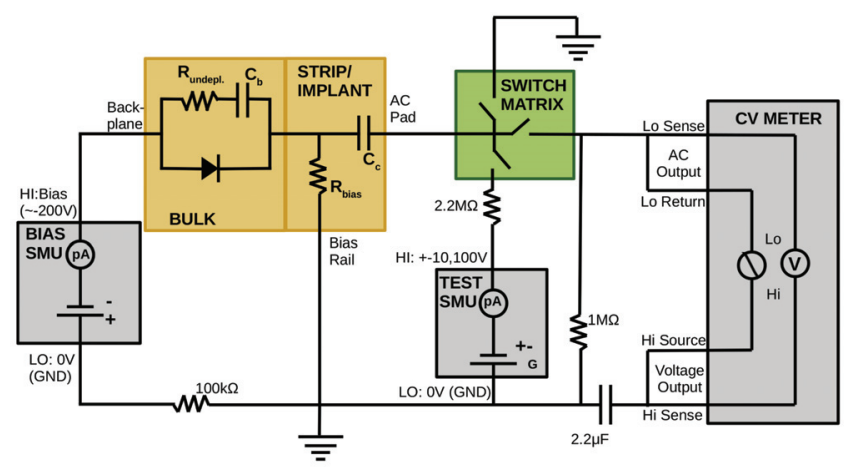

Figure 5.1: Equivalent circuitry for the "full striptest" conducted on a subset of the ITk sensors.

\section{Interstrip Capacitance:}

The tests examining the electrical properties between strips are strong investigations of the surface and mask processing quality. In this test the capacitance between neighbouring strip structures is measured with important consequences for noise and channel cross-talk in the finalized module. It is a probe test conducted on a representative sample of the strips on the sensor. The interstrip capacitance is determined as a function of bias voltage. As seen in the left side of Figure 5.2, three probe needles are used to contact the AC pads of three neighbouring strips. The central strip is connected to the $\mathrm{AC}$ voltage source of a sub-picofarad resolution CV meter with the neighbouring needles connected to the return side. A compliance limited SMU, connected to the sensor bulk raises the bias voltage from $0 \mathrm{~V}$ to $300 \mathrm{~V}$ while the capacitance is measured with the CV meter in parallel configuration. The expectation is that the interstrip capacitance is less than $1 p \mathrm{Fcm}^{-1}$ at $300 \mathrm{~V}$.

\section{Interstrip and Bias Resistance:}

In this test the bias resistor and resistance between neighbouring strips is measured as a function of bias voltage. The bias resistor is an important feature dictating the level of the strip's response to a charge liberated in the bulk. This test allows for an analysis of this feature beyond that of the striptest. It also allows conclusions on the strip isolation performance, an important quality especially in considerations of charge sharing and irradiation. The equivalent circuitry is seen on the right side of Figure 5.2. 

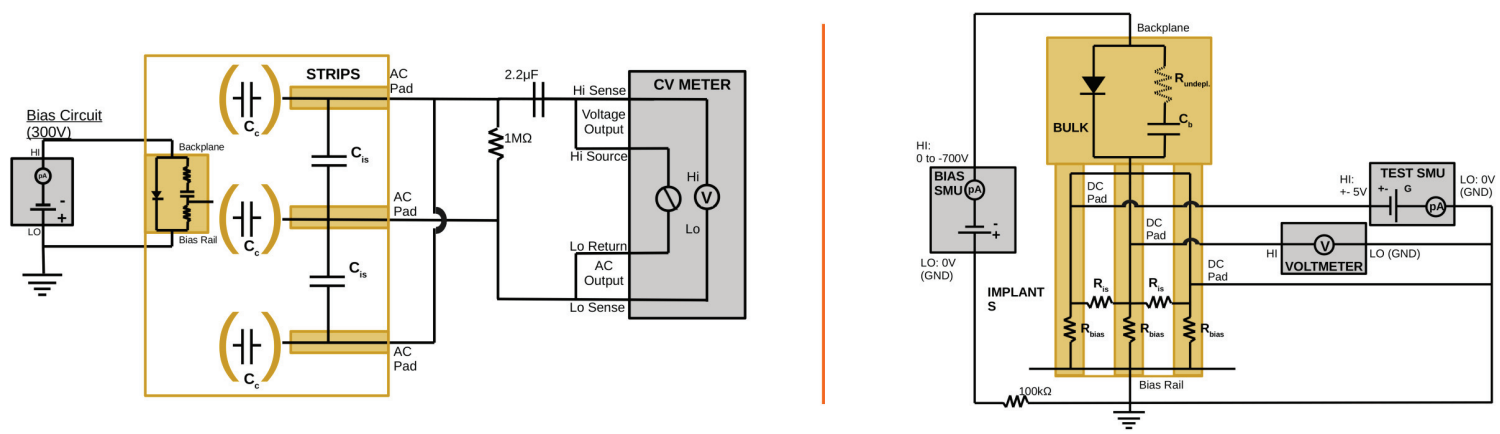

Figure 5.2: Equivalent circuitry for the interstrip tests conducted on a subset of the ITk sensors. Left; the AC interstrip capacitance measurement. Right; the DC interstrip resistance measurement.

The sensor bias is achieved using the usual DC compliance limited SMU connected to the bias rail and sensor backplane. The interstrip measurement requires two high impedance instruments; a picoamp resolution SMU and a sub-millivolt resolution voltmeter. A common ground is established at the low sides of the voltmeter, test SMU and bias SMU. Using three needles the DC pads of three neighbouring strips are contacted. The central strip is connected to the voltmeter and the neighbours are connected to the test voltage source and to the common ground respectively. At each step in a ramp of the bias voltage from $0 \mathrm{~V}$ to $700 \mathrm{~V}$, the test voltage is ramped from zero to five volts. The current flow through the parallel circuits of the bias resistor and silicon to the second neighbour strip is measured on the test SMU. The voltage induced on the central strip is likewise measured. This allows the extraction of the interstrip contribution to resistance from that of the bias resistor. The resistance values are extracted at the plateau of the profile versus bias voltage (ie. when strip isolation is achieved). The expected bias resistance is $(1.5 \pm 0.5) M \Omega$ while the interstrip resistance is to be at least one order of magnitude greater.

\section{PTP Structure:}

Tests of the punch through protection structures are measured on the strip subset to investigate the manufacturing consistency of this feature. A compliance limited test voltage is applied to the implant and ramped, the current to sensor ground (bias rail) is measured. The sensor bulk is held in a constant, partially depleted state of 300V for strip isolation. The PTP gate's onset voltage and slope are identified and the leakage current is examined as a function of the test voltage. The acceptance criteria 
for these properties has not yet been set.

Strip and Implant Resistance:

These tests examine more closely properties examined during the striptest and are conducted to monitor consistency in the fabrication of these numerous base features. They are probestation tests conducted on the representative strip subset. They are simple tests measuring the strip or implant resistance via a low voltage IV curve using a picoamp resolution SMU. For the case of the $\mathrm{n}^{+}$implant contact is made to the two DC pads at either end of the strip structure. In the aluminum strip metal case contact is effected at the corollary AC pads.

\subsubsection{Sensor QA}

The QA tests are statistical studies focused at illuminating shortcomings in the design, manufacture process, or batch manufacture that may not be symptomatic under the normal QC regime. To this end, these programs often employ "destructive" testing (induction of stresses above that expected for operation) with the component response evaluated, most often, via the QC methodologies.

With these goals QA encompasses both smaller scale batch based testing during production and the means by which the sensor design comes to its well assured fruition (a majority of the R\&D phase). During production, samples undergoing the specified QA are considered sacrificial and will not be incorporated into the ITk itself. The exceptional radiation fluences expected in the HL-LHC demand a special focus to radiation testing. The irreversibility of these tests naturally put them under the realm of QA and they are the focus of many of the studies in the R\&D phase.

\section{QA During Production}

QA tests during production are conducted on a very small set of sensors for each manufacturer batch. Since the nature of these tests is destructive, different sensors will be subject to different tests. The focus of the production QA is threefold; mechanical stress, long-term IV stability, and radiation hardness. Despite requiring the samples be discarded, these tests are necessary to ensure the full reliability of the product throughout the multi-year production run.

Long-term stability and IV curves are conducted to higher voltages (1000V) than 
during the quality control. The stability tests will be maintained for longer periods and the humidity dependency of the leakage current stability will be investigated.

The induction of mechanical stresses are examined at several levels. With the slim, $300 \mu \mathrm{m}$ nominal thickness of the sensors, micro-cracking is an issue. Susceptibility to micro-cracking during assembly is evaluated by repeated vacuum cycling of the sensor to a probestation chuck. IV curves are used for examination. The stresses induced in the sensor and larger assemblies by the gluing procedures (hybrid to sensor, sensor to local support) are also examined. A small subset of components at the various construction stages are subject to thermal cycling to induce stresses, with the sensor bulk IV performance and module noise levels yielding information on the stress response.

Radiation performance is analyzed at the batch level by conducting irradiation campaigns on the numerous minis that come in the wafer and small counts of full size sensors. The electrical characteristics are ascertained via the QC tests before and after the sensors are irradiated to the maximal fluence expectation of $1.2 \times 10^{15} n_{\text {eqv }} \mathrm{cm}^{-2}$ and usually beyond. The charge collection efficiency and strip noise are also measured for the unirradiated and irradiated silicon as the sensor will be glued into a custom connectorized pcb test frame. Irradiations will be conducted cold $\left(<-20^{\circ} C\right)$ to avoid reverse annealing and dry to avoid condensation. The conscripted period $(80 \mathrm{~min}$ at $60^{\circ} \mathrm{C}$ ) of beneficial annealing will be used after the irradiation to simulate intended operation. Front-end bonding during irradiation is also simulated by shorting the strips to each other via a wire-bonded conductor.

\section{Charge Collection Efficiency:}

In this test the charge collection efficiency of the sensor is measured as a function of bias voltage. It is an important test of the bulk radiation damage necessary to ensure acceptable signal to noise ratios in the later years of the experiment. This is a test requiring a subset of the strips to be bonded to an analogue readout chip (eg. the RD collaboration originating ALiBaVa system [86]). Charge liberation in the bulk is achieved using the known betas from a $R u^{106}$ or $S r^{96}$ source. With the analogue chip configured for a 25ns peaking time the collected charge is taken as the most probable value of the induced Landau signal distribution. The expectation is that after $2 \times 10^{15} n_{\text {eqv }} \mathrm{cm}^{-2}$ of neutron irradiation the charge collection of minimum ionizing particle (mip) will still be 7500 electrons or greater. This relates to the 
extremely important signal to noise target (ten to one) for the modules.

Strip Quality vs Bias:

This test involves monitoring the noise levels of all strips on the sensor to observe which are more prone to microdischarge. All strips of the sensor are bonded to analogue or digital readout with $25 \mathrm{~ns}$ peaking electronics. The noise on each channel is monitored as the sensor bias is increased up to or beyond maximal. The microdischarge strip (or strip cluster) is easily identifiable from the discussed striking noise increase $(>100 \%)$.

\section{QA During the R\&D Phase}

This is the current phase of development and evaluation. The QA definition covers optimization of the product design and the manufacturing process. This places much, if not all, of the R\&D phase under its blanket coverage.

It is the QA tests which educate both the designers and manufacturers of any issues in the product and motivate iterations in the design and processing methodology. It is an absolutely necessary aspect of any large project let alone one with the extraordinary challenges offered by the HL-LHC. Organically the nature of the R\&D QA testing evolves over time. Its evolution is in-line with the understanding of the intricacies of the sensor design and silicon response. In its current iteration (as outlined above) the sensor testing regime can be considered quite mature but not necessarily final as R\&D is, of course, still ongoing (see Sections 5.3 and 5.5).

With the advantage of time in comparison to the production phase and sensors that are fabricated only for in-depth investigations, R\&D testing can and should be more thorough. Examples have been presented in Chapter 4 and include a much more extensive radiation campaign involving a variety of sources with a variety of energies and annealing periods. This elucidation of the fluence evolution of the critical sensor properties is obtained with comparable minis. R\&D testing will also include extreme radiation testing at CERN's HiRadMat facility where the conditions of uncontrolled beam splashes during operation are simulated.

Also of significant importance is the detailed investigation of humidity dependency in the leakage current profile and long-term stability. This critical test of the surface geometry and processing is discussed in Section 5.5.3. 


\subsection{Production Site Development}

As one of only four sensor testing sites in the entire ITk Strip project, Carleton University will be responsible for validation of one quarter of the most fundamental component of the detector. This is an important task for the success of the ITk Strip project and requires a high level of expertise. Sites are validated considerably prior to the beginning of production in 2020 and must show a well developed capacity for high volume testing of sensors. Proficiency in the instrumentation, understanding of the setup, and mastery of the test program are required on the experimental side

and must complement a thorough understanding of the sensors being tested. Healthy participation in the R\&D QA testing phase is the natural preparation for such an undertaking and necessitates an even deeper knowledge of the device under test. A good portion of this essential background knowledge has already been presented in Chapters 3 and 4 . The testing procedures were just addressed and in this section discussion begins on the experimental aspects. Prime among the test samples at the Carleton test site are 16 ATLAS12EC, and one ATLAS07. Photographs of these are shown in Figures 5.3 and 5.4 for the A12EC and A07 respectively.

At Carleton, preparations for sensor testing began in the summer of 2016 and mark a milestone in the evolution of the experimental particle physics research regime at the institute. Historically, and more recently with the ATLAS New Small Wheel project, Carleton has been a fabrication and testing ground for gas based particle detectors. Such detectors are good for large coverage and low track multiplicity requirements but solid state detectors with their high granularity and exemplary radiation hardness are the mainstay of the modern high energy physics experiment. With the prescribed ATLAS ITk involvement the physics department at Carleton will become also a fabrication and testing ground for solid state based detectors. A cutting-edge functionality that will prepare the department for involvement in a myriad of physics prospects over the next decades. This lends context to the importance of this Master's work.

Development proceeded from a simple base in both infrastructure and expertise. In collaboration with the Department of Electronics, the Physics Department technical staff resurrected an old cleanroom in the MacKenzie building. The accumulation of necessary knowledge began with expectations of the test procedures gleaned from 


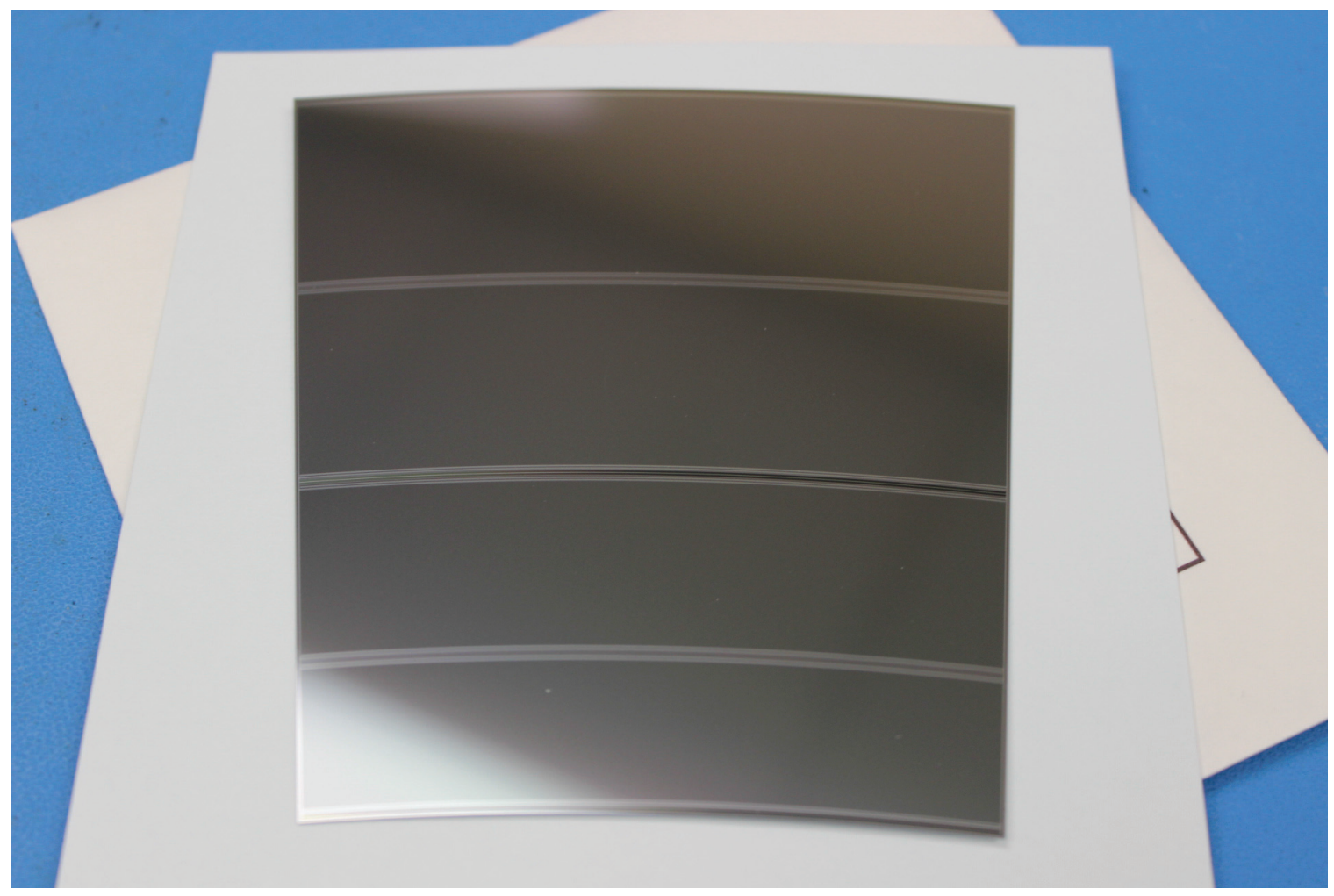

Figure 5.3: Photograph of one of the first ATLAS12EC prototypes ever produced. Taken at Carleton University. 


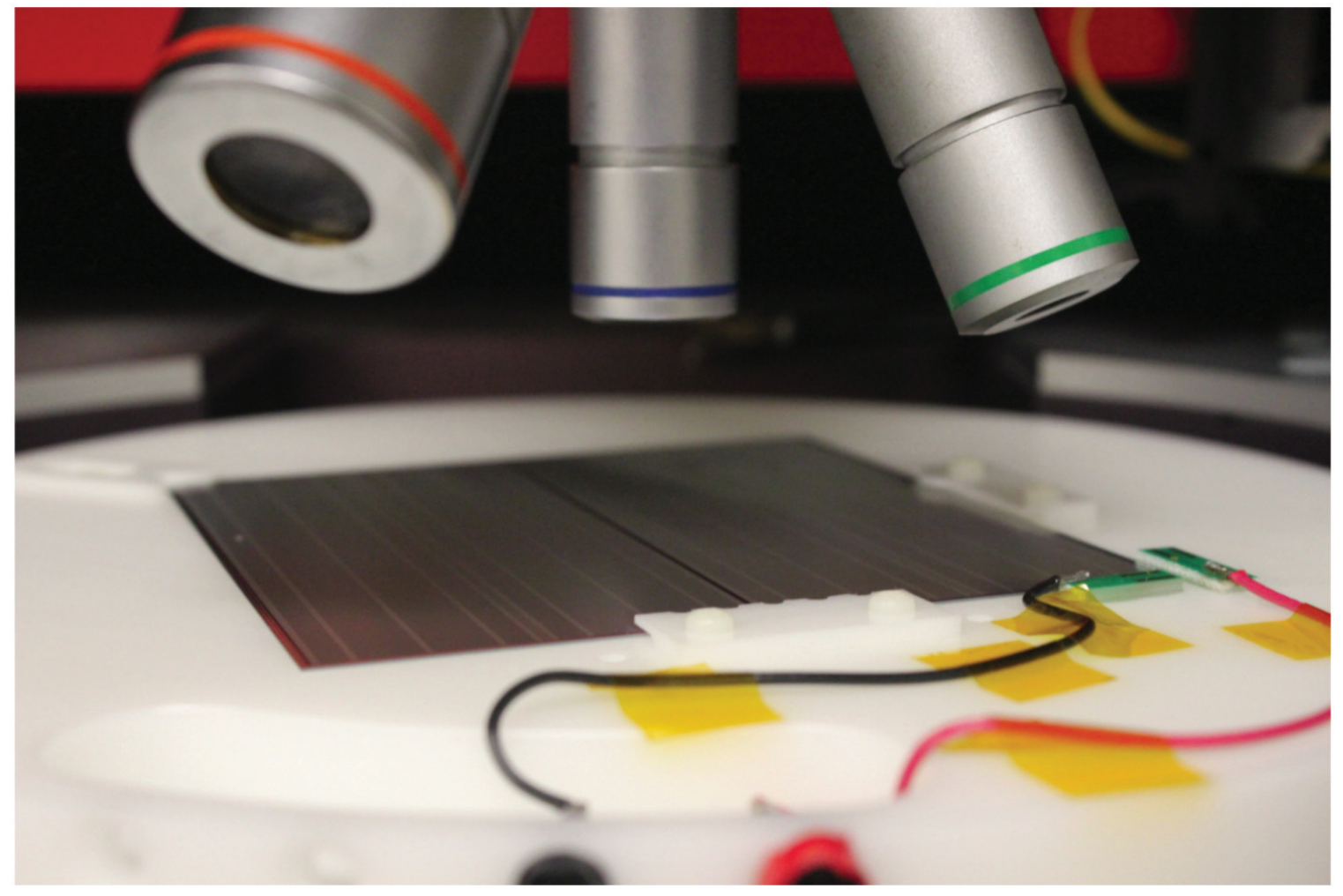

Figure 5.4: Photograph of the ATLAS07 at Carleton University. It is seen wirebonded into the custom jig described in Section 5.4.2 which is vacuum sealed to the chuck of the Karl Suss probestation (Section 5.4.1). 
the technical specifications documents $[30,31,67,81]$. These provide only guidelines for the tests and the expectations were adjusted with a short but extremely informative visit to the lab of the collaborating institute of Cambridge University [50]. Development of the necessary infrastructure began. Instrumentation was acquired through market survey of commercial vendors. Designs and existing instruments were modified to new purposes and settings. Some required components of the setup such as the patch panels and metrology jig were custom designed and fabricated on-site. Collaboration with an Ottawa based microelectronics company, MuAnalysis, was also fostered to allow increased testing capability.

With this work the Carleton site can boast most R\&D QA functionality (Section 5.1.2) and near readiness for production testing. The lack of only a few instruments and designs is already being corrected. All outstanding infrastructure requirements have already been quoted or are in market survey phase. Designs for custom jigs and environmental chambers are under discussion. These are based upon the recent successes of custom components fabricated for the sensor test setup as well as experience that was gained in fabricating a module test stand for DAQ development during the first half of my Master's term. These advances are all that remain for large volume, diagnostical, and irradiated device testing. In other words they are the only outstanding capabilities to address the full canon of tests that is expected of the ITk sensors.

In this way the current site capability could be described as transitory with a bright future ahead. Successful operation of a software controlled, manual probestation based setup to test fifteen R0 prototypes signifies the ability for the full set of QC specified tests (Section 5.1.1). The preliminary focus after the test capability was acquired was the tests that must be conducted on all sensors during production as seen in Section 5.1.1. Results of these tests on both ATLAS07 and ATLAS12EC sensors have been obtained with parallel aspects of the test stand and are the subject of Sections 5.5.1 and 5.5.2. Additional developments in the humidity control and monitoring of the setup have also allowed important observations of leakage current stability issues in the prototypes, this interesting development is addressed in Section 5.5.3.

It is important to note of Carleton's association with the endcap assembly cluster. Having to deal with the fan shape and much increased variability in the endcap 
sensor geometries poses some difficulties that a barrel site would not have to address. Moreover since Carleton will most likely test barrel sensors too, the test stand must accommodate a square sensor too. Mounting mechanisms must be geometrically adaptable. Similarily, probe manipulations become more complex. As well, the multi-contact probe card used in striptests must be unconventionally fan shaped to take advantage of the constant angular pitch of the strips. Finally, the sensor edge processing for the stereo annulus is much more complicated in comparison to the barrel sensor which reduces the yield and implies possibly poorer stability performance (see Section 5.5.3).

\subsection{Mechanical Tests and Considerations}

Mechanical testing conducted at Carleton so far has been restricted to the important QC processes of visual inspection and metrology. Discussion of the infrastructure used for visual inspection is given but it is part of a larger system which is described further in Section 5.4.1. For the metrology case testing is completed at the Ottawa based microelectronics company, MuAnalysis. The instrument used is discussed fully in the sub-section below (5.3.2). In both cases mechanical considerations such as the handling of the sensor during testing and the storage in-between, while seemingly mundane, are necessary technical aspects for any successful sensor testing site (Section 5.3.3).

\subsubsection{Visual Inspection}

A Bausch and Lomb Microzoom I microscope is used with 2x,8x, and 50x objectives as well as an additional 1-2x toggle magnification. This microscope is incorporated in the manual Karl Suss PSM6 probestation detailed in Section 5.4.1. The objective is focused on its movable chuck which has sufficient range for the full sensor surface. An 18 megapixel, USB, and video-enabled OMAX microscope camera was commercially sourced with the optimal optics to minimize vignetting. A high resolution colour video is recorded as a manual scan of the sensor surface is undertaken under magnification of 3-4x. Images of problematic looking areas are obtained at various levels of illumination and magnification. Commercial software provided by the camera manufacturer is used. Figures 4.1 and 5.5 show some examples of visual inspection results obtained 

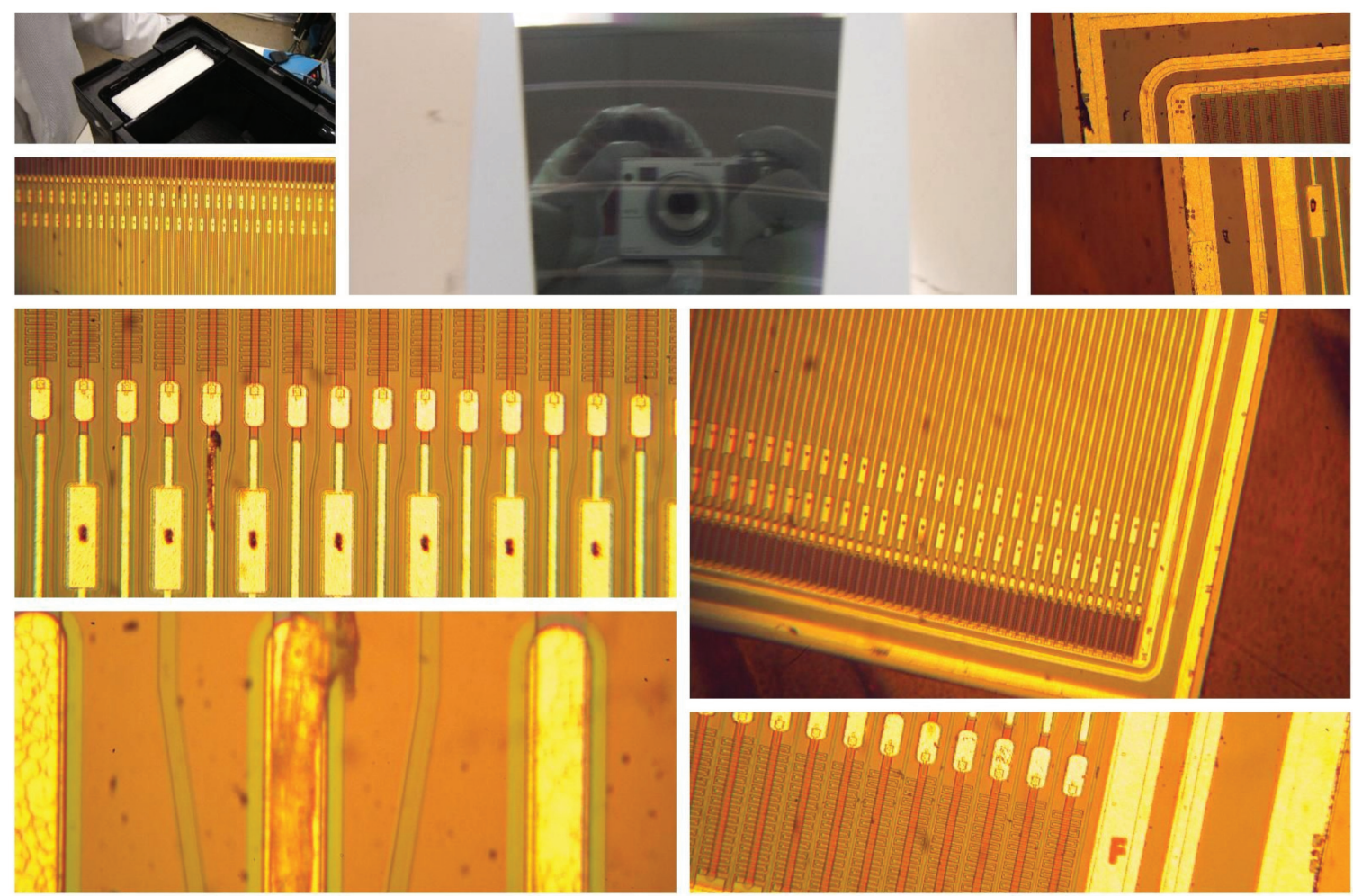

Figure 5.5: Examples of visual inspection results obtained at Carleton. 
at Carleton University.

\subsubsection{Metrology}

Basic metrology of the sensor is carried out at the nearby site of MuAnalysis, an Ottawa based microelectronics company. A Carl Zeiss LSM 310 (confocal laser scanning microscope) with a $633 \mathrm{~nm}$ helium-neon laser is used for a non-contact location measurement of the surface in the prescribed grid (100-130 points per sensor). The instrument's high accuracy stages allow the $\mathrm{x}$, y position to be determined at the micron level. A very high precision (order $0.01 \mu \mathrm{m}$ ) z measurement is obtained using the confocal LSM technique. The laser is scanned in $\mathrm{z}$ with the goal of focusing up to the diffraction limit on the sample. In confocal mode, a pinhole aperture is added at the focal point on the image side, too, lending credence to the mode's name. Another dose of focusing to the diffraction (and manufacturing) limit of the pinhole is required for a sharp image.

During measurement, focus is determined manually due to issues with the autofocus functionality of the manufacturer provided software. The optimal focus and thus most precise $\mathrm{z}$ measurement are observed as the point of brightest contrast in a computer monitor display of the microscope view. This aspect of the test adds a level of subjectivity which lowers the resolution on the $\mathrm{z}$ value. However, conservative estimates still place the $\mathrm{z}$ position resolution obtained at two to three micron, an advantage of the confocal technique. During measurements care is taken to choose points representative of the same sensor surface (passivated or not).

A custom jig was designed and manufactured at Carleton University for use with the MuAnalysis LSM. It offers reproducibility in the xy placement of the sensors and thereby significantly reduces the number of measurements required for the support background. A two body, standoff design is used allowing the top plate's tilt to be adjusted and microscope stage's tilt to be negated (increasing reliability and ease of measurement). A feature of the jig that proves promising for the future is its ability to accommodate all eight sensor geometries of the ITk Strip detector, a first in the collaboration. This feature is seen schematically in the right-side of Figure 5.6, a photo of the jig with an R0 prototype is on the left. Minimal restraint in the vertical direction is used to adhere to the un-stressed requirement.

With the $\mathrm{x}, \mathrm{y}$, and $\mathrm{z}$ data tabulated in an ASCII file, analysis proceeds via the 

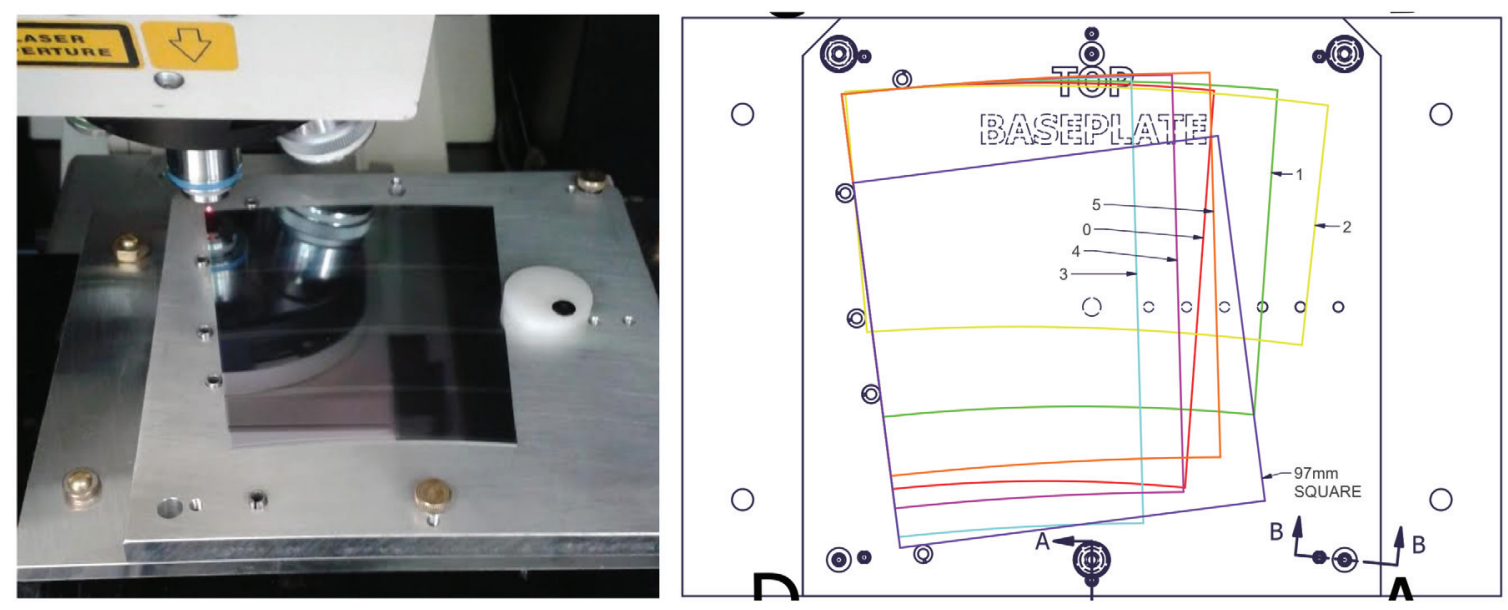

Figure 5.6: The Carleton custom metrology jig featuring an adjustable-tilt two stage design and geometrical accommodation of all sensor geometries. At left; a photo of the jig with an ATLAS12EC in the MuAnalysis LSM. At right; schematic of the geometry accommodation feature.

ROOT software package. A custom script parses the data and produces contour plots of the sensor surfaces. A two edge linear tilt correction is applied in the process by placing three corner points on the same plane. The sensor bow is extracted as the difference between the highest and lowest point on the sensor. Figure 5.7 shows the tilt corrected results for the $16 \mathrm{R} 0$ prototypes. The statistics of these measurements are shown in Figure 5.8. Good consistency was observed for the ATLAS12EC prototypes that were measured. A characteristic igloo-like shape is seen, further study is required before this can be attributed to the sensor or test setup. An average bow of $54.4 \mu \mathrm{m}$ with a standard deviation of $6.5 \mu \mathrm{m}$ was measured for the set. This compares to the value of $(51.7 \pm 12.4) \mu m$ obtained for 100 ATLAS12 sensors [42]. More importantly these are far within the specification of $<200 \mu m$.

\subsubsection{Mechanical Considerations}

The Carleton ITk cleanroom has been maintained since its renewal as a restricted space. Particle surveys conducted at intervals over the intervening year have shown the space more than complies with the ISO6 requirement. A nitrogen system complete with multiple test lines was installed and nitrogen usage rates analyzed to allow prediction of the cylinder exchange periods. Other consumable aspects of the setup 

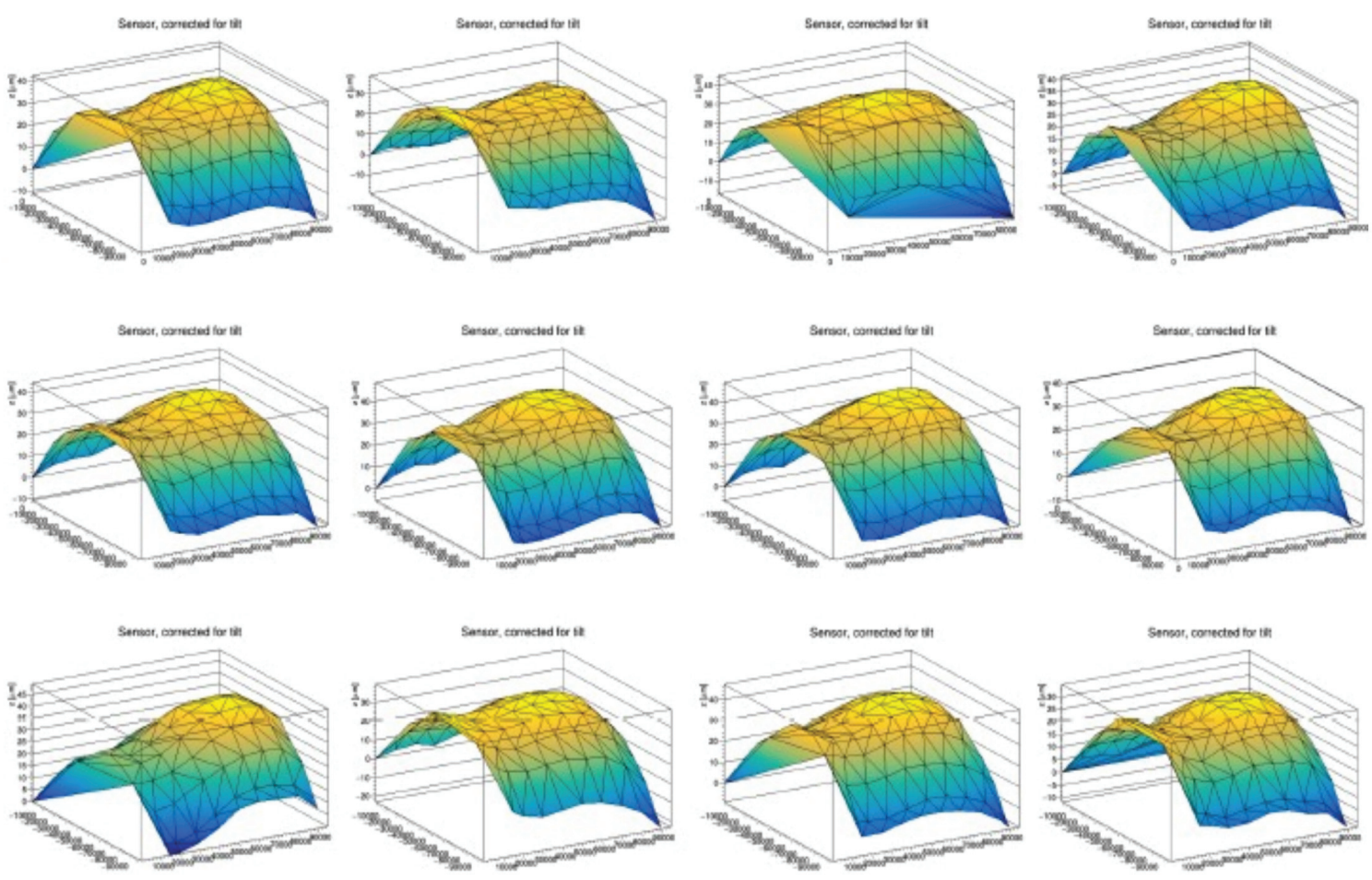

Figure 5.7: Metrology results for sixteen ATLAS12EC prototypes. Data collected at MuAnalysis by Carleton practitioners.

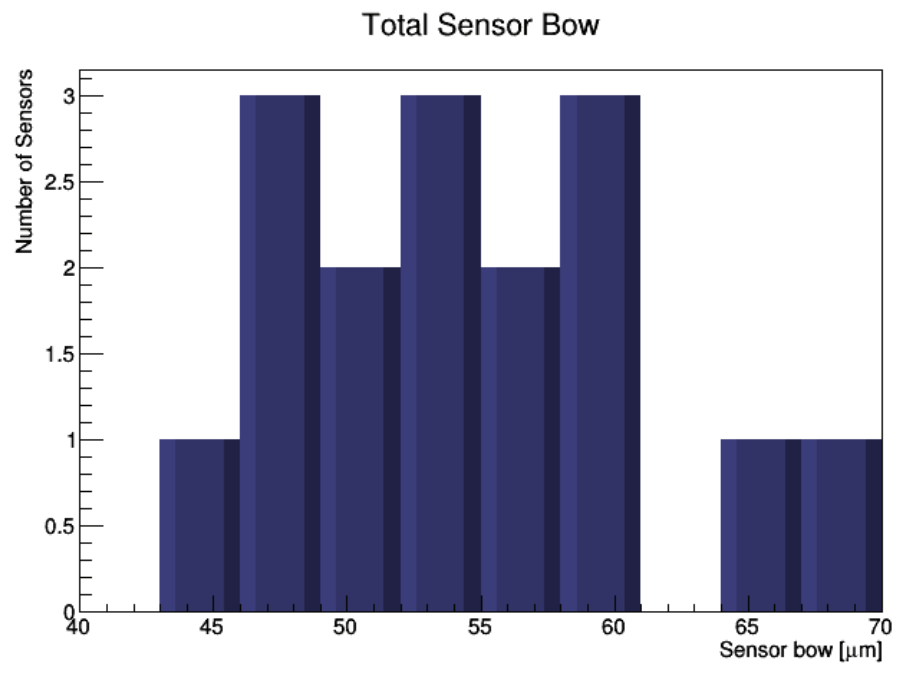

Figure 5.8: Histogram of the ATLAS12EC flatness results shown in Figure 5.7. With a mean of $54.4 \mu \mathrm{m}$ and standard deviation of $6.5 \mu \mathrm{m}$ very good consistency that is well within the $200 \mu \mathrm{m}$ specification was observed. Data collected at MuAnalysis by Carleton practitioners. 
(probe tips, vacuum cups, etc...) are like-wise sourced and maintained.

Environmental monitoring is accomplished by Arduino-based digital sensors, the setup of which is discussed in Section 5.4.4. The Dr. Storage F1-200 dry cabinet for the sensors was acquired commercially after testing of existing infrastructure showed insufficient performance. Using desiccant effected drying the specific model selected is capable of nitrogen free storage at a humidity of $0.1 \%$. The box environment is monitored with built in sensors output via RS-232 to a computer. Currently a rudimentary commercial software is used but outlines exist for a custom LabVIEW implementation with alarm control and integration into the rest of the sensor testing software (see Section 5.4.5).

Handling of the (approximately $10 \mathrm{~cm} \times 10 \mathrm{~cm} \times 0.3 \mathrm{~mm}$, contaminant-reactive, monocrystalline) sensors during testing poses the most sensitive of mechanical risks. Sensors are manipulated using a Sipel TV-1000 bench-top vacuum pen with onetouch release. A non-marking, ESD safe nitrile cup is used. ESD protection is also considered in other features of the test environment such as table top mats and the practitioner's attire. Other aspects of usual cleanroom procedure such as donning of mouth covers for humidity protection are also observed.

\subsection{Electrical Test Setups}

The ITk Strip sensor is a cutting edge solid state device. With its long duration, high impact role in the ATLAS ITk, it is subject to extremely strict restrictions on

batch reliability and consistency, these have been outlined in Section 4.1 and 5.1. The vast bulk of these tests are electrical in nature and use the same or similar aspects of the test stand. In this section an overview of some of these main features and how they have been developed and implemented at Carleton University is given. This infrastructure was either non-existent or in unusable condition at the beginning of this Master's work. Figure 5.9 shows a photo of the electrical test stand at an earlier stage in testing development and shows many of the components discussed in this section.

Electrical connection to the sensor is a sensitive issue. Carleton employs two of the three methodologies mentioned in Section 5.1.1. These are a custom jig design with mechanical restraint and wire-bonded connection to the sensor (discussed in Section 5.4.2) and the pure probestation based connection technique that is required 


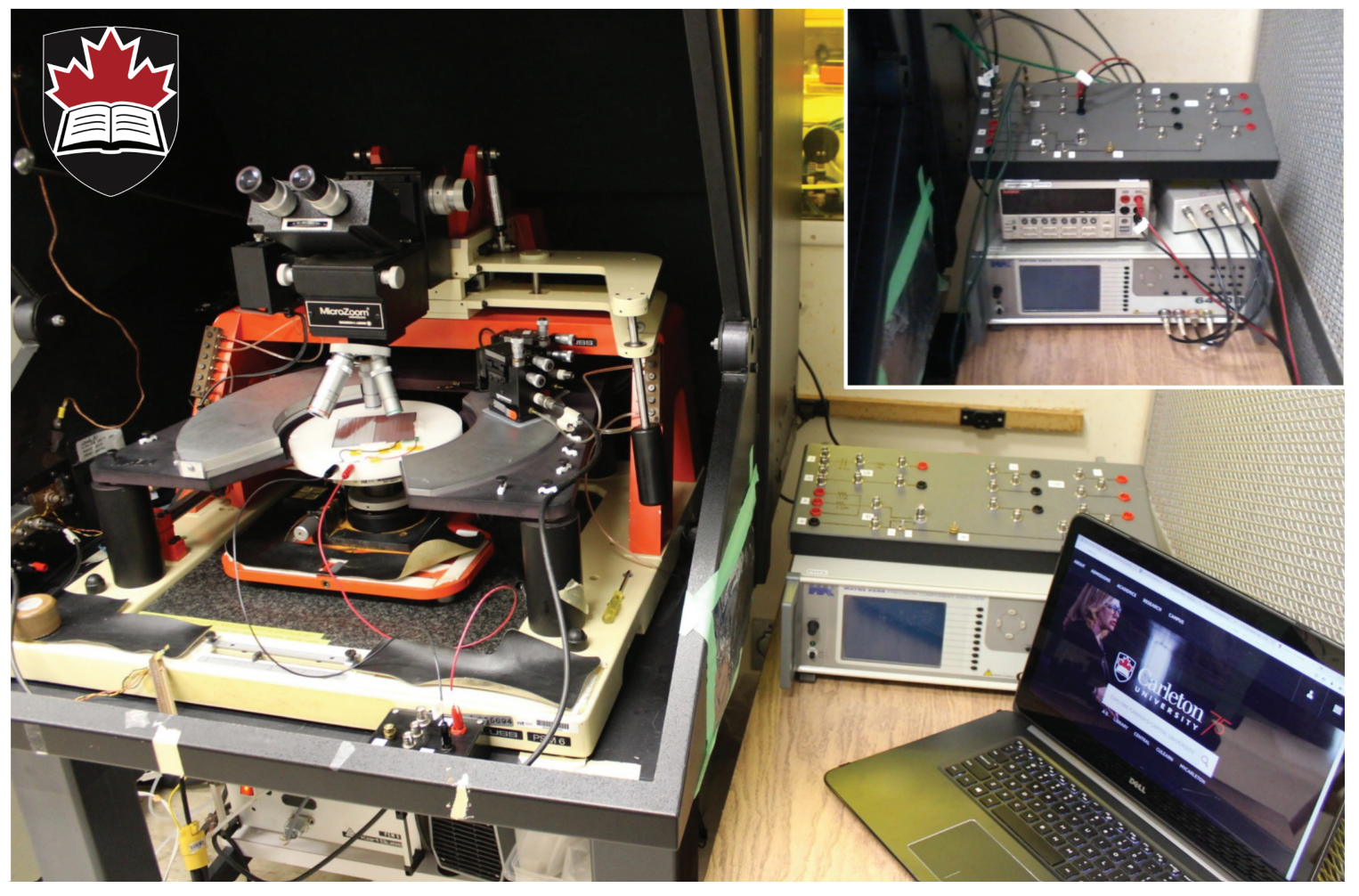

Figure 5.9: The Carleton University electrical test setup at an earlier testing stage in the development. A number of components can be seen; the Karl Suss probestation with the Keithley instruments atop the black enclosure. The LCR meter, largest patch panel, and data acquisition laptop are seen at the right. These components are discussed throughout Section 5.4. 
either way for the strip tests. The probestation, discussed in the next section, was rejuvenated over the course of the past year and new functionality was added. One example of this is allowances for the specific circuitry required by the ITk Strip sensor QA\&QC program. Some of the equivalent circuitry can be seen in Figures 5.1 and 5.2. This basic knowledge while overviewed in technical specifications [30,31, 67, 81] required a good deal further development from much more difficult to find sources such as accounts of tests performed at other institutes. Once the subtleties were determined a number of patch panels were designed and fabricated (on-site) to bring them to reality in an efficient manner for large volume testing (Section 5.4.3). Other instruments and monitoring functionality were obtained through market survey or developed from simple commercial components (Section 5.4.4).

With the large sensor throughput needed during production, procedures must be automated to a great deal and a large variety of software is used to across the test setup. The vast majority of tests will be conducted through a suite of LabVIEW virtual instruments but the variability of tests and infrastructure has necessitated development in six programming languages. Software is discussed in Section 5.4.5. Long-term testing and environment is then discussed in Section 5.4.6. Finally, in section 5.4.7 an outline of some of the crucial understanding gained in operation of the newly developed test facility is given.

\subsubsection{Probestation}

Most electrical tests are conducted in the probestation; a standard tool of the microelectronics industry. The system consists of a movable chuck on which the sample is placed. The chuck usually also has z control to allow sample loading. A platen sits with its surface slightly above that of the loaded chuck's and supports the probe assemblies. It has a large aperture through which the probes can be lowered. The probe assemblies (termed micromanipulators) consist of micron scale needle tips supported, mechanically and electrically, by an arm which is controlled via high precision counter-turned screws in the $\mathrm{x}, \mathrm{y}$, and $\mathrm{z}$ axes. Critical for placement of the probes is a microscope which is focused on the chuck surface. It must have $\mathrm{x}, \mathrm{y}$, and $\mathrm{z}$ control to allow multiple probe placement and sample height variation. The entire probe environment must be contained in a light tight enclosure with bulkhead connections for services and electronics. Finally, to isolate the action of probing from any mechanical vibrations in the room an isolation table should be used. 
At Carleton the device currently in use is the Karl Suss PSM6 manual probestation. It is the large black and red apparatus in on the left in Figure 5.9. Manual in this case refers to the control of chuck and microscope. The sensor samples are placed directly on the electrically insulated kapton covered chuck and restrained by the probe contact. Alternatively, they are held in the custom jig (see below) mounted on the chuck. The chuck used in the PSM6 probestation features a vacuum capability, is ultra-flat ( $8 \mu \mathrm{m}$ tolerance), and can scan an area much greater than that of any of the sensors surfaces. The station is equipped with five micromanipulators with resolution of eighty turns per inch (roughly $5 \mu \mathrm{m}$ ). Two sizes of tungsten probe tips are used; $12 \mu \mathrm{m}$ and $25 \mu \mathrm{m}$. Despite advantages in damage to the sensor, contact reliability deteriorates as the diameter of the probe tip is reduced and the smaller tips are reserved for the DC pads only. The microscope in place is the original model that was installed in the probestation, it was described in Section 5.3. The enclosure and pneumatic vibration isolation table were likewise in-place when the probestation was acquired.

Carleton University is currently in the market survey stage for acquirement of a semiautomatic probestation. This is the most significant piece of the infrastructure still required. Aside from the large volume capability offered by its more automated nature (semi- refers to its manual wafer loading) this device will become a very powerful diagnostic tool by incorporating some exciting functionalities. An IR camera will be added in conjunction with the probestation microscope allowing microdischarge detection. This feature promises advancement in the understanding of processing issues as well as issues that may be more endemic to the sensor design (see Section 5.5.3). Also included is a small environmental enclosure at the probe surface. This will not only allow greater resolution in the thermal and humidity responses of the sensor characteristics but probe testing of irradiated samples (which must kept cold during testing for annealing considerations). This machine will be incorporated into the LabVIEW software suite. It is expected for delivery by the end of the calendar year.

\subsubsection{Custom Jig}

Due to its ease of use and unobtrusiveness, vacuum had long been the preferred method for mechanical restraint of the sensor. However, during the ATLAS07 sensor iteration collaborating institutes observed a susceptibility to micro-cracking of the 
sensor when subject to chuck vacuum. A jig with restraint via mechanical fixtures was designed. It incorporates the fact that the bias connections to the sensor bulk are permanent during the test regime and includes circuitry for a wire-bonded connection to the sensor bulk. For use at Carleton the basic jig design sourced in the collaboration was modified for compatibility with the chuck of the Karl Suss probestation (a requirement for strip testing). The plastic jig body was manufactured to strict tolerances on campus at the Carleton University Science and Technolgy Center (STC) using their five axis water-jet cutter. The simple on-jig circuitry was custom fabricated and mounted after reception from the STC. Wire-bonding of sensors into the jig is accomplished using a Department of Electronics Kulicke\&Soffa 4524-D manual ball bonder with $25 \mu \mathrm{m}$ diameter gold wire.

The Carleton University custom jig is featured in Figure 5.10. The ATLAS07 sensor is seen wire-bonded into the jig on the left. While future iterations will incorporate the geometrical adaptability of the metrology jig design its current realization can accommodate barrel sensors only (see Section 5.3.2). Delrin plastic is used to provide sufficient isolation for the backplane at high voltage. The back of the jig is patterned with that of the Karl Suss chuck's vacuum groove, a modification to the original design. This mating is what drives the circular shape. An annulus overhang with a cut-out sections ensures the jig sits snugly on the chuck surface and accomplishes positioning and orientation. A custom fabricated large diameter o-ring is used for the vacuum seal. The jig was also manufactured to strict tolerances in flatness and parallelism to try and provide the optimal surface for large area automated probing. Coupling these requirements with a plastic material was not a trivial matter and necessitated special care in the fabrication process (eg. rod source delrin, precise cut order). The sensor is restrained using three low profile delrin holders. A loose spring behind a holder in one of the corners confines the sensor in $\mathrm{x}$ and $\mathrm{y}$ against holders on the opposing sides. The faces of the holders are angled and loosely restrict the sensor edges in the z direction. Two tabs of PCB with gold plated copper tracings/pads are epoxied to the jig and are used for wire-bonding. Soldered connections are routed through a cutout to the connectorized edge of the jig. The corner with both edge holders is used for wire-bonding as it offers the most reproducible xy corner position. 

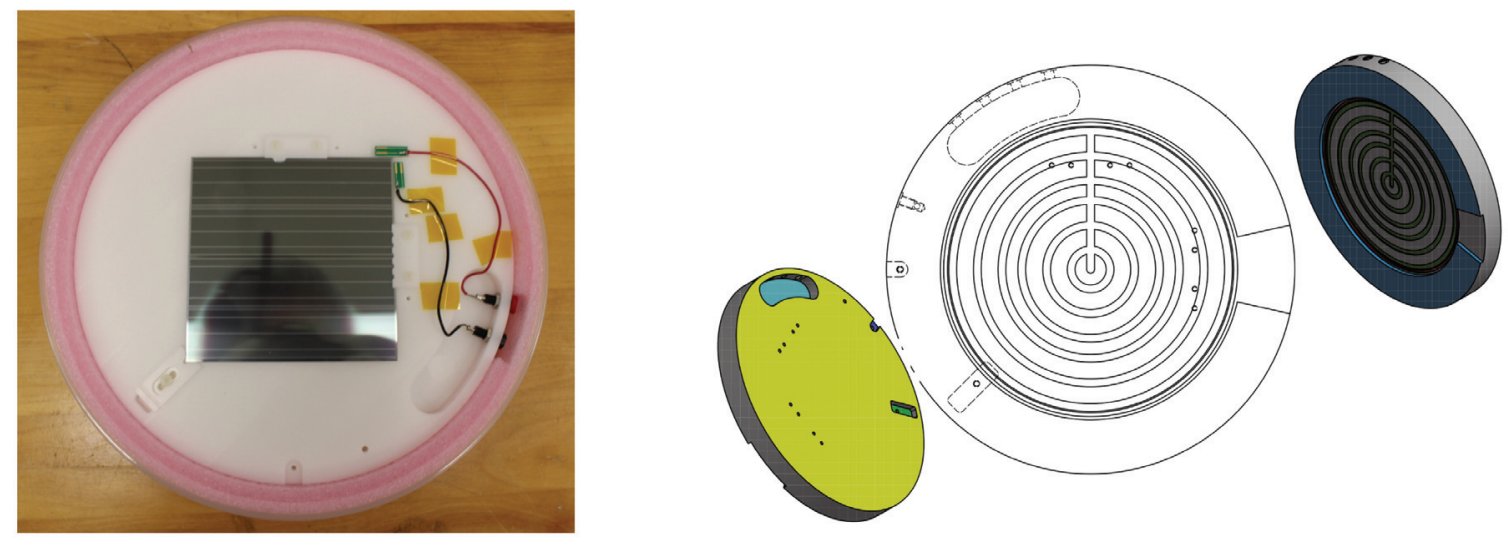

Figure 5.10: The Carleton University custom test jig. Designs modified and jig fabricated on-campus. Left; photo of the jig with the ATLAS07 short strip barrel prototype wire-bonded in. Right; schematics of the design developed with the Physics Department design staff.

\subsubsection{Patch Panels}

As mentioned, technical outlines of the tests exist in the specifications of the recent sensor iterations (A07-A17LS $[30,31,67,81]$ ) however they do not include all specifics of the circuitry. Observations of the working test site at Cambridge University [50] allowed the specific components and connections that were glossed over in the specs to be ascertained. Nonetheless, further research was required due to the constraints of differing test apparatus. In the end the equivalent circuitry for the full set of $\mathrm{QC}$ tests [30,31] was produced and subsequently shared with the other production and testing sites. Examples of these circuits are shown in Figures 5.1 and 5.2. The method for realizing these circuits in reality that will be used at Carleton was also shared (the patch panels). Together these allow for quick acclimatization to the technical aspects of the tests for any new collaborating institutes.

The physical implementation of the circuits is achieved with a series of four patch panels which are outlined below and include the required added allowances for a manual probestation. The number of sensors and long time-scale of the testing program oblige a stable and easy to use circuitry platform and this is actualized with this custom set, partially pictured in Figure 5.11. The four patch panels are dispersed in the test stand with the general purposes of adapting connectors and locating the circuitry. Inside the enclosure are the two smaller boxes in Figure 5.11. The long narrow box is used to make connections as close to the sensor sample as possible (as 


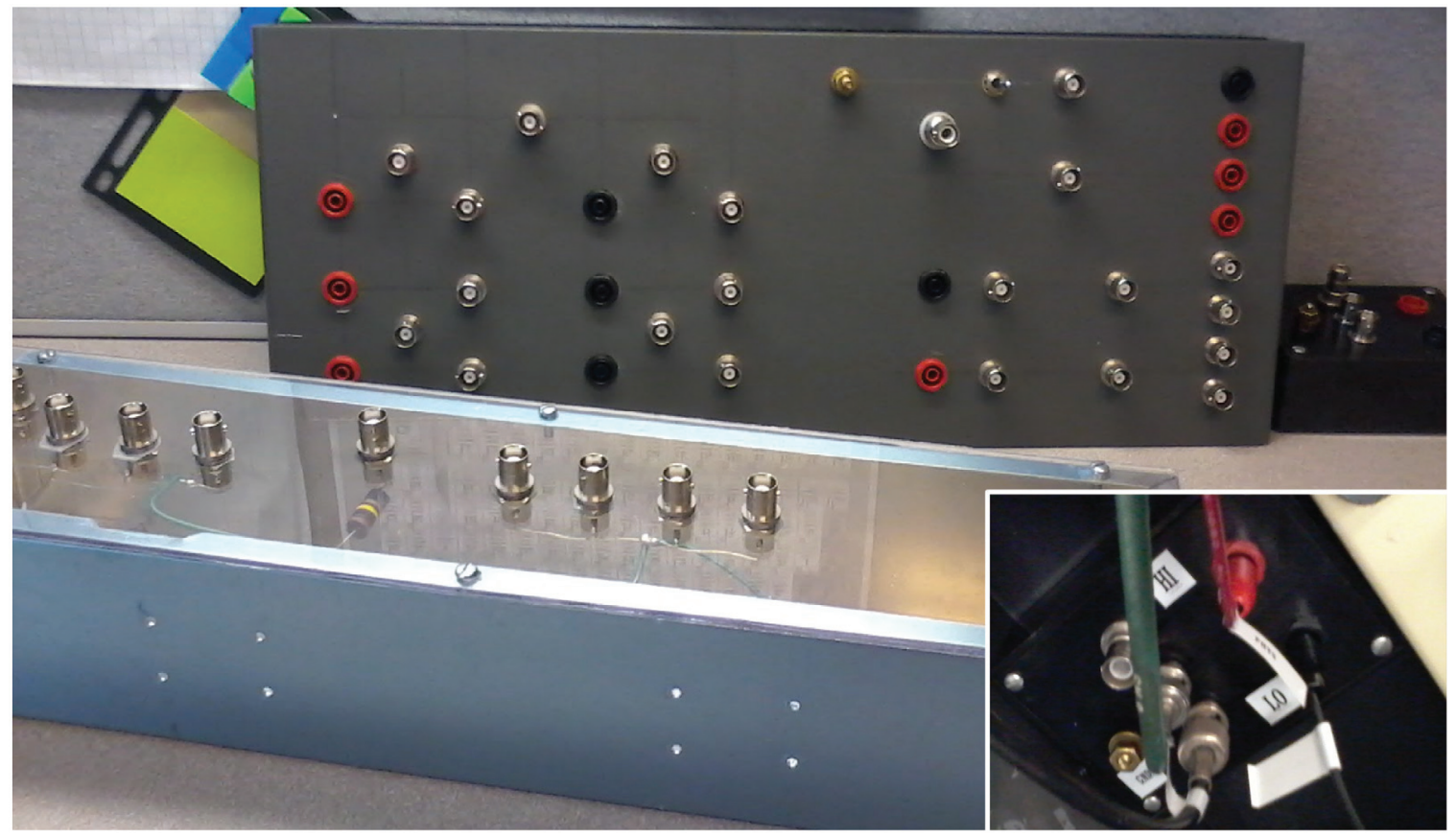

Figure 5.11: The custom designed and fabricated patch panels in use at the Carleton University sensor test facility.

desired for the capacitance measurements). The small black box serves as an adapter unit for connections to the custom jig. The largest box is placed outside the enclosure and allows the manual switching of circuits without having to open the probestation (sufficient switching capability for a manual probestation setup). The set is rounded out with a bulkhead panel for the Karl Suss enclosure (not shown). Grounding has been specially considered due to the capacitive nature of the sensor.

The patch panels were designed, missing components sourced, and then were fabricated in the physics department workshop. Connection schematics for each QC test were generated allowing easy operation by sensor test operators. The corollary patch panel schematics to Figures 5.1 and 5.2 are given in Figure 5.12.

\subsubsection{Instrumentation}

All of the fundamental measurement instruments for the QC tests were sourced through market survey and installed in the cleanroom. This includes the high voltage picoamp resolution source measurement unit (SMU) that is used for sensor bias. 


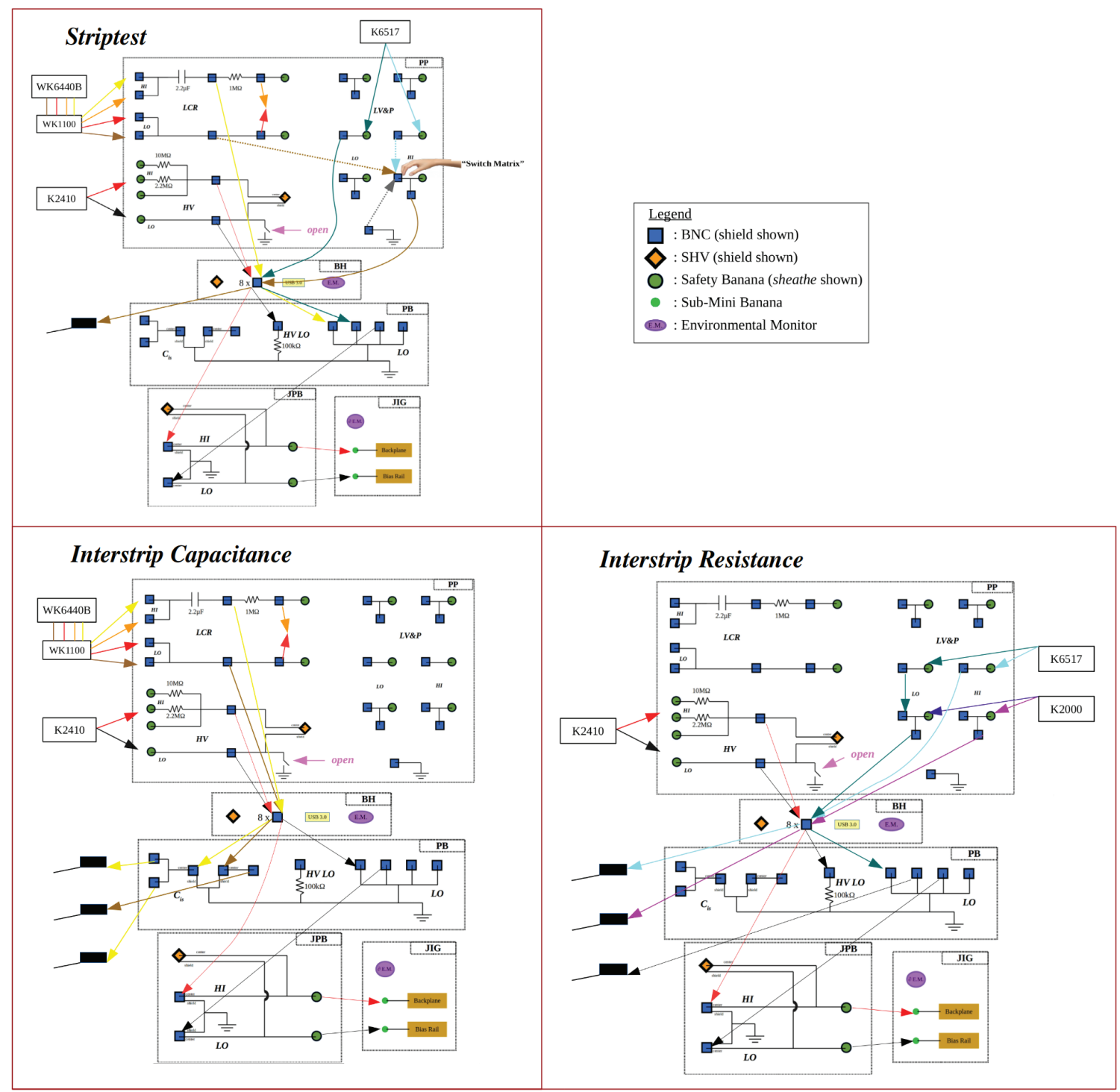

Figure 5.12: Examples of the patch panel connection schematics produced for the QC tests given in Section 5.1.1. Top; full striptest (corollary to equivalent circuit of Figure 5.1). Centre; interstrip capacitance connections (Figure 5.2 left). Bottom; interstrip resistance (Figure 5.2 right). 

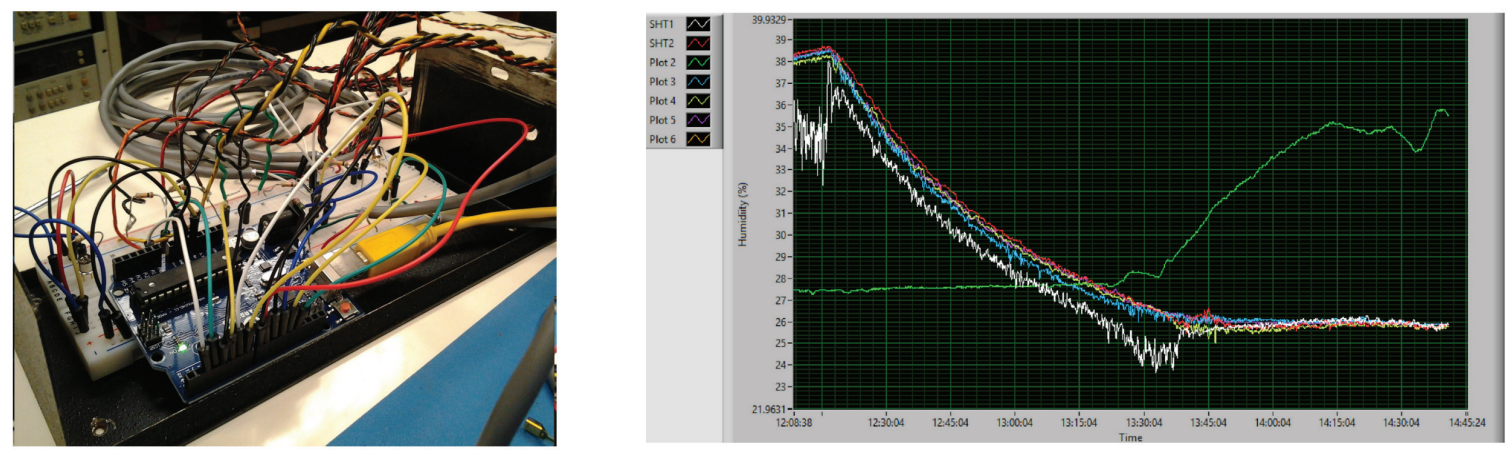

Figure 5.13: Examples of the Arduino based environmental monitoring system. Left; photograph of the mostly breadboard based circuitry used in the six SHT setup. Right; example results of humidity over time in the newly developed LabVIEW code. The green line represents an SHT outside of the enclosure.

The Keithley 2410 is chosen in this regard due to its exemplary compliance limiting functionality. The high impedance devices required for the ultra-sensitive interstrip resistance measurement have also been obtained. A Keithley 6517 SMU and Keithley 2000 multimeter were chosen. For the AC measurements requiring sub-picofarad resolution, a state-of-the-art Wayne Kerr 6440B with femto-farad resolution was acquired.

For environmental monitoring during testing a custom Arduino-based setup is used. Sensirion SHT75 digital temperature and humidity sensors are used as is a Vishay BPW24R silicon photodiode for light level measurement. Setups using up to six sensors have been operated successfully. Custom Arduino, C++/ROOT, python, and LabVIEW code was written for control, readout, and plotting of the data in Linux and Windows environments. All components were selected and acquired commercially with the simple circuitry needed fabricated in house. Shown on the left side of Figure 5.13 is the semi-breadboard based circuitry implemented for the six SHT environmental monitoring setup. On the right side is an example of the type of result obtained in the newly developed LabVIEW code. Custom pcb adapter boards will eventually replace the breadboard after the full set of Arduino based sensors is decided. 


\subsubsection{Sensor Test Software}

The main software is a custom suite of over 1000 LabVIEW virtual instruments. It is used for control of the electrical test stand. The program suite is very advanced; inherited from production testing of the SCT with updates for the new detector fabrication already partially realized. It is of modular design. The software's main functions are data acquisition, instrument slow control, and safety interlocking. All electrical QC tests (Section 5.1.1) are implemented in the software with the exception of strip/implant resistance and PTP structure investigation. A number of common SMUs, voltmeters, and CV meters are likewise already substantiated in the software as is the application program interface (API) for a semiautomatic probestation. General purpose interface bus (GPIB) control using the standard commands for programmable instruments (SCPI) language is used for the majority of instrument slow control and allows software-instrument integration built from scratch when a relevant API is not provided. The instrumentation is not only remotely controlled along the testing procedures but set-up to accommodate different failure modes (eg. immediate rampdown of bias voltage after compliance is hit).

The software is a sensitive aspect of the setup since it controls the vast majority of instrumentation. As such, a high level of understanding of the complex system is required just for its safe operation. Further insight is needed of the structure, dependencies, and technicalities to implement the software at a new site such as Carleton since no documentation is existent. Finally, for modifications such as implementation of new instruments, new (endcap) sensor geometries, or adaptation to differing test setup capabilities (eg. manual versus semiautomatic probestation) an extremely high grade understanding is needed. At Carleton the sensor testing LabVIEW has been successfully initialized, modified, and used for testing of ATLAS ITk prototypes. Modifications for added data saving functionality, new testing modalities, and sharper control of the CV meter have been actualized with the only remaining hurdle of manual probestation implementation outlined in-depth.

\subsubsection{Long-Term Testing Considerations}

Long-term leakage current stability testing is an extremely sensitive diagnostic test as discussed in Chapters 3 and 4 as well as in the QA Section above (5.1.2). Moreover with the new and more complicated geometry of the ATLAS12EC the issues in 
surface processing that this test reveals may be more prominent. This is one of the major concerns for this iteration of testing and development. The duration and environmental conditioning used in the long-term testing of leakage current necessitates inclusion of some setup aspects not yet discussed.

Techniques to allow the use of the vibration isolation table system for the multihour tests despite issues with its basic functionality were developed, tested, and are implemented to improve the setup's reliability. The temperature and especially humidity conditions have significant consequences for the charge concentrations on the surface interfaces as well as current flow in the bulk. For this test to be conducted in a meaningful manner reasonable environmental control and monitoring is required.

Monitoring is accomplished via the Arduino and SHT based systems. Several methodologies for humidity control were developed and tested and offered improvements in the stability of the engendered humidity (variations of 10-15\% relative humidity over 24 hour tests improved to a couple of percent). A LabVIEW program for control of the humidity (via a programmable-SMU controlled fan) was written from scratch using the SCPI protocol. It features functionality for long-term hands-free operation. Coupled with the sensor testing and environmental monitoring LabVIEW codes this allows the long-term tests to be maintained remotely, an important logistical consideration. Modifications to the sensor testing code were made and allowed the combination of all data streams into one plot via a custom written $\mathrm{C}++/ \mathrm{ROOT}$ code. Results from this setup are the subject of Section 5.5.3.

\subsubsection{Procedure}

Electrical testing constitutes the majority of the sensor test program and it is important that this aspect of the site setup is understood completely. The numerous high voltage tests and high sensitivity probe tests must be conducted safely and with very high reliability. At Carleton, understanding of the electrical test stand has advanced far over the year since the effort began. Debugging of faulty connectors and manipulators, and of isolation and grounding issues has been conducted successfully. Techniques and procedures to minimize mechanical and electrical stresses on the sensor have been developed. As have workarounds to temporary lapses in the infrastructure capability (such as the manual probestation's vibration table). Valuable experience with contacting the sensor via probe needle has been gained with observable consequences for the reliability of the test results and well-being of the 
silicon.

This accumulation of technique and procedure will become the basis for a set of operating principles. Eventually yielding documents which future sensor test operators (students and production testing technicians) will use in the day-to-day operation of the Carleton University test site.

\subsection{Electrical Test Results of ITk Strip Sensors}

This section displays most cogently the basic readiness of the Carleton University testing site. Here the recently obtained results for the leakage current and depletion voltage of the ATLAS12EC are presented. The ATLAS12EC is the first stereo annulus sensor ever produced and the results shown are the some of the first ever conducted on this promising sensor design. The consequences of the test results are thus significant for the endcap in particular and the ITk Strip design in general as the novel device implementation must be thoroughly qualified to be used.

Three tests are discussed; the IV curve in Section 5.5.1, the CV curve in Section 5.5.2, and long-term leakage current stability in Section 5.5.3. The ATLAS12EC are the most recent members in the long, rich lineage presented in Chapter 4. As such the IV and CV curve results obtained are directly compared to their literature counterparts for the ATLAS07 and ATLAS12 submissions [42,45]. Similar scientific articles are expected to be produced for the ATLAS12EC by the end of the year. My results will feature large in such documents since the fifteen R0 prototypes tested at Carleton is nearly twice that tested at any other institute.

\subsubsection{Leakage Current and the IV Curve}

The importance and consequences of the sensor's reverse bias leakage current have been discussed throughout Chapters 2, 3, and 4. Its roles are multi-fold and are amplified by the degenerative effects of radiation. At the sensor level it influences noise and instability likelihoods, and is fundamentally related to the depletion capacitance and resistivity of the silicon. While, at the detector level the sensor bias' leakage current is associated with large power consumption.

IV curves are conducted in the test stand outlined above. Both methods of sensor contact have been used in successful testing of barrel and endcap sensors. In the case 
of probe needles, sensor contact is diagnosed using repetitive capacitance measurements with the CV meter (switched in using the patch panel outside the enclosure). The Keithley 2410 SMU with high voltage capability, picoamp resolution, and exemplary compliance limiting functionality is used to apply the sensor bias. With the sensor photo-sensitivity the enclosure must remain closed for the duration of the test and the electrical connections are routed through the custom bulkhead panel. Conventional ramp parameters used are $10 \mathrm{~V}$ steps, with 10 s settle period before current measurement. The sensors are ramped from a reverse bias voltage of zero up to a maximum of 1000 volts. Compliance limiting on the order of $1 \mu \mathrm{Acm}^{-2}$ is used. Temperature and humidity data is recorded at up to six locations in the probestation enclosure using the multi-SHT Arduino-based setup.

Its semiconductor nature implies that the silicon electrical character will be extremely temperature dependent. The well-established form of the leakage current dependency is discussed in Section 3.3.4 and summarized by Equation 3.6. Temperature conditions which could be expected in a clean-room environment $\left(20-30^{\circ} \mathrm{C}\right)$ can produce current variations on the order of $100-50 \%$. Thus, for a meaningful comparison, results must be temperature normalized. Figure 5.14 shows the effect of the temperature correction on ATLAS12EC IV curve data collected at Carleton. The top plot shows the uncorrected IV curves taken at temperatures in the reange of 21 to $28.5^{\circ} \mathrm{C}$. The bottom plot shows the same data with the temperature normalization to $20^{\circ} \mathrm{C}$ applied. An attempt to accentuate visualization of the effect is made by using the same linear y-scale in both plots.

A large number of IV curves have been conducted at Carleton using different samples, connection techniques, sample IV histories, humidities, temperatures, and sample humidity histories. The largest set of measurements was the initial characterization of the first batch of R0 prototypes in April of 2017. Fifteen full-size ATLAS12EC sensors were measured. Temperature and humidity are monitored but not controlled for the first tests of each sensor.

The $20^{\circ} \mathrm{C}$ normalized results are given in the bottom plot of Figure 5.15. IV curves for batches of 33 full-size ATLAS12 (middle plot) and 19 ATLAS07 (top plot) are also given for comparison. For the most part the IV curve shapes for the unirradiated large-size ITk prototypes are ideal, displaying stable plateaus and an observable full depletion voltage kink (caused by the depletion expanding through the $p^{+}$backplane 

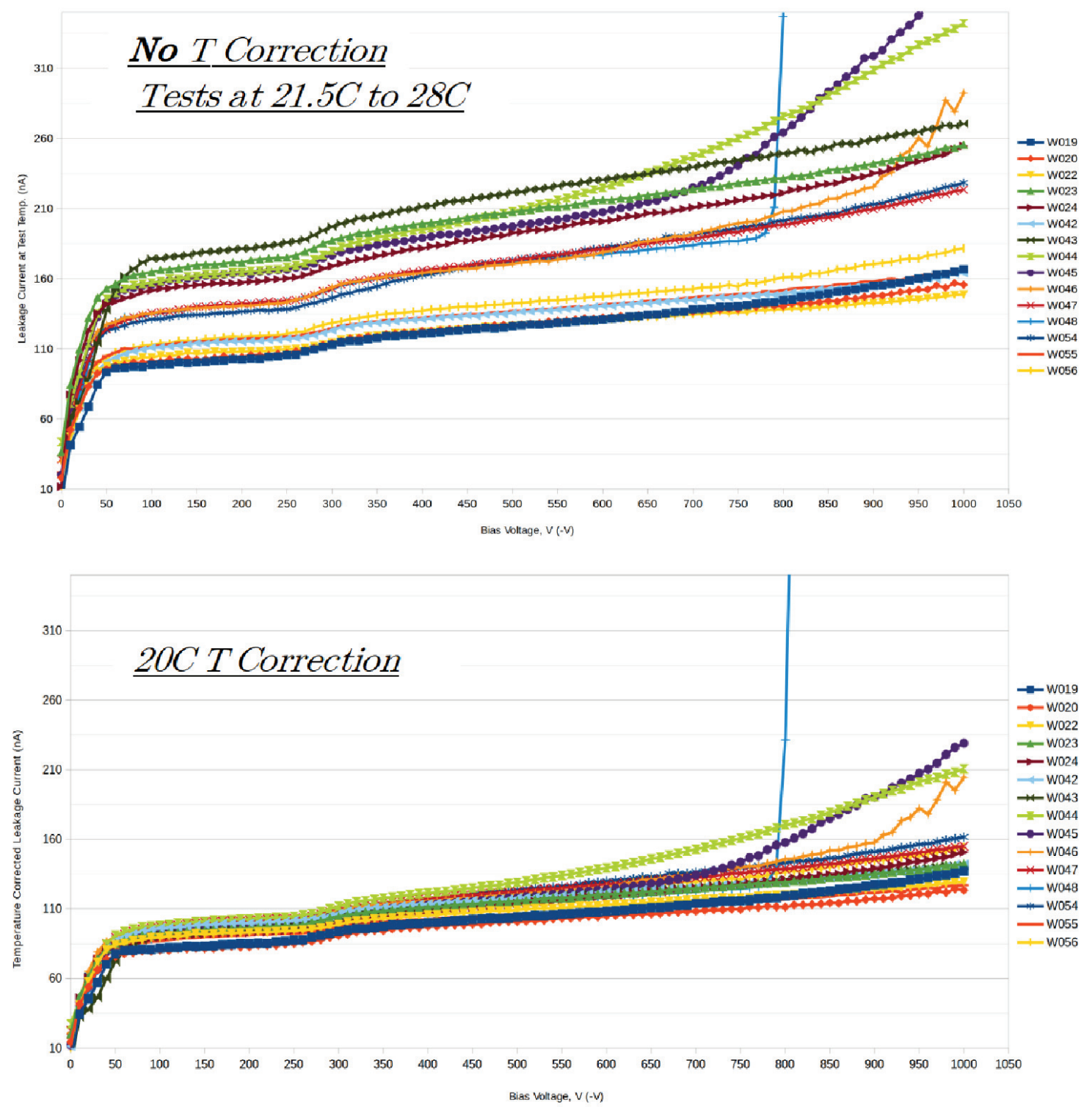

Figure 5.14: Visualization of the effect of $I_{\text {leak }}$ temperature correction using ATLAS12EC data obtained at Carleton University. Top; IV curves obtained at $21-28.5^{\circ} \mathrm{C}$. Bottom; the same data normalized to $20^{\circ} \mathrm{C}$. An attempt to use equivalent linear y-scales has been made. 
and edge metal). The ATLAS12 and ATLAS12EC show an extremely good consistency across the batch which is not observed for the ATLAS07 (although only small samples are given). For the slim-edge, stereo annulus ATLAS12EC this is good sign indicative of quality silicon and reasonable edge processing.

Supporting the claim of better than reasonable edge processing are the low rates of microdischarge that are observed for the given sample sets (one sensor in each). Again, the argument is strengthened for the endcap sensor. Leakage currents are otherwise within specification $[30,67,81]$ by orders of magnitude. The histogrammed results are given in Figure 5.16. For the Carleton data an average leakage current of $122 \mathrm{nA}\left(1.36 \mathrm{n} \mathrm{cm}^{-2}\right.$ ) at $600 \mathrm{~V}$ (or microdischarge onset), and $20^{\circ} \mathrm{C}$, was obtained with a RMS error of $8 n A\left(0.09 n \mathrm{Acm}^{-2}\right)$. This compares to the specification of $179.9 \mu \mathrm{A}$ $\left(2 \mu \mathrm{Acm}^{-2}\right)$ [30].

The ATLAS ITk sensor design is observed to have a marked humidity sensitivity. Microdischarge onset voltage and leakage current stability are most affected while the level of leakage current itself remains relatively unchanged. This is in-line with the effects of mobile ions in the silicon dioxide surface as presented in Section 3.3.6.

This behaviour has been observed in the ATLAS12EC submission as well, as is seen in Figure 5.17. Here the temperature normalized IV curve measurements of one full-size R0 prototype are shown at various humidities. The inverse relation between microdischarge onset voltage and humidity is obvious. Further focused investigations into the dependency of leakage current and breakdown onset voltage on humidity and the sample's mechanical and electrical history have been conducted using IV curves and long-term leakage current stability measurements. These results are presented in the Section 5.5.3 below.

\subsubsection{Full Depletion Voltage and the CV Curve}

As discussed in Sections 2.7, and Chapter 3 the full depletion voltage is an important characteristic related to the active depletion depth and thus size of a generated signal. It is adversely affected by the irreversible changes in effective charge carrier density that are associated with the accumulation of bulk defects due to irradiation. With the fluences and silicon resistivities projected for the ITk, operation in a partial depletion state is expected towards the end of the experiment lifetime [54] and the importance 

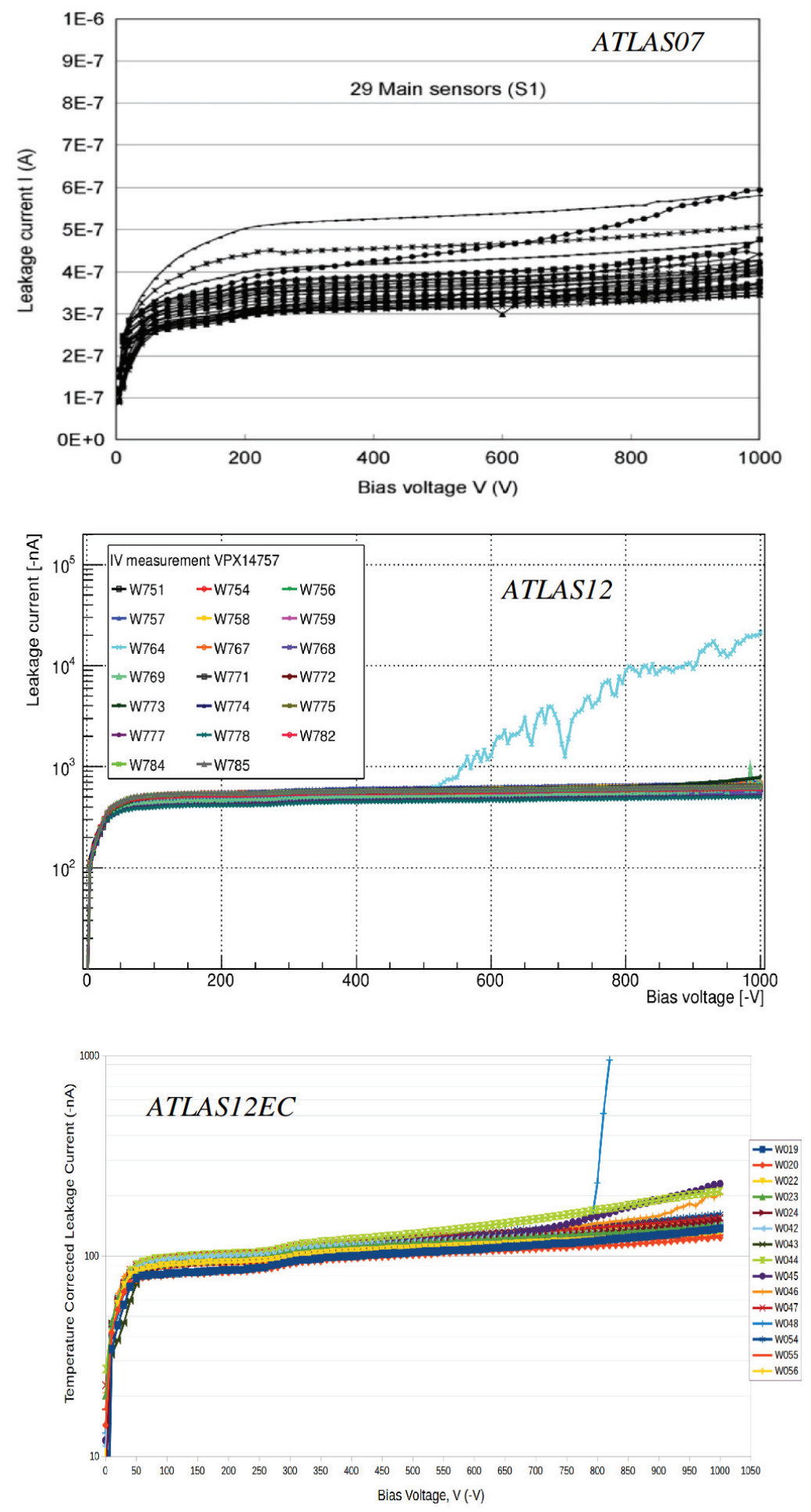

Figure 5.15: A comparison of temperature normalized IV curve results for the three most recent sensor submissions. Top; ATLAS07 [44]. Centre; ATLAS12 [1,42]. Bottom; ATLAS12EC (data obtained at Carleton). 

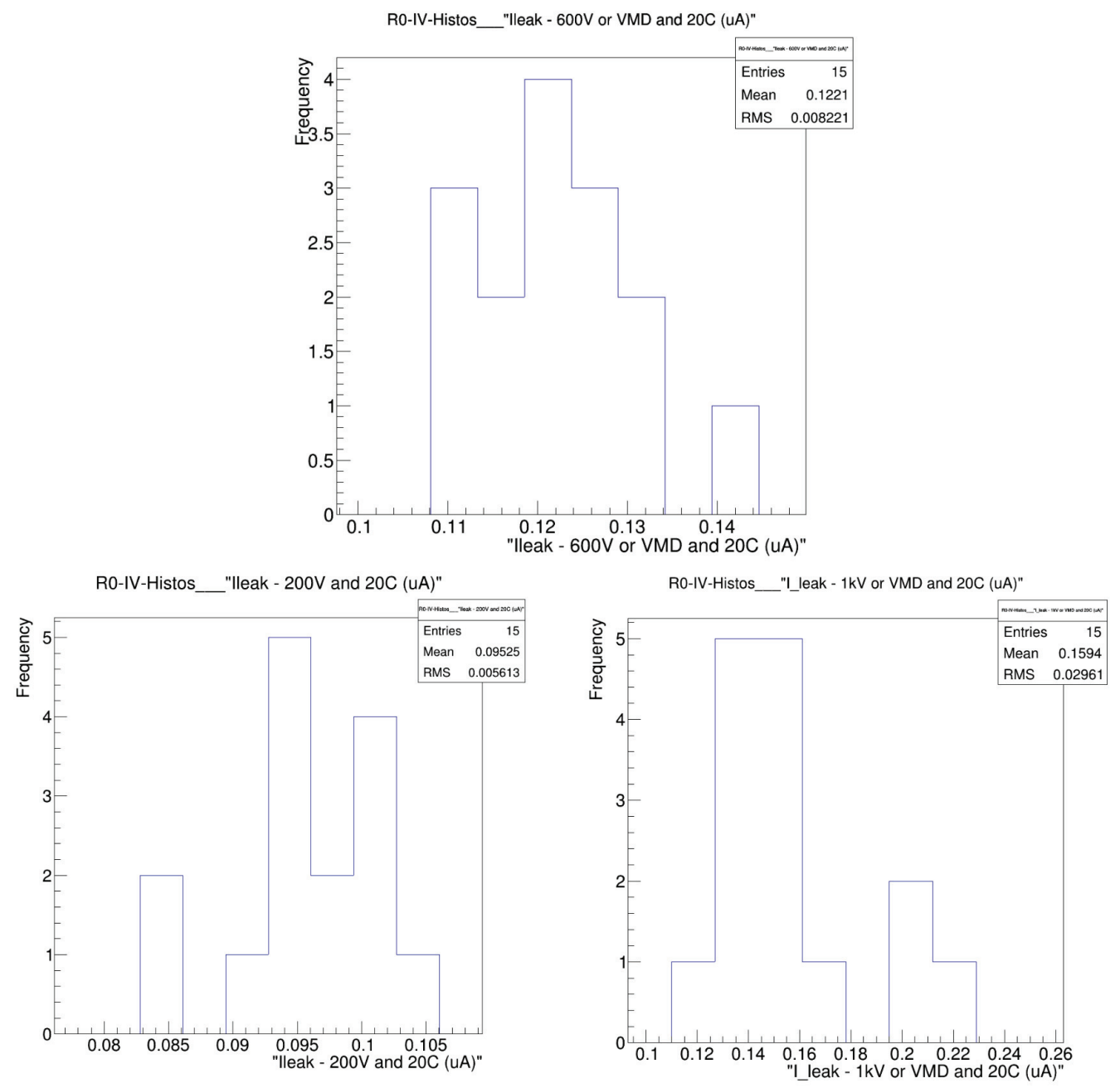

Figure 5.16: Histogrammed leakage currents results of the Carleton ATLA12EC data at various voltages during IV curve. Top; $600 \mathrm{~V}$ ITk operation proxy. Bottom; QA interesting voltages of $200 \mathrm{~V}$ (left) and $1000 \mathrm{~V}$ (right). 


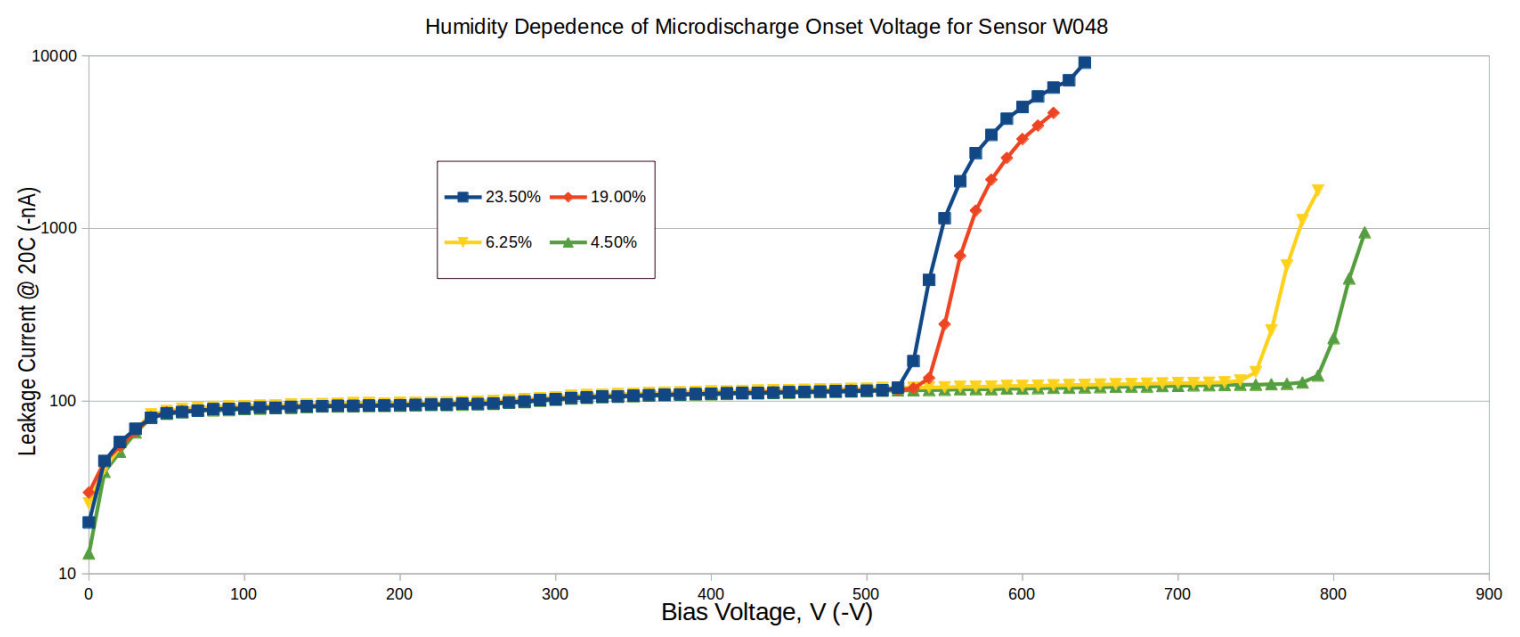

Figure 5.17: The inverse relation observed between humidity and microdischarge onset voltage is seen in this plot of sequential IV curve measurements at decreasing humidities. Carleton data for the ATLAS12EC sensor W048 is shown.

of this quantity is amplified.

As it is a test of the bulk silicon the experimental setup for the CV curve is very similar to that of the IV curve. For the majority of results the two tests are conducted subsequently with the only changes to the setup being circuitry changes affected at the patch panel. Thus tests have been conducted in the Karl Suss enclosure using both jig and probe needle contact, using the custom patch panels, and with extensive environmental monitoring.

The sensor bias is achieved with the same compliance limited SMU. For the AC measurement the state-of-the-art Wayne Kerr 6440B LCR meter with femto-farad resolution is used. The leads employed for the capacitance connections are coaxial (BNC) and the shields are connected to each other and the system ground as close to the sensor as possible using the smaller patch boxes (see Figure 5.11).

The inverse square relation of the bulk capacitance and the bias voltage, Equation 3.4 , is exploited for experimental determination of the full depletion depth. Conventional test parameters are; $10 \mathrm{~V}$ steps with 10 s settle time, a bias ramp from zero to $700 \mathrm{~V}$, and a 1 or $10 \mathrm{kHz}$ series resistance-capacitance measurement with $0.1 \mathrm{~V} \mathrm{AC}$ test voltage. Plots of $C^{-2}$ versus bias voltage are produced and show the marked two region structure of the resistor-like linear rise of the depleting sensor and the nearly flat plateau of the saturation (fully depleted) region. Linear fits to the two regions are conducted using least squares methodology. Degradation of the fits due to 
surface/edge effects (deviations from the linear character at the intersection) are minimized by requiring a coefficient of determination greater than 0.9 . This is achieved by sequential removal of the points closest to the intersection until the fits are well behaved.

The CV curve results for 15 ATLAS12EC prototypes obtained at Carleton are presented in the bottom plot of Figure 5.18. Results for 33 ATLAS12 and eight ATLAS07 are shown for comparison in the middle and top plots respectively. The Carleton results for the 15 ATLAS12EC are histogrammed in Figure 5.19 with an average full depletion voltage of $302.7 \mathrm{~V}$ and an RMS of $1.8 \mathrm{~V}$.

The full depletion voltages measured match the expectations of sensor resistivity and active depth for each submission $(200-250 \mathrm{~V}$ for the ITk-like value of the ATLAS07 and 300-320V for the lower quality silicon ATLAS12 and ATLAS12EC). The batch spread is seen to decrease over the iterations from roughly $22 \%$ (ATLAS07) to $2 \%$ (ATLAS12EC). More proof that manufacturing and sensor handling technique is being properly cultivated. Essentially all curves exhibit the smooth transition at the region of intersection that is indicative of surface effects. Likewise, the slight linear increase in the high bias saturation region that is indicative of over-depletion effects is also seen.

Sensors are compared to specifications on the resistivity and full active depletion depth which are obtained via Equations 3.1 and 3.4. The boundary cases for each of the resistivity and active depletion depth are considered. For the Carleton ATLAS12EC a resistivity of $3.4{\mathrm{k} \Omega \mathrm{cm}^{-2}}^{-}$is obtained for the largest possible active thickness and an active depth of $290 \mu \mathrm{m}$ (94\% of full thickness) for the lowest resistivity. Both cases match specification [30].

Equations 3.1 and 3.4 in Section 3.3 explain that the bulk capacitance during depletion is a function of the effective doping concentration in the depleted depth. Therefore, simple manipulation can yield the effective doping concentration as a function of bias voltage or even of depletion depth. This was conducted for the set of 15 ATLAS12EC sensors with the results shown in Figure 5.20. Several aspects of these curves can be interpreted as the effects of different surface features on the advance of the depletion front (from the isolated $n^{+}$strips, to encompass the strip structure, transitioning into a smooth front in the bulk). Interestingly, a small discrepancy in 

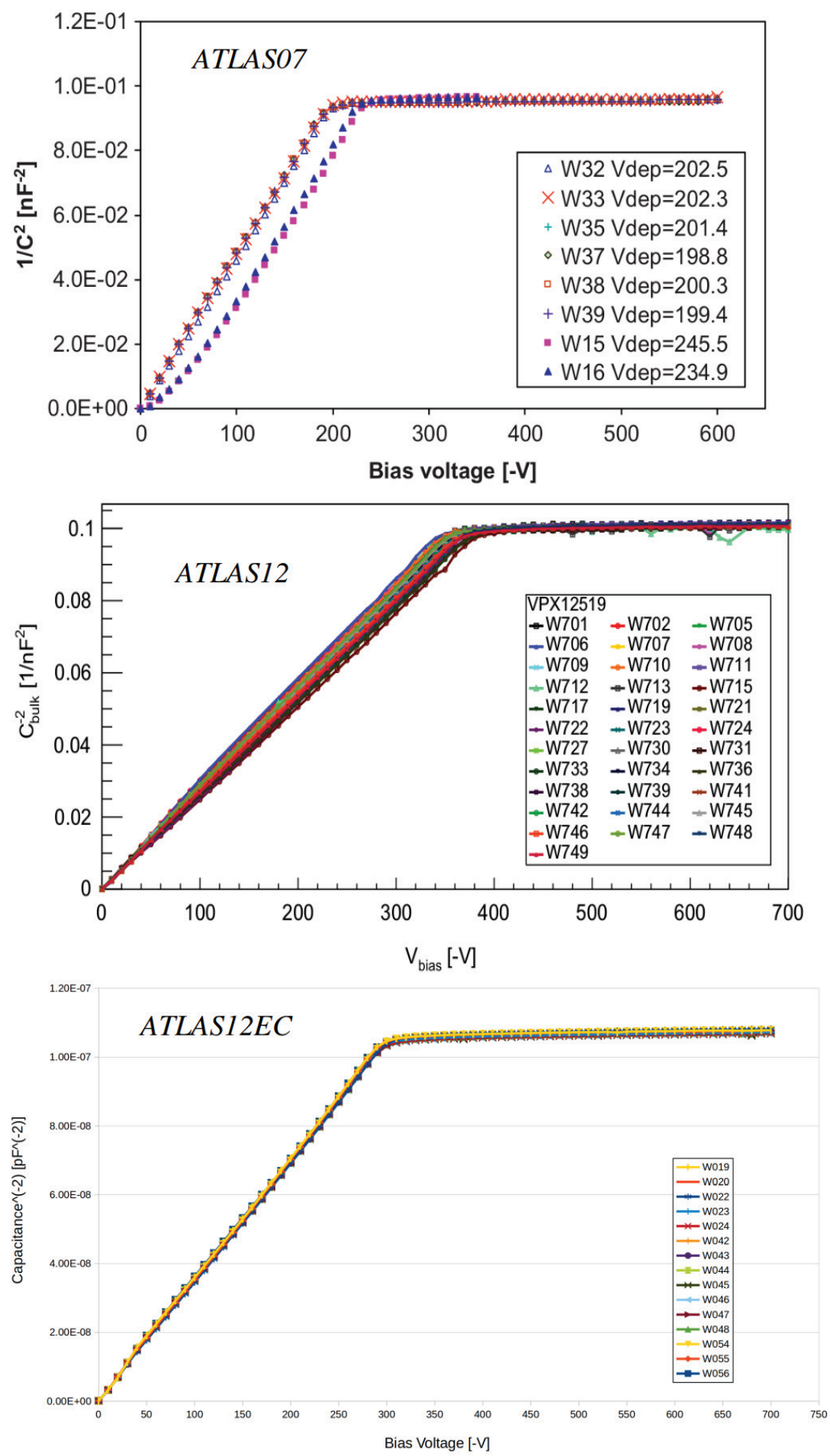

Figure 5.18: A comparison of CV curve results for the three most recent sensor submissions. Top plot; ATLAS07 with $V_{f d} \simeq 200-250 V$ [45]. Center plot; ATLAS12 with $V_{f d} \simeq 320 \mathrm{~V}[1,42]$. Bottom plot; ATLAS12EC with $V_{f d} \simeq 300 \mathrm{~V}$ (data obtained at Carleton). 


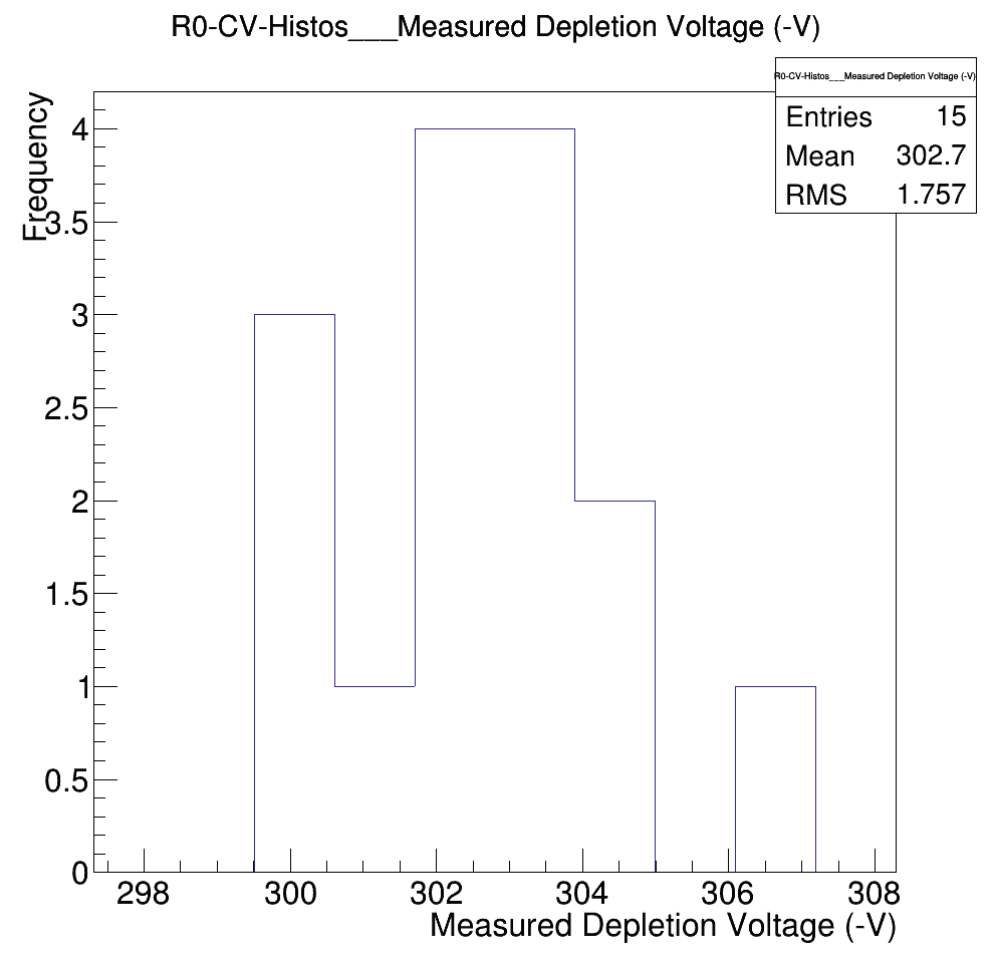

Figure 5.19: Histogrammed full depletion voltage results of the Carleton ATLAS12EC data. CV curve inference is used to obtain $V_{f d}$. An average full depletion voltage of $302.7 \mathrm{~V}$ and an RMS of $1.8 \mathrm{~V}$ is obtained for the 15 sensor sample. 


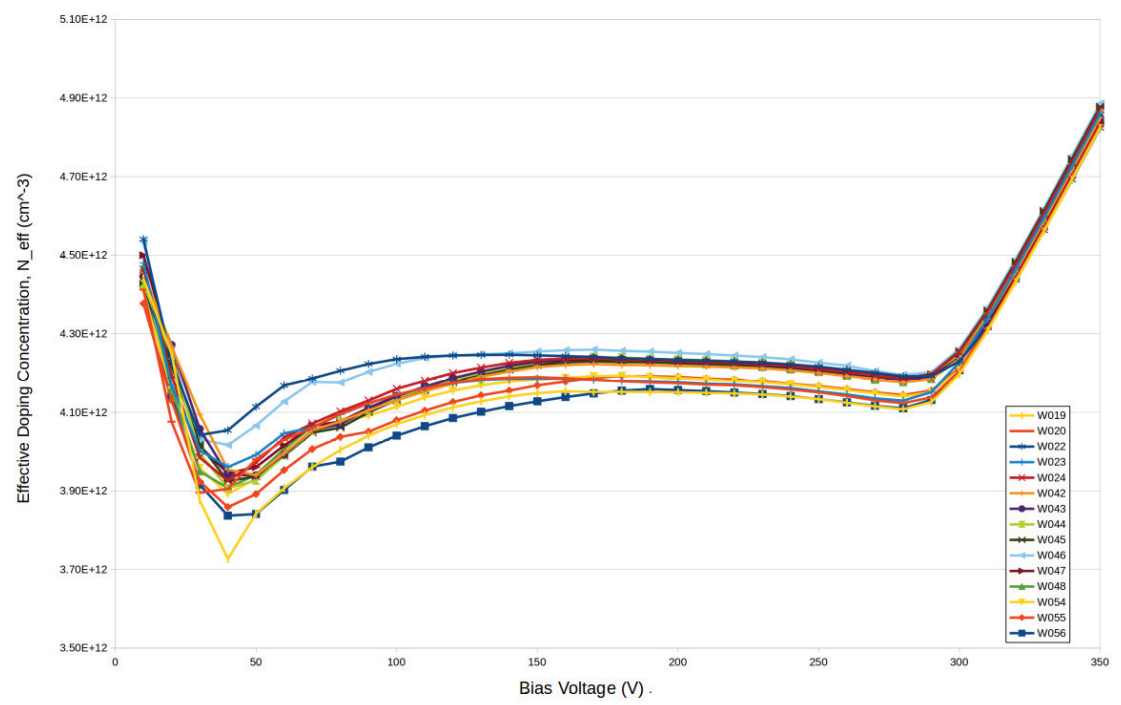

Figure 5.20: The effective doping concentration profile as a function of bias voltage for 15 ATLAS12EC sensors. A small batch-to-batch variation is seen in the steplike feature located in the range 60-80V. Data collected at Carleton University.

the feature profile of the curves was seen between subsets of the ATLAS12EC prototypes (in the slight decrease in doping concentration near 60-80V). This small effect was traced to the manufacturer batch (the Carleton set consists ofS sensors from two manufacturer batches) lending credence to the Carleton site's readiness for technical QA testing. Further investigation of the difference is forth-coming.

\subsubsection{Long Term IV Stability and its Humidity Dependency}

IV curves quickly illuminate any major issues in the sensor processing, to elucidate more minor issues endemic in the surface and edge long-term leakage current stability tests are conducted. The voltage at which the sensor is held at varies from sample to sample and test to test with usual choices of the maximal operating voltage $(700 \mathrm{~V})$ or the onset of microdischarge.

Conventional test parameters are used for the majority of tests; a data collection interval of 10s and a ramp to the test voltage with 10s long $10 \mathrm{~V}$ steps followed by a one minute settle after the ramp and before data collection begins. More variable is the test duration, voltage, humidity, and compliance setting. Long-term stability tests at Carleton have focused on investigating humidity dependency of the ATLAS12EC which is an active area of research in the collaboration. These tests and interpretation 
of their results are still underway with the current understanding summarized below.

Two of the 15 R0 prototype sensors were chosen for an investigation of the humidity, humidity history, and bias history dependencies of the IV character. One sensor, W042, performed well during the initial characterization (an average sensor) while the other, W048, was the only Carleton sensor to exhibit microdischarge. The inverse relation between humidity and microdischarge onset voltage observed during initial characterization for this latter sensor is shown in Figure 5.17. It can be noted that no special care was used in controlling the humidity during initial characterization (Figures 5.14, 5.15, 5.16, 5.18, and 5.19) except for those sequential tests of W048.

Combined data (leakage current, temperature, and humidity) plots of tests conducted at Carleton are shown in Figure 5.21. The top and centre plots show multiple hour test results for the humidity sensitive W048 sensor. Data at $25 \pm 5 \% R H$ and $36 \pm 5 \% R H$ (respectively) is shown. The onset voltage of microdischarge is used for bias in these stability measurements, and as is therefore variable $(280 \mathrm{~V}$ and $450 \mathrm{~V}$ respectively). The bottom plot in Figure 5.21 shows the average sensor, W042, at the maximal operating voltage and a humidity of $36 \pm 2 \%$.

Comparison of the bottom two plots shows the increased humidity sensitivity of W048, indications that the surface or edge processing of this sensor is sub-par. It can be noted that no temperature correction is applied in these results causing the un-horizontal leakage current baselines observed in the left and right plots.

Tests with excursions to higher humidities were also conducted with observation of strong variations in the leakage current of both sensors (variations began at $\sim 40 \%$ for W048, and 50\% for W042). In some tests, rises and falls in the current were directly correlated with rises and falls in the humidity.

A natural result of the testing procedure was the use of bias voltages slightly below that of microdischarge onset. This variability resulted in the observed hysteresis shown in Figure 5.22 (refer Section 3.3.6). Although the result is confused by the unsystematic approach to this effect during the humidity tests the general trend of increasing hysteresis with longer, wetter, preceding voltage applications and less preceding dry storage time is seen. Both sensors exhibited hysteresis with the humidity sensitive W048 shown in the figure.

These results lead to the conclusion that the mobile ion effect is non-neglible in the ITk Strip sensors. As seen in Section 3.3.6, it is a surface phenomena related 

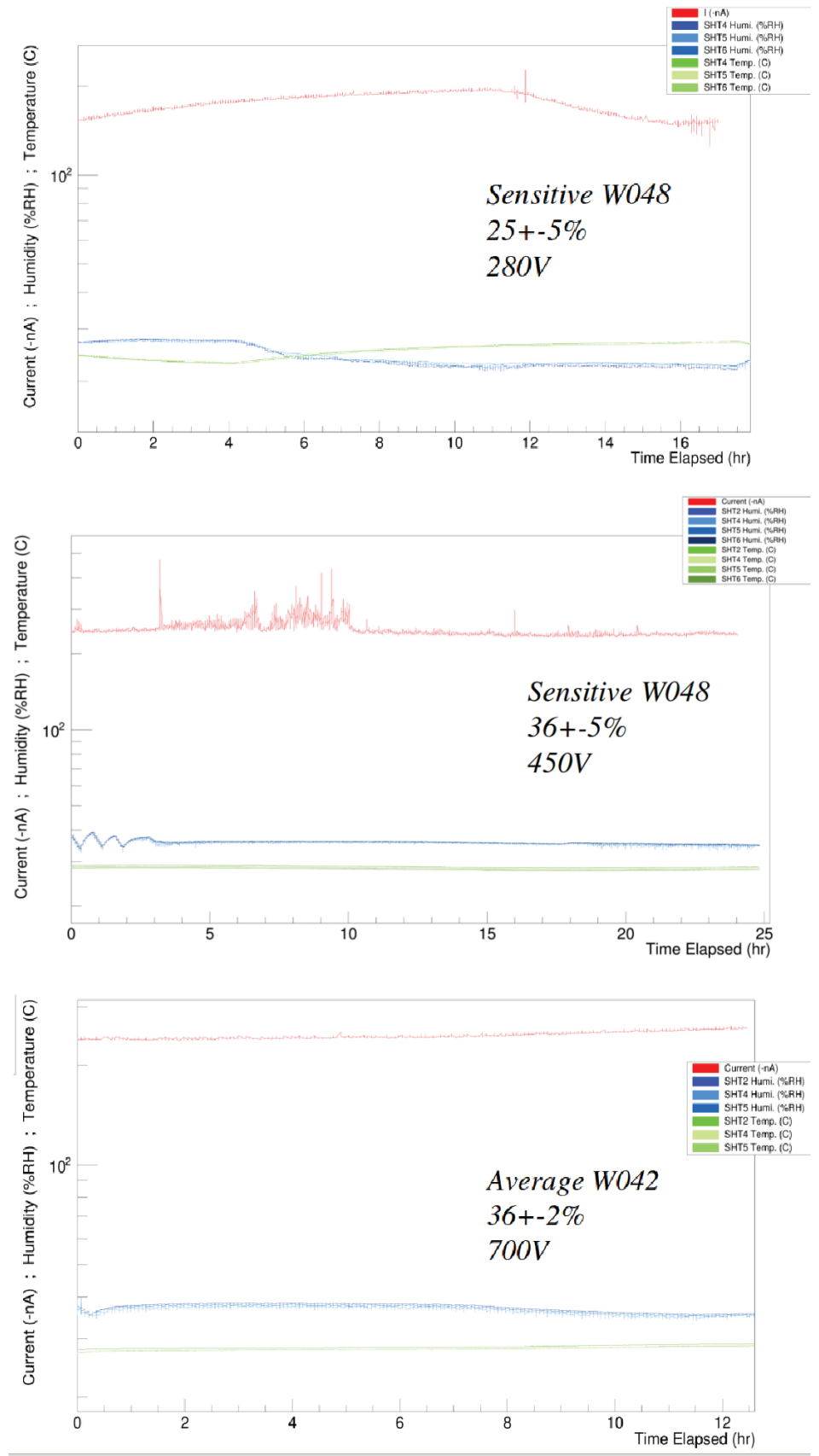

Figure 5.21: Combined data results for leakage current long-term stability tests of two ATLAS12EC sensors. Red is leakage current in $\mu A$, green is temperature in ${ }^{\circ} \mathrm{C}$, and blue is humidity in $\% R H$. Top; the humidity sensitive W048 at $25 \pm 5 \% R H$ for $18 \mathrm{hr}$. Centre; the humidity sensitive W048 at $36 \pm 5 \% R H$ for $24 \mathrm{hr}$. Bottom; the average (insensitive) W042 sensor at $36 \pm 2 \% R H$ for $12 h r$. Temperature corrections are not applied and a temporal offset exists in the cyclic environmental monitoring of the first plot (trends in reality line up). Data collected at Carleton University. 


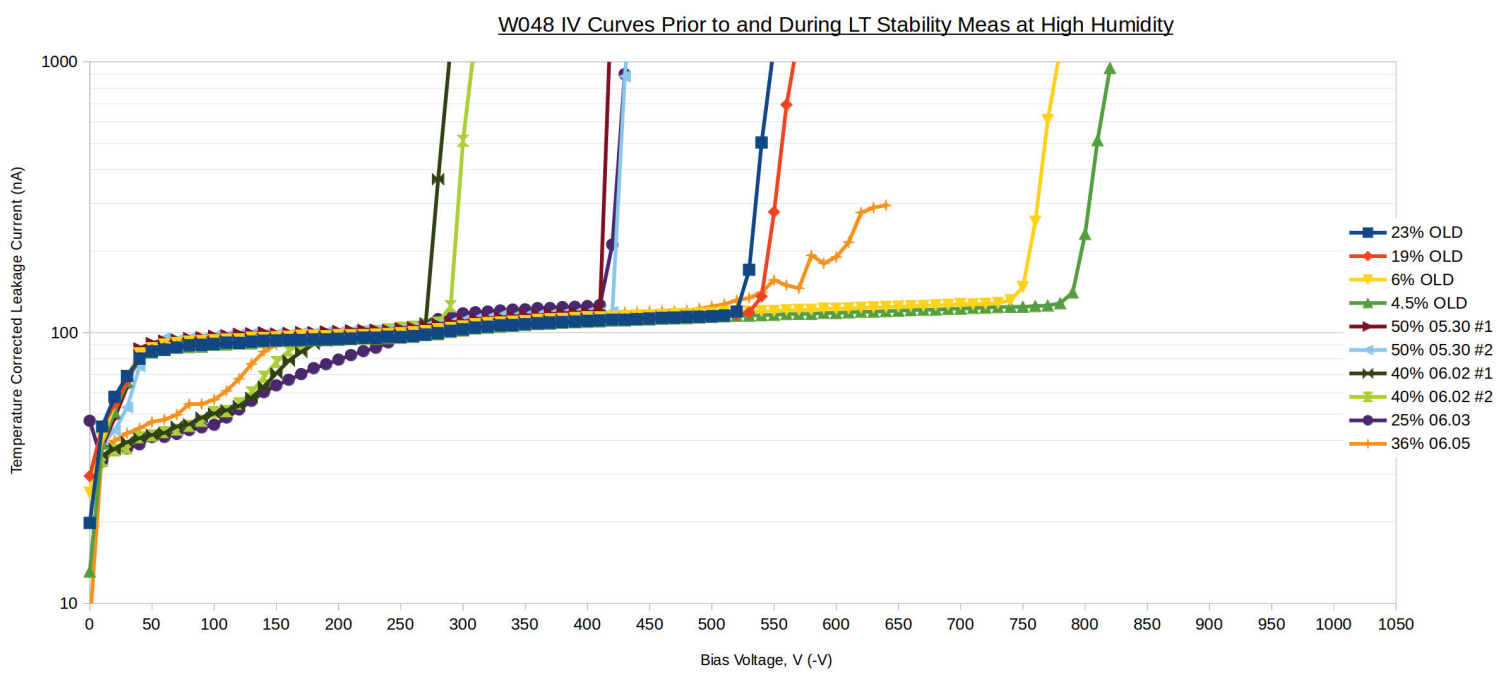

Figure 5.22: Hysteresis in an ATLAS12EC sensor (the humidity sensitive W048). OLD refers to April of the same year. Dry storage conditions between tests were variable but recorded. Data collected at Carleton University.

to high electric field regions in the MOS structure of the aluminum. The question that needs to be asked is whether these regions are predominantly associated with contamination or with design. The answer in either respect is extremely important for the eventual full-scale production run of $\sim 18000$ sensors.

An interesting result that is perhaps applicable is given in Figure 4.25. There the IV character is seen to change with the slim edge dicing in a manner that is related to the strip geometry on the sensor. The late rise in leakage current (from atop the second plateau) seen for the stereo strip implementing A12M sensors is also seen in some of the ATLAS12EC results (Figure 5.15). This points the finger towards the sensor layout. However, the very recent nature of the presented results and the lack of long-term testing literature results for ITk prototypes mean that the true answer to this question is yet uncovered. This analysis serves as the next step for the now established Carleton sensor testing and R\&D site. 


\section{Chapter 6}

\section{Summary and Conclusions}

This document has outlined the importance of my work as a member of the ITk Strip collaboration in the greater scale of the HL-LHC upgrade. The main motivations for the detector development and evaluation are the instantaneous and integrated luminosity targets of this next-generation collider. These have necessitated the vast number of innovations that are implemented at every level in the accelerator complex (from the machine itself down to the ITk Strip sensors). These technological advancements place the ITk and HL-LHC at the forefront of experimental high energy physics. A position they will hold until 2037 at the earliest.

The current-generation LHC has been in this position for seven years now and dictates a great deal of our theoretical understanding of the universe. The general statistical enhancement offered by the high luminosity upgrade has the effect of reducing uncertainty in any physics analyses within the $14 T e V$ energy capability. It is one of humanity's best chances at discovering new physics. The ITk is the most important upgrade for the general purpose ATLAS detector at the HL-LHC. One that is required if ATLAS is to hope to survive the new grueling conditions.

My contributions discussed in this paper focus on the two aspects of showing that the ITk performance requirements will be met and fundamental preparations for the Strip detector production.

The larger of the two in a workload respect was the latter. My goal in this case was the construction of an endcap sensor testing site capable of verification of one quarter of the production Strip sensors $(\sim 5000)$. Since these devices will be placed in the ITk for ten years of minimal-intervention operation an extreme level of reliability and experience is necessary in the integral quality assessment. I have performed the majority of the activation-energy-barrier overcoming work, bringing 
the Carleton University site from an essentially non-existent entity to the point of publishable results (as given in the last section).

The amount of work required to build the infrastructure is not given to scale in the document text. It was a long and arduous progress which required the effective synergy of many diverse fields; physics, chemistry, electronics, and computer programming are examples. Missing infrastructure which will arrive by the end of the year is essentially all that is left for full production capability at the site.

Experience with the ITk Strip sensors at Carleton University was minimal prior to my project's start. Accumulation of knowledge is thus a significant contribution to this part of my work. In this aspect one could ask the question of the reader; do you think you are ready to test an ATLAS12EC sensor? The only missing pieces, I hope, are the technical operational manuals that derive from the knowledge here and the experience of conducting the tests yourself.

The test results for the novel, large area, radiation hard ATLAS12EC sensors, shown in Sections 5.3 and 5.5 are the proof of the Carleton University sensor testing capability. As well, they chronicle an extremely important step in the ITk Strip sensor design validation.

The ATLAS12EC are the first stereo annulus sensors ever fabricated. This promising sensor shape is planned for use throughout the ITk Strip endcaps and these tests are absolutely fundamental to judge its suitability. It is expected that this (and follow-up) legacy-continuing work will be published in a series of papers similar to those produced for the ATLAS07 [26,29,39,45] and ATLAS12 [10,24,25,42,83] sensor submissions. The first of these are expected by the end of this year.

Aside from my sensor-related work, I also briefly discussed my involvement in the very important 2016 ITk Strip testbeam campaign. The text is again woefully misleading in regards to the amount of work completed (an approach that was taken deliberately to avoid a large explosion in page count). Discussion is limited to the end-of-lifetime signal to noise ratio "money plot", Figure 2.14. This figure shows that the ITk (Strip) will meet the HL-LHC needs and, therefore, that the high luminosity physics gains are achievable. It is the first such measurement using the $130 \mathrm{~nm}$ based Strip detector electronics and HL-LHC fluences. I was intimately involved in the setup and measurements that produced this fundamental result. 


\section{List of References}

[1] T. A. Collaboration, "Atlas inner tracker strip detector: Technical design report," Tech. Rep. ATL-TDR-025 / LHCC-2017-005, 2017.

[2] T. A. Collaboration, "Atlas inner detector: Technical design report vol 1.," Tech. Rep. ATL-TDR-4 / CERN-LHCC-97-16, 1997.

[3] C. H.-L. Collaboration, "The hl-lhc project public webpages." http:// hilumilhc.web.cern.ch/.

[4] Y. Unno, "Development of radiation-tolerant silicon microstrip sensor for the atlas inner tracker at the slhc," Nuclear Instruments and Methods in Physics Research A, vol. 623, pp. 165-167, 2010.

[5] CERN, "Cern public webpages." cern.home.

[6] C. L. H. C. S. W. Group, "Handbook of lhc higgs cross sections: 3. higgs properties : Report of the lhc higgs cross section working group," Tech. Rep. CERN2013-004, 2013.

[7] P. Vankov, "Development of large area silicon detectors," Tech. Rep. ATLUPGRADE-PROC-2012-003, CERN, 2012. arXiv:1201.5469.

[8] ATLAS, "Atlas public webpages." atlas.cern.

[9] K. Potamianos, "The upgraded pixel detector and the commissioning of the inner detector tracking of the atlas experiment for run-2 at the large hadron collider," ATLAS Conf. Proc. ATL-PHYS-PUB-2014-016, 2014.

[10] Y. U. et al., "Development of $\mathrm{n}+$ - in - p large-area silicon microstrip sensors for very high radiation environments - atlas12 design and initial results," Nuclear Instruments and Methods in Physics Research A, vol. 765, pp. 80-90, 2014.

[11] M. Peskin and D. Schroeder, An Introduction to Quantum Field Theory. Addison-Wesley Publishing Company, 1995.

[12] T. A. Collaboration, "Projections for measurements of higgs boson signal strengths and coupling parameters with the atlas detector at the hl-lhc," ATLAS Note. ATL-PHYS-PUB-2014-016, 2014.

[13] T. A. Collaboration, "Projections for measurements of higgs boson cross sections, branching ratios and coupling parameters with the atlas detector at a hl-lhc," ATLAS Note. ATL-PHYS-PUB-2013-014, 2013. 
[14] I.-M. Gregor, "The itk strip project," 2014.

[15] C. Sawyer, "Strips architecture," 2017.

[16] C. Kittel, Introduction to Solid State Physics. New York: John Wiley and Sons, 2005.

[17] S. Sze, Physics of Semiconductor Devices. New York: John Wiley and Sons, 1969.

[18] T. H. F. Group, "Silicon detectors gif."

[19] L. P. et al., "Observations for irradiated sensors from desy and diamond testbeam," 2017.

[20] Y. T. et al., "Structure of the silicon-oxide interface," Thin Solid Films, vol. 400, pp. 95-100, 2001.

[21] T. O. et al., "Microdishcharges of ac-coupled silicon strip sensors," Nuclear Instruments and Methods in Physics Research A, vol. 342, pp. 22-26, 1994.

[22] Y. N. et al., "Anomolous iv behaviour of atlas sct microstrip sensors," Nuclear Instruments and Methods in Physics Research A, vol. 579, pp. 812-816, 2007.

[23] I. P. et al., "Radiation-induced point- and cluster-related defects with strong impact on damage properties of silicon detectors," Nuclear Instruments and Methods in Physics Research A, vol. 611, pp. 52-68, 2009.

[24] M. M. et al., "Study of surface properties of atlas12 strip sensors and their radiation resistance," Nuclear Instruments and Methods in Physics Research A, vol. 831, pp. 197-206, 2016.

[25] K. H. et al., "Charge collection and field profile studies of heavily irradiated strip sensors for the atlas inner tracker upgrade," Nuclear Instruments and Methods in Physics Research A, vol. 831, pp. 1-8, 2016.

[26] K. H. et al., "Testing of bulk radiation damage of n-in-p silicon sensors for very high radiation enviroments," Nuclear Instruments and Methods in Physics Research A, vol. 636, pp. S83-S89, 2011.

[27] M. B. et al., "Radiation-hard semiconductor detectors for superlhc," Nuclear Instruments and Methods in Physics Research A, vol. 541, pp. 189-201, 2005.

[28] Y. U. et al., "p-bulk silicon microstrip sensors and irradiation," Nuclear Instruments and Methods in Physics Research A, vol. 579, pp. 614-622, 2007.

[29] Y. U. et al., "Development of n-on-p silicon sensors for very high radiation environments," Nuclear Instruments and Methods in Physics Research A, vol. 636, pp. S24-S30, 2011.

[30] T. A. U. S. S. Collaboration, "Supply of silicon microstrip sensors of atlas12ec specification," Technical Specification, CERN, 2015. 
[31] T. A. I. S. S. Collaboration, "Technical specification for the supply of atlas17ls strip sensors," Tech. Rep. DO-30717/EP/ATLAS, CERN, 2017.

[32] V. B. et al., "Sensors for the end-cap prototype of the inner tracker in the atlas detector upgrade," Nuclear Instruments and Methods in Physics Research A, vol. 833, pp. 226-232, 2016.

[33] G. C. et al., "First results on charge collection efficiency of heavily irradiated microstrip sensors fabricated on oxygenated p-type silicon," Nuclear Instruments and Methods in Physics Research A, vol. 518, pp. 340-342, 2004.

[34] G. C. et al., "Improving the radiation hardness properties of silicon detectors using oxygenated n-type and p-type silicon," Nuclear Instruments and Methods in Physics Research A, vol. 47 3, pp. 527-532, 2000.

[35] G. C. et al., "First results on the charge collection properties of segmented detectors made with p-type bulk silicon," Nuclear Instruments and Methods in Physics Research A, vol. 487, pp. 465-470, 2002.

[36] G. C. et al., "Effects of the accelerated annealing on p-type silicon micro-strip detectors after very high doses of proton irradiation," Nuclear Instruments and Methods in Physics Research A, vol. 568, pp. 46-50, 2006.

[37] T. R. Collaboration, "Rd50 status report 2009/2010 - radiation hard semiconductor devices for very high luminosity colliders," Tech. Rep. CERN-LHCC-2012010 / LHCC-SR-004, CERN, 2012.

[38] Y. U. et al., "Evaluation of test structures for the novel $\mathrm{n}+$ - in - p pixel strip sensors for very high radiation environments," Nuclear Instruments and Methods in Physics Research A, vol. 731, pp. 183-188, 2013.

[39] S. L. et al., "Testing of surface properties pre-rad and post-rad of n-in-p silicon sensors for very high radiation environment," Nuclear Instruments and Methods in Physics Research A, vol. 636, pp. S111-S117, 2011.

[40] S. M. et al., "Evaluation of slim-edge, multi-guard, and punch-through-protection structures before and after proton irradiation," Nuclear Instruments and Methods in Physics Research A, vol. 699, pp. 36-40, 2013.

[41] Y. U. et al., "Development of n-in-p silicon planar pixel sensors and flip-chip modules for very high radiation environments," Nuclear Instruments and Methods in Physics Research A, vol. 650, pp. 129-135, 2011.

[42] L. H. et al., "Detailed studies of full-size atlas12 sensors," Nuclear Instruments and Methods in Physics Research A, vol. 831, pp. 167-173, 2016.

[43] M. U. et al., "Embedded pitch adapters: A high-yield interconnection solution for strip sensors," Nuclear Instruments and Methods in Physics Research A, vol. 831, pp. 221-228, 2016.

[44] Y. U. et al., "Slhc upgrade of the atlas sct tracker," Nuclear Instruments and Methods in Physics Research A, vol. 612, pp. 439-447, 2010. 
[45] J. B. et al., "Evaluation of the bulk and strip characteristics of lrage area nin-p silicon sensors intended for a very high radiation environment," Nuclear Instruments and Methods in Physics Research A, vol. 636, pp. S104-S110, 2011.

[46] M. Krammer, "The update of the european strategy for particle physics," Physica Scripta, vol. T158 014019, pp. 1-7, 2013.

[47] CERN, "2014 yellow report," Tech. Rep. CERN-2014-004, pp. 177-181, 2014. arXiv:1410.5646.

[48] W. Leo, Techniques for Nuclear and Particle Physics Experiments: A How-to Approach. Springer-verlag-Berlin Heidleberg, 1987.

[49] G. Knoll, Radiation Detection and Measurements. New York: John Wiley and Sons, 2000.

[50] L. Hommels, "personal correspondence with l.b.a. hommels."

[51] K. S. et al., "Charge collection efficency in a semiconductor radiation detector with a non-constant electric field," Nuclear Instruments and Methods in Physics Research A, vol. 37 2, pp. 183-185, 1990.

[52] A. Chilingarov, "Temperature dependence of the current generated in the si bulk," Journal of Instrumentation, vol. 8 10, p. P10003, 2013.

[53] T. O. et al., "Micro-discharge noise and radiation damage of silicon microstrip sensors," Nuclear Instruments and Methods in Physics Research A, vol. 383, pp. 166-173, 1996.

[54] Y. U. et al., "Optimization of surface structures in n-in-p silicon sensors using tcad simulation," Nuclear Instruments and Methods in Physics Research A, vol. 636, pp. S118-S124, 2011.

[55] T. K. et al., "Systematic study of micro-discharge characteristics of atlas barrel silicon microstrip modules," Nuclear Instruments and Methods in Physics Research A, vol. 579, pp. 782-787, 2007.

[56] T. P. et al., "Charge losses in segmented silicon sensors at the si-sio2 interface," Nuclear Instruments and Methods in Physics Research A, vol. 700, pp. 22-29, 2013.

[57] J. S. et al., "Surface effects in segmented silicon sensors," Nuclear Instruments and Methods in Physics Research A, vol. 845, pp. 159-163, 2017.

[58] A. L. et al., "Instability of the behaviour of high resistivity silicon detectors due to the presence of oxide charges," Nuclear Instruments and Methods in Physics Research A, vol. 288, pp. 35-43, 1990.

[59] G.Casse, "Prediction of the performances of finely segmented si detector for tracking applications in the future super-colliders after severe radiation damage," Nuclear Instruments and Methods in Physics Research A, vol. 566, pp. 26-34, 2006. 
[60] M. M. et al., "Study of anomalous charge collection efficiency in heavily irradiated silicon strip detectors," Nuclear Instruments and Methods in Physics Research A, vol. 636, pp. S50-S55, 2011.

[61] J. M. et al., "The effect of radiation induced defects on the performance of high resistivity silicon diodes," Nuclear Instruments and Methods in Physics Research A, vol. 377, pp. 224-227, 1996.

[62] A. C. et al., "Radiation studies and operational projections for silicon in the atlas inner detector," Nuclear Instruments and Methods in Physics Research A, vol. 360, pp. 432-437, 1995.

[63] G. B. et al., "Temperature dependence of reverse annealing in bulk damaged silicon," Nuclear Physics B (Proc. Suppl.), vol. 44, pp. 524-527, 1995.

[64] A. A. et al., "The silicon microstrip sensors of the atlas semiconductor tracker," Nuclear Instruments and Methods in Physics Research A, vol. 578, pp. 98-118, 2007.

[65] G. C. et al., "Introduction of high oxygen concentrations into silicon wafers by high-temperature diffusion," Nuclear Instruments and Methods in Physics Research A, vol. 438, pp. 429-432, 1999.

[66] G. K. et al., "Investigations on the passivation mechanism of aln:h and aln:h-sin:h stacks," Energy Procedia, vol. 55, pp. 797-804, 2014.

[67] T. A. U. S. S. Collaboration, "Supply of silicon microstrip sensors of atlas12 specification," Technical Specification, CERN, 2012.

[68] D. R. et al., "Silicon microstrip detectors for the atlas sct," Nuclear Instruments and Methods in Physics Research A, vol. 485, pp. 84-88, 2002.

[69] E. F. et al., "Development of large area silicon detectors," Nuclear Instruments and Methods in Physics Research A, vol. 253, pp. 467-477, 1987.

[70] T. R. Collaboration, "Progress report on the rd2 project," Tech. Rep. CERN/DRDC 94-34 / RD-2 Status Report, CERN, 1994.

[71] G. T. et al., "Radiation induced bulk damage in silicon detectors - rd2 collaboration," Nuclear Instruments and Methods in Physics Research A, vol. 383, pp. 144-154, 1996.

[72] C. R. Collaboration, "The rose collaboration - rd48." http://rd48.web.cern. $\mathrm{ch} / \mathrm{RD} 48 /$.

[73] B. D. et al., "Experimental results on radiation induced bulk damage effects in float-zone and epitaxial silicon detectors," Nuclear Instruments and Methods in Physics Research A, vol. 386, pp. 162-166, 1997.

[74] C. R. Collaboration, "Rd50 - radiation hard semiconductor devices for very high luminosity colliders." https://rd50.web.cern.ch/rd50/. 
[75] G. C. et al., "Comparison of charge collection efficency of segmented silicon detectors made with $\mathrm{fz}$ and mcz p-type silicon substrates," Nuclear Instruments and Methods in Physics Research A, vol. 591, pp. 178-180, 2008.

[76] S. T. et al., "Proton irradiation on p-bulk silicon strip detectors using 12 gev ps at kek," Nuclear Instruments and Methods in Physics Research A, vol. 383, pp. 159-165, 1996.

[77] K. H. et al., "Performance of irradiated n+ - on - p silicon microstrip sensors," Nuclear Instruments and Methods in Physics Research A, vol. 565, pp. 538-542, 2006 .

[78] Y. U. et al., "Novel p-stop structure in n-side of silicon microstrip detector," Nuclear Instruments and Methods in Physics Research A, vol. 541, pp. 40-46, 2005

[79] T. A. et al., "Beam study of irradiated atlas-sct prototypes," Nuclear Instruments and Methods in Physics Research A, vol. 485, pp. 67-72, 2002.

[80] F. A. et al., "Beam test of the atlas silicon detector modules," Nuclear Instruments and Methods in Physics Research A, vol. 409, pp. 236-239, 1998.

[81] T. A. S. Collaboration, "Supply of silicon microstrip sensors of atlas07 specification," Technical Specification, CERN, 2007.

[82] T. K. et al., "Beam test of novel n-in-p strip sensors for high radiation environment," Nuclear Instruments and Methods in Physics Research A, vol. 731, pp. 247-251, 2013.

[83] R. M. et al., "Evalution of the performancce of irriadiated silicon strip sensors for the forward detector of the atlas inner tracker upgrade," Nuclear Instruments and Methods in Physics Research A, vol. 831, pp. 207-212, 2016.

[84] M. A. et al., "A forward silicon strip system for the atlas hl-lhc upgrade," Nuclear Instruments and Methods in Physics Research A, vol. 730, pp. 210-214, 2013.

[85] R. D. et al., "Technology computer-aided-design home page - stanford university." http://www-tcad.stanford.edu/.

[86] R. M.-H. et al., "A portable readout system for microstrip silicon sensors (alibava," IEEE Transactions On Nuclear Science, vol. 56 3, pp. 1642-1649, 2009. 
Appendix A

Abbreviations Glossary 
ABC ATLAS Binary Chip

AIDA Advanced European Infrastructures for Detectors at Accelerators

AMAC Autonomous Monitor and Control chip

ASIC Application Specific Integrated Circuit

ATLAS A Toroidal LHC Apparatus

b $\quad$ barn $\left(1 b=10^{-28} m^{2}\right)$

BNL Brookhaven National Lab

CCE Charge Collection Efficiency

CERN Conseil Europen pour la Recherche Nuclaire

CMOS Complimentary MOS

CMS Compact Muon Solenoid

CVD Chemical Vapour Deposition

CYRIC Cyclotron and Radioisotope Center

$\mathrm{Cz} \quad$ Czochralski (silicon)

DCS Data Control Systems

DESY Deutsches Elektronen-Synchrotron

DLTS Deep Level Transient Spectroscopy

DOFZ Diffusion Oxygenated Float-Zone (silicon)

DUT Device Under Test

ENC Equivalent Noise Charge

EoS End of Structure

EWSB Electroweak Symmetry Breaking 
FE Front-End

FOXFET Field-Oxide Field-Effect Transistor

FZ Float-zone (silicon)

GPIB General Purpose Interface Bus

GR Guard Ring

HCC Hybrid Control Chip

HL-LHC High Luminosity - LHC

ID Inner Detector

ISO International Standards Organization

ITk Inner Tracker

KIT Karlsruhe Institute of Technology

LEP Large Electron Positron Collider

LHC Large Hadron Collider

lpGBTx low power Giga-Bit Transceiver

LS (pseudo-)Long Strip (test module)

LSM Laser Scanning Microscope

LVDS Low Voltage Differential Signal

$\mathrm{MCz} \quad$ Magnetic Czochralski (silicon)

mip minimum ionizing particle

MOS Metal-Oxide-Semiconductor

MOSFET Metal-Oxide Field-Effect Transistor

$n_{\text {eqv }} \quad 1 \mathrm{MeV}$ NIEL neutron equivalents 
NIEL Non-Ionizing Energy Loss

PCB Printed Circuit Board

PDE Partial Differential Equation

Pixel ITk Pixel Detector

PSI Paul Scherrer Institute

PTP Punch Through Protection

QA Quality Analysis

QC Quality Control

R\&D Research and Development

R0 Ring-0

SCLA Space Charge Limited Avalanche

SCPI Standard Commands for Programmable Instruments

SCT ATLAS (microstrip) SemiConductor Detector

SEU Single Event Upset

SMU Source-Monitor Unit

SPS Super Proton Synchrotron

Strip ITk Strip Detector

TDR Technical Design Report

TID Total Ionizing Dose

TRIGA Training, Research, Isotopes, General Atomics

TRT Transition Radiation Tracker

TSC Thermally Stimulated Current

TTC Timing, Trigger, and Control

VTRx+ Versatile link fibre optics driver 